\title{
Short-term Local Forecasting by Artificial Intelligence Techniques and Assess Related Social Effects from Heterogeneous Data
}

\author{
by \\ Bing Gong \\ Submitted to the ETSII \\ in partial fulfillment of the requirements for the degree of \\ Doctor of Philosophy in DEGIN program
}

at the

Universidad Politécnica de Madrid

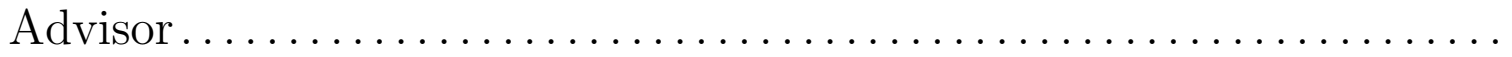

Joaquín Ordieres-Meré

Full Professor

Thesis Supervisor 


\section{Acknowledgments}

In the last four years, I have been dedicated myself to a long journey - pursuing a Ph.D. degree. This journey has been full of both painful and enjoyable experiences for me no matter in the academic world or the daily living life. I have been feeling so lucky and grateful that I have many supports, encouragements, and help from my advisors, my family, and friends. Without them, I could not complete my Ph.D. degree.

First and foremost, I am greatly indebted to my Ph.D advisor - Professor Joaquín Ordieres-Meré. I would like to give my sincere thanks to him for his advices, supports, and patience in guiding and helping me in any way he could. I had been so confused about my research at beginning of this Ph.D. and have encountered so many difficulties in doing research. He helps me to solve the programming issues every time I ask help from him. He modified my proposal, papers, and thesis so carefully. His wealth of knowledge, his intelligence and his way of hard working in particular inspiring. His advice can always guide me in the right direction. I have learned a lot from him. Without his advice and help, I could not finish my thesis successfully.

I would like to give my special thanks to my parents for their understanding and selfless loving. No matter what decision I made, they fully support me. I have been felt so sorry that I have been stayed so far away from them and I could not accompany nor take care of them. But I hope that they can be proud of me, which is one of my great motivation to work hard now and in the future.

Thanks to my supervisor Srinivas Talluri for offering such an opportunity to visit Michigan State University. It was been a wonderful experience working with you and knowing people there. Thank you for guiding me the research, which has consisted of an important part of my thesis.

I am indebted to my following friends: Zheng Xiao Chen, Sun Sheng Jing, Li Ning, Qubo. They are like my family members. My best moments in Spain are being with all of you. We always encourage each other when we met difficulties. This is one of the most cherished friendships in my life. Sheng Jing, thank you so much 
for being the one that accompanies and takes care of me when I need help. Also, I am greatly appreciated Joyce Wang and Yu Fei that offered me facilities during my stay in the United States. Thanks to my lovely friend, Zhang Jia Yi. We grew up together. Thank you for spending time with me, talking to me, and encouraging me no matter whenever I need you. Also, I appreciate my English teacher Tom, my flatmates Madelyn and Joo. They helped me to correct grammar mistakes for my paper and thesis, making me improve my English speaking and writing.

My thanks are given to my universities: China University of Geosciences (Wuhan) and Technical University of Madrid for offering me the best environment to study and do research. Thanks to the technical support from Centro de Supercomputación y Visualización de Madrid (CESVIMA) at Technical University of Madrid. Because this $\mathrm{Ph}$.D. research is based on intensive computation, without their support, it is impossible to finish my studies on time. Also, thanks to the China scholarship Council(No. 201206410060) for the financial supports making all my Ph.D. research possible.

Last but not least, I would like to give my appreciate to all the reviewers that spend your valuable time on reading my thesis. 


\section{Contents}

$\begin{array}{lll}1 & \text { Introduction } & 17\end{array}$

1.1 Background $\ldots \ldots \ldots \ldots \ldots \ldots$

1.1 .1 Environment . . . . . . . . . . . . . . . . . . . . . . . 18

1.1 .2 Public health evaluation . . . . . . . . . . . . . . . . . . 19

1.1 .3 Energy . . . . . . . . . . . . . . . . . . . . . . . 21

1.2 Social-economic effects on pollution $\ldots \ldots \ldots \ldots \ldots \ldots$

1.3 Local Prediction. . . . . . . . . . . . . . . . . . . . . . 25

1.4 Air quality monitoring network reconfiguration . . . . . . . . . 27

1.5 Energy used awareness $\ldots \ldots \ldots$

1.6 Driven forces of dynamic changes: energy consumption, $\mathrm{CO}_{2}$ emission,

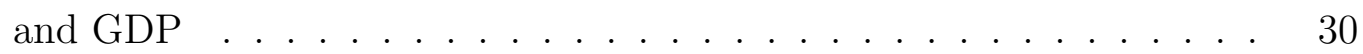

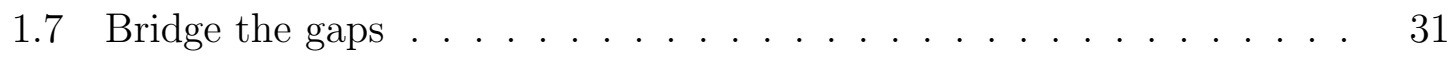

1.8 Research focus . . . . . . . . . . . . . . . . . . . . . 34

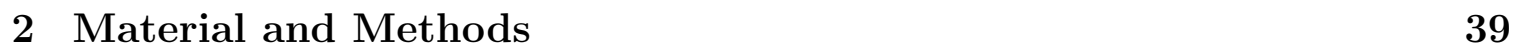

$2.1 \quad$ Data sources and inputs selection $\ldots \ldots \ldots \ldots$

$2.1 .1 \quad$ Mexico City . . . . . . . . . . . . . . . . . . . . . . . . . . 39

$2.1 .2 \quad$ Hong Kong . . . . . . . . . . . . . . . . . . . . . . . . 44

2.1 .3 Madrid . . . . . . . . . . . . . . . . . . . . 50

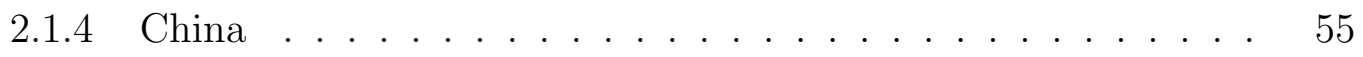

$2.1 .5 \quad$ Industry $\ldots \ldots \ldots \ldots \ldots \ldots \ldots \ldots$

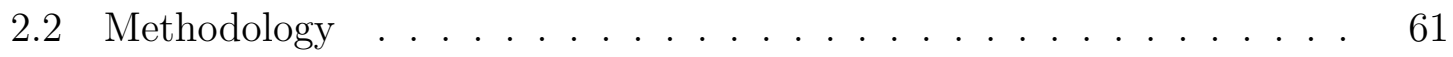

$2.2 .1 \quad$ Feature selection and reduction $\ldots \ldots \ldots$. . . . . . . 61 
$2.2 .2 \quad$ Preprocessing for imbalanced data $\ldots \ldots \ldots \ldots$. . . . . . 65

$2.2 .3 \quad$ Statistical modeling and machine learning . . . . . . . . . . 69

$2.2 .4 \quad$ Parameters tuning $\ldots \ldots \ldots \ldots \ldots \ldots$

2.2 .5 Cross validation $\ldots \ldots \ldots \ldots \ldots \ldots$

2.2 .6 Performance measurement . . . . . . . . . . . . . . . . 86

$2.2 .7 \quad$ Statistical comparison $\ldots \ldots \ldots \ldots \ldots$

$2.2 .8 \quad$ Cluster algorithm $\ldots \ldots \ldots \ldots \ldots$

2.2.9 Calculation for immaterialisation, dematerialization, and decarbonisation . . . . . . . . . . . . . . . . . . . . . 93

$2.3 \quad$ Tools and platform $\ldots \ldots \ldots \ldots \ldots \ldots$

$2.3 .1 \quad$ MySQL database $\ldots \ldots \ldots \ldots \ldots$

$2.3 .2 \quad$ ElasticSearch . . . . . . . . . . . . . . . . . . . 95

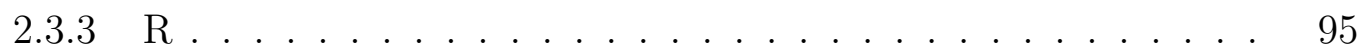

2.3 .4 Python . . . . . . . . . . . . . . . . . . . 96

2.3 .5 Stata . . . . . . . . . . . . . . . . . . 98

$\begin{array}{lll}3 & \text { State of the Art } & 99\end{array}$

$3.1 \quad$ Monitoring station re-configuration $\ldots \ldots \ldots \ldots$

3.2 Socio-economic activities factors: influencing on pollution . . . . . . . 101

3.3 Local short-term pollution forecasting . . . . . . . . . . . . . . . . 103

$3.3 .1 \quad$ Features selection techniques . . . . . . . . . . . . . . 105

$3.3 .2 \quad$ Multiple Linear Regression(MLR) . . . . . . . . . . . . . 106

3.3 .3 Time Series(TS) . . . . . . . . . . . . . . . . . . . . 107

$3.3 .4 \quad$ Artificial Neural Network(ANN) $\ldots \ldots \ldots \ldots$

$3.3 .5 \quad$ Support Vector Machine(SVM) $\ldots \ldots \ldots \ldots \ldots \ldots$

$3.3 .6 \quad$ Classification and Regression Trees(CART) . . . . . . . . . . . 112

3.3 .7 Ensemble Models . . . . . . . . . . . . . . . . . . . . . . . . 113

3.3 .8 Unbalance Techniques . . . . . . . . . . . . . . . . . . 115

3.4 Health evaluation personalization $\ldots \ldots \ldots \ldots \ldots$

$3.5 \quad$ Energy used awareness in production processes $\ldots \ldots \ldots \ldots \ldots$ 
3.5.1 Enhancing a sustainable production process by increasing energy awareness . . . . . . . . . . . . . . . . . . 121

3.5.2 Technological requirements for energy awareness . . . . . . . . 123

$3.6 \quad$ Energy-efficiency pattern analysis . . . . . . . . . . . . . . . . . . . . 124

4 Re-configure the Existing Monitoring Stations Network 127

4.1 Framework . . . . . . . . . . . . . . . . . . . . . . . 127

$4.1 .1 \quad$ Framework for reconfiguring . . . . . . . . . . . . . . . . . . . 127

4.1 .2 Research flow for the Mexico City case . . . . . . . . . . . . . 129

4.2 Description analysis . . . . . . . . . . . . . . . . . . . . . . 131

$4.3 \quad$ Feature extraction and selection . . . . . . . . . . . . . . . . . . 135

4.4 Model performance . . . . . . . . . . . . . . . . . . 137

4.5 Feature importance . . . . . . . . . . . . . . . . . . . . 142

4.6 Removing the stations with losing a minimum information . . . . . . 145

4.7 Results and discussion $\ldots \ldots \ldots$. . . . . . . . . . . . . 147

5 Forecast Ozone Levels: Dealing with Unbalanced Datasets 149

$5.1 \quad$ Identifying the potential influences of socio-economic activity factors on pollution . . . . . . . . . . . . . . . . . . . . . . 149

5.1 .1 Correlation analysis . . . . . . . . . . . . . . . . . . 150

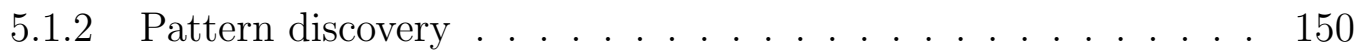

5.2 Research flow for forecasting . . . . . . . . . . . . . . . . . . 158

5.3 Description analysis $\ldots \ldots \ldots \ldots$. . . . . . . . . . . . . . . . 158

5.4 Forecasting . . . . . . . . . . . . . . . . 165

$5.4 .1 \quad$ Classification forecasting performance . . . . . . . . . . 165

5.4 .2 Regression performance . . . . . . . . . . . . 168

5.5 Importance of variables . . . . . . . . . . . . . . . . . . . . 171

5.6 Discussion . . . . . . . . . . . . . . . . . 175

6 Multi-level Awareness of Energy Used in Production Processes(Microcosmic) 179 6.1 Concept of multi-level energy awareness . . . . . . . . . . . . . . . . . 179 
6.2 Target: Energy used, its cost and $\mathrm{CO}_{2}$ emission . . . . . . . . . . . . 182

6.3 Requirements to enable multi-level energy awareness . . . . . . . . . 184

$6.3 .1 \quad$ Energy data . . . . . . . . . . . . . . . . . . . . 184

6.3 .2 Production data . . . . . . . . . . . . . . . . . . . . . . . . . . 185

6.3 .3 Technique requirements . . . . . . . . . . . . . . . 187

6.4 Pilot study . . . . . . . . . . . . . . . . . . . . . . . . . . 188

$6.4 .1 \quad$ Energy awareness at operation level . . . . . . . . . . . . . . . 189

6.4 .2 Energy awareness at product level . . . . . . . . . . . . . . . . 190

$6.4 .3 \quad$ Energy awareness at order level . . . . . . . . . . . . . . . . . 191

6.5 Discussion . . . . . . . . . . . . . . . . . . . . . . . 192

7 Driven Forces of Energy Consumption, GDP and $\mathrm{CO}_{2}$ Emission in

China

7.1 Description analysis . . . . . . . . . . . . . . . . . . . . . . . . . . . 195

7.2 Cluster analysis . . . . . . . . . . . . . . . . . . . . . . 197

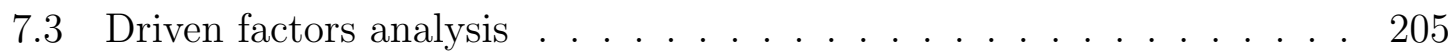

7.4 Discussion $\ldots \ldots \ldots \ldots \ldots$. . . . . . . . . . . . . . . . . . . 212

$\begin{array}{lll}8 & \text { Conclusion and Future work } & 217\end{array}$

8.1 Conclusion . . . . . . . . . . . . . . . . . . . . . . . . 217

8.2 Future work . . . . . . . . . . . . . . . . . . . . . . 220

\begin{tabular}{|ll}
\hline A Appendices & 225
\end{tabular} 


\section{List of Figures}

1-1 Relevant aspects of pollution, health impact, and energy . . . . . . . 23

2-1 Abstract of the research . . . . . . . . . . . . . . . . . 40

2-2 Location of the stations of the RAMA (Image courtesy of the Secretariat of Environment of Mexico City) . . . . . . . . . . . . . . . 41

$2-3$ Inputs and output for the models . . . . . . . . . . . . . . . . 44

$2-4 \quad$ Air quality monitoring network of HK. . . . . . . . . . . . . . 47

2-5 Candidate monitoring station selection for distance-based data set. . $\quad 49$

2-6 $\quad \mathrm{O}_{3}$ and $\mathrm{PM}_{2.5}$ levels distribution from 2014 to 2015 . . . . . . . . . . 52

2-7 $\quad$ Air quality monitoring network of Madrid . . . . . . . . . . . . . . 54

2-8 $\quad$ Individual tracking data in json file . . . . . . . . . . . . . . 55

$2-9$ Smart sensor for indoor pollution. . . . . . . . . . . . . 56

2-10 Application for Foobot. . . . . . . . . . . . . . 56

2-11 Beacons for identifying the nearest smart sensor. . . . . . . . . . 57

2-12 The map of provincial level administrative divisions of China. . . . . 57

$2-13$ FFBP structure . . . . . . . . . . . . . . . . . . . . . . . 78

2-14 Optimal hyper-plane in SVM(linearly seperable case) . . . . . . . . . 79

2-15 MySQL data base . . . . . . . . . . . . . . . . . . . . 95

2-16 ElasticSearch data base . . . . . . . . . . . . . . . . . . . . . . . . . . 96

3-1 representation of energy consumption of a sustainable-VSM [1] . . . . 122

4-1 Framework for reconfiguring an existing pollution monitoring network. 130

$4-2$ Research flow for the case study . . . . . . . . . . . . . . . . . . . 132 
4-3 Numbers of days that the daily maximum ozone level exceeded the limitation (0.11 ppm) against numbers of days when it did not exceed or equal the limitation at all stations by year: 2009, 2010, 2011, 2012. 133

4-4 Hourly average value corresponding to different variables at station

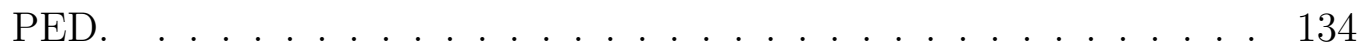

4-5 The hourly ozone levels of PED and its nearby stations at days 201005-02, 2010-05-03 and 2010-05-04. . . . . . . . . . . . . . . . . 134

4-6 The hourly ozone levels of PED and its far stations at day 2008-05-07, 2008-05-14 and 2008-05-19. . . . . . . . . . . . . . . . . . . . . . . . 135

4-7 Cumulative proportion of PCA corresponding to Datasets 1 to 4. . . 136

4-8 Errors of training data (internal) and validation dataset (external) (Dataset 3). . . . . . . . . . . . . . . . 136

4-9 Errors (RMSE) of numbers of variables corresponding to RFE _ RF (Dataset 3). . . . . . . . . . . . . . . . . . . . . . . . 138

4-10 Observed and predicted values of RF model for the temporal datasets 139

4-11 Predicted and observed values based on sampling size datasets . . . . 141

4-12 Heatmaps of Self-Organized Mapping according to variables. . . . . . 144

5-1 The inputs correlation for industry category. . . . . . . . . . . . . . . 150

5-2 $\quad$ The inputs correlation for manufacturing category. . . . . . . . . . . . 151

5-3 The inputs correlation for Meteorology categories. . . . . . . . . . . . 151

5-4 The inputs correlation for population and household categories. . . . 154

$5-5 \quad$ Abstract of the case study $\ldots \ldots \ldots$. . . . . . . . . . . . 159

$5-6 \quad$ Average level of $\mathrm{O}_{3}, R S P$ from year 1999 to 2007. . . . . . . . . . . . 160

5-7 Diurnal average ozone concentrations in Hong Kong at selected stations. . . . . . . . . . . . . . . . . . . 161

5-8 Frequency at which the daily maximum ozone appears at the corresponding time at the selected monitoring stations. . . . . . . . . . . . 162 
5-9 Number of days on which the limitation was exceeded versus the number of manufacturers from 1999 to 2007 (data sources: Census and Statistics Department of HK government) . . . . . . . . . . . . . . . 163

5-10 $\mathrm{O}_{3}$ levels at TW and YL stations from 2008-10-05 to 2008-10-07. . . . 163 5 5-11 Methodology overview. . . . . . . . . . . . . . . . 165

5-12 Predicted value versus observed value of distance-based data set. . . . 170 5-13 Ozone concentration levels from 2004 to 2012. . . . . . . . . . . . . . 171

5-14 The daily maximum ozone levels and its three additive components. . 172 5-15 Data after one lag differencing. . . . . . . . . . . . . . . . . . . . 172

5-16 ACF(top) and PACF(bottom) plots for one lag differenced adjusted daily maximum ozone data. . . . . . . . . . . . . . . . . . . . . . . . 173

5-17 Forecasts for the one lag difference adjusted daily maximum ozone level 174 5-18 Histogram of forecast errors. . . . . . . . . . . . . . . . . . . . . 174

5-19 Variable importance corresponding to SMOTE combining with CART, GBM, Bagging, and RF classifiers based on distance-based data set. . 176

6-1 Energy consumption by a machine for the production processes . . . 185

6-2 Class diagram for energy-aware production management . . . . . . . 186

6-3 Framework. . . . . . . . . . . . . . . . . . . . . . . . . . . . . 188

6-4 Cost of the energy used by operation (operation level) . . . . . . . . . 189

6-5 CO emission by product (product level) . . . . . . . . . . . . . . . . . 190

6-6 Energy consumption of product IDs 13, 14, 15 and 16 . . . . . . . . . 191

6-7 $\quad$ Energy distribution of products from order N1568. . . . . . . . . . . . 192

6-8 $\quad$ Energy distribution at order level, derived from the case study. . . . . 193

7-1 The 15 highest $\mathrm{CO}_{2}$ emission provinces in China at the year 1996, 2001,

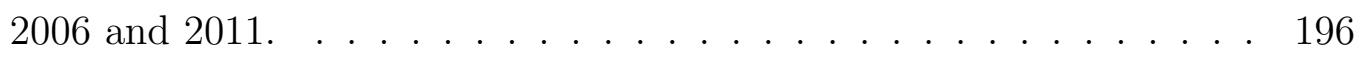

7-2 GDP corresponding to the selected most $\mathrm{CO}_{2}$ pollutant provinces in China from the year 1996 to 2011. . . . . . . . . . . . . . . . . . . . . 197

7-3 Chinas energy consumption structure in 2012. . . . . . . . . . . . . . 198

7-4 6 BIC for differences of Energy/GDP . . . . . . . . . . . . . . . 199 
7-5 $\quad$ AHC results GMM results for immaterialisation indicators . . . . . . 200

7-6 Indicator immaterialisation at provincial-level correspond to three periods based on AHC and GMM respectively . . . . . . . . . . . . . . 201

7-7 $\quad$ Energy changes corresponding to the provinces. . . . . . . . . . . . . 202

7-8 GDP changes corresponding to the provinces. . . . . . . . . . . . 203

7-9 Indicator dematerialization at provincial-level during three periods based on AHC and GMM respectively . . . . . . . . . . . . . . . . . . . . . 204

7-10 BIC for indicator dematerialization . . . . . . . . . . . . . . . . 205

7-11 Indicator decarbonisation at provincial-level correspond to three periods based on AHC and GMM respectively . . . . . . . . . . . . . . 206

7-12 AHC results against GMM results for the dematerialization indicator 207

7-13 Changes in the raw coal percentage of total energy in the three periods corresponding to different provinces. . . . . . . . . . . . . . . 209

7-14 The urbanization rate corresponding to different areas from year 2005

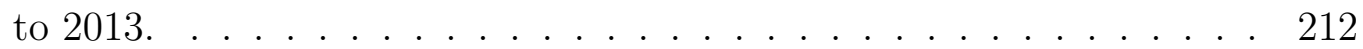

7-15 Top 1000 energy consumption enterprises corresponding to the provinces

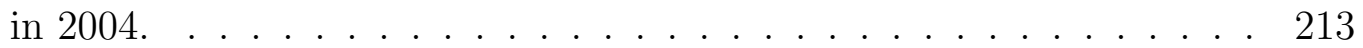

8-1 System design for individual pollution exposure evaluation. . . . . . . 223 


\section{List of Tables}

1.1 Sources category of pollutants . . . . . . . . . . . . . . . 24

1.2 The stakeholders and their concerns regarding energy awareness . . . 36

1.3 Energy awareness questions $\ldots \ldots \ldots \ldots \ldots \ldots$

$2.1 \quad$ Summary of inputs and output in the studies . . . . . . . . . . 43

2.2 Description of the variables of the different datasets . . . . . . . 45

$2.3 \quad$ Abbreviations of the air quality monitoring stations of HK. . . . . . 48

2.4 Description of input variables. . . . . . . . . . . . . . . 51

$2.5 \quad$ Input and output variables of single-site and distance-based data sets. 52

2.6 The names of provinces and their abbreviate $\ldots \ldots \ldots \ldots$

2.7 Details of an order f . . . . . . . . . . . . . . . . . . . . 59

2.8 The process $\log$ of machine 204 for Part Number A-118951 . . . . . 60

2.9 The process $\log$ of machine 204 for Part Number A-118951 . . . . . 60

2.10 Equations illustrating how the types of multi-level energy awareness can be calculated. . . . . . . . . . . . . . . . . . . . 62

2.11 Summary of merged data of process logging, energy consumption, cost of energy used and CO2 emission . . . . . . . . . . . . . . 63

2.12 The pseudocode for the integrating of Simulated Annealing . . . . . 66

2.13 The pseudocode for Recursive Feature Elimination. . . . . . . . . 67

2.14 Confusion matrix $\ldots \ldots \ldots \ldots \ldots$. . . . . . . . . . . 87

2.15 The pseudocode for Self-Organizing Map Algorithm . . . . . . . . . 91

2.16 Candidate models for selection . . . . . . . . . . . . . . . . . . 92

$2.17 \mathrm{CO}_{2}$ Conversion factors for each type of energy $\ldots \ldots \ldots . \ldots 9$ 
2.18 List of R Packages required $\ldots \ldots \ldots \ldots$

4.1 Pearson correlation matrix of the variables based on Dataset 3. . . . 137

4.2 Model performance MAE of Datasets 1 to $4 . \ldots \ldots \ldots$

4.3 Model performance RMSE of Datasets 1 to $4 . \quad \ldots \ldots \ldots$

4.4 Model performance RELE of Datasets 1 to $4 . \quad \ldots \ldots \ldots \ldots$

$4.5 \quad$ Results of Wilcox signed rank test to investigate statistically significant

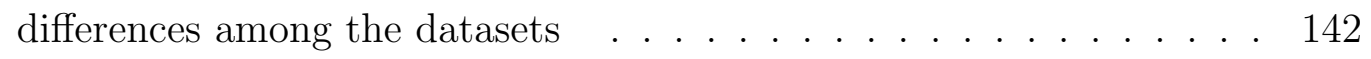

$4.6 \quad$ Results of Wilcox signed rank test based on MAE . . . . . . . . . . 143

$4.7 \quad$ RF model performance for time ahead prediction and various sizes of training data . . . . . . . . . . . . . . . . . . . 143

4.8 The rank of the first fifteen important variables by RF corresponding to Datasets 2, 3 and $4 \ldots \ldots \ldots \ldots \ldots$

4.9 Results of reducing the number of stations. . . . . . . . . 146

$5.1 \quad$ Fixed effected model . . . . . . . . . . . . . . . . . . . . 152

5.2 Random effected model . . . . . . . . . . . . . . . . . . . . 153

5.3 Hausman test . . . . . . . . . . . . . . . . . . . . . . . . 154

$5.4 \quad$ Fixed effects model with year dummy $\ldots \ldots \ldots \ldots \ldots$

$5.5 \quad$ Classification results corresponding to different sampling methods and AI techniques by adopting both single-site and distance-based data sets. 167

5.6 Outcomes of Wilcoxon matched-pairs signed-ranks test for measurement G_mean . . . . . . . . . . . . . . . . . . . . . . . . . 168

$5.7 \quad$ Averaged $G_{\text {mean }}$ over the three validation sets from distance-based data set corresponding to different thresholds . . . . . . . . . . . . 169

$5.8 \quad$ Performances of learning methods by threshold $0.32\left(78.525 \mu \mathrm{g} / \mathrm{m}^{3}\right.$ in actual ozone $) \quad \ldots \ldots \ldots \ldots \ldots \ldots$

6.1 Awareness level based on monitoring the level and factories orientation 180

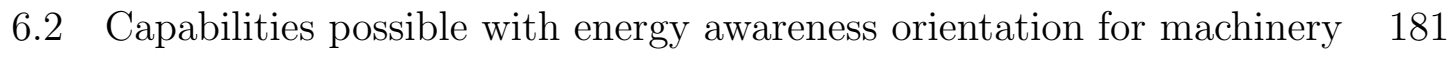

6.3 Capabilities possible with energy awareness orientation for products. 183 
$6.4 \quad$ CO emission by operation (operation level) $\ldots \ldots$. . . . . . . . . . . . 189

7.1 Changes in the percentage of added values of the primary, secondary, and tertiary sectors at provincial-level in China . . . . . . . . . . . . 208

7.2 Increasing rate of investment in coal mining and processing industry . 210

7.3 Increasing rate of investment in Energy Industry corresponding to different periods and provinces . . . . . . . . . . . . . . . . . . . . 211

7.4 Potential influential factors to $\mathrm{CO}_{2}$ emission. . . . . . . . . . . . . . . 214

7.5 Values of immaterialisation, decarbonisation and dematerialization for each province. . . . . . . . . . . . . . . . . . . . . 215

$1 \quad$ Input and out variables . . . . . . . . . . . . . . . . . . . . . 231 


\section{Chapter 1}

\section{Introduction}

\section{$1.1 \quad$ Background}

For many years, the air pollution problem has been attracting public, government and scholars' attention since it can cause serious health problems. The United States Environmental Protection Agency (EPA) set particle pollution (frequently referred to as particulate matter $)(\mathrm{PM})$, ground-level ozone $\left(\mathrm{O}_{3}\right)$, carbon monoxide $(\mathrm{CO})$, sulfur oxides $\left(\mathrm{SO}_{2}\right)$, nitrogen oxides $\left(\mathrm{NO}_{x}\right)$, and lead as 'critical pollutants'. Among these six pollutants, $\mathrm{PM}$ and $\mathrm{O}_{3}$ are the ones of the most dangerous pollutants for the whole social society [2]. Especially, they have adverse effects on public health, then leading to a significant economic loss [3, 4]. Some of the existing studies have evidenced that both short-term and long-term $\mathrm{PM}$ and $\mathrm{O}_{3}$ pollution exposure have a high correlation with respiratory and cardiovascular mortality and morbidity [5, 6, 7, 8, 9, 10]. Apart from the six pollutants, $\mathrm{CO}_{2}$ is one of the greenhouse gas being responsible for the global warming. The main contributor to $\mathrm{CO}_{2}$ is fossil fuels burning for providing energy to transportation, industry, agriculture, commercial and residential etc. Therefore, the issues related to energy, pollution, and pollution-related health impacts have sparked a considerable attention from the citizens, the governments, and an amount of researchers. 


\subsubsection{Environment}

Auditing, recording, and forecasting pollution are the effective ways and play essential parts in providing real-time or advanced pollution information to the public in order to avoid high pollution levels, as well as ensuring that the legal threshold has been enforced to lower the air pollution level and taking any appropriate action. Accordingly, they consist of the basic functions for the local pollution management system and are the ones of the most important events for the local relative authorities.

In fact, many cities around the world have built up their own monitoring networks. However, there are still two main issues in the existing network to be addressed sparsely or densely. The former one refers to the sparse network, which is sparsely distributed geographically that can not cover all the pollution variations in various areas, and should be augmented[11]. On the other hand, a dense network raises an issue regarding the stations' redundancy, which leads to a high cost of the measurement instruments and their maintenance. Consequently, the network design needs to be optimized[12].

To address the sparse or the dense distribution issues, an amount of the current studies put focus on the optimization of the whole air quality network, that is, relocating all the existing [13, 14, 12]. However, due to the limitation of available land space is a barrier that makes the new monitoring stations can not be placed at the exact location where the new network is proposed. The fully redesign, somehow, is hard to be realized. Furthermore, the cost is lower and the flexibility to consider different resign criteria(i.e information gain and error measurement) is higher when re-configure partly of the existing pollution monitoring network than fully redesign. The strategy

of the re-configuration, therefore, should be based on the problem situation that the current network exists.

Indeed, the accurate simulation or forecasting is the foundation stone for adjusting the new network configuration, since the quality of the spatial prediction in a network is a major criterion for modifying the existing one [15]. Since the 1970s, regression and auto-regressive models, such as time series analysis, have been widely used for fore- 
casting in the application of pollution prediction. However, the results suggested that traditional time series techniques fail to predict pollution accurately, especially for the pollutants that are formatted complicated such as ozone[16, 17]. As a replacement, Artificial intelligence(AI), which are the ones of the most sophisticated techniques, have shown and been proved their effectiveness and accuracy on prediction in many application areas, such as marketing[18, 19], financial[20, 21], health care[22, 23, 24], biological[25] and environment [26] etc. due to their capability of capturing non-linear characters or patterns from the history data. Also, they proved to be more effective for pollution prediction [27, 28]. Of the AI techniques, the support vector machine (SVM) [29, 30, 31, 32, 33], artificial neural network (ANN) [34, 30, 35], and decision tree (DT) [36, 37] are frequently employed in the domain of pollution forecasting. In recently years, with the successful application of the new AI techniques (i.e. ensemble

algorithms) in many domains, the accuracy prediction could be improved remarkably in the field of pollution and health domains.

Nevertheless, AI modeling is just one of the steps for achieving accurate prediction. Finding out the essential influencing factors, building up feature space, and selecting optimal features for modeling by various tools are necessary as well, which is an effective way to guarantee the model performances. The methods to select features and enhance the prediction performance are getting more versatile, making it deserve to be further trialled and discussed in the environmental modeling society.

\subsubsection{Public health evaluation}

With the rapid increment of modernization and industrialization in the cities, the public cannot fully avoid pollution exposure solely depending on pollution auditing and prediction. More awareness about the harm effects of pollution has been grown up among public and scholars due to the increasing life quality demand. Under such a situation, the health impact evaluation of pollution exposure is necessary for all the relevant stakeholders.

Regarding this topic, the relative concerns have been documented widely in the existing epidemiological studies [38, 39, 40, 41]. Traditionally, the evaluation of air 
pollution exposure has not been directly assessed for individuals, but rather for a group of people through annual average concentrations driven by the network of fixed monitoring stations.

Nevertheless, an increasing number of the citizens prefer to know "what is the pollution impact on me," making the tradition analysis of health impact unlikely to satisfy it. This is because the traditional analysis focuses on measuring the severe outcomes, of people who went to seek care but ignores the ones who have symptoms without awareness of the pollution related reactions, such as discomfort and irritability. In addition, the health effects were estimated by the average of contamination level in an area. This leads to underestimate variability in exposure [42. Under this circumstance, personalizing health evaluation is highly demanded by the citizens along with their requirement of improving living quality.

Thanks to the application of Internet of Thing(IoT), such as (mobile phones, wearable device ..), large volume and variety of data regarding the individual activities and behaviors, like human mobility and traffic flow, can be traced and recorded by second units. Most importantly, these factors like traffic volume are the primary sources of air pollution, can be used for improving the air quality modeling accuracy and making a specific evaluation of health impact. Even more, social web can be seen as "social sensor" and act as an important platform to gain information. The possible web sources can be personal health records, forums, blogs, search queries, video, images, tags, and rating, etc., from which a tremendous volume of individual information could be obtained [43]. These heterogeneous data make the fact possible, that is, customizing the pollution-related health evaluation.

Moreover, the application of social media has shown the effectiveness of forecasting real-world events, like election[44], flu outbreaks [45, 46], earthquakes [47, 48] and mental health trends[49]. A huge of volume and dynamical data regarding public responses to a particular event can be generated, which could be a "sensor" for that "event." In the domain of pollution, such "sensor" has already been evidenced by some studies [50, 51]. A high correlation between Air Quality Index(AQI) and daily frequency (volume) of original individual messages has been found. These studies 
are inspiring since such "social sensor" data can be used for health outcome forecasting and evaluation in the places where there is a lack of fully distributed air quality monitoring stations.

\subsubsection{Energy}

To achieve the goals of greenhouse gas reduction, all levels of stakeholders have spend efforts on either energy usage reduction or the energy efficiency improvement. From a micro point of view, the individual and manufacturer industry, for instance, tend to cut energy usage and improve energy efficiency in the way of utilizing sophisticated techniques and optimizing a managerial process. From a macro point of view, the national managers seek to enact proper policy to control individual energy usage behaviors.

Industry is responsible for $31 \%$ of primary global energy usage leading to $33 \%$ of global $\mathrm{CO}_{2}$ emissions [52]. Due to the rapid speed of industrialization, most companies are not immune to the combination of rising energy costs and environmental responsibilities, as well as the significant impact that regulatory compliance has on the effective day-to-day management of any organization. Therefore, lowering energy usage cost, improving energy efficiency, and reducing the $\mathrm{CO}_{2}$ emission in the process of production, have been devoted greater attentions from the stakeholders at different levels. Also, changing customers' behavior to greener products and complying with the new environmental regulations [53] have been seen as a key battleground to remain active and competitive for manufacturing organizations.

Although achieving a high-level awareness of the energy that is used during company operation is becoming essential for the adoption of more effective ways to improve energy efficiency, reduce its cost, and minimize environmental impacts from production processes, it still needs a comprehensive view of the energy system when making and implementing changes. Otherwise, the risk of sub-optimizing the energy system prevails [54]. Additionally, there will be different internal and external stakeholders who are looking for energy-related information. This means not only that strategic analyses of the energy-used need to be carried out, but also that there 
should be different levels of analyses, depending on the chosen perspective.

To address all of the needs, the energy-used at the process level is not sufficient. Aggregated information by machine, production line and factory, product and order are necessary. For this reason, a multi-level energy awareness at different managerial levels for all the stakeholders should be at the top of the agenda. This will also support decisions involving dynamic changes in production systems, resource availability, the environmental impact of production, and changing energy prices [55].

The same efforts should be spent on proposing feasible policies from both researchers and policy makers at the city, the province, the nation, the continent and the global levels as well. To deal with greenhouse gases emissions mitigation, adaptation and finance in the year 2020, Paris Agreement was negotiated by representatives of 195 countries at the $21^{\text {st }}$ Conference of the Parities of UNFCCC(United Nations Framework Convention on Climate Change) in Pairs on 12 December 2015. Until November 2015, 193 of UNFCCC members have signed the treaty. To achieve the goals, each country has initial their emission reduction target.

As the largest greenhouse gas emitter in the world, China has surpassed the United States and became the greatest $\mathrm{CO}_{2}$ emitter in the world. China accounts for $25 \%$ of the world carbon emission. A factor of 2.2 increasing of the primary energy was expected from the reference scenario of the International Energy Agency(IEA). By realization this issue, the Chinese government has taken a series of actions and regulation to deal with the climate change. In 2016, Chinese has announced the proposal 13th Chinas Five-Year Plans. This plan includes a series of and all aspects of social and economic development initiatives. One of the most crucial parts is to reduce greenhouse gas emissions, improve air quality and water quality. At 2009 Copenhagen Climate Summit, Chinese government commits to cut the intensity of its carbon emissions per unit GDP by 40-45\% by 2020 compared to 2005 level.

However, as one of the largest territory country, the patterns distribution of economic, energy consumption, and population and regions etc. in China are extremely different varying provinces, sectors, and regions. Therefore, a comprehensive reduction carbon emissions policy are needed to be proposed such as allocating national 
emission across provinces, in order to achieve the whole nation carbon emission target. Since the anthropogenic $\mathrm{CO}_{2}$ emissions are highly related to the socio-economic activities such as GDP and urbanization, it is necessary to analyze the $\mathrm{CO}_{2}$ emission patterns considering these factors as well, in which way the dynamic changes of energy usage and $\mathrm{CO}_{2}$ emissions can be fully connected to the social-economic activities.

According to the introduction aforementioned in the domain of pollution, its health impact, and energy, this study will narrowly focus on eight topics that are believed relevant as illustrated in Figure 1-1. More details and issues will be presented in the following subsections.

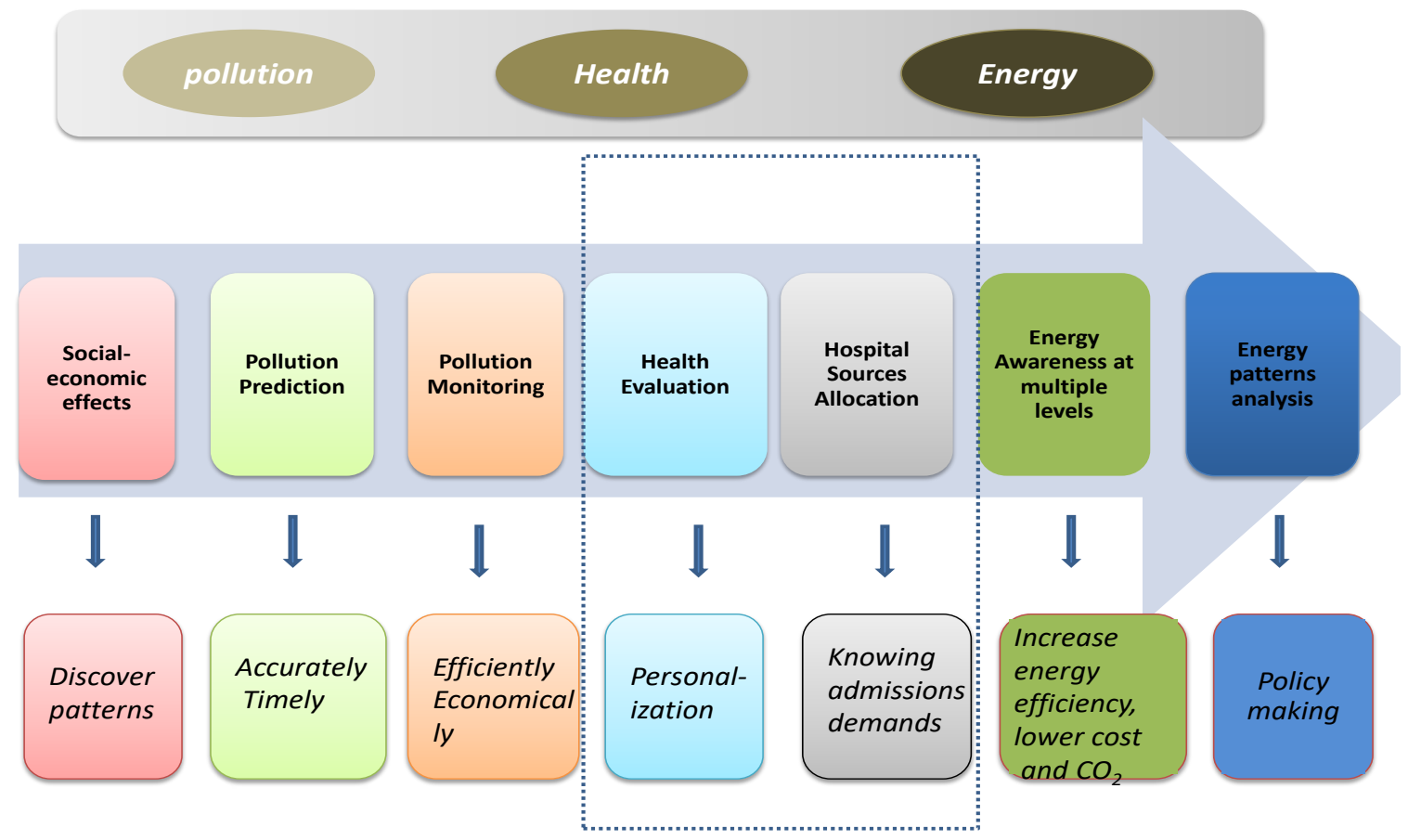

Figure 1-1: Relevant aspects of pollution, health impact, and energy

\subsection{Social-economic effects on pollution}

To better understand each pollutant and their formation patterns, some scholars have discovered the relationship between the emission sources and pollution. Among 
them, the sources from the manufacturing industry, and traffic and transport sectors get more attention than others. Unlike natural factors that are out of control, human activity is the key factor that is responsible for deteriorating air quality but under control. EPA classified the leading emissions into five sources, which are industrial point sources, utilities, non-road engines/vehicles, motor vehicles, and the area sources (Table 1.1). However, it is not sufficient to only know the pollution sources, the interaction of pollution and the detailed information about the sources should be obtained aiming to understand the behaviors of pollution formation better. One of the objects of this work, therefore, seeks to assess the socio-economic activity factors on the pollution at the sub-regional level in macro environments of Hong Kong. Then it will offer information for the policy and decision makers to formulate and develop an effective policy to control and reduce the emission.

Table 1.1: Sources category of pollutants

\begin{tabular}{lll}
\hline \hline Source category & Examples & Pollutants with sub- \\
& & stantial emissions re- \\
& & ductions from Clean \\
& & Air Act Amendments \\
& & compliance \\
\hline Electricity Generating Units & Electricity producing utilities & $N O_{x} / S O_{2}$ \\
Non-EGU Industrial Point Source & Boilers, cement kilns, process heaters, tur- & $N O_{x} / V O C / S O_{2}$ \\
On road Motor Vehicles & bines & \\
Non-road Engines/Vehicles & Buses, cars, trucks & $N O_{x} / V O C / P M / S O_{2}$ \\
& Aircraft, construction equipment, lawn & $N O_{x} / V O C / P M / S O_{2}$ \\
& and garden equipment, locomotives, ma- & \\
Area Sources & rine engines & $N O_{x} / V O C / P M$ \\
\hline
\end{tabular}




\subsection{Local Prediction}

\section{Importance of Feature Selection}

Feature construction and feature selection are the foundation stone and one of the most relevant steps for generating performing forecasting models. The research [56] emphasized that model architecture selection for artificial neural network is the best-implemented step, but more focus should be given to input selection considering input independence and model validation.

However, feature selection is frequently ignored, since the more the input features get correlated behavior with the dependence variable(pollution level), the easier the learning process is for the algorithm, and the better forecasting results can be obtained. Conversely, involving redundant or irrelevant features not only increases the noise and computation time but also probably degrades the performances of the models[57].

\section{Regional factors based on cases}

An advance knowledge and understanding of pollution formation can effectively help to select the relevant features. Therefore, it should be required before generating models. An amount of literature review is needed as the primary sources of such knowledge. Besides, a specific description analysis for a particular case is of great necessity for the decision of feature selection, since it can sometimes help to judge the core influence factors for the type of pollution. Nevertheless, lack of such preliminary description analysis is the main shortcoming and becoming an impeded for obtaining performance prediction results.

Regional factors, for instance, are the key points that could be considered as features. The selection of these factors highly depends on the characteristics of pollution formation varying cases, which can be judged by the description analysis before feature selection and modeling.

For instance, the study [58, 59] took Beijing an example and found that over 37 percentage of the cases that the deviation between the particular matter of the two stations at the same time of day are greater than $100 \mathrm{ppm}$. They believed that the 
current state of air quality of a location has not only temporal dependency on its current observations but also the state from past hours and its spatial neighbors. Thus, the usage of both local and regional pollution data could benefit forecasting, and help to enforce local pollution control actions and efficient abatement strategies in order to avoid a situation where all the cities are affected.

Regional models, such as the Community Multiscale Air Quality (CMAQ) model and the urban atmospheric dispersion model (DAUMOD), were proposed for ozone prediction in previous studies [60, 61]. However, the computation cost of these models is high, and it is not adequate for the application in urban areas where it presents severe photochemical pollution conditions. Conversely, AI modeling with regional factors as the inputs that could be characterized as fast computation and capacity to handle data including complex photochemical reaction and can be the ideal alternative tools for forecasting some complex formation pollutants, such as ozone.

\section{Models accuracy improved by ensemble models}

Since 2006, ensemble forecasting has begun to receive more attention in the data mining society, as ensemble algorithms can improve the forecasting accuracy and enhance the generalization capability [62]. Among these, boosting, bagging, and stacking are the most widely used ones and show promising pollution forecasting results [63, 64, 65]. A study reported in [66] revealed that a considerable improvement in the accuracy of forecasting $O_{3}$ is obtained by applying ensemble algorithms. As the ensemble models' inherent limitation associated with the single AI models, that is, the learning and training process still leads to a focus on and a bias toward to low-level concentrations (majority instances), these studies failed to forecast the "few events," i.e., the threshold exceedances of $\mathrm{O}_{3}$ concentrations, which constitutes an imbalanced data problem.

\section{Few events issues}

To circumvent this imbalance problem, researchers in the domain of data mining proposed various methods, among which data re-sampling, cost-sensitive learning, and algorithm modifications are widely used. Data re-sampling is a preprocessing method at the data level that modifies training instances to obtain relatively balanced samples 
before training the classifiers [67, 68, 69, 70]. Cost-sensitive learning incorporating both the data- and algorithm-level approaches is aimed to weight misclassification costs for instances in the minority class and thus lower the misclassification cost in the majority class and minimize high-cost errors [71, 35, 72]. Algorithm modification is performed at the algorithm level. The common strategy at the algorithm level is to choose an appropriate inductive bias, for instance, to adjust the class boundary based on the kernel alignment ideal for SVM [73, 69].

In applications for ozone forecasting, cost-sensitive learning with SVM and ANN, as well as algorithm modification methods, were adopted in [71, 35]. These studies proved the effectiveness of cost-sensitive and algorithm modification methods, but preprocessing methods were not discussed. Nevertheless, the application of a preprocessing technique to ensemble learning algorithms has proved to be effective in the data mining domain 74 .

\subsection{Air quality monitoring network reconfigura- tion}

The common way relies on simulation of pollutant concentrations by applying regional models that describe the chemistry transport [11, 75, 13] and geostatistical models i.e. Kriging algorithm, which aims at spatial prediction from observed value. This statistic method compared to the complex and intense computation regional simulation models, such as chemist-transport models, are simpler and time-saving. However,

kriging is an estimator that employed a linear combination of the observations or other factors. It is used for the network redesign that need interpolate the pollution value into the grids which cannot obtain the value directly from the monitoring stations. Nevertheless, it is hard to capture the complex formation characteristics for some pollutant like ozone.

From this perspective, a framework of methodology that employs the artificial intelligence techniques and information gathered is needed to be proposed. This 
framework can help the city environmental planners or managers to adjust the existing network configuration mainly focusing on the two situations: the requirement to move some stations and to install new stations.

The optimal network design of pollution monitoring can reduce the redundancy in an existing extensive monitoring network effectively. If the data from the one or some of current monitoring stations can predict pollution accurately at other monitoring station, the reduction of this monitoring station can be recommended to be removed from the monitoring network. These studies focus on how to resign the existing monitoring stations. The built models involves a specific complex regional modeling [11, 75], such as Chemical Transport Model(CMT) and industrial Sources Complex(ISC3) simulation tools[13]. These tools seek to trace the pollutants transport and transformation in the study area by using meteorological, emission inventory, and terrain information database, in such a way the simulation pollutant concentration level for each grid is generated. Besides, the statistic hourly mean, annual means of daily maximum 8-hr mean concentrations for $O_{3}$ were used to derive variograms for computing mean kriging variance for each grid. The comparison between simulated value and interpolated value are calculated and presented with root mean squared error(RMSE) or R-square $\left(R^{2}\right)$ as criteria for the optimized network decision.

The optimal air quality monitoring network can be obtained by considering multiobjectives, which includes environmental criteria, social criteria, and cost criteria. The sphere of influence(SOI) was employed to determine the minimum number of the sites required. It can be seen that majority of the existing studies using the regional models such as chemistry transport model [12] to simulate or forecast concentrations in the grid without ozone observation. The optimal network aims to ensure the ability to represent the pollution filed retrieved from the simulations maximize the coverage of the design domain which is measured by the distance between the network stations. 


\subsection{Energy used awareness}

Apart from six key pollutants identified by EPA, emission $\mathrm{CO}_{2}$ is a primary component of the greenhouse has obtained great attentions. The manufacturing industry counts for $33 \%$ of global $\mathrm{CO}_{2}$ emissions. Energy used information to all the relevant stakeholders based on their needs as illustrated in Table 1.2 is of great importance for controlling and reducing energy used, energy cost and $\mathrm{CO}_{2}$ emission.

Even some frameworks addressing the decision-making process related to energy management have been proposed[76, 777]. However, all of these proposals identify the benefits as the contribution at the corporate level and seek a degree of integration in the upper levels of the decision-making process. Therefore, energy awareness should be further enhanced to satisfy the needs of different stakeholders and is a fundamental and crucial step to control energy consumption.

The contribution can be particularly relevant when internal stakeholders want to connect energy consumption to maintenance policies or specific behaviors. Nevertheless, the emerging needs encourage the decision makers to take care of other concerns for all of the stakeholders. This may include providing green product information for customers [78, 79], identifying the amount and cost of energy used during processing operations and in products in order to improve production processes.

Therefore, a multi-level awareness of energy consumption helping to identify the amount of energy used, $\mathrm{CO}_{2}$ emitted, and the cost of the energy used at different levels such as operation, product, and order level, is needed to be proposed. This multi-level awareness, then, enables factories to answer the nine energy awareness questions that are presented in Table 1.3. These questions are grouped into three levels: energy-awareness at operational level, energy-awareness at product level, and energy-awareness at order level. The three groups also specify the energy used, the cost of the energy, and the $\mathrm{CO}_{2}$ emitted in production.

To enhance energy awareness by proposing different layers of information to satisfy the needs of different stakeholders, a framework is required to integrate collected energy data with production data. But the particular methodology or technological 
approach should be provided with sufficient detail to provide a guide for such detailed.

\subsection{Driven forces of dynamic changes: energy con- sumption, $\mathrm{CO}_{2}$ emission, and GDP}

At 2009 Copenhagen Climate summit, Chinese government committed to cut the intensity of its carbon emission per unit GDP by $40-45 \%$ by 2020 compared to 2005 level. To achieve the emission reduction target, local pollution abatement proposed is up to the agent and has been discussed by the government and academic community.

A study from [88] explored the regional characteristics of inter-provincial $\mathrm{CO}_{2}$ emissions and the regional distribution of the reduction of emission intensity reduction in China by K-means, Hierarchical clusters, and PSO-FCM clustering algorithms which is a method using the Particle Swarm Optimization(PSO) on Fuzzy-C means (FCM). To be different from the previous traditional studies that divided China based on regions: eastern, central, and western, the research from [88] examined how the regions corresponding to the divisions by economic developing level. Moreover, five indices including total emission, per capita emissions, $\mathrm{CO}_{2}$ emission per GDP (intensity of $\mathrm{CO}_{2}$ emission), $\mathrm{CO}_{2}$ emission per unit of energy and the proportion of industrial $\mathrm{CO}_{2}$ emission are considered to characterize $\mathrm{CO}_{2}$ emissions in China.

Research from [89] analyzed China's city level $\mathrm{CO}_{2}$ emission of energy consumptions by DMSP/OLS nighttime light imageries and explored major driving forces for proposing feasible mitigation policies. A recommendation is given to reduce the growth rate of $\mathrm{CO}_{2}$ without compromising economic development by optimizing the industrial structure of technology-intensive areas and improve energy efficiency in other areas.

The study [90], one the other hand, compares the transport development and energy use with a focus on $\mathrm{CO}_{2}$ emissions in the EU15 countries. Three terms related energy and emission were conducted, that is, immaterialisation, dematerialization, and decarbonisation. Immaterialisation refers to the reduction of energy intensity 
and transport intensity production and can be measured by indicator energy intensity $\Delta$ (energy/GDP); Dematerialization was defined as the reduction of carbon intensity of energy production and the carbon intensity of transport $\Delta\left(\mathrm{CO}_{2} /\right.$ energy $)$; Decarbonisation is the reduction in carbon of the whole economy, and it can be seen as a link between immaterialisation and dematerialization, which is measured by carbon intensity of economy $\left(\Delta\left(\mathrm{CO}_{2} / \mathrm{GDP}\right)\right.$. This study groups EU15 countries based on each of three indicators aforementioned in order to establish different patterns. The limitation of this study is that it lacks statistical analysis for identifying the main influential factors in each group.

A special IPAT identity case revealing the importance of distinct components or factors in the historical data of energy-related $\mathrm{CO}_{2}$ emission, which is often called Kaya identity, has been widely utilized in many studies [91, 92]. Research [91] decomposes urban emissions into four components(emission from primary, secondary, tertiary industry, and residential areas) to obtain a result of the interacting among 13 influential macro factors. 30 provinces in China were classified based on the historical data of the 13 macro factors. Shapley value decomposition was finally adopted to identify the key factors, in light of which the related policy control was promoted.

\subsection{Bridge the gaps}

In retrospective, the existing studies have empirically analyzed the local forecasting on pollution and pollution-related health impact issues, as well as some energy analysis. However, there are still some limitations, based on which further research can be extended. The main gaps are listed in the following six subsections.

\section{Gap 1: Ways for data selection}

First and foremost, the existing studies mainly focused on the pollution emission in the micro-environmental prospects, which aims to explore a particular sector activity, such as vehicular emission, manufacturer activities, or construction process. However, few comprehensive analyses about relations among all the sector activities and these pollutants from a macro-environmental view are explored, and as the pollutants are 
formatted in a complicated manner, all the factors of the emission sources could mutually be influenced and in the end affect pollution.

Furthermore, many of these previous studies revealed the correlation between the emission sources and the pollution from the energy and material consumption perspective. The energy consumption data of the possible emission sources are not available for some areas, including HK area. They conducted questionnaire surveys or make sampling studies by measurement emission directly by advanced instruments from sampling sites to obtain the data. However, this requires a highly technical training process and many material resources.

Therefore, how to use public open data sources to assess the risk socio-economic activity factors on the pollution at the sub-regional level in macro environments is one of the targets of this study. This can help us to identify the main factors that affect the HK local pollution and select the proper features for forecasting the pollution in an easy way. Also, this could also offer the information for the policy and decision makers to formulate and develop effective policies to control and reduce the emission.

\section{Gap 2: Description analysis before modeling}

For most local short-term pollution forecasting studies, the initial candidate features vary by different studies. There is not universal rule for the initial input selection. The features are more correlated with dependence variable; the learning process is easier for the algorithm and the better forecasting results can be obtained. Few studies focus on the features selection methods in the application of pollution. However, the proper combination of input features is the foundation stone for achieving promising forecasting results.

Nevertheless, a suitable combination of features should not be isolated from sufficient pollution analysis before they are finally chosen. The pollution formation patterns are different varying locations. Local pollution can be formatted by many reasons. It can be from sources emission, the chemical reaction among emissions, and regional factors like pollution transportation. Therefore, if there are not preliminary analysis for the pollution, the features cannot be collected properly and lead to inaccuracy forecasting in the end. 


\section{Gap 3: Framework of re-configure existing network}

The sparse or dense network can be judged from the pattern observations of pollution level that is recorded by the various air quality monitoring stations in the local area. Then, a comprehensive pollution data analysis among different monitoring stations are required to judge the regional characteristics of local pollution formations and to make the decision if updating the existing monitoring stations is needed. Moreover, due to the lack of a framework for reconfiguring or upgrading the existing monitoring network, it needs to provide such a framework for the city managers in their future work.

\section{Gap 4: Forecasting high-level concentrations of the pollutants}

In the community of data mining, the ensemble algorithms have been acting an important role in the forecasting, some of which have been employed in many applications, including pollution modeling. However, there is no evidence showing the ensemble models can help to improve the prediction of the "few events" mentioned before, since the ensembles always inherit the disadvantages of single machine learning algorithms. Therefore, it is of great importance to combine the existing data mining algorithms with multiple methods for a particular research purpose.

\section{Gap 5: Energy used awareness at multiple levels}

The existing studies usually focus on the awareness of the whole manufacturing industry and benefits at department level, and less industry energy management attention has been given to providing environmental information on the specific product to customers. Furthermore, the previous studies that create energy awareness in the production process limit the scope for improving efficiency in the production of products, as they mainly support the operators decision. This way cannot benefit further from an integration of the analysis of the entire production process since one product and one order are not completed by only one machine, but by cooperation between several machines. Moreover, a lack of holistic orientations of energy awareness is an obstacle to decision-making for stakeholders.

Furthermore, even frameworks have been proposed to integrate collected energy data with production data, although no particular methodology or technological ap- 
proach has been provided with sufficient detail to provide guidance for such detailed, but still critical, levels of information. This study strives to enhance energy awareness by proposing different layers of information to satisfy the needs of different stakeholders.

Gap 6: Provincial energy-efficiency patterns from dynamics indicators

The main limitation of the existing is that either lacking dynamic view to reveal the changes of China's energy usage and $\mathrm{CO}_{2}$ emissions or fail to use models to identify the main important factors for different dynamic indicators. To bridge the gap of the current research, this study aims to dynamically analyze the patterns of energy usage and $\mathrm{CO}_{2}$ emission at the provincial level by utilizing the indicators, imiaterialisation, dematerializaion and decarbonisation and adopting various cluster algorithms. Moreover, a statistic analysis via potential influential factors will be taken into account to find out the main factors for each category based on the clustering result. Finally, the outcome of the results can help understand the driving factors and benefit to the policy makers to promote feasible policies.

\subsection{Research focus}

To fill these gaps, this monograph promotes specific methods based on several case studies to illustrate how to use the artificial intelligence better. The rest of this study consists of the following parts:

- Chapter 2 will provide a view for the material and methods. It includes the data sources for the cases, and feature selection and artificial intelligence techniques that will be employed in this study.

- The state-of-the-art will be introduced in Chapter 3 to deep insight the current research background and trend in the application of prediction by artificial intelligence techniques.

- The air quality monitoring station network has been identified as dense via 
descriptive analysis in Chapter 4. A methodology of air quality monitoring network reconfiguration is proposed. The realization of this methodology is based on the information gathered from prediction results. Chapter 4 also shows how the effectiveness of local pollution forecasting models is established by the feature selection and AI techniques(Gap 2 and 3).

- Chapter 5 mainly includes two parts. The first part will depict the results of identifying the socio-economic factors on various pollutants that related to Gap 1. The finding for the particular pollutant, ozone, as a result is used for the threshold exceedances forecasting in the second part, which is related to Gap 4. The second part will present how the ensemble algorithm and pre-processing techniques can address the imbalance class issues and improve the forecasting results through Hong Kong case. Meanwhile, regional factors were considered by using a regional scale data set and assessing its empirical relevance(Gap 1 and 4).

- One of this monograph is to propose multi-level energy awareness that focuses on the products production process to provide energy and environmental information for energy management by the industry, customers and governments in order to satisfy their need for a higher level of energy awareness. This will be introduced by a pilot study in Chapter 6(Gap 5).

- A cluster analysis of energy efficiency is presented in Chapter 7by using dynamic indicators showing the changes of energy efficiency in China(Gap 6).

- In the end, the conclusion will be given, and the future work including undergoing and coming research activities are included in Chapter 8. 
Table 1.2: The stakeholders and their concerns regarding energy awareness

\begin{tabular}{|c|c|c|}
\hline Stakeholders & Main Concerns regarding energy & References \\
\hline $\begin{array}{l}\text { Operators } \\
\text { (factory level) }\end{array}$ & Best practices assessment;Event identification. & 80 \\
\hline $\begin{array}{l}\text { Energy managers } \\
\text { (factory level) }\end{array}$ & $\begin{array}{l}\text { Priority rebalances regarding energy consump- } \\
\text { tion;Energy audits. }\end{array}$ & 81 \\
\hline $\begin{array}{l}\text { Quality manager } \\
\text { (factory level) }\end{array}$ & $\begin{array}{l}\text { Energy metering as useful insight for lean prac- } \\
\text { tices;Quantitative identification of waste. }\end{array}$ & 82 \\
\hline $\begin{array}{l}\text { Production manager } \\
\text { (factory level) }\end{array}$ & Integrated KPI view, including energy KPIs. & 83 \\
\hline $\begin{array}{l}\text { Industry } \\
\text { (factory level) }\end{array}$ & Continuous improvement of energy goals. & [84, 1] \\
\hline Customers & $\begin{array}{l}\text { The green information of products; Transparency } \\
\text { regarding the products entire value chain. }\end{array}$ & 85 \\
\hline (market level) & & \\
\hline $\begin{array}{l}\text { Governments } \\
\text { (regulatory level) }\end{array}$ & Energy usage performance & 86 \\
\hline Society & Emissions. & \\
\hline Provider & $\begin{array}{l}\text { Transparency of energy aspects for components } \\
\text { and raw materials (information flow). }\end{array}$ & 87 \\
\hline
\end{tabular}


Table 1.3: Energy awareness questions

\section{Energy-aware at operation level.}

1. How much energy is consumed for processing per unit of operation $\mathrm{X}_{i}$ ?

2. How much is the cost of the energy used in processing per unit of operation $\mathrm{X}_{i}$ ?

3. What is the volume of $\mathrm{CO}_{2}$ emissions that are produced in processing a unit of operation $\mathrm{X}_{i}$ ?

Energy-aware at product level.

4. How much energy is consumed to produce product $\mathrm{P}_{i}$ ?

5. How much is the cost of the energy used to produce product $\mathrm{P}_{i}$ ?

6. What is the volume of $\mathrm{CO}_{2}$ emissions that are produced by producing product $\mathrm{P}_{i}$ ?

Energy-aware at the per order level.

7. How much energy is consumed in producing order $\mathrm{O}_{i}$ ?

8. How much is the cost of the energy used to produce order $\mathrm{O}_{i}$ ?

9. What volume of $\mathrm{CO}_{2}$ emissions is produced in producing order $\mathrm{O}_{i}$ ?

\section{Notations}

$\mathrm{X}_{i}$ : elementary operation of a process $i . \mathrm{P}_{i}$ : product $i . \mathrm{O}_{i}$ : order $i$ 


\section{Chapter 2}

\section{Material and Methods}

To fill the gaps mentioned in Chapter 1, six study cases will be presented. Each case corresponds to a focus in Figure 1-1. The data sources, variable selection, the state-of-the-art statistics and artificial intelligence techniques, and the performance measurements are included in this section. At the end of this chapter, the tools, including software and platform are summarized.

To better understand the targets of each study and their corresponding methodologies adopted, a framework is summarized graphically in Figure2-1, where it covers four aspects including research targets, applied cases, modelling, and feature selection and data integration.

\subsection{Data sources and inputs selection}

\subsubsection{Mexico City}

\section{Data Sources}

The Valley Metropolitan Area of Mexico (ZMVM, its Spanish acronym) is a highlands plateau in central Mexico and the eastern half of the State of Mexico. It occupies 7854 $\mathrm{km}^{2}$, of which $1500 \mathrm{~km}^{2}$ are already urbanized. It includes 16 boroughs that comprise 60 municipalities. Its elevation is 2,240 $\mathrm{m}$ above sea level. The area is surrounded by

high mountains and located in the subtropical region with intensive solar radiation 


\begin{tabular}{|c|c|c|c|c|c|c|c|}
\hline $\begin{array}{l}\text { Research } \\
\text { targets }\end{array}$ & $\begin{array}{l}\text { Identify } \\
\text { socio- } \\
\text { economic } \\
\text { activity } \\
\text { factors }\end{array}$ & $\begin{array}{l}\text { Reconfigur } \\
\text { e existing } \\
\text { monitorin } \\
\text { g network }\end{array}$ & $\begin{array}{l}\text { Forecast } \\
\text { high level } \\
\text { pollution }\end{array}$ & $\begin{array}{l}\text { Forecast } \\
\text { hospital } \\
\text { admissions }\end{array}$ & $\begin{array}{l}\text { Individual- } \\
\text { health } \\
\text { exposure } \\
\text { evaluation }\end{array}$ & $\begin{array}{l}\text { Multi-level } \\
\text { energy } \\
\text { awareness }\end{array}$ & $\begin{array}{l}\text { Discover } \\
\text { the } \\
\text { patterns of } \\
\text { energy, } \mathrm{CO}_{2} \\
\text { GDP }\end{array}$ \\
\hline Cases & Hong Kong & Hong Kong & Mexico City & Madrid & Madrid & Industry & China \\
\hline Modelling & $\begin{array}{l}\text { Statistic } \\
\text { analysis } \\
\text { (Panel data, } \\
\text { Correlation } \\
\text { analysis) }\end{array}$ & $\begin{array}{l}\text { Preprocess } \\
\text { ing tools, } \\
\text { single and } \\
\text { ensemble } \\
\text { Als }\end{array}$ & $\begin{array}{l}\text { Feature } \\
\text { selection(PC } \\
\text { A, SA, RFE), } \\
\text { Als(MLR,ANN } \\
\text {, RF), } \\
\text { cluster(SOM) }\end{array}$ & $\begin{array}{l}\text { Single and } \\
\text { ensemble } \\
\text { Al models }\end{array}$ & $\begin{array}{l}\text { Geographic } \\
\text { Statistic } \\
\text { modelling } \\
\text { (Kriging) }\end{array}$ & $\begin{array}{l}\text { Processing } \\
\text { analysis }\end{array}$ & $\begin{array}{l}\text { Cluster } \\
\text { (AHC, } \\
\text { GMM) }\end{array}$ \\
\hline $\begin{array}{l}\text { Feature } \\
\text { selection } \\
\text { and data } \\
\text { integration }\end{array}$ & $\begin{array}{l}\text { Traffic flow, } \\
\text { industry, } \\
\text { household } \\
\text { from public } \\
\text { database, } \\
\text { meteorologic } \\
\text { al and } \\
\text { pollution data } \\
\text { from } \\
\text { monitoring } \\
\text { stations }\end{array}$ & $\begin{array}{l}\text { Pollution } \\
\text { and } \\
\text { meteorolo } \\
\text { gical data } \\
\text { from } \\
\text { outdoor } \\
\text { monitoring } \\
\text { stations }\end{array}$ & $\begin{array}{l}\text { Pollution and } \\
\text { meteorologic } \\
\text { al data from } \\
\text { monitoring } \\
\text { stations }\end{array}$ & $\begin{array}{l}\text { Hospital } \\
\text { admissions } \\
\text { Tweets } \\
\text { from } \\
\text { twitter, } \\
\text { meteorolo } \\
\text { gical and } \\
\text { pollution } \\
\text { data }\end{array}$ & $\begin{array}{l}\text { Meteorologi } \\
\text { cal and } \\
\text { pollution } \\
\text { data from } \\
\text { indoor } \\
\text { smart } \\
\text { sensors, } \\
\text { and from } \\
\text { outdoor } \\
\text { monitoring, } \\
\text { Individual } \\
\text { tracking } \\
\text { information } \\
\text { from smart } \\
\text { phone }\end{array}$ & $\begin{array}{l}\text { Production, } \\
\text { machine } \\
\text { configuring } \\
\text { data from } \\
\text { manufacturing, } \\
\text { energy } \\
\text { consumption } \\
\text { from smart } \\
\text { sensors, } \\
\text { emission } \\
\text { conversions }\end{array}$ & $\begin{array}{l}\text { Energy } \\
\text { used from } \\
\text { China year } \\
\text { notebook, } \\
\text { emission } \\
\text { conversion } \\
\text { factors } \\
\text { from IPCC, } \\
\text { other } \\
\text { economic } \\
\text { data from } \\
\text { public data } \\
\text { sources }\end{array}$ \\
\hline
\end{tabular}

Figure 2-1: Abstract of the research 
throughout the year. There are 20 million inhabitants, more than 53,000 industries and 3.5 million vehicles. All of these favor the creation of ozone pollution.

In this study, all pollutants and meteorology data have been selected from the Secretariat of Environment Administering the Atmospheric Monitoring System of the City of Mexico (SIMAT, its Spanish acronym). SIMAT is composed of an Automatic Atmospheric Monitoring Network (RAMA), which has 39 stations, a Network Air Quality Monitoring Manual (REDMA) that has 12 stations, the atmospheric deposition network (REDDA), which has 16 sampling site, and the Network of Meteorology and Solar Radiation (REDMET), which operates 16 stations. Figure 2-2 shows the locations of the stations of the RAMA. All values are recorded hourly and expressed in parts per million.

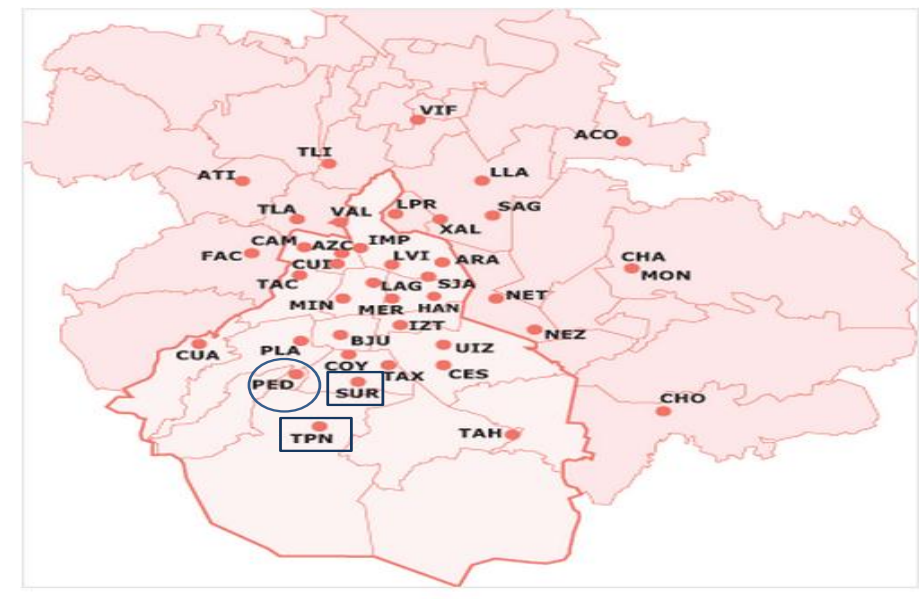

Figure 2-2: Location of the stations of the RAMA (Image courtesy of the Secretariat of Environment of Mexico City)

Source:

escritorio/mapas.php

(SIMAT),http://www.calidadaire.df.gob.mx/calidadaire/

\section{Preparation of the datasets}

In light of the discovery in previous studies of the mechanism of its formation [30, 72, ozone is formed mainly by the primary emission of compounds, such as nitrogen monoxide $\left(\mathrm{NO}_{x}\right)$, nitrogen dioxide $\left(\mathrm{NO}_{2}\right)$ and volatile organic compounds (VOCs), under meteorological conditions, such as TMP and relative humidity (RF), and is 
possibly is influenced by the wind speed (WSP) and wind direction (WDR) [93, 94]. Moreover, considering the possible correlation between ozone and other factors such as carbon oxide $(\mathrm{CO})$ and sulfur dioxide $\left(\mathrm{SO}_{2}\right)$ (Figure 4-4), and the time of day (Figures 4-5 and 4-6), this study employs $\mathrm{O}_{3}, \mathrm{NO}_{x}, \mathrm{NO}_{2}, \mathrm{NO}_{x} / \mathrm{NO}_{2}, \mathrm{CO}, \mathrm{SO}_{2}, \mathrm{RH}$, TMP, WDR, WSP, Month (M), Season (S), date (D), and hour (H) as the candidate feature space. Based on these, the maximum, minimum, average, median and sum values of three rolling hours for each candidate feature were generated.

Later, five categories of datasets were generated for different objectives as illustrated in Table 1. These categories were single-site, regional-site, support, temporalbased, and sample-size data sets, The single-site and regional-site datasets are used primarily for model selection and ozone monitoring station reconfiguration, whereas the temporal-based and sample-size data sets are used for determining the sensitivity against prediction lags and sample size.

The single-site dataset is based only on the data from the PED station, including Dataset 1 and Dataset 2. The regional-site datasets include Datasets 3 to 10, where the inputs were generated only from the TPN, PED, and SUR stations.

In more detail, Dataset 3 was generated firstly, for the purpose of having a fair comparison. The inputs of Datasets $1,2,4,5,6,7,8,9$, and 10 are various combinations of the features that were extracted from Dataset 3, but with the same training and testing sample size. Thus, a total of 2194 samples are obtained, of which 2000 were selected for training and validation and 194 for testing.

For the temporal-based category datasets, five new data sets, namely - 2-p2, 2p3, 2-p4, 2-p5 and 2-p6 were included. These datasets were prepared with the same features, but forecasting ozone at different time lags (from 2 hours in advance to 6 hours in advance). The features of these datasets are the same as those of Dataset 2. However, we reselected and merged the data only from the PED station in order to obtain more samples. Finally, more than 56,000 samples were generated. From these samples, 2000 were selected randomly as training and validation sets and 1000 samples as the testing set.

For the sampling-size category, four more datasets that were based on Dataset 
2-p2 (Dataset 2-s2000) were proposed. They were 2-s4000, 2-s8000, and 2-s16000 with 2000, 4000, 8000 and 16,000 samples, respectively and used to test the effect of the training samples on the model accuracy. The categories, number of inputs and locations of the sources, output, and the usage goals of each dataset can be found in Figure 2-3 and Table 2.1.1.

Then, all the variables were normalized by Equation 2.1. In order to deal with model over-fitting, 5-fold cross-validation, repeated two times, was applied.

Table 2.1: Summary of inputs and output in the studies

\begin{tabular}{|c|c|c|c|}
\hline Studies & Inputs & Output & Target Place \\
\hline 95 & $\begin{array}{l}\text { dummy variable(holiday versus working day) } \\
\mathrm{O}_{3} \text { at 9:00, maximum TMP, } \\
\text { average of }\left(\mathrm{CO}_{2}, \mathrm{NO}_{2}, \mathrm{NO}_{x}, \mathrm{WSP}, \mathrm{WDR}\right) \\
\text { between 6:00 a.m. and 9:00 a.m }\end{array}$ & daily maximum $\mathrm{O}_{3}$ levels & Dallas-Fort \\
\hline 96 & daily average $\mathrm{O}_{3}, \mathrm{RH}, \mathrm{SR}$, rainfall(RF), WSP & daily average $\mathrm{O}_{3}$ & Campo Grande, Brazil \\
\hline [16] & $\begin{array}{l}\text { daily maximum temperature (TMP) } \\
\text { average daily dew point temperature (DT) } \\
\text { average daily WSP } \\
\text { and daily total sunshine }() \\
\text { maximum } 1 \text { hour } \mathrm{O}_{3} \text { from the previous day }\end{array}$ & daily maximum 1-hour $\mathrm{O}_{3}$ & Eight sites at USA \\
\hline 97 & $\begin{array}{l}\left(\mathrm{CH} 4, \mathrm{NMHC}, \mathrm{CO}, \mathrm{CO}_{2}, \mathrm{NO}, \mathrm{NO}_{2} \text { and } \mathrm{SO}_{2}\right) \\
\text { WSP, WDR, TMP, RH, SR })\end{array}$ & hourly $\mathrm{O}_{3}$ & Kuwait University \\
\hline 98 & $\begin{array}{l}\text { solar flux, the hour and the month } \\
\text { WDR, wind velocity (WV) } \\
\text { TMP, RH, } \mathrm{SO}_{2}, \mathrm{CO}, \mathrm{O}_{3}, \mathrm{NO}_{2}, \mathrm{NO}_{x}, \mathrm{NO}_{x} / \mathrm{NO}_{2}\end{array}$ & hourly $\mathrm{O}_{3}$ & Mexico city \\
\hline 99 & $\begin{array}{l}\mathrm{O}_{3}, \mathrm{CO}, \mathrm{NO}_{2}, \mathrm{SO}_{2}, \mathrm{TMP} \\
\mathrm{RH}, \mathrm{WSP}, \mathrm{WDR}, \mathrm{H}\end{array}$ & hourly $\mathrm{O}_{3}$ (classification) & Mexico City \\
\hline
\end{tabular}




$$
\text { newInput }=\frac{\text { input }-\min (\text { inputs })}{\max (\text { inputs })-\min (\text { inputs })}
$$

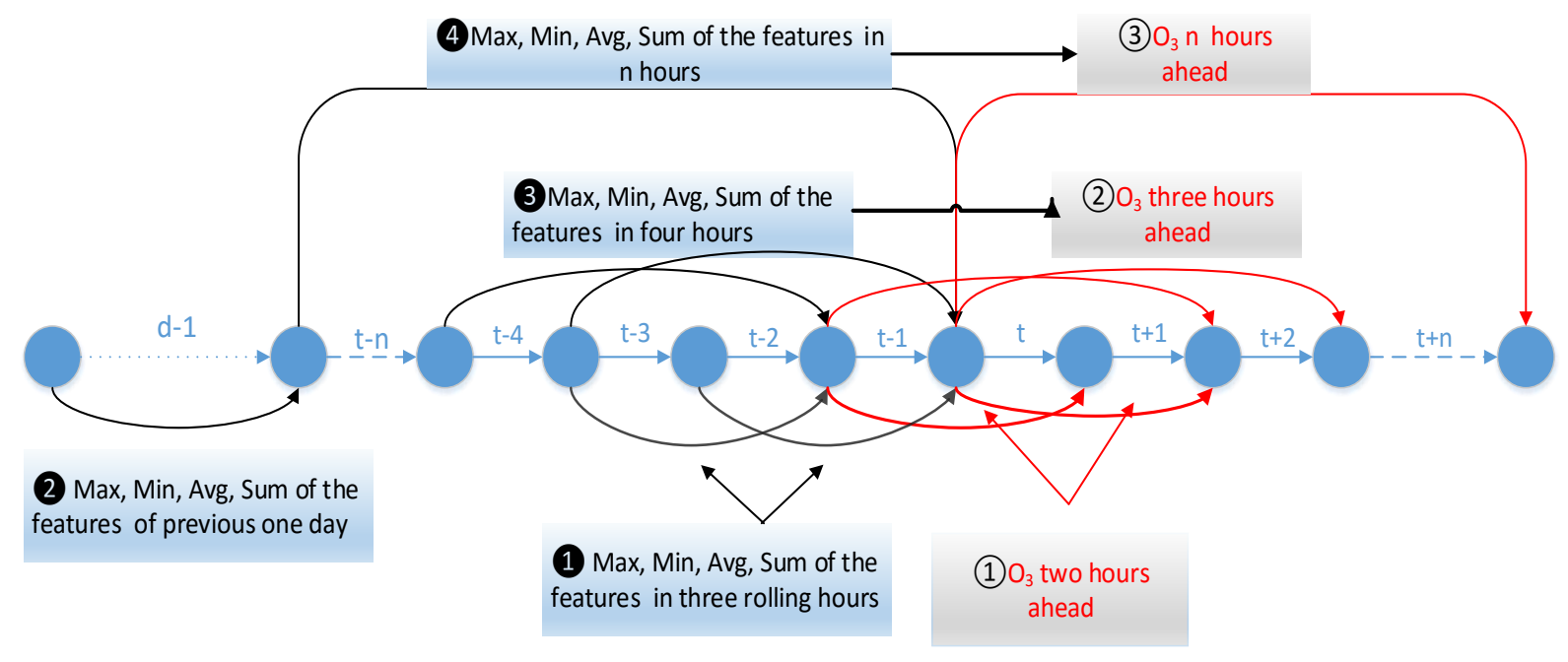

Features include $\mathrm{O}_{3}, \mathrm{NO}_{x}, \mathrm{NO}_{2}, \mathrm{NO}_{x} / \mathrm{NO}_{2}, \mathrm{CO}, \mathrm{SO}_{2}, \mathrm{RH}, \mathrm{TMP}, \mathrm{WDR}, \mathrm{WSP}$

Description features:Month(M), Day(D), Season(S),Hour(H)

Figure 2-3: Inputs and output for the models

\subsubsection{Hong Kong}

\section{Data Sources}

HK is located on the south coast of mainland China, close to the PRD area, which is one of the most developed in China. The entire territory of HK consists of the HK Island, the Kowloon peninsula, New Territories, and over 200 small islands. Its terrain is hilly to mountainous with steep slopes [100]. HK is one of the most densely populated areas in the world, with a population of 7.155 million and a surface area of 
Table 2.2: Description of the variables of the different datasets

\begin{tabular}{|c|c|c|c|c|c|}
\hline Category & Datase & $\begin{array}{l}\text { No.of } \\
\text { puts }\end{array}$ & Inputs & Output & Purpose \\
\hline \multirow[t]{2}{*}{ Single-site } & 1 & 55 & (1) at PED & $\begin{array}{l}2 \mathrm{~h} \text { in advance } \\
\mathrm{PED}\end{array}$ & $\begin{array}{l}\text { To determine if the local pollution and } \\
\text { weather conditions of the present day can } \\
\text { forecast the ozone level at PED two hours } \\
\text { in advance }\end{array}$ \\
\hline & 2 & 95 & (1+2 at PED & $\begin{array}{l}2 \mathrm{~h} \text { in advance } \\
\mathrm{PED}\end{array}$ & $\begin{array}{l}\text { To determine if the previous day of local } \\
\text { pollution and weather conditions can affect } \\
\text { the present days ozone levels }\end{array}$ \\
\hline \multirow[t]{2}{*}{$\begin{array}{l}\text { Regional- } \\
\text { site }\end{array}$} & 3 & 275 & $\begin{array}{l}\mathbf{1}+\mathbf{2} \text { at PED, } \\
\text { SUR, and TPN }\end{array}$ & $\begin{array}{l}2 \mathrm{~h} \text { in advance } \\
\mathrm{PED}\end{array}$ & $\begin{array}{l}\text { To determine if the pollution and weather } \\
\text { conditions of neighboring stations can af- } \\
\text { fect the local PED ozone level }\end{array}$ \\
\hline & 4 & 185 & $\begin{array}{l}\mathbf{0}+2 \text { at SUR and } \\
\text { TPN }\end{array}$ & $\begin{array}{l}2 \mathrm{~h} \text { in advance } \\
\mathrm{PED}\end{array}$ & $\begin{array}{l}\text { To determine if only the pollution and } \\
\text { weather conditions of neighboring stations } \\
\text { can forecast the ozone level at the local } \\
\text { PED station }\end{array}$ \\
\hline \multirow[t]{6}{*}{ Support } & 5 & 95 & $\mathbf{1}+2$ at PED & $\begin{array}{l}2 \mathrm{~h} \text { in advance } \\
\text { SUR }\end{array}$ & $\begin{array}{l}\text { Used for the ozone monitoring stations re- } \\
\text { duction }\end{array}$ \\
\hline & 6 & 95 & (1+2 at TPN & $\begin{array}{l}2 \mathrm{~h} \text { in advance } \\
\text { SUR }\end{array}$ & For station reduction \\
\hline & 7 & 95 & (1) at SUR & $\begin{array}{l}2 \mathrm{~h} \text { in advance } \\
\text { SUR }\end{array}$ & For station reduction \\
\hline & 8 & 95 & (1+2 at PED & $\begin{array}{l}2 \mathrm{~h} \text { in advance } \\
\mathrm{TPN}\end{array}$ & For station reduction \\
\hline & 9 & 95 & (1+2 at SUR & $\begin{array}{l}2 \mathrm{~h} \text { in advance } \\
\mathrm{TPN}\end{array}$ & For station reduction \\
\hline & 10 & 95 & $\boldsymbol{1}+\mathbf{2}$ at $\mathrm{TPN}$ & $\begin{array}{l}2 \mathrm{~h} \text { in advance } \\
\mathrm{TPN}\end{array}$ & For station reduction \\
\hline $\begin{array}{l}\text { Temporal- } \\
\text { based }\end{array}$ & $2-\mathrm{p}(\mathrm{n})$ & 95 & 3,4 at $\mathrm{PED}$ & $\begin{array}{l}n \mathrm{~h} \text { in advance } \\
\mathrm{PED}\end{array}$ & $\begin{array}{l}n \text { is from } 2 \text { to } 6 \text {. Used for testing the mod- } \\
\text { els sensitivity to temporal variations }\end{array}$ \\
\hline $\begin{array}{l}\text { Sample- } \\
\text { size }\end{array}$ & $2-\mathrm{s}(\mathrm{n})$ & 95 & (3,4) at $\mathrm{PED}$ & 2h ahead PED & $\begin{array}{l}\text { Used for testing the models sensitivity to } \\
\text { the size of the training samples }\end{array}$ \\
\hline
\end{tabular}


$1.104 \mathrm{~km}^{2}$, in 2012. The northeast of HK is mostly rural. There are 7,200,000 residents in the area of $1104 \mathrm{~km}^{2}$. Therefore, HK is one of the most densely populated regions in the world. Most of population is on the two sides of Victoria and New Territories [100].

The service sector contributes most to HK economy, which mainly includes trade, financial service, tourism, retail, etc. Industry is also an important sector in HK but mainly geared to the re-export of goods in mainland China. Due to the limitation of land and large population, heavy industries, which are land-intensive, are rare. There is no mining industry due to the lack of mineral sources. The main industries located in HK are small-size establishments, of which garment production has been leading the manufacturing sector for decades, followed by light and consumer goods such as footwear, toy, and plastics. In HK, three industrial estate areas are located at three places, namely Yuen Long, Tai Po and Tseung Kwan O (Figure 2-4). Tai Po estate area was set up in 1978. There are many manufacture establishments. Yuen Long industrial estates were built at 1983 after Tai Po. The industries at Yuen Long mainly include Petrochemical, steel production, and non-ferrous metal production. Tseung Kwan O industrial estate was found in 1995. Different from other two industrial estates, most of the industries in Tseung Kwan are high-tech.

In order to safeguard the health and well-being of the community and to build HK as a global and green city providing a high quality of life, the HK government implemented a broad range of measures to control local emissions from motor vehicles, shipping companies, power plants, and industrial and commercial processes. Moreover, since HK is affected by the regional pollution arising from various sources in the Guangdong province, a departmental collaboration was arranged between the governments of HK and Guangdong province to control pollution as introduced. The government established a policy that the daily average ozone level should not exceed $160 \mathrm{\mu g} / \mathrm{m}^{3}$ more than nine times per year. In addition, the regulation of limiting sulphur content from industrial fuel was introduced in 1990, and some programs were initiated to control the vehicle emissions in 2000, for example, adopt tighter fuel and vehicle emissions and cleaner alternatives to diesel vehicles etc. Meanwhile, the Air 
Pollution Control Ordinance and its subsidiary regulations were also launched by HK government for controlling emissions from power plants, industrial sources, and construction activities, etc.

The HK air pollution monitoring network contains 15 fixed monitoring stations: 12 general stations and 3 roadside stations as shown in Figure 2-4. The abbreviations of the stations can be found in Table 2.3 . The hourly records of carbon monoxide $(\mathrm{CO})$, fine suspended particulates (FSP), nitrogen dioxide (NO), nitrogen oxide $\left(\mathrm{NO}_{x}\right)$, ozone $\left(\mathrm{O}_{3}\right)$, respirable suspended particulates (RSP), and sulphur dioxide $\left(\mathrm{SO}_{2}\right)$ are provided by the Environmental Protection Department (EPD) of HK. The data can be downloaded free of charge from the EPD official Website [101]. The HK Observatory (HKO) offers hourly meteorological data from 42 meteorological stations, such as temperature (TMP), relative humidity (RH), and wind direction (WDR). The meteorological data can be found at the Website of the HKO [102].

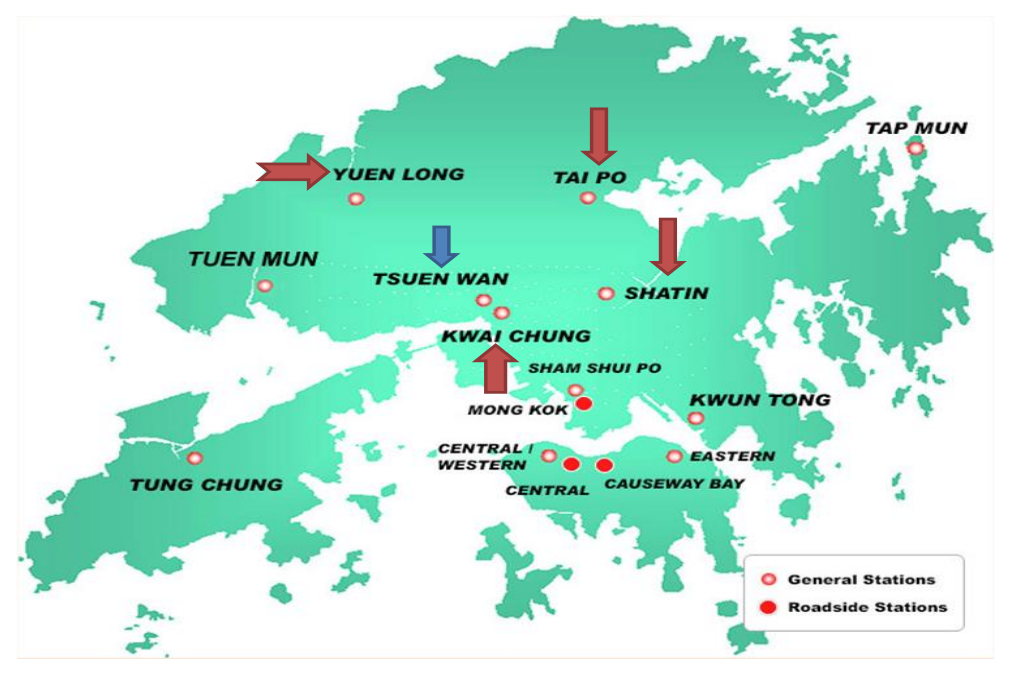

Figure 2-4: Air quality monitoring network of HK.

Data regarding manufacturer, construction, and population, and household at district level can be accessed from the website of the Census and Statistics Department of HK government across years 1999 to 2013. The traffic flow data by road was provided from years 2001 to 2007 by the Transport Department of Hong Kong 
Table 2.3: Abbreviations of the air quality monitoring stations of HK.

\begin{tabular}{l|llllllll}
\hline Stations & Central & Eastern & Kwun & Sham & Kwai & Tsuen & Yuen & Mong \\
& Western & & Tong & Shui Po & Chung & Wan & Long & Kok \\
Abbr. & CW & Eastern & KT & SSP & KC & TW & YL & MK \\
\hline Stations & Tuen & Tung & Tai Po & Sha Tin & Tap & Causeway Central & \\
& Mun & Chung & & & Mun & Bay & \\
Abbr. & Tmun & TC & TP & ST & TM & Causeway Central & \\
\hline
\end{tabular}

Government website. All the data were selected from survey-based annual reports.

\section{Target stations selection}

Target station selection server the purpose of selecting factor from neighborhood as region inputs and showing whether regional ozone transportation is an important factor involved in ozone formation empirically. Two data sets were prepared for analysis: one contained the pollution and meteorological variables only from the TW station; to the second, the variables from the other stations were added. We call the former data set the single-site data set and the latter the distance-based data set.

TW was chosen as the target station for tracking the imbalance class problem, which is an area with a moderate number of $O_{3}$ exceedances as compared to other areas in HK. The candidate stations for the distance-based data set were selected based on three aspects: data availability, distance, and their direction with respect to TW (Figure 2-5).

Since AI techniques are limited because of the limited observations, for this study a sufficient number of observations for training the AI model was needed. Thus, it was required that the ozone data at the candidate stations cover at least the same period as those for the TW station in order to obtain maximum data samples after data merging. The available $O_{3}$ records were from 1999 to 2013. Then, the MK, Central, and Causeway stations, for which ozone records exist for only two or three years, were excluded from the list of candidate stations. From the distance prospective, the 
stations that are near TW had priority for selection. Some very distant stations, such as Tmum, Eastern, and TC, were excluded. Moreover, in this study we preferred to cover selected stations located in at least four different directions from TW. The $\mathrm{KC}$, SSP, CW, and KT stations are located to the southeast of TW. KC is the closest station to TW. Then, the SSP, CW, and KT stations were excluded from the candidate stations. Therefore, they were not considered as candidate stations for the distance-based data set. Finally, ozone concentration levels from the KC, YL, TP, and ST stations were selected as the additional inputs to the distance-based data set.

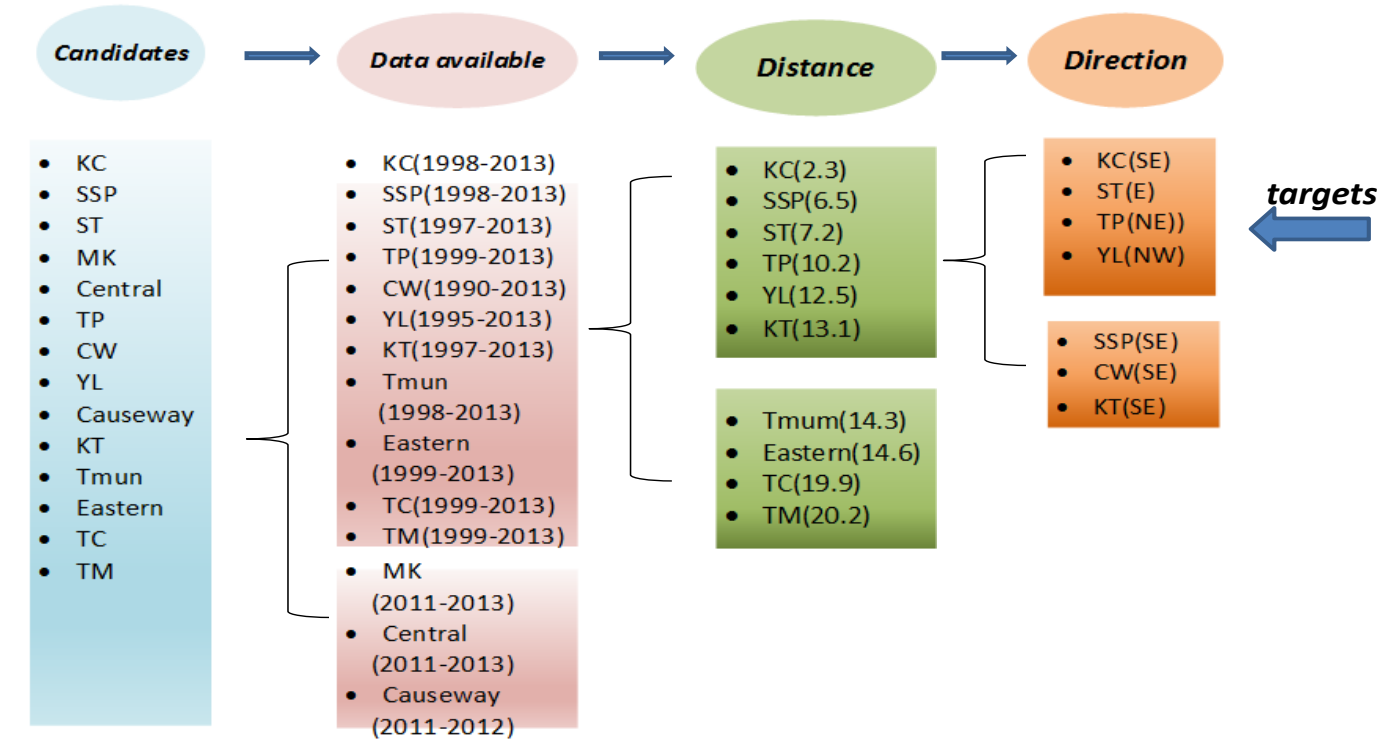

Figure 2-5: Candidate monitoring station selection for distance-based data set.

\section{Data preparation}

The variables for the daily maximum or minimum levels of ozone precursors, including $\mathrm{O}_{3}, \mathrm{NO}_{x}$, and $\mathrm{NO}_{2}$ from the previous day, the ratio of $\mathrm{NO}_{x} / \mathrm{NO}_{2}$ and meteorological factors, such as temperature $(T M P)$, relative humidity $(R H)$, wind speed $(W S P)$, 
and wind direction $(W D R)$, as well as description variables, such as season $(S)$, month $(M)$, and weekday $(W)$ were considered as candidate inputs.

To provide a fair comparison, in this study the distance-based data set was prepared first. After merging all the variables listed in Table 2.4, a total of 2149 samples was obtained in the distance-based data set. Then, the input features from only the TW station were extracted from the distance-based data set to generate the singlesite data set (Table 2.5). Finally, the single-site data set consists of 18 inputs and the distance-based set contains 22 input variables.

Eighty percent of the samples in both data sets were used for training and validation and $20 \%$ for testing. There are two types of output, one for regression mode, which is continuous values, and the second for classification mode with categorical variables. Regarding the classification, the output is labeled " $\mathrm{H}$ " or "L." "H" means the ozone concentration is higher than the limitation $160 \mu \mathrm{g} / \mathrm{m}_{3}$; otherwise it is labeled "L." Finally, 1719 samples were obtained for training and validation, 1681 of which were "L" and 38 were "H." Meanwhile, 430 samples were used for testing with 12 samples "H" and 418 "L."

The new variables were normalized by Equation 5.1 .

\subsubsection{Madrid}

Madrid is the capital of Spain and the largest municipality of the Community of Madrid. The population of metropolitan area is approximately 6.5 millions of inhabitants and the population of the city is approximately 3.2 million. The altitude of the city is $667 \mathrm{~m}$ above the sea level. Madrid experiences a cold semi-arid climate with cool winters and hot summers. Therefore, the level for some pollution like ozone, which is highly correlated with temperature factor, is much higher than the one in Winter as illustrated in Figure 2-6.

Pollution and meteorological data: Comunidad de Madrid and Agencia Estatal de Meteorologa (AEMET) are the two sources OF pollution and meteorological data. The former is consisted of monitoring stations, which can retrieve both pollution and meteorological data, whereas the latter one is available only for meteorological 
Table 2.4: Description of input variables.

\begin{tabular}{|c|c|c|c|}
\hline Categories & Input variables & Unit & Notation \\
\hline \multirow[t]{13}{*}{ Pollutants } & Maximum ozone level of the previous day at TW station & $\mu g / m^{3}$ & $\operatorname{Max}\left(\mathrm{O}_{3}\right)_{p}$ \\
\hline & Maximum nitrogen oxide level of the previous day at TW station & $\mu g / m^{3}$ & $\operatorname{Max}\left(N O_{x}\right)_{p}$ \\
\hline & Maximum nitrogen dioxide level of the previous day at TW sta- & $\mu g / m^{3}$ & $\operatorname{Max}\left(\mathrm{NO}_{2}\right)_{p}$ \\
\hline & tion & & \\
\hline & Minimum nitrogen oxide level of the previous day at TW station & $\mu g / m^{3}$ & $\operatorname{Min}\left(N O_{x}\right)_{p}$ \\
\hline & Minimum nitrogen dioxide level of the previous day & $\mu g / m^{3}$ & $\operatorname{Min}\left(\mathrm{NO}_{2}\right)_{p}$ \\
\hline & Ratio of maximum nitrogen oxide to maximum nitrogen & - & $\operatorname{Max}\left(N O_{x}\right)_{p} / \operatorname{Max}(\Lambda$ \\
\hline & dioxide level of the previous day at TW station & & \\
\hline & Maximum ozone level of the previous day at $\mathrm{KC}$ station & $\mu g / m^{3}$ & $\operatorname{Max}\left(\mathrm{O}_{3}\right)_{p-} K C$ \\
\hline & Maximum ozone level of the previous day at YL station & $\mu g / m^{3}$ & $\operatorname{Max}\left(\mathrm{O}_{3}\right)_{p_{-}} Y L$ \\
\hline & Maximum ozone level of the previous day at TC station & $\mu g / m^{3}$ & $\operatorname{Max}\left(\mathrm{O}_{3}\right)_{p-} T P$ \\
\hline & Maximum ozone level of the previous day at TC station & $\mu g / m^{3}$ & $\operatorname{Max}\left(\mathrm{O}_{3}\right)_{p_{-}} S T$ \\
\hline & New variables created by the equation in the Note & $\mu g / m^{3}$ & $\operatorname{Max}\left(\mathrm{O}_{3}\right)_{p-}$ distance \\
\hline \multirow[t]{8}{*}{ Meteorology } & Maximum temperature of the previous day at TW station & ${ }^{\circ} \mathrm{C}$ & $\operatorname{Max}(T M P)_{p}$ \\
\hline & Maximum relative humidity of the previous day at TW station & $\%$ & $\operatorname{Max}(R H)_{p}$ \\
\hline & Maximum wind speed of the previous day at TW station & $\mathrm{m} / \mathrm{s}$ & $\operatorname{Max}(W S P)_{p}$ \\
\hline & Maximum wind direction of the previous day at TW station & degree & $\operatorname{Max}(W D R)_{p}$ \\
\hline & Minimum temperature of the previous day at TW station & ${ }^{\circ} \mathrm{C}$ & $\operatorname{Min}(T M P)_{p}$ \\
\hline & Minimum relative humidity of the previous day at TW station & $\%$ & $\operatorname{Min}(R H)_{p}$ \\
\hline & Minimum wind speed of the previous day at TW station & $\mathrm{m} / \mathrm{s}$ & $\operatorname{Min}(W S P)_{p}$ \\
\hline & Minimum wind direction of the previous day at TW station & degree & $\operatorname{Min}(W D R)_{p}$ \\
\hline \multirow[t]{3}{*}{ Descriptive } & Month of current day & - & $M$ \\
\hline & Weekday of current day & - & $W$ \\
\hline & Season of current day & - & $S$ \\
\hline
\end{tabular}

Note: The variable $\operatorname{Max}\left(\mathrm{O}_{3}\right)_{p}$ distance is calculated by the following equation:

$\operatorname{Max}\left(\mathrm{O}_{3}\right)_{p-}$ distance $=\operatorname{Max}\left(\mathrm{O}_{3}\right)_{p-} \mathrm{KC} / 2.3+\operatorname{Max}\left(\mathrm{O}_{3}\right)_{p_{-}} Y L / 12.5+\operatorname{Max}\left(\mathrm{O}_{3}\right)_{p-} \mathrm{ST} / 7.2+\operatorname{Max}\left(\mathrm{O}_{3}\right)_{p-} \mathrm{TP} / 10.2$

2.3, 12.5, 7.2, and 10.2 are the distances $(\mathrm{km})$ from the corresponding stations to TW 
Table 2.5: Input and output variables of single-site and distance-based data sets.

\section{Data set Input variable}

Output

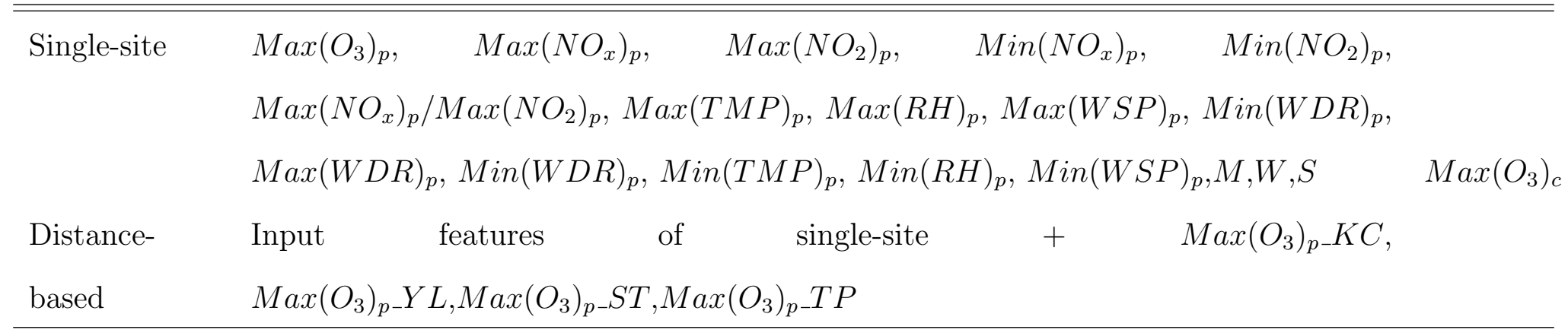
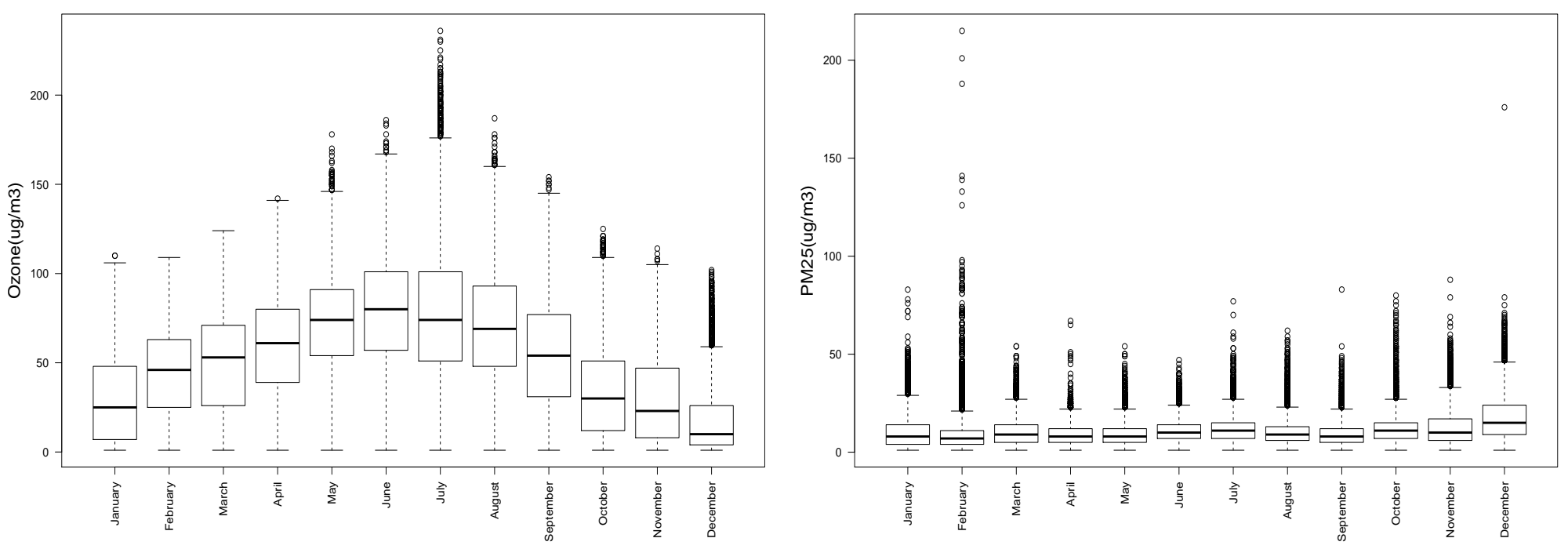

Figure 2-6: $O_{3}$ and $P M_{2.5}$ levels distribution from 2014 to 2015 
data. The pollution data can be obtained from the website of Comunidad de Madrid. The geo-locations data of air pollution and meteorological monitoring stations can be assessed from the website aforementioned as well and is shown in Figure 2-7 There are 25 air pollution monitoring stations distributed in Madrid. All the data were recorded hourly and collected from 2014 to 2015 . The days that the number of hourly pollutant recorded less than 20 hours were removed.

Individual tracking data: Since this research is the conceptual and framework exploration on health evaluation personalization of pollution exposure, the personal track data is selected from a middle-aged male professor and a young female Ph.D. student who both commute to work largely with fixed-rout and fixed modes of transport.The individual tracking data were collected by using personal Android smartphones tracking services provided by Moves application, which is available and free downloaded by both Android and IOS users(http://www.apple.com/itunes/ download/). This application is a privacy-control application, that is, if it is enabled, its users are allowed to download and extract individual location data through their smartphones. Moves can provide the address of home and work, the locations of activities, the means of commute, and the typical commute time spent each way. Finally, the space-time data provided by GPS can be extracted automatically from both individuals into JSON format as illustrated in Figure 2-8, which is a lightweight text-based data-interchange language and easy to read.

\section{Indoor pollution data selection:}

The indoor pollution data is selected from the smart sensors named Foobot as shown in Figure 2-9. This sensor can record particular matter, volatile compounds, carbon dioxide, and some meteorological variables such as temperature and humidity with interval times in seconds. The application developed by the company can be downloaded by the users in smart phone from both App store(for IOS) or Google Play Store(for Android) as illustrated in Figure 2-10. Then, through the connection between this application and smart sensor. The real time pollution and meteorological records of the the specific user, then, can be sent to the cloud sever of the company. The company provides application programming interface(API) for obtaining 


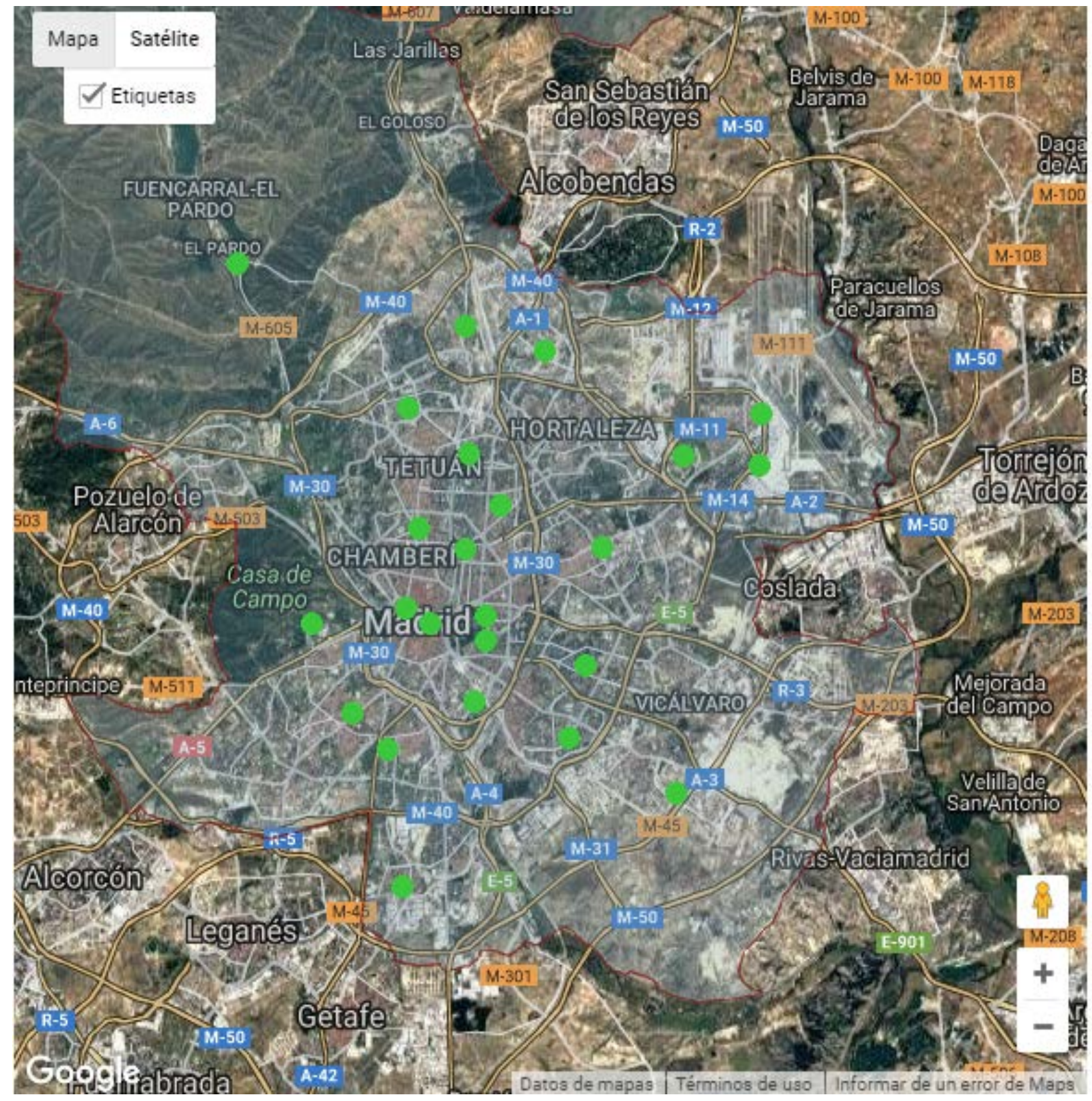

Figure 2-7: Air quality monitoring network of Madrid

Source: http://www.mambiente.munimadrid.es/sica/scripts/index.php 


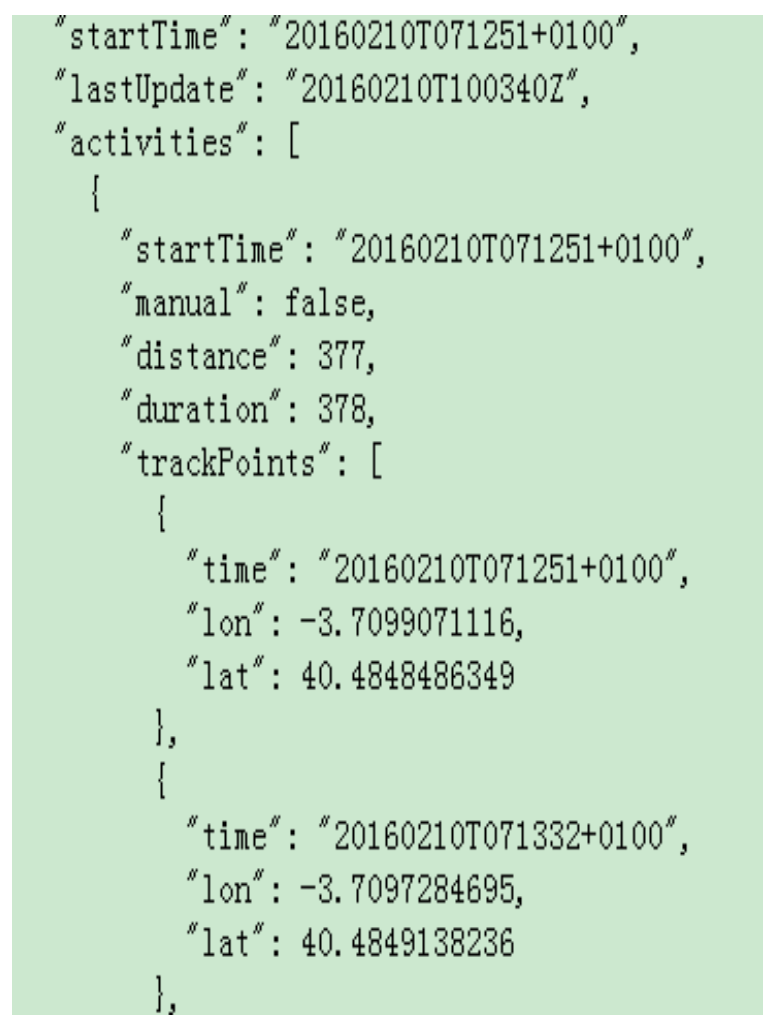

Figure 2-8: Individual tracking data in json file

the data. Finally, in order to ensure that the indoor pollution and meteorological data for the specific user is from the nearest smart sensor, the beacons in Figure 2-11 are used for identifying the nearest smart sensor that the user links to.

\subsubsection{China}

This study will focus on 30 provinces in China. Figure 2-12 shows the location of these 30 provinces. Table 6.4 illustrates the names of the provinces in China and their corresponding abbreviates, of which BJ, SH, TJ and CQ are the four most prominent metropolitans with population exceed ten million. This study takes these four cities as provincial level as they have provincial status in China based on Chinese political and administrative system. All the data are obtained from the Chinese yearbook, including energy usage for each type and each province, GDP, urbanization for each province. This study focuses on the ninth, tenth and eleventh Five Years Plan (FYP), which are from 1996 to 2000, 2001 to 2005, 2006 to 2010 respectively. 


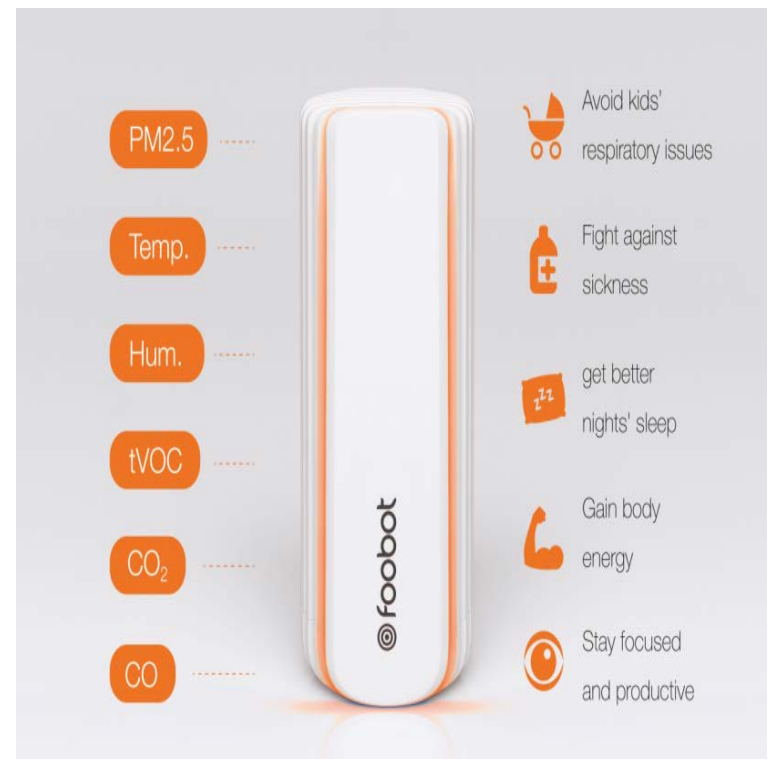

Figure 2-9: Smart sensor for indoor pollution.

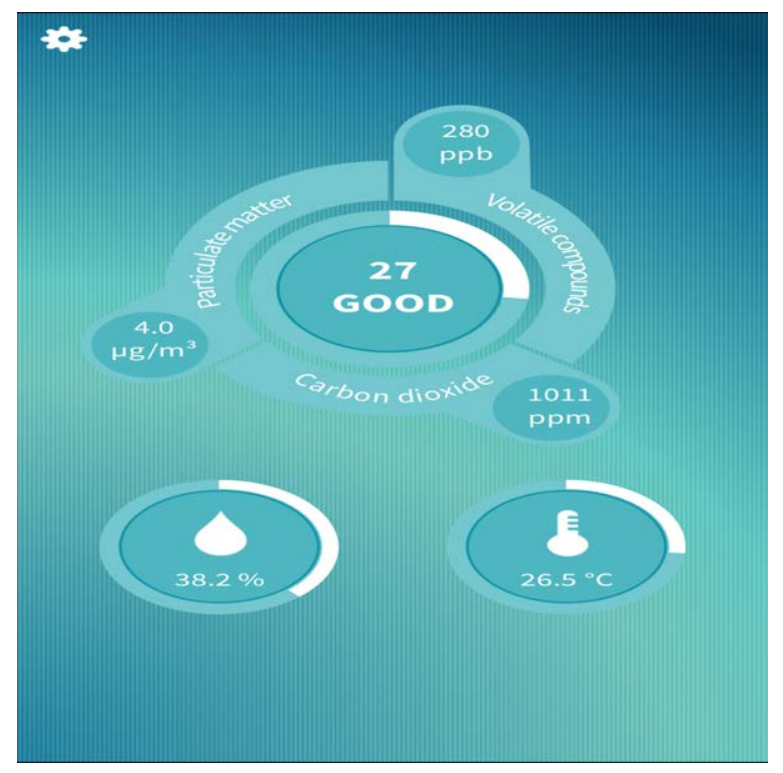

Figure 2-10: Application for Foobot. 


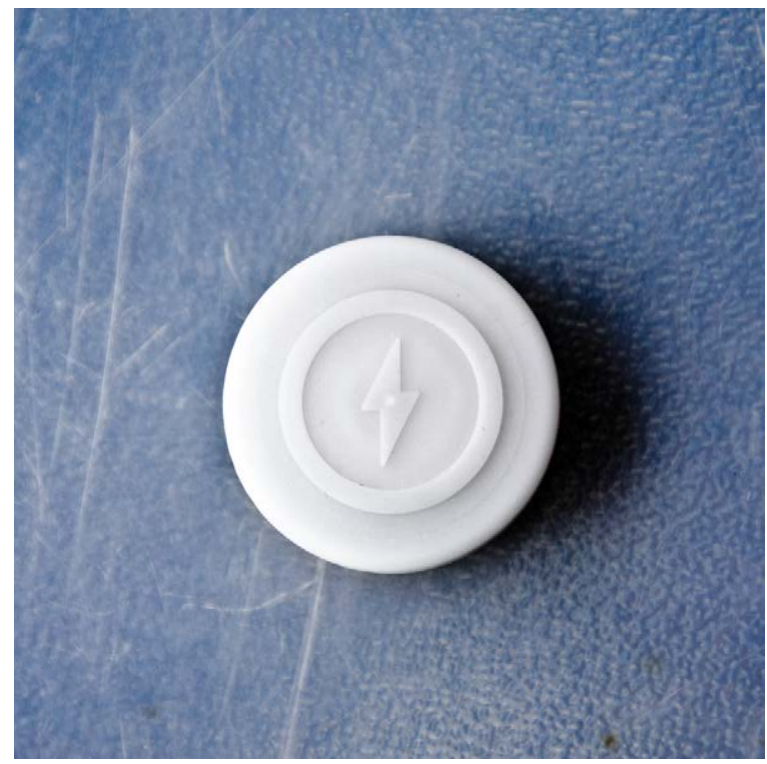

Figure 2-11: Beacons for identifying the nearest smart sensor.

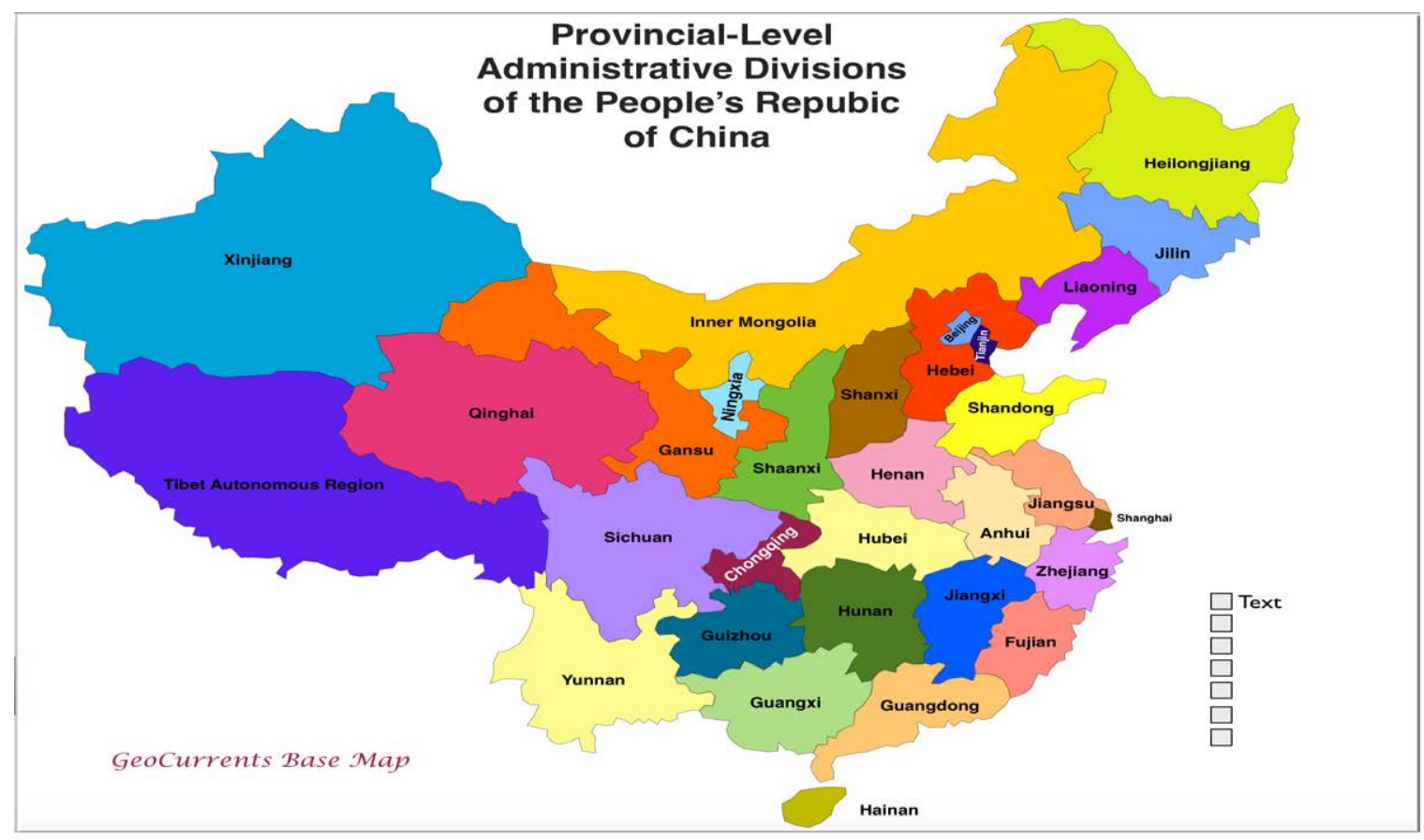

Figure 2-12: The map of provincial level administrative divisions of China. 
Table 2.6: The names of provinces and their abbreviate

\begin{tabular}{llll}
\hline Areas & Abbre & Areas & Abbre \\
\hline Bei Jing & BJ & He Nan & HN \\
Tian Jin & TJ & Hu Bei & HuB \\
He Bei & HeB & Hu Nan & HuN \\
Shan Xi & ShanX & Guang Dong & GD \\
Inner Mongolia & IM & Guang Xi & GX \\
Liao Ning & LN & Hai Nan & HaiN \\
Ji Lin & JL & Chong Qing & CQ \\
Hei Long Jiang & HLJ & Si Chuan & SC \\
ShangHai & SH & Gui Zhou & GZ \\
Jiang Su & JS & Yu Nan & YN \\
Zhe Jiang & ZJ & ShannXi & ShannX \\
An Hui & AH & GanSu & GS \\
Fu Jian & FJ & QingHai & QH \\
Jiang Xi & JX & NingXia & NX \\
Shan Dong & XinJiang & & XJ \\
\hline
\end{tabular}

\subsubsection{Industry}

The focus regarding energy used awareness is based on aN industry case. The data including the manufacturing and energy from IoT were collected from an advanced high-precision manufacturing factory located in Getafe, Spain.

The case study was established as a pilot project for the company that enabled the latter to gain a better understanding of the benefits of their particular configuration. Therefore, all the required components of the information system were provided, in addition to those in use at the company. Because of its exploratory nature, only a limited area of the production facility was selected to implement the pilot project. The duration of the use of the site works was limited to four months, including setups, 
Table 2.7: Details of an order

\begin{tabular}{lllllll}
\hline Order & Quantity & Product specifications & Machine & Kick & off & Due time \\
& & & & time & \\
\hline N1474 & 240 & Nut bearing adjust & M237 & $16 / 06 / 2014$ & $25 / 06 / 2014$ \\
& 602 & Item related to drawing: & M220 & & \\
& & 8TTAAB001808K & & & & \\
\hline \multirow{2}{*}{ N1529 } & 114 & Item related to drawing 1000173 & M237 & $12 / 06 / 2014$ & $10 / 07 / 2014$ \\
& 172 & Part Number A-118951 & M204 & & \\
\hline
\end{tabular}

configurations, data collection, etc. Due to the limited scope, only the electricity for the selected machine production was taken into account. Smart meters were installed on each machine. A cloud platform with an energy monitoring frequency of $2 \mathrm{~Hz}$ was used. Neither thermal energy usage nor general services energy usage were considered.

Table 2.7 shows two orders from the target company (N1474 and N1529), that specify quantity, production, machines and due dates. Each order stipulates two types of products. Three NC-based machines with codes M237, M204 and M220 were operating on these orders and products.

By considering order N1529 as an example, two types of products, Part Number A-118951 and Item related to drawing 1000173, were included in this order. There were 172 parts operated on by Machine 204 between 10.30 and 11:20 on July 4th, 2014 as members of Part Number A-118951. There were 114 parts machined by Machine 237 between 19:51 and 22.02 on 8th July 2014 as members of Item related to drawing 1000173. In our example, the operation column in Table 2.8 shows the operation sequences of the product. Following this order of the operation process, the log of machine 204, which is the main source of production data and contains records of all of the relevant machine activities (start time operation, machine configurations, and production ID etc.), was collected automatically and stored in the logging information system. Table 2.8 shows an excerpt of process logging for machine 204 that has been filtered from the main repository. Meanwhile, the energy data during that time were 
Table 2.8: The process log of machine 204 for Part Number A-118951

\begin{tabular}{lllll}
\hline Start time & Machine & Product & Operation Operation parame- \\
& number & ID & \multicolumn{2}{l}{ ters } \\
\hline 2014-07-04T11:00:49.027 & $\mathrm{MC}=204$ & 30 & Load & \\
2014-07-04T11:00:51.089 & $\mathrm{MC}=204$ & 30 & Face & $\mathrm{S}=1$ \\
2014-07-04T11:01:09.757 & $\mathrm{MC}=204$ & 30 & Turn & $\mathrm{D}=80, \mathrm{~S}=1, \mathrm{M}=1$ \\
2014-07-04T11:01:30.377 & $\mathrm{MC}=204$ & 30 & Chamfer & $50, \mathrm{R}=3, \mathrm{~S}=1$ \\
2014-07-04T11:01:36.284 & $\mathrm{MC}=204$ & 30 & Turn & $\mathrm{D}=58.5, \mathrm{~S}=2, \mathrm{M}=1.5$ \\
2014-07-04T11:01:44.961 & $\mathrm{MC}=204$ & 30 & Chamfer & $45, \mathrm{R}=1.3, \mathrm{~S}=1$ \\
2014-07-04T11:01:48.460 & $\mathrm{MC}=204$ & 30 & Thread & $\mathrm{S}=2$ \\
2014-07-04T11:01:51.680 & $\mathrm{MC}=204$ & 30 & Remove & $\mathrm{S}=1$ \\
2014-07-04T11:01:53.838 & $\mathrm{MC}=204$ & 30 & Unload & \\
\hline
\end{tabular}

Table 2.9: The process log of machine 204 for Part Number A-118951

\begin{tabular}{lllllllllllll}
\hline Hour & 1 & 2 & 3 & 4 & 5 & 6 & 7 & 8 & 9 & 10 & 11 & 1 \\
\hline EUR/MWH18.7 & 114.36 & 105.08 & 101.93 & 94.95 & 96.16 & 109.43 & 108.57 & 114.18 & 118.23 & 120.54 & 1 \\
\hline Hour 13 & 14 & 15 & 16 & 17 & 18 & 19 & 20 & 21 & 22 & 23 & 2 \\
EUR/MWH20.54 & 118.6 & 117.83 & 115.13 & 114.77 & 118.03 & 120.31 & 120.62 & 123.72 & 123.94 & 118.84 & 5 \\
\hline
\end{tabular}

collected by the smart meters.

The energy data can be merged with some other data stream by using the time stamp. In order to obtain information about the cost of energy used and $\mathrm{CO}_{2}$ emissions, it is necessary to know the types of energy, the historical prices of the energy and the $\mathrm{CO}_{2}$ emission factors for each type of energy. The prices of electricity in Spain, where the target company is located, can be found on the OMIE [103] website. The prices were recorded hourly. Table 2.9 shows the electricity prices on July 4th, 2014. The $\mathrm{CO}_{2}$ emissions can be derived from the equations in Table 2.1.5. The $\mathrm{CO}_{2}$ emission for electricity is 0.372 in Spain 104].

Table 2.1.5 provides equations to illustrate how to calculate the energy used, 
energy costs and $\mathrm{CO}_{2}$ emitted per operation, per product and per order. Based on it, Finally, the machine data, energy data, energy price and $\mathrm{CO}_{2}$ emission can be fully integrated and analyzed, as is partly shown in Table 2.11

\section{$2.2 \quad$ Methodology}

This section will introduce various of traditional and advanced methodologies that need to be employed for all the case studies.

\subsubsection{Feature selection and reduction}

Feature extraction and feature selection are the two main methods to reduce the dimensionality of the features that have been getting attention from researchers. The former one is related to constructing new features based on the initial feature space, which is always independent of the AI technique. PCA and factor analysis are the most popular techniques in the pollution forecasting domain[105, 106, 97, 107]. The results from [107, 108] have shown that the combination of multiple regression and ANN with component analysis not only eliminates data collinearity and reduces the models complexity, but also improve the ozone prediction accuracy. The latter one, on the other hand, involves selecting a subset of the features from the existing candidate features based on an objective function. In light of their different search strategies, the feature selection methods can be classified into three groups, namely filters, wrappers and embedded [109, 110, 111, 57]. The filter method is totally independent of the AI algorithm. It uses simple statistical methods to select the optimal subset of features, those which pass some criterion. Wrapper methods, on the other hand, need to cooperate with the AI algorithm. The whole process of feature selection occurs during the AI training. Recursive Feature Elimination and Simulated Annealing are two types of wrappers that are used widely and successfully in many applications $[112$, 113. 114]. In this study, PCA, RFE and SA algorithms are employed for feature selection. The pseudocodes for RFE and SA are described in the appendices. 
Table 2.10: Equations illustrating how the types of multi-level energy awareness can be calculated.

\begin{tabular}{|c|c|c|}
\hline & Equation & Explanation \\
\hline 1 & $E_{o p}(M ; I D ; j)=\int_{T_{s t a r t}\left(M_{i} ; I D ; i\right)}^{T_{\text {end }}\left(M_{i} ; I D ; i\right)} E(M, t) . d t$ & $\begin{array}{l}\text { Calculate the energy used in processing operation ID, instance } j \text {, at machine } M \text {, where } \\
E_{o p} \text { means Energy consumed per operation and is determined by considering the mea- } \\
\text { sured energy at machine } M(E(M, .)) \text { between the relevant times } T \operatorname{start}(M ; I D ; j) \\
\text { and } \operatorname{Tend}(M ; I D ; j) \text { as obtained from the machine controller. }\end{array}$ \\
\hline 2 & $\begin{array}{l}E p r d(I D ; j)=\sum_{i=1}^{N} \sum_{k=1} K_{I D}^{i} E_{o p}\left(M_{i} ; I D_{k} ; j\right)+ \\
\int_{T_{\text {start }}(I D ; j)}^{T_{\text {end }}(I D ; j)} \lambda(t) \cdot \sum_{s=1}^{S} E_{g}(s, t) \cdot d t\end{array}$ & $\begin{array}{l}\text { Calculate the energy used to produce product ID, instance } j(\operatorname{Epr} d(I D ; j)) \text { by includ- } \\
\text { ing all operations } I D_{k} \text { required at machine } M_{i}\left(K_{I D}^{i}\right) \text {. This is done by considering all } \\
\text { the machines in the factory }(N) \text { as well as the part of the common factory services } \\
\text { involved }(E g(s) s \in(1,, S) \text {, where } S \text { is the total auxiliary services linked to production } \\
\text { that the factory provides, according to its specific participation }(\lambda: l \text { over the total } \\
\text { number of products being produced at that particular time). }\end{array}$ \\
\hline 3 & $\begin{array}{l}E_{\text {ord }}(O I D)=\sum_{q=1}^{Q_{p r d}} \sum_{p r d=1}^{p} E_{p r d}\left(I D_{p r d} ; q\right)+ \\
\sum_{m=1}^{M} \int_{T_{u p-s t a r t}(M ; I D ; j ; j)}^{\left.T_{u p p s}\right)} E(m, t) . d t \\
\int_{T_{\text {not }}(O I D)} \lambda(t) \cdot \sum_{s=1}^{S} E_{g}(s, t) . d t\end{array}$ & $\begin{array}{l}\text { Calculate the energy used to produce order ID }\left(E_{\text {ord }}(O I D)\right) \text {, which involves the sum- } \\
\text { mation of the per product energy consumption through all the instances of the re- } \\
\text { quired products }\left(Q_{p r d}\right) \text {. It also includes the energy demand per machine setup, as well } \\
\text { as the general services consumption not specifically linked to production of products } \\
\left(T_{n o t}(O I D) \subset\left(\left[T_{s t a r t}(O I D), T_{e n d} O I D\right)\right]-\cup\left[T_{\text {start }}\left(I D_{p r d} ; q\right), T_{\text {end }}\left(I D_{p r d} ; q\right)\right]\right) \text {, where } \\
\text { the } O I D \text { includes } Q_{O I D} \text { number of instances. Every product instance is nominated } \\
\text { as } I D_{p r d} \text {. }\end{array}$ \\
\hline
\end{tabular}

$4 C E_{o p}(o ; M ; I D ; j)=\int_{T_{s t a r t}\left(M_{i} ; I D ; i\right)}^{\left.T_{e n} M_{i} I I ; i\right)} C_{\text {ener }}(o ; t) . E(o, M, t) . d t$ alculate the cost of energy used to produce an operation ID, instance $j$, at machine $M$, which depends on the selected energy source $(o)$, as well as its specific cost during the relevant period of time $\left(T_{\text {start }}(M ; I D ; j), T_{\text {end }}(M, I D, j)\right)$. Note that it is now required to know the source of the energy being consumed at machine $M(E(o, M, t))$ to ensure precise costs.

$5 \quad C E \operatorname{Erd}(I D ; j)=\sum_{i=1}^{N} \sum_{k=1} K_{I D} \sum_{l=1}^{O} C E_{o p}\left(o_{l} ; M_{i} ; I D_{k}\right.$ Cjej)leulate the cost of energy used to produce a product $I D$, instance $j\left(C E_{p r d}(I D, j)\right)$ $\int_{T s t a r t(I D ; j)}^{T e n d(D ; j)} \lambda(t) \cdot \sum_{s=1}^{S} \sum_{l=1}^{O} C_{\text {ener }}(l, t) \cdot E_{g}(l, s, t) \cdot d t \quad$ by including all the operations $I D_{k}$ required to develop the instance $j$ of product $I D\left(K_{I D}\right)$ over the machines $M_{i}$ involved, as well as the part of the common factory services directly related to production involved. Here, several potential energy sources have been considered $\left(o_{l}, l=1, O\right)$.

$6 C E_{\text {ord }}(O I D)=\sum_{q=1}^{Q_{p r d}} \sum_{p r d=1}^{p} C E_{p r d}\left(I D_{p r d} ; q\right)+$ Calculate the cost of energy used to produce an order $O I D\left(C E_{\text {ord }}(O I D)\right)$, which $\sum_{m=1}^{M} \int_{T_{u p-s t a r t}(M ; I D ; j)}^{T_{u p-s a r t}(M ; D ; j)} \sum_{l=1}^{O} C_{\text {ener }}(l ; t) \cdot E(l, m, t) . d t+$ involves the summation of the cost per product $\left(I D_{p r d}\right)$ energy consumption, includ$\int_{T_{\text {not }}(O I D)} \lambda(t) \cdot \sum_{s=1}^{S} \sum_{l=1}^{O} C_{\text {ener }}(l, t) \cdot E_{g}(s, t) \cdot d t$ ing all the required product instances $\left(Q_{p r d}\right)$ plus the energy demand per machine setup according to the source of energy involved $(1,, O)$. It is required to include the consumption of general services that are not specifically linked to production of products.

$7 \quad C O_{2 o p}(o ; M ; I D ; j)$

$\int_{T s t a r t\left(M_{i} ; I D ; i\right)}^{T e n d\left(M_{i} ; D ; i\right.} F_{\text {ener }}(o ; t) . E(o, M, t) \cdot d t$

$=$ Calculate $\mathrm{CO}_{2}$ emissions that result from processing an operation $I D$, instance $j$, at machine $M$, which depends on the origin of such an energy source, as well as its specific $\mathrm{CO}_{2}$ emission factor $F_{\text {ener }}(o,$.$) .$

$8 \quad C E \operatorname{prd}(I D ; j)=\sum_{i=1}^{N} \sum_{k=1} K_{I D} \sum_{l=1}^{O} C O 2_{o p}\left(o_{l} ; M_{i} ; I D D_{k} ; \mathrm{a} d \dot{\gamma} ;\right.$ thlate $\mathrm{CO}_{2}$ emissions that result from producing a product $I D$, instance $j$, by $\int_{T s t a r t(I D ; j)}^{T e n d(I ; j)} \lambda(t) \cdot \sum_{s=1}^{S} \sum_{l=1}^{O} F_{\text {ener }}(l, t) \cdot E_{g}(l, s, t) \cdot d t \quad$ including all of the operations $I D_{k}$ involved by the instance $(j)$ over the machines $M_{i}$, as well as the part of the common factory services directly related to the production involved.

$9 C E_{\text {ord }}(O I D)=\sum_{q=1}^{Q_{p r d}} \sum_{p r d=1}^{p} C o 2_{p r d}\left(I D_{p r d} ; q\right)+$ Calculate $\mathrm{CO}_{2}$ emissions that result from producing an order ID, which involves the $\sum_{m=1}^{M} \int_{T_{u p-s t a r t}(M ; I D ; j)}^{T_{u p-s t a r}(M ; D)} \sum_{l=1}^{O} C_{\text {ener }}(l ; t) . E(l, m, t) . d t+$ summation of the per product energy emission plus the emissions per machine setup, $\int_{T_{\text {not }}(O I D)} \lambda(t) \cdot \sum_{s=1}^{S} \sum_{l=1}^{O} F_{\text {ener }}(l, t) \cdot E_{g}(s, t) \cdot d t \quad$ as well as the consumption of general services that are not specifically linked to production of products. 
Table 2.11: Summary of merged data of process logging, energy consumption, cost of energy used and CO2 emission

\begin{tabular}{|c|c|c|c|c|c|c|c|}
\hline Start Time & $\begin{array}{l}\text { Machine } \\
\text { number }\end{array}$ & ID & Operation & $\begin{array}{l}\text { Configuration pa- } \\
\text { rameters }\end{array}$ & $\begin{array}{l}\text { Energy } \\
\text { (Wh) }\end{array}$ & $\begin{array}{l}\text { Energy } \\
\text { cost(Cents } \\
\text { of EUR) }\end{array}$ & $\mathrm{CO}_{2}(\mathrm{~g})$ \\
\hline 2014-07-04T11:00:14.666 & $\mathrm{MC}=204$ & 29 & Turn & $\mathrm{D}=80, \mathrm{~S}=1, \mathrm{M}=1$ & 17.79 & 0.105 & 6.618 \\
\hline 2014-07-04T11:00:26.176 & $\mathrm{MC}=204$ & 29 & Chamfer & $50, \mathrm{R}=3, \mathrm{~S}=1$ & 0.319 & 0.002 & 0.119 \\
\hline 2014-07-04T11:00:27.606 & $\mathrm{MC}=204$ & 29 & Turn & $\mathrm{D}=58.5, \mathrm{~S}=2, \mathrm{M}=1.5$ & 10.959 & 0.065 & 4.077 \\
\hline 2014-07-04T11:00:36.768 & $\mathrm{MC}=204$ & 29 & Chamfer & $45, \mathrm{R}=1.3, \mathrm{~S}=1$ & 4.5 & 0.027 & 1.674 \\
\hline 2014-07-04T11:00:40.724 & $\mathrm{MC}=204$ & 29 & Thread & $\mathrm{S}=2$ & 6.633 & 0.039 & 2.468 \\
\hline 2014-07-04T11:00:46.261 & $\mathrm{MC}=204$ & 29 & Remove & $S=1$ & 1.249 & 0.007 & 0.465 \\
\hline 2014-07-04T11:00:46.980 & $\mathrm{MC}=204$ & 29 & Unload & & 2.848 & 0.017 & 1.0599 \\
\hline 2014-07-04T11:00:49.027 & $\mathrm{MC}=204$ & 30 & Load & & 0.145 & 0.001 & 0.054 \\
\hline 2014-07-04T11:00:51.089 & $\mathrm{MC}=204$ & 30 & Face & $\mathrm{S}=1$ & 16.517 & 0.098 & 6.144 \\
\hline 2014-07-04T11:01:09.757 & $\mathrm{MC}=204$ & 30 & Turn & $\mathrm{D}=80, \mathrm{~S}=1, \mathrm{M}=1$ & 26.658 & 0.105 & 9.917 \\
\hline 2014-07-04T11:01:30.377 & $\mathrm{MC}=204$ & 30 & Chamfer & $50, \mathrm{R}=3, \mathrm{~S}=1$ & 6.656 & 0.002 & 2.476 \\
\hline
\end{tabular}




\section{Principle component algorithm}

Principle Component Analysis (PCA) is widely used as a method to select subsets of variables for a regression equation. It transforms the original set of variables into a new set of an equal number of independent and uncorrelated variables or principal components (PCs) [97, 107]. Most of the variation of the data set can be explained by the first few ordered principle components. Each original variable is maximally correlated with only one principal component and a near zero associate with the other components. Meanwhile, a varimax rotation matrix can be obtained that ensure each variable is maximally correlated with only one principal component and not be association with other components[107]. The new input matrix will be obtained by the following equation:

$$
X * \beta(n)=X^{*}
$$

$X$ is the original inputs; $p$ is the varimax rotation matrix after transformation; (n) stands for varimax rotation matrix with the first $n$ principle components that can explain more than $75 \%$ variances of original input variables. $X^{*}$ is the new input matrix.

\section{Simulated annealing}

Simulated Annealing (SA) is a method for searching optimal solution . It was first proposed by Kirkpatrick, S. and Gelatt, C. D. and Vecchi, M. P. [115]. SA was inspired from the process of annealing in metal work. Annealing involves heating and cooling a material to alter its physical properties due to the changes in its internal structure. As the metal cools, its new structure becomes fixed, consequently causing the metal to retain its newly obtained properties. SA creates initial solution and sets an initial temperature. If the new solution is better than the previous one, the new solution is accepted. Or if earlier solution is better than the previous one but the differences are within the acceptance probability, the worse one still can be accepted. Therefore, it allows this algorithm jump out of the local optimums, and gets a global optimums. In this study, the integrating of SA and RF (SA+RF) as well 
as $\mathrm{SA}$ and RF (SA+MLR) are applied. SA algorithm controls the subset of input variables. A solution is a binary vector that describes the current subset. The subset is perturbed by randomly changing a small number of members. Then it sends the subset to classifiers or regressors for training. In contrast to some statistic method like principle component analysis which is performed totally independently on classifiers or regressors, the process of feature selection by SA depends on the training results of classifiers or regressors. This integration algorithm is more complicated and could consume more time. Nevertheless, it could obtain optimal or near optimal subset features.

\section{Recursive Feature Elimination}

One of the wrappers methods, namely Recursive Feature Elimination, is particularly computationally advantageous and robust against over-fitting. However, compared PCA and filter selection methods, it is still computation time-consuming. Table 2.13 show the The pseudocode for Backwards Selections. For each interaction, the predictor(learner) should be reconstructed and the error of the predictor need to be re-computed. For some learners, such as ANN and SVM with tuning parameters, it will require computation than the non-parameter predictor, like CART and RF.

\subsubsection{Preprocessing for imbalanced data}

\section{Over-sampling (OS)}

The mechanics of over-sampling consists of appending a set of samples by replicating the selected examples from the minority class. Then, the original distribution of balance is adjusted. However, owing to the replication of samples, the likelihood of over-fitting increases when classifiers produce multiple clauses in a rule for multiple identical samples, which leads to the rule becoming too specific. Then, the forecasting accuracy is sensitive to changes in the testing data [116, 117]. 
Table 2.12: The pseudocode for the integrating of Simulated Annealing

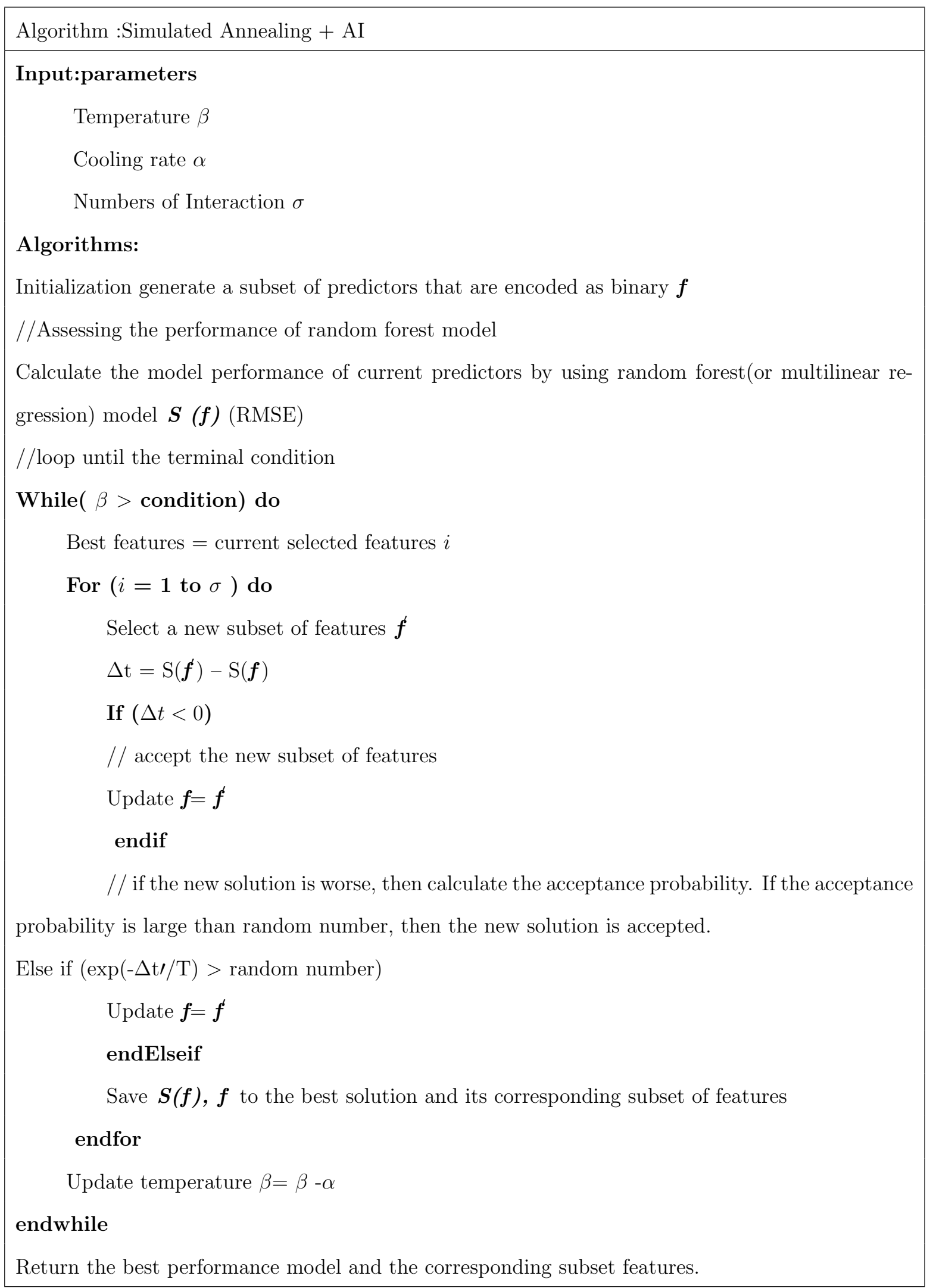


Table 2.13: The pseudocode for Recursive Feature Elimination

\begin{tabular}{l} 
Algorithm : Recursive Feature Elimination \\
\hline Input: parameters \\
All predictors $S$ \\
Learner $M$ \\
Numbers of re-sampling Interaction $\delta$ \\
Sizes of subset features $N=\left(N_{1}>N_{2}>N_{3}, \ldots, N_{s}\right)$
\end{tabular}

Algorithms:

For $(i=\mathbf{0}$ to $\delta)$ do

Training the model $M$ with all the predictors $S$

//Assessing the performance(RMSE) of the learner $M$ over features $S$

error $=R M S E_{(S)}$

compute the ranking criteria Calculate the variable importance or rankings $S$

//loop For subset size $N_{i}$

For $\left(N_{i}=\right.$ from $N_{1}$ to $\left.N_{s}\right)$ do

keep the top number of $N_{i}$ features $S_{N_{i}}$

Train the model using $S_{N_{i}}$

calculate the model performance $\mathrm{R}(\mathrm{SI})$ by predictors $S_{N_{i}}$

Re-rank the predictors $S_{N_{i}}$

endfor

endfor

//Average the performances over all the subsets of features $S_{N_{i}}$ using validation samples

Select the best subset of features $S_{j}$

Fit model corresponding to the predictors $S_{j}$ 


\section{Under-sampling (US)}

The under-sampling (US) method retains the minority instances and selects a certain percentage of majority samples to balance the two classes. The main drawback of this method is the probability that some important instances are discarded. In order to retain as many majority samples as possible and determine whether the ratio of the minority samples to that of the majority affects the modeling accuracy, the proportions of the minority samples are maintained at 10\% (US10) and 30\% (US 30) of the total samples, respectively, in the under-sampling process.

\section{Synthetic Minority Over-sampling Technique (SMOTE)}

The Synthetic Minority Over-sampling Technique (SMOTE) is a hybrid method first proposed by Chawla in 2002 [118]. It oversamples by creating "synthetic" instances rather than by means of replacement. In detail, it calculates the differences between one minority sample $(x, y)$ and its nearest neighbor $\left(x^{\prime}, y^{\prime}\right)$. The difference between these two points can be calculated: $(\Delta x, \Delta y)=\left(x-x^{\prime}, y-y^{\prime}\right)$. Meanwhile, a random number $\alpha$ between 0 and 1 is generated. Finally, the new sampled are obtained by $\left(x_{\text {new }}, y_{\text {new }}\right)=(x, y)+\alpha *(\Delta x, \Delta y)$.

SMOTE not only adjusts the sampling balance, but also prevents the overfitting problem by avoiding the copied samples from the minority class and keeps the valuable information of the original samples. In our study, five new instances were generated for each minority instance. A total of $170 \%$ of the samples of the original majority instances were selected randomly.

\section{Comparison of preprocessing}

Arguments presented in the existing studies about the strengths and weaknesses of these three state-of-the-art processing methods have been discussed in the data mining community literature [119, 69, 117]. The main disadvantage of under-sampling methods is relatively obvious, that is, removing samples from the majority class could lead to a loss of importation information for the modeling. In the case of oversampling, 
the multiple copies samples appended to the original data increase the probability of over-fitting. SMOTE is a powerful algorithm, which can avoid overfitting issues in oversampling, but it also generates a relatively balanced data set. However, it is prone to produce noise.

\subsubsection{Statistical modeling and machine learning}

In this part, the statistical models, single and ensemble machine learning algorithms will be introduced. The statistical models includes Multiple Linear Regression, time series, panel data analysis, and Kriging models. The single ML algorithm including Artificial Neuron Network, Support Vector Machine, and Classification and Regression Tree will be described. Regarding ensemble algorithms, this study prefers to employ Bagging, Boosting and Stacking. Theses three algorithms are considered to be ones of the most powerful algorithm to improve the forecasting accuracy and enhance generalization capability for single algorithm, and have been widely applied in many real-world applications.

\section{Statistical modeling}

\section{Multiple Linear Regression(MLR)}

Multiple Linear Regression(MLR) is a basic and easier linear model which make a linear combination of input variables [30, 93]. The mathematical equation is as following:

$$
y_{i}=\beta_{0}+\beta_{1} x_{i 1}+\beta_{2} x_{i 2}+\ldots+\beta_{j} x_{i j}
$$

where $y_{i}$ is the dependent values $i ; \beta_{i}$ is the regression coefficient of input variable $j ; x_{i j}$ is the $i^{t h}$ value of input variable $j$.

\section{Panel data analysis}

Panel data that is also named longitudinal or cross-section time-series data, is a dataset in which the same individuals or entities are observed over time. The advantage of panel data is that it allows to control for variables that cannot be observed. 
The industrial sector could be the main contributing source to air pollution in Hong Kong [120, 121, 122]. Since there are three industrial estates, which are the zones for industrial development. This study assumes the pollution from the industrial estate areas are higher than from other areas. Therefore, in order to control the "industry" factor affecting the output. Panel data analysis will be employed.

The most popular techniques utilized to analyze panel data should be fixed effects model and random effects model. In this study case, Industrial Area represents the entities or panel $(i)$, and "year" represents the time variable $(t)$. Then, the fix model is as Equation 2.4.

$Y_{i}(t)=\beta_{0}+\beta_{1} X_{1, i}(t)+\beta_{2} X_{2, i}(t)+\ldots+\beta_{k} X_{k, i}(t)+y_{i} E_{i}+\left(\alpha_{i} \mid E\right)+\mu_{i}(t) \operatorname{Cov}\left(\mu_{i}(t), X_{k, i}(t)\right) \neq 0$

$X_{k, i}(t)$ is the independent variable value at area $i$, time $t ; \beta_{k}$ is the coefficient; $E_{i}$ is the industrial estate area, which is binary dummy; $y_{i}$ is the coefficient for the dummy variable; $\alpha_{i}$ is the unobserved effect; and $\mu_{i}(t)$ is the error term.

The models above are built varying types of pollutants as output $Y$, which includes $\mathrm{NO}_{x}$ and $\mathrm{NO}_{2}$. For pollutants $\mathrm{SO}_{2}, \operatorname{RSP}\left(\mathrm{PM}_{10}\right)$, and $\mathrm{O}_{3}$ that are characterized by uptrend across the years, it is likely to have time-dependent variation of such pollutants and relevant to add time effects on the entity effects models. The new equation after dummies are presented as Equation 2.5.

$Y_{i}(t)=\beta_{0}+\beta_{1} X_{1, i}(t)+\beta_{2} X_{2, i}(t)+\ldots+\beta_{k} X_{k, i}(t)+y_{i} E_{i}+\sigma_{2} T_{2}+\ldots+\sigma_{t} T_{t}\left(\alpha_{i} \mid E\right)+\mu_{i}(t)$

Where $T_{t}$ is time as dummy variable and $\sigma_{2}$ is coefficient for time dummy variable.

Random-effects model, on the other hand, assumes the entitys error is uncorrelated 
with input variables. The Equation 2.6 of random effects model.

$$
Y_{i}(t)=\beta_{0}+\beta_{1} X_{1, i}(t)+\beta_{2} X_{2, i}(t)+\ldots+\beta_{k} X_{k, i}(t)+\left(\alpha_{i} \mid E\right)+\mu_{i}(t)+\varepsilon_{i}(t) \operatorname{Cov}\left(\mu_{i}(t), X_{k, i}(t)\right)=0
$$

It should be noted that $\mu_{i}(t)$ is between entity error, while $\varepsilon_{i}(t)$ is within-entity error. If the differences among entities can be determined, the random effects model should be used. Heteroskedasticity test is employed to see if the error variances are independent of any explanatory variable. Finally, Hausman test will be conducted in order to find out which of these two models is preferred. Specifically if $\mathrm{p} i \mathrm{0} 0.05$, the hypothesis that there are no significant difference between fix-effect model and random-effect model is accepted. Either of them can be selected, otherwise, the random-effect model should be chosen.

\section{Kriging}

An accurate estimation of air pollution level in the places lack of monitoring stations is the foundation for health effect evaluation. Kriging algorithm is widely used for spatial interpolation.

Kriging algorithm is also called space auto-covariance optimal interpolation method, which is named by mining engineering D.G.krige. It is a very useful tool for geological statistics grid handling and has been applied to ground water modeling, soil mapping, and many other fields. It considers the spatial properties variations in spatial position, makes sure the influential range of plugged point value, and then uses the sampling points to estimate the value of the points to be inserted. In mathematics, this method can provide a best linear unbiased estimation to find out the certain value of points, considering the shape and the size sample information, space position of geometric characteristics, and grade of space structure. It aims to achieve the linear, unbiased and minimum variance of estimation, and gives certain coefficient to each sample.

Definition of kriging: Kriging is a geostatistical term for optimal linear prediction of a spatial process, the statistical counterpart to kernel interpolation. Kriging 
is based on the modeling assumption, that is, $\mathrm{f}$ is a realization of a random field $\mathrm{Z}$, which is a collection $z(x): x \in \mathrm{T}$ of random variables over the same probability space $(\Omega, \mathrm{A}, \mathrm{P})$, indexed over $\mathrm{T}$. The observation $\mathrm{z}\left(\mathrm{x}_{0}\right) \ldots, \mathrm{z}\left(\mathrm{x}_{n}\right)$ are then realizations of the random variables $\left(\mathrm{x}_{0}\right), \ldots \mathrm{Z}\left(\mathrm{x}_{n}\right)$. To predict $z$ at some (unobserved) location $x_{o}$ $\in \mathrm{T}$, one considers all linear predictors of the form as Equation 2.7 .

The ordinary kriging can be presented as Equation 2.7

$$
\widehat{z_{0}}=\sum_{n=0}^{n} \lambda_{i} z_{i}
$$

Where $\widehat{z_{0}}$ is the estimated value at point $\left(x_{0}, y_{0}\right), z_{0}$ is the real value at point $x_{0}, y_{0} . \lambda_{i}$ are the weights. To determine optimal weights $\lambda_{i}$, additional structural assumptions on $z$ are required. According to what we use, this research only discusses the ordinary kriging.

Assumption of kriging: The assumption that $z_{o}$ is centered seems inappropriate in most applications of geostatistics. The first generalization of ordinary kriging is, therefore, to allow for a non-zero mean function $m(x):=E(z(x))$ while still keeping the assumption that $z$ has second moments. The mean function is usually unknown in practice, but this problem can be bypassed by requiring the potential predictors $z_{i}$ of $z$ to be unbiased, i.e. Equation 2.9

An assumption is that for any $(x, y)$, the expectation of value $z$ is constant $c$ and variance is $\sigma^{2}$, leading to what is called ordinary kriging. Accordingly, what is expected is to obtain a vector of weight $\lambda_{i}$ that achieve the minimum value of (Equation 2.8) by requiring the potential predictors $\widehat{z_{o}}$ of $z_{o}$ to be unbiased (Equation 2.9 .

$$
\begin{gathered}
J=\min _{\lambda_{i}} \operatorname{Var}\left(\widehat{z_{o}}-z_{o}\right) \\
\widehat{z_{o}}-z_{o}=0
\end{gathered}
$$




$$
\begin{gathered}
E(z(x, y))=E[z]=c \\
\operatorname{Var}[z(x, y)]=\sigma^{2}
\end{gathered}
$$

Therefore, the value at point $(x, y)$ is consisted of the expectation in the distance $h$ and random bias $R(x, y)$ (Equation 2.12).

$$
\begin{gathered}
z(x, y)=E[z(x, y)]+R(x, y)=c+R(x, y) \\
\operatorname{Var}[R(x, y))]=\sigma^{2}
\end{gathered}
$$

Equation 2.14, equality constraint, is generated by inferring from Equation 2.7. Equation 2.9 and Equation 2.10.

$$
\sum_{n=0}^{n} \lambda_{i}=1
$$

Optimization with equality constraints: The object function(Equation 2.8) can be written as Equation 2.15

$$
J=\operatorname{Var}\left(\widehat{z_{o}}-z_{o}\right)=\operatorname{Var}\left(\sum_{i=0}^{n} \lambda_{i} z_{i}-z_{o}\right)=\operatorname{Var}\left(\left(\sum_{i=0}^{n} \lambda_{i} z_{i}\right)-2 \operatorname{Cov}\left(\left(\sum_{n=0}^{n} \lambda_{i} z_{i}, z_{o}\right)+\operatorname{Cov}\left(z_{o}, z_{o}\right)\right.\right.
$$

Where

$$
\begin{gathered}
C_{i j}=\operatorname{Cov}\left(z_{i}, z_{j}\right)=\operatorname{Cov}\left(R_{i}, R_{j}\right) \\
R_{i}=z_{i}-c
\end{gathered}
$$

Finally, the objective function $J$ can be written as Equation 2.18.

$$
J=\sum_{n=0}^{n} \sum_{j}^{n} \lambda_{i} \lambda_{j} C_{i j}-2 \sum_{i=0}^{n} \lambda_{i} C_{i 0}+C_{00}
$$


Semivariogram Semivariogram is used for modeling the similarity points in a field as a function of distance and it can be given as Equation 2.19 and drives to Equation 2.20

$$
r_{i j}=\frac{1}{2 N(h)} \sum_{N(h)}\left(z_{i}-z_{j}\right)^{2}
$$

Where $\mathrm{N}$ is the number of data points in the distance of $h$.

$$
\begin{aligned}
r_{i j}=\frac{1}{2} E\left[\left(Z_{i}-z_{j}\right)^{2}\right] & =\frac{1}{2} E\left[\left(R_{i}-R_{j}\right)^{2}\right]=\frac{1}{2} E\left[R_{i}^{2}-2 R_{i} R_{j}+R_{j}^{2}\right] \\
& =\frac{1}{2} E\left[R_{i}^{2}\right]+\frac{1}{2} E\left[R_{j}^{2}\right]-E\left[R_{i} R_{j}\right]=\sigma^{2}-C_{i j}
\end{aligned}
$$

Again, the object function $J$ can be expressed as Equation 2.21 with constrain as Equation 2.14

$$
\begin{array}{r}
J=\sum_{i=0}^{n} \sum_{j}^{n} \lambda_{i} \lambda_{j}\left(\sigma^{2}-r_{i j}\right)-2 \sum_{i=0}^{n} \lambda_{i}\left(\sigma^{2}-r_{i j}\right)+\left(\sigma^{2}-r_{o o}\right) \\
=\sigma^{2}-\sum_{i=0}^{n} \sum_{j}^{n} \lambda_{i} \lambda_{j}\left(r_{i j}\right)-2 \sigma^{2}+2 \sum_{i=0}^{n} \lambda_{i}\left(r_{i o}\right)+\sigma^{2}-r_{o o} \\
=2 \sum_{i=0}^{n} \lambda_{i}\left(r_{i o}\right)-\sum_{i=0}^{n} \sum_{j}^{n} \lambda_{i} \lambda_{j}\left(r_{i j}\right)-r_{o o}
\end{array}
$$

Where $r_{i j}$ is the function $r=r(h)$ by distance $h_{i j}$, which separates the two points $z_{i}$ and $z_{i=j}$. The semivariogram could be fitted by various models such as the spherical, exponential, and the Gaussian. The best fitting model with optimal value of parameters is determined by the measurement, for instance, Mean Square Error as illustrated in Equation 2.33 .

In order to find the optimal $\lambda$, the method of Lagrange multipliers is employed for finding the local minima of a function subject to equality constraints. Consequently, a new objective function is generated as following equation:

$$
J+\Phi\left(\sum_{i=0}^{n} \lambda_{i}-1\right)
$$


The partial derivative of objective function updateObjective subject to constraint with each variable $\lambda_{i}$ and $\Phi$ is computed as Equation 2.23 .

$$
\begin{array}{r}
\frac{\partial\left(J+\Phi\left(\sum_{i=0}^{n} \lambda_{i}-1\right)\right)}{\partial \lambda_{i}}=0 ; i=1,2, \ldots, n \\
\frac{\partial\left(J+\Phi\left(\sum_{i=0}^{n} \lambda_{i}-1\right)\right)}{\partial \Phi}=0 \\
\left\{\begin{array}{l}
r_{11} \lambda_{1}+r_{12} \lambda_{2}+\ldots+r_{1 n} \lambda_{n}=r_{10} \\
r_{21} \lambda_{1}+r_{22} \lambda_{2}+\ldots+r_{2 n} \lambda_{n}=r_{20} \\
\ldots \\
r_{n 1} \lambda_{1}+r_{n 2} \lambda_{2}+\ldots+r_{2 n} \lambda_{n}=r_{n 0} \\
\lambda_{1}+\lambda_{2}+\ldots+\lambda_{n}=1
\end{array}\right.
\end{array}
$$

Based on the Equation above, the vector $\lambda$ for minimizing the objective function(Equation 2.22 can be found. Accordingly, the value for the point $\left(x_{o}, y_{o}\right)$ is interpolated.

\section{Time series (TS)}

The main characteristic of the time series (TS) modeling is that it only tries to model the relationship between each observation with the previous observations. Thus, the predicted values are modeled as a function of the previous values or as a function of the position in the time [123]. One basic and relevant requirement of this model is that the data series must be stationary. This is generally achieved with: (i) data transformation and/or (ii) data detrending and/or (iii) data differencing (firstand second-order) before applying the Fourier decomposition. As the input variables to time-series models are only lagged values of response, the performance of this model concerning prediction is weak[108].

Autoregressive integrated moving average $(\operatorname{ARIMA}(\mathrm{p}, \mathrm{d}, \mathrm{q}))$ is one type of time series techniques that have been widely applied on forecasting. A correlogram is needed for determining whether a particular series is stationary or non-stationary. Usually, a stationary time series will give an autocorrelation function (ACF) that decay rapidly from its initial value of unity at zero lag. In the case of non-stationary time series, the ACF dies out gradually over time. If the values are observed as non- 
stationary, differencing is need for making this non-stationary time series become stationary. The value of difference $(\mathrm{d})$ is determined by the number of times the differencing is performed on the time series.

In forecasting form, the best model can be expressed as Equation 2.25.

$$
Y_{t}=\phi Y_{t+1}+\theta+\varepsilon_{t}
$$

\section{Single ML techniques}

\section{Artificial Neural network(ANN)}

ANNs are computational models inspired by animals' central nervous systems (in particular the brain) that are capable of machine learning and pattern recognition. They are usually presented as systems of interconnected "neurons" that can compute values from inputs by feeding information through the network. Natural neurons receive signals through synapses located on the dendrites or membrane of the neuron. When the signals received are strong enough (surpass a certain threshold), the neuron is activated and emits a signal through the axon. This signal might be sent to another synapse, and might activate other neurons.

The complexity of real neurons is highly abstracted when modeling artificial neu-

rons. These basically consist of inputs (like synapses), which are multiplied by weights (strength of the respective signals), and then computed by a mathematical function which determines the activation of the neuron. Another function (which may be the identity) computes the output of the artificial neuron (sometimes in dependance of a certain threshold). ANNs combine artificial neurons in order to process information[124].

Since the first neural model by McCulloch and Pitts[125], there have been developed hundreds of different models considered as ANNs. The differences in them might be the functions, the accepted values, the topology, the learning algorithms, etc. Also, there are many hybrid models, where each neuron has more properties than the ones we are reviewing here.

Based on the criteria - connectionism, ANNs can be classified into three types: 
Feed-forward Network, Recurrent Network and Reinforcement Network. Feed-forward Network is the most popular. Recurrent Network is getting more attention in recently years. However, rare researchers applied Reinforcement Network in pollutant prediction modeling.

From learning algorithm perspective, ANN are categories of supervised learning network, unsupervised learning network, hybrid learning network, associate learning network and optimization application network. The weights between the layers are adjusted at each iteration by the feedback of the squared error cost function, which is computed by predicted and real outputs. The weights are optimized by minimizing error after many iterations, which makes the algorithm called supervised learning [126]. Back propagation algorithm is one of the most common models used for supervised learning network.

In this research, feed-forward back propagation network(FBPN) is selected as ANN structure for the prediction. Accordingly, the description of ANN method afterward will focus on the mechanism of FBPN. The structure of FBPN consists of three layers: input layer, hidden layer, and output layer as shown in Figure 2-13. The neurons of the input layers consist of the input variables. The output layer is connected with the output variable. The numbers of the hidden layer could vary. According to the study [126, nevertheless, one hidden layer with many neurons as ANN architecture can yield promising results. Each node in the hidden layer is a neuron (or processing element) with an either linear or non-linear activation. However, an ANN with linear activation functions in the hidden layer is equivalent to a linear model with limited abilities. Therefore, it is of necessity to use non-linear activation function in order to enable ANN to capture the complexity and non-linear relationships within a system.

The size of nodes in the hidden layer and weigh decay are the two critical parameters that influence the modeling performance. The size of nodes in the hidden layer is extended from 10 to 50 in order to fully train the models, generate the best ANN structure, and then yield an improved model performance. However, large size of nodes in hidden layer may lead to over-fitting issue. In such case, weigh decay is 


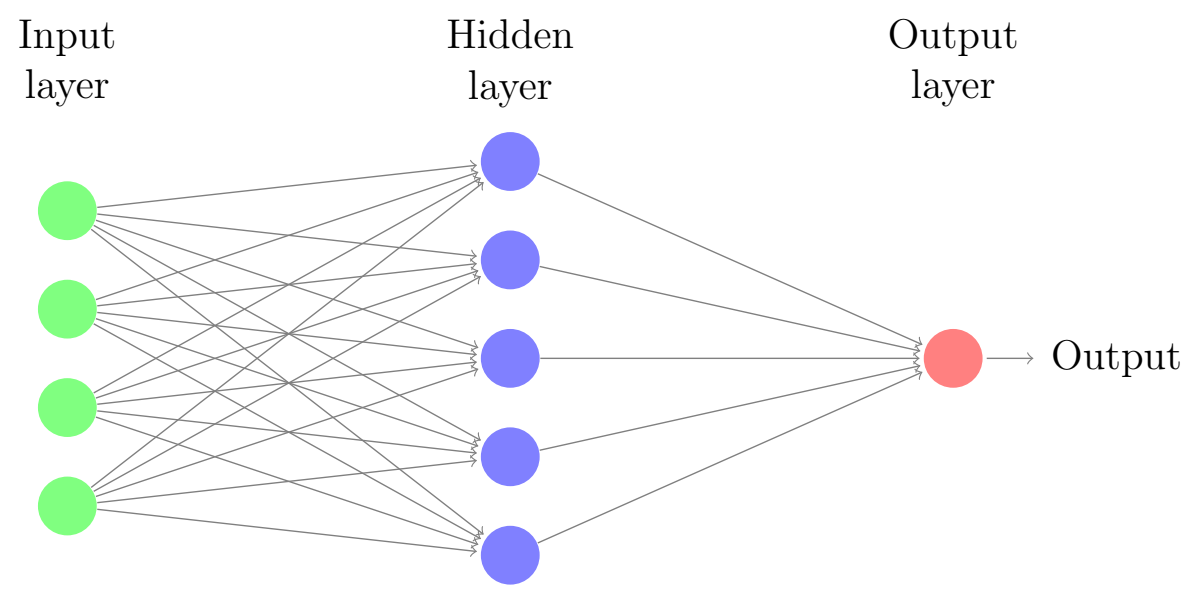

Figure 2-13: FFBP structure

tuned for penalizing the large weight and regularizing the cost function.

\section{Support regression vector(SVM)}

Since 1990, SVM was invented and gradually replace ANN. SVM was firstly used for classification, but later generalized to the regression problems. SVM can be classified into two types: no kernel(linear kernel) and with kernel Take SVM with linear kernel as example, SVM aims to find optimal separating hyperplane with maximum margin as shown in Figure 2-14. Regarding to a dataset that it is not linearly separable, the non-linear kernel with some functions, such as radial basis function, polynomial and sigmoidal, can project the input to a higher feature space to make it linear separable.

More formally, a support vector machine constructs a hyperplane or set of hyperplanes in a high- or infinite-dimensional space, which can be used for classification, regression, or other tasks. Intuitively, a good separation is achieved by the hyperplane that has the largest distance to the nearest training data point of any class (so-called functional margin), since in general the larger the margin the lower the generalization error of the classifier. Whereas the original problem may be stated in a finite dimensional space, it often happens that the sets to discriminate are not linearly separable in that space. For this reason, it was proposed that the original finite-dimensional space be mapped into a much higher-dimensional space, presumably making the separation easier in that space. To keep the computational load reasonable, the mappings 
used by SVM schemes are designed to ensure that dot products may be computed easily in terms of the variables in the original space, by defining them in terms of a kernel function $K(x, y)$ selected to suit the problem. The hyperplanes in the higherdimensional space are defined as the set of points whose dot product with a vector in that space is constant. The vectors defining the hyperplanes can be chosen to be linear combinations with parameters $\alpha_{i}$ of images of feature vectors that occur in the data base. With this choice of a hyperplane, the points $\mathrm{x}$ in the feature space that are mapped into the hyperplane are defined by the relation: $\sum_{i} \alpha_{i} K\left(x_{i}, x\right)=$ constant. Note that if $K(x, y)$ becomes small as y grows further away from $\mathrm{x}$, each term in the sum measures the degree of closeness of the test point $\mathrm{x}$ to the corresponding data base point $x_{i}$. In this way, the sum of kernels above can be used to measure the relative nearness of each test point to the data points originating in one or the other of the sets to be discriminated. Note the fact that the set of points $\mathrm{x}$ mapped into any hyperplane can be quite convoluted as a result, allowing much more complex discrimination between sets which are not convex at all in the original space

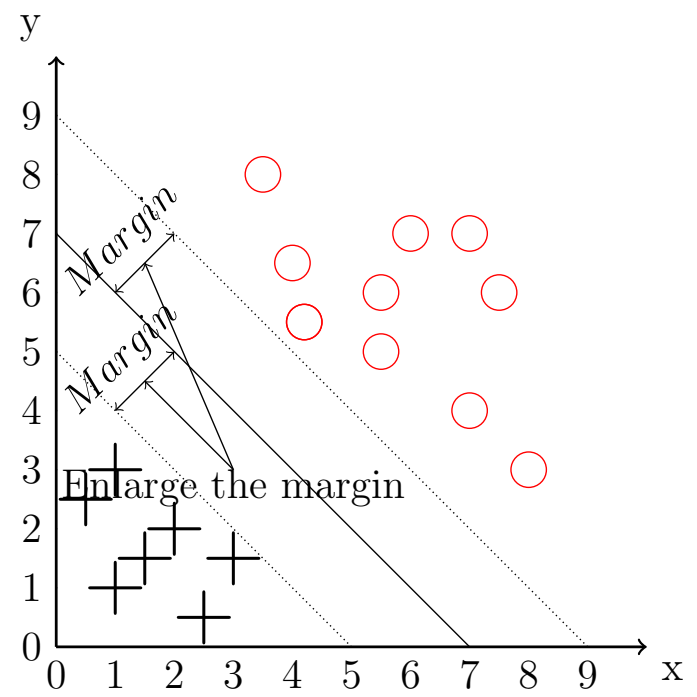

Figure 2-14: Optimal hyper-plane in SVM(linearly seperable case)

\section{The Classification and Regression Tree}

The Classification and Regression Tree (CART) method was first introduced by Breiman in 1984 [127, 37]. It is a form of binary recursive partitioning. Initially, the data (represented by the parent node) are split into subsets (child nodes) based on 
different evaluation functions (Gini diversity index, the entropy, or the error index) [128]. Then, the method subjects each subset (child node) to the same partitioning process until only a few samples remain in the terminal subset (nodes).

Normally, each node corresponds to a variable. In such case, the importance score for a variable, when it is used as a splitter, can be determined by summing the improvement scores(e.g. Gini) of all nodes. In this regard, tree-based algorithms are always employed for variable importance rank and variable selection. The CART-base learners including CART, Bagging, GBM, and RF, therefore, are used for variable importance analysis.

\section{Comparison of single models}

As compared to the ANN, the SVM has a better generation capability, global optimization, and dimensional independence [129, 130]. In addition, empirical comparisons have shown that the SVM performs faster in the training set than ANN. However, ANN are more robust to noise than the SVM [131]. CART is a non-parametric data-driven technique, i.e, the number of parameters is not specified before modeling. As compared with ANN and SVM, CART is a faster and more versatile predictive algorithm.

\section{Random Forest $(\mathrm{RF})$}

Random Forest $(\mathrm{RF})$ algorithm is based on the classification and regression tree(CART) which was first introduced by the Leo Breiman [132]. It is an ensemble algorithm that applies bagging methods on CART. Different from CART, RF selects only a few features randomly in each training time. In this way, RF could get a lower error level and running time rather than using all the input variables[132]. Then, it weights each feature, sorts them and selects the most important features. The performance function is the measurement of model performance, this work used RMSE2.34. Because it weights importance of each feature, $\mathrm{RF}$ is always a popular algorithm for feature selection. For the RF models training, only parameter in this study is the numbers of the trees. It range from 20 to 40 . The best value parameter is selected based on the RMSE errors. 


\section{Ensemble ML techniques}

Ensemble algorithms are aimed to construct a set of classifiers or regressors to build a more robust and higher-performance classifier or regressor [133]. Bagging and boosting are the two main ensemble methods, which can be merged with basic learners. The basic learners should not be "stable"; such a learner was called a "weak learner" in previous papers [134, 135]. The weak learner is characterized by the fact that small changes in the training data can cause a large change in the prediction in the test set. As compared to ANN and SVM, CART is much "weaker." Therefore, it has been widely used in existing studies and has shown promising results in various applications [134, 135, 64, 136]. Although a study adopting ANN and SVM as basic learners was conducted, a comparison showed that the CART ensembles with boosting outperform SVM and ANN on average [137]. Therefore, in our research, CART was finally selected as the basic learner for both the bagging and boosting algorithms.

\section{Bagging: Bagging CART and RF}

Bagging is a traditional technique used in ensemble methods. It randomly resamples the data $k$ times with replacement. Then, it trains learners for each $k$ subset sample by using CART. Finally, all the learners are aggregated by a majority vote for classification [64].

Random Forest (RF) was first introduced by Breiman. Like the bagging CART, it is also an ensemble algorithm, combining bagging and CART. However, in contrast to the bagging CART mentioned above, RF selects only a few features randomly during each training, thereby achieving a lower error level and requiring less running time than when all the features are used [132, 37].

\section{Boosting: Adaboost and GBM}

Boosting is one of the most powerful machine learning algorithms developed in the past few years. The original idea of boosting came from the probably approximately correct (PAC) learning framework [138]; it is further explained in [139] and its design was first presented in [140].

The idea of the boosting algorithm is that it generates a series of basic learners by 
re-weighting the samples in the training sets. Specifically, during the training process, the misclassified examples are higher weighted while the correctly classified samples are lower weighted. The new samples are adjusted for the next training round. The adjusted samples, by which various individual learners are trained in each round, can then diversify the classifiers. Finally, the new learner, which is constructed by combining these individual learners, can be obtained by weighted or simple voting schemes [141, 142, 143].

Boosting CART is a method that combines boosting with CART. The boosting algorithm has various forms with different loss functions. AdaBoost, with an exponential loss function and the generalized boosted model (GBM), with a gradient descent algorithm, are the two most widely used forms in boosting. More details about this algorithm can be found in [144].

\section{Stacking}

In contrast to the boosting and bagging methods, which always involve the same learner, stacking is another ensemble method that involves different types of learners [143. Stacking ensemble is a simple ensemble algorithm that trains a "predictor of predictors." This algorithm sees individual predictors (models) as features and then finds a combination of these predictors by using different algorithms.

In this study, the L-Ensemble, short for Linear Ensemble, is one of the stacking ensemble methods. A study of Wolpert conducted in 1992 showed an idea that instead of selecting one from a set of predictors, a predictor could be obtained by combining them and notably decrease the prediction error. This idea was supported in [145]. Stacking methods by linearly combining all the individual predictors (in our case the predictors are SVM, ANN, RF, CART, GMB, AdaBoost, and Bagging), could enhance the prediction accuracy. The basic idea is to use cross-validation data and least squares under non-negativity constraints to determine the coefficients in the combination.

\section{Comparison of ensemble models}

The main strengths of the three ensemble algorithms is that they are fast and simple methods that are easy to program. No prior knowledge about the weak learner 
is required. Bagging can significantly reduce variance and then improve the prediction accuracy; however, it is not an effective method for reducing bias. Boosting, on the other hand, can reduce both variance and bias. Meanwhile, it is capable of identifying the extreme values, or the mislabeled samples in the training data, or the samples that are not easily classified, since boosting always focuses on these samples by giving them a higher weight for the next training round.

\section{Deep learning}

Deep learning has obtained many successes in the application of image classification, natural language processing and speech recognition. It can extract features

Deep learning is a new technique that have been obtained attraction by many scholars in various domains. The condition of applying deep learning to work well is that requirement of "relatively" large data sets. Moreover, deep learning need more computation than traditional artificial intelligence algorithms such as random forest, SVM. However, one of the most famous advantages of deep learning is that feature engineering part have been involved inside this algorithm, that is, the features extraction can be realized by building multiple hidden layers.

During the training, a few of parameters are required to tuned, based on which different network topologies can be built and the forecasting accuracy should be different. In our study, the main three parameters were chosen to be tuned, which are network size, dropout, and epochs.

\subsubsection{Parameters tuning}

ANN and SVM are the two of most popular and powerful algorithms for both classification and regression. However, one of their disadvantages is that they are sensitive to their own architectures that are determined by various combinations of parameters. In other words, in order to obtain a performing forecasting results, some key parameters are necessary to be tuned get the optimal combination of parameters.

Take ANN as example, the architecture consists of three layers as described before. 
The input layer has been determined by the input variables while the output layer by the output variable. Accordingly, the parameters related to hidden layers are of the most importance and key ones that influence the modelling performance. Therefore, some relevant aspects for ANN architecture are generated as follows:

- How many hidden layers should be included?

- Which type of the hidden layer should be used for the units in the hidden layers.

- How many nodes should be included for each hidden layer.

- How to avoid the over-fitting problem

There is no a particular rule to decide how many hidden layers that a ANN should have, however, according the previous, one hidden layer with many neurons still can yield promising results as described in the study [126]. Therefore, this study chooses three layers as the ANN structure.

Instead of using linear activation function, the non-linear active functions are more attractive and effective for solving the non-linear problems. The two main activation function sigmoid and Radial basis function.

Regarding the numbers of units in the hidden layers, there is no strict role to making decision. But the most commonly comment is that the optimal size of the hidden layer is usually between the size of the input and output. Still there some other rules said that the number of hidden neurons not only depends on numbers of neurons in input and output layer, but also consider the number of samples in the training dataset. For example, as description in the following equation, alpha is usually scaling from 2 to 10 . The training samples $N_{s}$ in the Hong Kong case are 2149 , numbers of inputs $N_{i}$ is 22 and output $N_{o}$ is 1 respectively. Therefore, maximum $N_{h}$ should be around 50. Then this is the reason that the parameter size is tuned from 1 to 50 .

$$
N_{h}=N_{s} /\left(\left(\operatorname{alpha} *\left(N_{i}+N_{o}\right)\right)\right)
$$

To avoid the over-fitting problem(high variance), on the one hand, we employed the three-fold cross validation for training models; on the other hand we used regular- 
ization parameter to penalize the corresponding weights. In this study, the parameter decay corresponds to the penalization. However, if the decay is too large, there could be a large bias. Therefore, we change the decay parameters $0.0001,0.001,0.01$, and 1 to test the best decay parameters for the ANN

The parameters, such as learning rate which is control the learning speed for gradient descent to minimize the cost function, should be interesting. However, learning speed, as long as not set it too large, the optimal weights could be found just in matter of computing time. This study tried this parameter as well in our experiment and there is no significant differences using different learning rates. Therefore, we did not include it in our paper. We believe the numbers of neurons in the hidden layer as well as the regularization are the most importance parameters, which should be highlighted.

For SVM algorithm, the process of considering parameters is the same as ANN. In addition, similar to the parameter weight decay for ANN and cost for SVM, complexity for CART is the one that control the trade-off between model generalization and low error of modelling for training data.

\subsubsection{Cross validation}

In order to avoid overfitting and increase the model generalization capability, cross validation techniques were applied in this study. There are three types of crossvalidation method: random subsampling, K-fold cross validation, and leave-one-out validation. The K-fold cross validation method is the most widely used, since it is simple and easy, and uses all the data for training and validation [109]. In this monograph, the entire training and validation data sets were randomly partitioned into three equal sized subsamples, two of which were used for training and parametertuning purposes, and one for validation. This was repeated three times (three-fold) until all the three subsamples were used as validation data. Finally, the errors rates of three experiments were averaged. 


\subsubsection{Performance measurement}

According to the two modes of prediction involved, that is, classification mode and regression mode, the ways to evaluate the models' performance are different. The various measurements for classification mode considering unbalance problem, and for regression mode are introduced as following subsections.

\section{Classification measurement}

Many studies on comparative classification algorithms used measurements such as accuracy and precision/recall for evaluating model performance. However, these measurements are more suitable for cases with balanced class distribution. Concerning the imbalance problem, the minority cases have little influence on these measurements as compared to the majority cases [146, 147]. Therefore, some measurements were carried out when the minority class was of major interest. $G_{\text {mean }}$ (Equation 2.30, weighted accuracy(WA) (Equation 2.31), and $F_{\text {measure }}$ (Equation 2.32 which can trade-off the performances of both minority and majority classes were selected as performance measures. Based on the confusion observed in the matrix in Table 2.14. the equations for each measurement are defined as follows:

$$
\begin{gathered}
\text { TrueNegativeRate }\left(A C C^{-}\right)=\frac{T N}{T N+F P} \\
\text { Truepositiverate }\left(A C C^{+}\right)=\frac{T P}{T P+F N} \\
\text { Precision }=\frac{T P}{T P+F P} \\
\text { Recall }=\frac{T P}{T P+F N} \\
G_{\text {mean }}=\left(A C C^{+} * A C C^{-}\right)^{\frac{1}{2}}
\end{gathered}
$$


Table 2.14: Confusion matrix

\begin{tabular}{lll}
\hline & predict positive & predict negative \\
\hline Actual positive & $\mathrm{TP}($ True positive) & $\mathrm{FN}$ (False negative) \\
Actual negative & $\mathrm{FP}($ False Positive) & $\mathrm{TN}$ (True negative) \\
\hline
\end{tabular}

$$
\begin{gathered}
\text { WeightedAccuracy }=\beta * A C C^{+}+(1-\beta) * A C C^{-} \\
F_{\text {measure }}=\frac{2 * \text { Precision } * \text { Recall }}{\text { Precision }+ \text { Recall }}
\end{gathered}
$$

\section{Regression measurement}

Quantitative error measure are often used for judging the predictive model performance. mean Square Error(MSE), Root mean Square Error(RMSE), and Relative Estimate for Linear Error(RELE) are three of the key measurement methods. The formulas are listed from Equation 2.33 to Equation 2.35. RMSE is the square root of MAE, which is consistent with the units of original data. MAE is similar to RMSE. An explication of these error measurements can be found in the study [148]. Consider the pros and cons of each methods and reflection of modeling quality from different aspects, this study prefers RMSE, MAE, and RELE as the error measurement for evaluating predictive model performance.

$$
\begin{gathered}
M A E=\frac{1}{n} \sum_{i=1}^{n}\left|y_{i}-\hat{y}_{i}\right| \\
R M S E=\sqrt{\frac{1}{n} \sum_{i=1}^{n}\left(y_{i}-\hat{y}_{i}\right)^{2}} \\
R E L E=\frac{1}{n} \sum_{i=1}^{n} \frac{\left|y_{i}-\hat{y}_{i}\right|}{\hat{y}_{i}}
\end{gathered}
$$


Where $y_{i}$ are the prediction values, and $\hat{y}_{i}$ are the observed values.

\subsubsection{Statistical comparison}

A statistical test is conducted to compare the average values in terms of the three measurements for the different models. In the existing studies, two statistical test have been used, namely Student's t-test[149] and the Wilcoxon Rank-Sum test (WRS) [150]. The former is a parametric statistical test, which is uniformly the most powerful unbiased test under normal curve theory. The latter one is a nonparametric test without assumptions on the probability distribution. A study from [151] evaluates these two statistical tests under varying conditions, such as the data distribution, size, and samples, by using real-world data from the medical domain. The recommendation is given to WRS when the characteristics are small and nonsymmetric. Therefore, considering the characteristics of the models, WRS is employed for the statistical test in this study.

\subsubsection{Cluster algorithm}

The traditional cluster algorithms can be categorized into partitioning methods, hierarchical methods, density-based methods, grid-based methods, model-based methods. This study selects hierarchical methods(Agglomerative) and model-based methods(Self Organized Maps and Gaussian Mixture Models) for further analysis.

\section{Agglomerative hierarchical clustering}

Hierarchical cluster algorithm is a method of cluster analysis which seems to build a hierarchy of clusters. Based on the strategy, it falls into two types: Agglomerative and divisive. The former one is a "bottom-up" approach starting its own cluster for each variable, and then the pairs of clusters are merged as one moves up the hierarchy; The latter one refers to "top-down" approach,that is, all the variables belong to one cluster, afterward split recursively as one moves down the hierarchy. This study will use agglomerative hierarchical clustering. 
Metric and linkage criteria are the two determines for the clustering results. Metric is the measurement of the distance between pairs of observation. Metric determines directly the shape of clustering. There are several metrics widely used in the hierarchical clustering algorithms such as Euclidean distance, Square Euclidean distance, Manhattan distance etc. Linkage criteria are the clustering rules that are used for determining the distance between two sets of observations as a function of the pairwise distances between observations, mainly including maximum or complete -linkage clustering, minimum or single-linkage clustering, average linkage clustering, and centroid linkage clustering.

This work will use Euclidean distance and centroid linkage clustering presented in Equation 2.36 and 2.37 for the hierarchical clustering algorithm.

$$
\begin{gathered}
\|a-b\|=\sqrt{\sum_{i=1}^{n}\left(a_{i}-b_{i}\right)} \\
\left\|c_{s}-c_{t}\right\|
\end{gathered}
$$

where $c_{s}$ and $c_{t}$ is the centroid of cluster $s$ and $t$, respectively.

\section{Self-organizing $\operatorname{map}(\mathrm{SOM})$}

Self-organizing map is designed for addressing unsupervised learning problems such as feature extraction, clustering, and visualization. It is inspired by postulated feature maps of neurons in the brain comprised of feature-sensitive cells that provide ordered projection between neural layers, such as those that may exist in the retina and cochlea.

The objective of SOM is to optimally place a topology of codebook or prototype vectors in the domain of the observed input data samples. It is comprised of a collection of codebook vectors connected in a topological arrangement, typically a one dimensional line or two dimensional grid. The result projects in lower dimensions, or approximates the problem domain which can be visualized as well, or from which clusters could be extracted. 
The winner neuron determines the spatial location of neighborhood of excited neurons, and then enables the excited neurons to increase their individual values of the discriminant function in relation to the input pattern. The operations of SOM, then, can be summarized as follows:

- Select random input.

- Compute the winner neuron.

- Update neurons.

- Repeat for all input data.

- Classify input data.

To illustrate the algorithm of SOM in detail, Table 2.15 provides the pseudocode for the SOM method.

\section{Gaussian Mixture MDodels}

Gaussian mixture models (GMM) are often utilized for data clustering. GMM is consisted of K components(K Gaussian Distribution $N\left(x \mid \mu_{k}, \sum_{k}\right)$. Probability density function can be illustrates as Equation 2.38 .

$$
p(x)=\sum_{k=1}^{k} p(k) p(x \mid k)=\sum_{k=1}^{k} \pi_{k} N\left(x \mid \mu_{k}, \sum_{k}\right)
$$

Then, the parameters of GMM includes $\pi_{k}, \mu_{k}$, and $\sum_{k}$. The idea is to find a combination of the parameters to achieve the highest probability with the data points $\operatorname{Max}\left(\Pi_{i=1}^{N} p\left(x_{i}\right)\right)$, where $N$ is the number of given data and points and $p_{x}$ is the density probability distribution for all the data points. $\Pi_{i=1}^{N} p\left(x_{i}\right)$ is also named Likelihood function. In order to maximum the the Likelihood function, a ExpectationMaximization is employed.

$$
N(x)=|2 \pi \Sigma|^{-\frac{1}{2}} \exp \left\{-\frac{1}{2}(x-\mu)^{t} \Sigma^{-1}(x-\mu)\right\}
$$


Table 2.15: The pseudocode for Self-Organizing Map Algorithm

Algorithm : Self-Organizing Map

\section{Input: parameters}

Learning rate $M$; Numbers of Interactions $\delta$; Width of Grid Grid width

Height of Grid Grid $_{\text {height }}$; Neighborhood size Nh

\section{Algorithms:}

//Initialization: The $m$ dimensions input $\vec{x}=\left[x_{1}, x_{2} \ldots \ldots x_{n}\right]$

$\vec{x}_{n o r m}=$ Normalize $\left(\left[x_{1}, x_{2} \ldots \ldots x_{n}\right]\right)$

//Generate random codebook vectors to small floating point values, or sampled from domain

$$
\overrightarrow{w_{l}}=\left[w_{l 1}, w_{l 2} \ldots . w_{j m}\right], l=1,2, \ldots, l \text { connect } l \text { numbers of output }
$$

//Measure the Euclidean distance between input vector with $n$ number of attributes and a given codebook vector $c$

$$
\operatorname{dist}(x, c)=\sum_{i=1}^{n}\left(x_{i}-c_{i}\right)
$$

// Calculate the Best Matching Unit(BMU)(The codebook vector from the pool that has the minimum distance to an input vector)

For $(\mathbf{i}=0$ to $\delta)$

$$
\begin{aligned}
& M_{i+1} i^{-} \text {updateLearningRate }\left(M_{i}\right) \\
& \text { //Calculate Neighborhood Size }
\end{aligned}
$$$$
N h_{i+1}=\text { updateNeighborhoodSize }\left(N h_{i}\right)
$$

// All neighborhood nodes are updated using the same learning rate for the iteration.

\section{For( Vector $_{i} \in$ Neighborhood}

For $\left.\operatorname{Vector}^{j}\right) j=1 \ldots m$

$$
\text { Vector }^{j}=\text { Vector }^{j}+M_{i+1} *\left(\operatorname{Pattern}_{i}^{j}-\text { Vector }_{i}^{j}\right)
$$$$
c_{t+1}=\delta_{t} *\left(c_{i}(t)-x_{i}\right)
$$

// Minimizing the vector of $\vec{x}-\vec{w}_{j}$

endFor

endFor 
Equation 2.39 shows the multivariate normal distribution. $\Sigma^{-1}$ is covariance matrix that determines the shape of the clusters. There is a trade-off between the shape of cluster and numbers of clusters, that is, if the shape of the clusters is simple, more clusters should be used for better clustering, and vice verse.

Deciding the number of cluster is crucial for the further scenario work or being tested by other external data 90 . In this study, the optimal number of clusters is in light of Bayesian Information Criterions (BIC) for expectation maximization method. $B I C=-2 \log (L)+m \log (n)$, where $L$ is the likelihood function and $\mathrm{m}$ is the number of free parameters to be estimated. A model with low $B I C$ superior to the ones with high $B I C$. One the models listed in Table 2.16 based on the $B I C$ measurement

Table 2.16: Candidate models for selection

\begin{tabular}{|c|c|c|c|}
\hline $\mathrm{E}:$ & Equal variance(unvariate) & EVI & $\begin{array}{l}\text { Diagonal, equal volume and } \\
\text { shape }\end{array}$ \\
\hline $\mathrm{V}$ & $\begin{array}{l}\text { Variable } \\
\text { ance(univariate) }\end{array}$ & VVI & $\begin{array}{l}\text { Diagonal, varying volume } \\
\text { and shape }\end{array}$ \\
\hline EII & Spherical, equal volume & EEE & $\begin{array}{l}\text { Ellipsoidal, equal volume, } \\
\text { shape and orientation }\end{array}$ \\
\hline VII & Spherical, unequal volume & EEV & $\begin{array}{l}\text { Ellipsoidal, equal volume } \\
\text { and equal shape }\end{array}$ \\
\hline EEI & $\begin{array}{l}\text { Diagonal, equal volume and } \\
\text { shape }\end{array}$ & VEV & Ellipsoidal, equal shape \\
\hline VEI & $\begin{array}{l}\text { Diagonal, equal volume, } \\
\text { varying shape }\end{array}$ & VVV & $\begin{array}{l}\text { Ellipsoidal, varying volume, } \\
\text { shape, and orientation }\end{array}$ \\
\hline
\end{tabular}




\subsubsection{Calculation for immaterialisation, dematerialization, and decarbonisation}

Due to lack of $\mathrm{CO}_{2}$ emission statistic in China, $\mathrm{CO}_{2}$ will be calculated by the types of energy and their corresponding $\mathrm{CO}_{2}$ emission conversion factors provided by the Intergovernmental Panel on Climate Change(IPCC) database of year 2006[152], which was done by most of the existing studies [88, 89]. The Calculation of the $\mathrm{CO}_{2}$ conversion for each province $j$ is presented as Equation 2.40.

$$
\left(\mathrm{CO}_{2}\right)_{j}=\frac{44}{11} \sum_{i=1}^{n} K_{i} E_{i}
$$

Where $K_{i}$ is the conversion factor $K$ for energy type $i$. $E$ is the energy consumption for energy type $i$; There are 18 types of energy included and listed in the Table 2.17.

Table 2.17: $\mathrm{CO}_{2}$ Conversion factors for each type of energy

\begin{tabular}{|c|c|c|c|c|c|c|c|c|c|}
\hline \multirow[t]{3}{*}{ Items } & Raw & Cleaned Coal & Other & Brique & Coke & Coke & Other & Crude & Gasoline \\
\hline & \multirow[t]{2}{*}{ Coal } & & Washed & & & Oven & Gas & Oil & \\
\hline & & & Coal & & & Gas & & & \\
\hline \multicolumn{2}{|c|}{ Coefficient 2.69} & 3.09 & 1.98 & 1.55 & 3.14 & 0.93 & 0.88 & 2.76 & 2.26 \\
\hline \multirow[t]{4}{*}{ Items } & \multirow[t]{4}{*}{ Kerosene } & \multirow[t]{4}{*}{ Diesel Oil } & \multirow[t]{4}{*}{ Fuel Oil } & \multirow[t]{4}{*}{ LPG } & Refinery & Natural & Other & Other & Other \\
\hline & & & & & \multirow[t]{3}{*}{ Gas } & \multirow[t]{3}{*}{ Gas } & \multicolumn{2}{|c|}{ Petroleum Coking } & Energy \\
\hline & & & & & & & Prod- & Prod- & \\
\hline & & & & & & & ucts & ucts & \\
\hline Coefficient & 2.56 & 2.73 & 2.39 & 1.75 & 2.98 & 2.09 & 2.76 & 3.14 & 2.74 \\
\hline
\end{tabular}

After obtaining $\mathrm{CO}_{2}$ emission, energy consumption, the energy consumption for each province can be aggregated by Equation 2.41.

$$
E_{p}=\sum_{i=1}^{n} E_{i}
$$

Using the GDP, energy consumption, and $\mathrm{CO}_{2}$ consumption data, the immateri- 
alisation, dematerialization, and decarbonisation for each province are identified as Equation 2.42 - 2.44 respectively.

$$
\begin{gathered}
\text { immaterialisation }=\frac{E_{y 1} / G D P_{y 1}}{E_{y 2} / G D P_{y 2}} \\
\text { dematerialization }=\frac{\left.\left(C O_{2}\right)_{y 1} / E_{y 1}\right)_{y 2}}{\left(C O_{2} / E_{y 2}\right.} \\
\text { decarbonisation }=\frac{\left(C O_{2}\right)_{y 1} / G D P_{y 1}}{\left(C O_{2}\right)_{y 2} / G D P_{y 2}}
\end{gathered}
$$

\subsection{Tools and platform}

Due to the multiple tasks involves in, various tools are used for corresponding task depending on their features. For this study, three software and two platforms are mainly utilized including MySQL for database management, $\mathrm{R}$ for data processing, statistical computing and machine learning modelling, Stata for statistics analysis, and Python for scraping messages from web and social media. In the follows, the details of these tools along with the corresponding applied study are described shortly.

\subsubsection{MySQL database}

MySQL is free SQL client/server relational database management system. It includes an SQL server, client programs for accessing the server, administrative tools, and a programming interface for writing your own program. MySQL is portable and runs on commercial operation system(Mac OS, Unix and Windows) and on hardware all the way up to the enterprise servers. It can also handle large databases with billions of rows .

By comparing with other database management systems such as SQLite and PostgreSQL, MySQL is faster, friendly use, and can provide interfaces for many languages like C, Java, PHP, Python, R. The lowering of the economic barriers to computing is a factor be chosen for many organizations. 
For the reasons aforementioned, MySQL is selected as database management system. The system was installed on 24 cores Linux system with processor model AMD Operaon 6234. All the data selected from corresponding data sources mentioned in Section 2.1 are stored in MySQL(Figure 2-15). R can connect MySQL with the RMySQL package, in which way let us enter queries and view the results directly in $\mathrm{R}$ environment.

\begin{tabular}{|c|c|c|c|c|c|c|c|c|c|c|c|c|c|c|}
\hline phoMuAdmin & 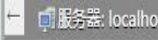 & 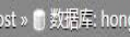 & long 》 & $=$ & eo & & & & & & & & & th \\
\hline 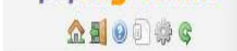 & 圆测览 $\mid k$ 结构 & \&SQL Q & 搜索 羿 & 插入 & $\Rightarrow$ 封出 & & a: & 限操作 & 乎触 & & & & & \\
\hline 近䎳汸问 表收藏夹 & $\leftarrow T \rightarrow$ & ID & FECHA & HORA & ESTACION & WD & Ws & CLD GUST & TEMP & WET & DEW & RH & MSLP & RF TIMESTAMP \\
\hline & (1) D编辑 音: 复制 & 1) (2) 那除 234863 & 1989-08-01 & 1 & 1 HKS & 290 & 0.4 & NULL 1.9 & 25.7 & 23.8 & 23.0 & 85 & N.A. & N.A. 2014-09-03 15:38:16 \\
\hline $\begin{array}{l}+-1 \text { asasec } \\
+-3 i b t e x ~ j o m\end{array}$ & 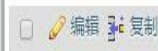 & 1) (2) 那余 234864 & $1989-08-01$ & 2 & HKS & 260 & 0.1 & NULL 1.4 & 25.8 & 24.0 & 23.1 & 85 & N.A. & N.A. 2014-09-03 15:38:16 \\
\hline t-3i) china_pollution & 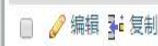 & 1) (2) 此除 234865 & 1989-08-01 & 3 & HKS & 260 & 0.3 & NULL 1.8 & 25.7 & 23.8 & 23.0 & 85 & N.A. & N.A. 2014-09-03 15:38:16 \\
\hline +1) contamex & 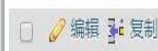 & 1) 那除 234866 & 1989-08-01 & 4 & HKS & 340 & 0.5 & NULL 2.4 & 25.6 & 23.4 & 22.3 & 82 & N.A. & N.A. 2014-09-03 15:38:16 \\
\hline $\begin{array}{l}\text { +-1) Daorje } \\
\text { +(-3) data_gong }\end{array}$ & 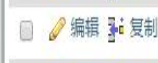 & 1) (2) 那除 234867 & 1989-08-01 & 5 & HKS & 300 & 1.3 & NULL 3.6 & 26.0 & 23.0 & 21.6 & 77 & N.A. & N.A. 2014-09-03 15:38:16 \\
\hline -OT hongkhong & 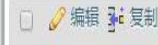 & 1) (-) 除 234868 & 1989-08-01 & 6 & HKS & 230 & 0.5 & NULL 3.2 & 25.4 & 23.2 & 22.1 & 82 & N.A. & N.A. 2014-09-03 15:38:16 \\
\hline $\begin{array}{l}\text {-圆新建 } \\
0 \text { daily meteo }\end{array}$ & 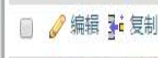 & (1) 朋除 234869 & 1989-08-01 & 7 & HKS & 20 & 1.0 & NULL 3.4 & 25.7 & 23.1 & 21.8 & 79 & N.A. & N.A. 2014-09-03 15:38:16 \\
\hline t-y daly_station & 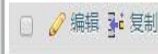 & 1) 正除 234870 & 1989-08-01 & 8 & HKS & 300 & 0.8 & NULL 2.8 & 26.1 & 23.2 & 21.8 & 77 & N.A. & N.A. 2014-09-03 15:38:16 \\
\hline$\Phi-h$ seg_station & 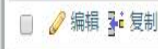 & (1) 那除 234871 & 1989-08-01 & 9 & HKS & 320 & 1.6 & NULL 4.7 & 26.7 & 23.4 & 21.8 & 75 & N.A. & N.A. 2014-09-03 15:38:16 \\
\hline
\end{tabular}

Figure 2-15: MySQL data base

\subsubsection{ElasticSearch}

Elasticsearch is a search engine based on Lucene, which provides a distributed, multitenant-capable full-text search engine with an HTTP web interface and schemafree JSON documents as shown in Figure2-16. Elasticsearch is developed in Java and is released as open source under the terms of the Apache License, and is the most popular enterprise search engine followed by Apache Solr, also based on Lucene.

\subsubsection{R}

$\mathrm{R}$ is a free software environment for statistical computing and graphics. In $\mathrm{R}$ community, a wide variety of packages based on various statistic methods and machine learning algorithms have been developed, and many news packages regarding new algorithms are still developing. The packages regarding majority algorithms in our 


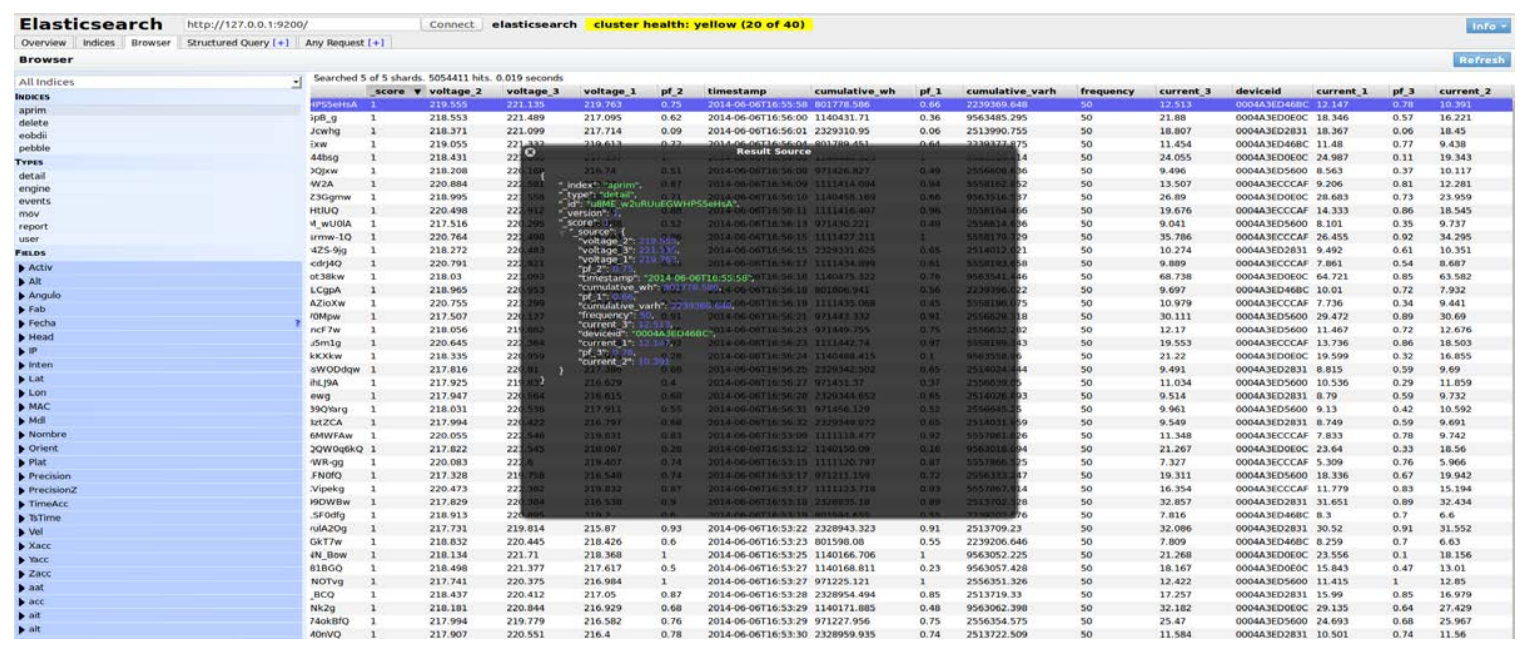

Figure 2-16: ElasticSearch data base

study can be found and installed for the corresponding model training. However, some algorithms employed are not available. Hence, the realization of these algorithms are needed.

Instead of treating $\mathrm{R}$ as a statistical software, it is better to say that $\mathrm{R}$ is an environment mathematical calculation. R provides more than some statistical procedures, users only need appoint specific database and parameters to carry out a statistical analysis. The idea of $\mathrm{R}$ is not only to provide some integrated statistical tools, but it is more to provide a variety of mathematical calculation, statistical computing function, so that users can process data flexibly, and even create a new statistical method to meet the need.

Table 2.3.3 lists some of the key packages that are necessary and installed in order to assist the whole process of the research including data retrieve, data manipulation, modeling, and visualization part etc.

\subsubsection{Python}

Python is an object-oriented program. For this study, it is mainly used for web scraping, that is, practicing of gather data through any mean other than a program interacting with an API. This process is commonly finished by writing an automated program that queries a web server(twitter post or Wikipedia pages, for instance), and 
Table 2.18: List of $\mathrm{R}$ Packages required

\begin{tabular}{|c|c|}
\hline Packages & Function \\
\hline RMySQL & Database Interface and 'MySQL' Driver for $\mathrm{R}$ \\
\hline DBI & R Database Interface \\
\hline dplyr & Data manipulation \\
\hline zOo & Regular and Irregular Time Series \\
\hline unbalanced & $\begin{array}{l}\text { This package implements some of most well-known techniques and propose a } \\
\text { racing algorithm to select adaptively the most appropriate strategy for a given } \\
\text { unbalanced task. }\end{array}$ \\
\hline caret & $\begin{array}{l}\text { A set of functions that attempt to streamline the process for creating predictive } \\
\text { models }\end{array}$ \\
\hline randomForest & Classification and regression based on a forest of trees using random inputs. \\
\hline e1071 & Support Vector Machine \\
\hline rpart & Classification and regression tree \\
\hline gbm & $\begin{array}{l}\text { An implementation of extensions to Freund and Schapire's AdaBoost algo- } \\
\text { rithm and Friedman's gradient boosting machine }\end{array}$ \\
\hline caretEnsemble & For creating ensembles of caret models \\
\hline som & Self-Organization Map \\
\hline neuralnet & Artificial Neural Network \\
\hline Kriging & Kriging algorithm \\
\hline doParallel & $\begin{array}{l}\text { Simple and highly optimized ordinary kriging algorithm to plot geographical } \\
\text { data }\end{array}$ \\
\hline ggplot2 & $\begin{array}{l}\text { An implementation of the grammar of graphics in R. It combines the ad- } \\
\text { vantages of both base and lattice graphics: conditioning and shared axes are } \\
\text { handled automatically, and you can still build up a plot step by step from } \\
\text { multiple data sources. It also implements a sophisticated multidimensional } \\
\text { conditioning system and a consistent interface to map data to aesthetic at- } \\
\text { tributes }\end{array}$ \\
\hline lattice & $\begin{array}{l}\text { A powerful and elegant high-level data visualization system inspired by Trellis } \\
\text { graphics, with an emphasis on multivariate data }\end{array}$ \\
\hline ggmap & $\begin{array}{l}\text { A collection of functions to visualize spatial data and models on top of static } \\
\text { maps from various online sources (e.g Google Maps and Stamen Maps). It } \\
\text { includes tools common to those tasks, including functions for geolocation and } \\
\text { routing. }\end{array}$ \\
\hline
\end{tabular}


than parse that data to extract needed information and expected formats [153] for the purpose of data analysis. For instance, in this study, the messages with user, time, location, and tweets with specific key words from Twitter can accessed and retrieved into json. file. The volumes of the tweets with specific keywords are used as inputs for the hospital admission forecasting while The tweets that express the individual' comments or emotions are for the sentiment analysis. Python script run based on 24 cores Linux system with processor model AMD Operaon 6234.

\subsubsection{Stata}

Stata is a data analysis and statistical software that created in 1985 by StatCorp. This software is a powerful tool and capable of data management, statistical analysis, graphics, simulations, regression. and custom programming. Therefore, it is wide used in the research society and in the fields of economics, sociology, political science, and biomedicine etc. Stata software on Windows 10 is only used for a basic statistic analysis, panel data analysis in this case, for the sake of its ease of use and clear results presentation. 


\section{Chapter 3}

\section{State of the Art}

In this chapter, a comprehensive and a holistic literature review regarding the air pollution modelling and energy analysis that is related to this monograph's interests will be conducted in this chapter. Concretely, it consists of the following list:

1 The methodology regarding air pollution network design and reconfiguration.

2 The methods regarding air pollution short-term forecasting.

3 The methods of identifying the sources and key factors that are related to air pollution in the local areas.

4 The outcome related air pollution, mainly focuses on the hospital admission.

5 The methods for pollution exposure exposure evaluation for the individuals.

6 The previous related regarding energy awareness systems.

7 The cluster methods and indicators for energy efficiency analysis.

\subsection{Monitoring station re-configuration}

A methodology for designing the optimum number of ambient air quality stations was presented in the study [13]. The fuzzy Analytic Hierarchy Process(FAHP) with 
triangular fuzzy numbers(TFNs) was used to identify the most desirable monitoring locations. The concept of sphere of influences determines(SOIs) the network configuration in the end. SOIs are dictated by a predetermined cutoff value in the spatial correlation coefficients between the pollution concentrations at the monitoring stations identified from the first step and the corresponding concentrations at neighboring locations in the region. The optimal station locations were determined by the rank of the utility scores. In this study, not only environmental factors are considered, some other factors like pollution, installation cast are also included for the scores evaluation.

Many studies see the air monitoring stations network design as optimization problem. For instance, an air monitoring network is optimized in the research[1]. A hybrid algorithm that combing generic algorithm(GA) and SA was conducted to minimize of the mean kriging variance derived from simulated values of a chemical transport model(CTM) instead of observations. In similar, [154] and [75] applied GA and SA respectively for $\mathrm{O}_{3}$ network with several hundred stations in the United States and France.

During the process of optimization, the crucial issue is how to design criteria for each regular grid cell of design domain where no observations are available for validation [11]. Three types of criteria are considered in the existing studies, that is, geo-statistic criteria and notion of entropy. The first one seek to minimize the theoretical extrapolation that related to the optimal experimental design theory[155]; The second one refers to information theory aiming to reduce the uncertainty given the network observations is maximized[156]; The last one is the physical criteria, which is to assess the ability of a network reconstruct a reference physical pollution field from the observations sampled at the network sites. The reference pollution field can be inferred from regional pollution simulation tool such as CTM[75]. The study [75] compared these three types of criteria combining with different simulation tools such as CMT and Kriging. It found that the optimal redistribution of ozone pollution network is sensitive to the criteria, not to algorithmic setting. The physical criteria was highly recommended for complicated design objectives, for instance, those related 
to the heterogeneity of the ozone field.

Another relevant study is from [14]. Instead of designing monitoring stations for air pollution, it focused on the groundwater quality monitoring. This research utilized a statistical learning algorithm named relevance vector machine(RVM) for obtaining solutions to regression and classification task. Monte Carlo(MC) simulation was adopted for capturing the uncertainties. MC modelling can provide several thousand realization of nitrate distribution in the aquifer. Subsets of these subsets are then used to design the monitoring stations, which is done by the process of finding the best-fit model of nitrate concentration distribution everywhere in the aquifer for each MC subset using RVM. The number and the location of monitoring wells were chosen based on the performance of RVM prediction.

Based on the review, it clear showed that the criteria selection is a crucial art for monitoring. Moreover, it is essential to utilize the simulation techniques for interpolating values into the grid with empty value. Metaheuristic algorithms are widely used for selecting the optimal monitoring network. Moreover, the availability of the spaces for the monitors are uncertain; The simulation by the statistic algorithm is inaccurate; The optimization process is with intensive computation. Under this situation, a new method for re-configuring the monitoring network based on the existing ones are proposed by a case study in Chapter 4

\subsection{Socio-economic activities factors: influencing on pollution}

Social economic activities are the main factors on the formation of pollution. Especially, with rapid urbanization and industrialization in the last century, pollution level have augmented significantly because of the human activities like manufacturing and transportation. Understanding and finding out the correlation between pollutants and social economic activities cannot only help the government enact specific regulation to reduce pollution level from the original sources, but also could benefit for the 
pollution forecasting by adding the relevant features.

A research from the World Bank showed that the scale of industrial activity, such as employment numbers and the number of establishments, and the process technologies activity etc. heavily affect industrial pollution[120]. The study [121] also indicated that industrial point sources and road network intersections are the main predictors for the pollution $\mathrm{NO}_{2}$ and VOCs in Ottawa, Ontario, Canada. High-level volatile organic compounds ( $\mathrm{VOCs}$ ), which is one of the precursors of $\mathrm{O}_{3}$, were found in the industrial district, followed by those in the commercial district, central business district and finally the residential district in Hong Kong[122].

Some studies about tunnels and road-side were conducted to show the relation between pollution and vehicle sources and they believed that the emissions from vehicles is one of the main contributors to the pollutants $P M_{2.5}$ and $P M_{10}[157$, 158. They figured out that the volume of traffic, the categories of vehicles, and meteorologic factors (wind speed and wind direction) are the main influencing factors in the formation of $P M$ in HK. Among all categories of vehicles, the ones that used diesel and gasoline engines mainly contributed to the formation of $P M_{2.5}$ and $P M_{10}$.

Moreover, the some relationships between $\mathrm{NO}_{x}, \mathrm{CO}$ and diesel-fueled vehicles were found respectively. A review from [159] summarized the input variables for land use regression model from six studies. They classified predictor variables into four categories: road type, traffic count, elevation and land cover, of which the traffic count on the nearest road is the most important factor for $P M_{2.5}[160,159]$.

Besides, the study [161] showed that the household activities, like cook and cleaning, which consumes energy, can also emit pollutants, such as total suspended particles(TSP), $\mathrm{NO}_{x}$, and $\mathrm{SO}_{2}$. In urban households, the gaseous fuel, coal and kerosene stove were the most used and generate pollution. It measured each pollutant emission in respect to fuel and stove types.

Construction, on the other hand, is considered as a main source to generate $P M_{2.5}$ due to the production of non-metallic mineral products in China[162, 163, 163] . Some researches also showed that construction process produce emission of $\mathrm{CO}_{2}$, $\mathrm{CO}, \mathrm{NO}_{x}, \mathrm{SO}_{2}$ and $\mathrm{PM}$ et al[164, 165]. A case study was conducted to assess the 
environmental performance of a building construction project in HK from cradle to the end of construction by using Environmental Model of Construction method, which aims to analyze the construction activities according to material, transport, energy consumption, and waste treatments, etc at each step. The results indicated that material is the major contributor to environmental impacts of the upstream stage of public housing construction and the carbon emission of the case studied project amounts to $637 \mathrm{~kg}$ carbon dioxide equivalent per square meter of the gross floor area[166].

Meteorological factors are of great importance for pollution formation. Ozone, notably, is affected significantly by the meteorological conditions, since wind speed and wind direction play an important role in the transporting of pollution from the Pearl River Delta to the Hong Kong area[157.

In summary, many of these previous studies revealed the correlation between emission sources and pollution from energy and material consumption perspective. The data of energy consuming of the possible emission sources are not available for some areas, including HK, the case in this study. They conducted questionnaires surveys or make sampling studies by measurement emission directly by advanced instruments from sampling sites to obtain the data. However, this needs high technical training process and many material resources. To address these issue, Chapter ?? provide a case study to illustrate how to identify the important factors for $\mathrm{O}_{3}$ pollutant by open source database and statistic analysis.

\subsection{Local short-term pollution forecasting}

Based on the forecasting scales, pollution forecasting can be categorized into two parts: local and regional. Both scales are considered by the fact that the long time residence of ozone concentration with the weather conditions, it enables long-range transport over hundreds of thousands of kilometers, which favors the ozone formation at both local and regional scales[108.

CMAQ shorted for Models-3 Community Multiscale Air Quality(CMAQ), is a 
widely used system for pollution simulation and forecasting in regional scale. Research [61] employed CMAQ with the process analysis tool to study the seasonal variations and formation mechanisms for major pollutants in China. The weather forecasting from Weather Research and Forecasting(WRF) as inputs for CMAQ shows a good modeling performance for 8-hour $\mathrm{O}_{3}$ prediction.

Unfortunately, the issue raised up since the simulation results are terabytes of data available for analysis against observed data which conventional data analysis are not well suited. Therefore, more efficient evaluation tools are required. The Meteorological Evaluation Tool for(AMET) evaluation Weather Research for CMAQ simulations is the product under such demands[167]. AMET use MySQL database to store and access a large amount of data, while $\mathrm{R}$ project is required for statistical computing and graphic production. Obviously, the whole modelling process involves many requirements and software installed. Moreover, the terabytes of data for building models also leads to a large amount of computation that is characterized time consuming.

Due to the reasons aforementioned, the regional models are not ideal tools for the short-term and real-time forecasting. Alternatively, the ability of AI techniques to learn from copious datasets and sniff out the patterns that are complex for human to detect, make them as attractive and effective tools for prediction, pattern discovery, function approximations etc.

By comparison with the MM5/CMAQ and ANNs, the study from [168] evaluates the efficiency and accuracy of both techniques for $P M_{10}$ concentration prediction. The computation time for 24-h CMAQ simulation was 5h using 4 nodes on a High Performance Computer(HPC) Linux Cluster and for ANNs was about 10 mins using a workstation SUN Blade 1500, 1.062GHz UltraSPARC III, 2GB RAM, Sun Solaris. The better prediction was found for ANNs rather than CMAQ. Consequently, ANNs was recommended as a superior competitor to photochemical models for practical air quality prediction.

The starting time of AI application in the field of local short-term pollution prediction should be the early 1990s. Afterwards, a number of literature have generated 
remarkably. In the following subsections, a comprehensive and holistic reviews regarding the pollution forecasting models with aspects of feature selection and AI techniques, are presented.

\subsubsection{Features selection techniques}

Before modelling by AIs, the construction and selection of features from the raw data sources should be given more focus as emphasized in [56], since the more the input features possess correlated behavior with the dependent variable, the easier the learning process is for the algorithm and the better forecasting results can be obtained. Conversely, more redundant or irrelevant features not only increase the noise and computational time, but also probably degrade the performance of the models [57].

As emphasized from [57], if many features that are not necessary for prediction the desired output, it could be deleterious to the prediction accuracy in practical machine learning, and lead to more computation time. Feature selection is a very useful data

mining technique that reduces the effects of irrelevant features with the data. The reduced dataset with fewer features can easily handle, reduce the performing time, and defy the curse of dimensionality to improve prediction performance [169, 170]. A lot of state of the art methods about feature selection has been proposed, such as principle component analysis, genetic algorithm, self-organizing maps, random forest [171, [172].

Both studies [173, 56] state the importance of input selection when developing of neural networks for the prediction in the environment application. The relevant research regarding feature selection in environment modelling application is from [108], where principal components are from raw features as new inputs. Feed-forward ANN varying structures and multiple linear regression was adopted to predict ozone concentrations. The results showed that the principal components may not improve modeling accuracy. However, its application led to the introduction of fewer variables and thus reduced the complexity of modeling and eliminated data col-linearity without losing ANN models performances. 
Research from [149] applied feature selection and machine learning methods on urban environments noise pollution prediction. In this work, the feature selection techniques, namely generic algorithms and wrapper feature selection, were employed. The results compares to the the classical data reduction techniques principle component analysis, showing that theses two feature selection techniques can reduce the complexity of modeling without compromising modelling accuracy.

\subsubsection{Multiple Linear Regression(MLR)}

Multiple Linear Regression(MLR) is an approach to modeling the relationship between dependent variable and one or more explanatory variables. The case of one explanatory variable is called linear regression[30]. For more than one explanatory variable, it is called multiple linear regression.

Similar to other prediction problems in the world, the relationship is hard to be linear between pollution and its influencing factors in the environment domain. The scholars always have envisioned that MLR could not perform as well as other AI techniques. Accordingly, MLR frequently is utilized for the purpose of comparison to the more sophisticated AI techniques, such as artificial neural network and support vector machine [174, 108, 175].

For instance, the study from [16] utilized ANN to forecast ozone pollution, compared to traditional MLR models. Specifically, MLR models and ANN are examined for a range of cities under different climate and ozone regimes, enabling a statistical comparative study of the two approaches. The results indicate that neural network techniques are somehow (but not dramatically) better than regression models for daily ozone prediction. Moreover, all types of models are sensitive to different weather-ozone regimes and the role of persistence in aiding predictions.

In addition to the limitation of capturing non-linear capability, col-linearity of input variables is a key factor that infect the MLR models' performances [108, 176]. Because of that, MLR models always were employed after transformation of inputs by feature selection or feature reduction methodologies,such as Principle Component Analysis(PCA), to eliminate the collinearity and improve the forecasting performance 
[97, 177, 108, 106, 178].

\subsubsection{Time Series(TS)}

Time series is an auto-regression models, that is, the output is related to the values in the past of itself. It has been an popular method employed in the environmental application in between 1970 and 1990. However, with the advent of development of machine learning techniques such as ANN and SVM, TS, like MLR, is used for comparison with other these techniques as well.

The relevant study from [108] employed MLR, time series and feed-forward ANN models for daily ozone concentrations prediction. The results showed that time-series modelling was not profitable. Nevertheless, similar performances were obtained in either case: multiple linear regression and feed-forward artificial neural network.

By comparing with two traditional statistical models-linear regression and BoxJenkins ARIMA, which is one type of TS. The fitting of ANN model is superior than the traditional ones predicting Houston's daily maximum ozone concentrations [179]

Similarly, research [180] used BoxJenkins (ARIMA) and MLR models to forecast air quality in urban areas, but with limited accuracy owing to their inability to predict extreme events. In contrast, ANN can recognize non-linear patterns that include extremes. A novel hybrid model combining ARIMA and ANN to improve forecast accuracy for an area with air quality and meteorological data was applied to Temuco, Chile, where residential wood burning is a major pollution source during cold winters. Experimental results indicated that the hybrid model can be an effective tool to improve $P M_{10}$ forecasting accuracy obtained by either of the models used separately.

Another study [181] forecast daily averaged concentrations of respirable suspended particulate matter(RSPM) by three models - MLR, Box-Jenkin ARIMA, and a combination of ARIMA and MLR by meteorological factors. The result illustrates that the proposed hybrid model is recommended as it gives better forecasting performance in comparison to the other two separated models.

Above all, pollution-weather relationship is complex and nonlinear, especially for 
the pollutant like ozone. The regression models might not be captured the characteristics of such pollutant. Therefore, the AI techniques, of which artificial neural network, support vector machine, classification and regression trees gain substantial attentions and have been evidenced their effectiveness on short-term forecasting in many fields.

\subsubsection{Artificial Neural Network(ANN)}

Not surprisingly, ANN is one of the most popular AI techniques that have been applied in the environment application, not only because of its effectiveness of prediction but also its various structures and configurations. For instance, the study [182] tested 216 structures of ANN models to forecasting $\mathrm{NO}_{2}, \mathrm{SO}_{2}, \mathrm{O}_{3}$. Moreover, Generic Algorithm(GA) was designed to select the most relative inputs for these models.

As shown in Chapter 2, ANN can be categorized into different groups based on various criteria. Among various of structures, MultiLayer Percetron(MLP) and Radial Basis Function (RBF)that belongs to feed-forward back-propagation ANN are the most widely adopted.

Three feed-forward back-propagation ANNs were trained using historical data in the research from Abudul-Wahab[183]. The main emphasis of the first model has been placed on studying the factors that control the ozone concentrations during a 24-hour period (daylight and night hours were included). The second model was developed to study the factors that regulate the ozone concentrations during daylight hours at which higher concentrations of ozone were recorded. The third model was developed to predict daily maximum ozone levels. The results of this study indicated that the artificial neural network (ANN) is a promising method for air pollution modelling.

The study [184] applied ANN with several structures, TS, and MLR models to predict ozone concentration in Oporto city. TS model was not profitable. Better performance indexes were achieved by feed-forward ANN with twelve neurons input layer, ten neurons in hidden layer, and one neuron in the output layer. Considering the learning speed, the accuracy and generalization ability etc. all the relevant parameters, the study [26] chose the feed-forward ANN with RBF for predicting max- 
imum ozone level in Hong Kong and used adaptive RBF methodology to determine the numbers nodes in hidden layer. Similarly, the study [185] employed another ANN topology-MLP, and MLR models to forecast ozone and nitrogen dioxide levels in real-time to $8 \mathrm{~h}$ head in Bilbao(Spain). The performance for MLP are highly recommended.

The structures of MLP and ELMAN networks was used for predicting the maximum tropospheric ozone concentrations for the next day in Mexicali-Calexico border data in the study[30]. Meanwhile, the more techniques like persistence parametric prediction, semi-parametric ridge regression, Support Vector Machine(SVM) are involved in comparison with the two ANNs. The results clearly showed that the ANNs, specially MLP, displayed a prediction capacity with better performance than others, followed by SVM, which has a slight worse performance than ELMAN neural network. The performances achieved by the Linear model, Ridge Regression and the Persistence model were notably worse, as expected due to the highly non-linear and disperse behavior of the data.

The research [93] classified models into two regression type models(MLR, ANN), and Gene Expression Programming and Auto-regression-type models(Nonlinear Local Prediction and Auto Regressive Integrated Moving Average) for developing a predictive ozone model in Tabriz. The results of regression type models are not particularly promising for establishing a forecasting capability comparing with the Auto Regressive type ones.

Moreover, in order to obtain more efficient and accurate models, some studies preferred to adopt some techniques for input transformation before model training. For example, principle component analysis(PCA) is the most common tool for input transformation. The study [175] aimed to predict tropospheric ozone concentration in the area of Turkey by MLR model. It extracted the most important components from inputs, in which way the input dimensions can be successful reduced. The main components were, then, used as new inputs for MLP model with two hidden layers with fifteen neurons. In another case [186], four models, that is, two-stage ANN, MLR, two-level clustering followed by MLR, and MLP , were established by using 
meteorological conditions data to predict ozone concentrations. The two-stage neural network aforementioned utilized a self-organization map(SOM) followed by K-means to cluster meteorological condition into different meteorological regimes, in each of which MLP was used to simulate the nonlinear ozone-meteorology relationship. The results showed that this proposed two-stage networks was suggested with the best prediction performance among the four models.

However, these studies mainly limited to build various structures of models, and lack of a step framework with details regarding requirements of building ANN, for instance, selecting input variables, choosing the key network parameters. Consequently, it lacks procedures of ANN development, which will lead to the experiment hardly repeatable and reproducible, and quality of the ANN models. Nevertheless, repeatability of experiments and reproducibility of foundings are of importance for the contribution in the intended field. In this regard, the study from [56] proposes a systematic protocol for development and documentation of ANN models in the application of environment. It emphasized that not only selection of model architecture is of importance in the ANN modelling, the relevant feature selection is also a key step. This also main reason drives us to consider feature selection as a vital aspect to be studied as mentioned in this study.

Time series was employed for average monthly ozone prediction over Arosa, Switzerland [17]. First of all, the total ozone time series has been identified as a complex system and then ANN models in the form of MLP with back propagation learning have been developed. The models are Single hidden layer and two hidden layers perceptrons with sigmoid activation function. After sequential learning with a learning rate of 0.9 the peak total ozone period (FebruaryMay) concentrations of mean monthly total ozone have been predicted by the two neural net models. After training and validation, both of the models are found to be skillful. But the two hidden layer perceptron is found to be more adroit in predicting the mean monthly total ozone concentrations over the aforesaid period.

However, one of the shortages of $\mathrm{ANN}$ is that it is easy to stuck in the local minimum. Some optimization techniques, for instance, generic algorithm(GA), were 
always employed for addressing this problem. On the other hand, it drives other techniques such as SVM to be a better alternative tool to avoid the local minimum problem. For the latter one, a comprehensive literature review is presented in the next subsection.

\subsubsection{Support Vector Machine(SVM)}

SVM is another powerful prediction technique. Compared to ANN, it has some advantages like global optimum,good generalization ability and dimensional independence 129, [187], therefore SVM are draw a lot of attentions and were developed successfully in many cases in recently years. [188, 184, 30, 31, 129, 32, 33, 189, 31, 190.

In machine learning, support vector machines(SVM) are supervised learning models with associated learning algorithms that analyze data and recognize patterns, used for classification and regression analysis. The original SVM algorithm was invented by Vladimir N. Vapnik and the current standard incarnation (soft margin) was proposed by Vapnik and Corinna Cortes in 1995[191].

Research from [192] used SVM with kernel functions, radial basis, Polynomial and Spline function to predict $\mathrm{O}_{3}, N O_{3}$ and $P M_{10}$ respectively in Mexico City. The experiment results show that the kernel radial basis function is more stable and accurate than others, although sometimes it increases the computational cost. Polynomial kernel does not offer an adequate performance in comparison with radial basis function

SVM was carried out for prediction of hourly ozone values in Madrid urban area from [193]. Various configuration of input variables were prepared for testing the influencing factors, such as the previous ozone measurements, measurements in neighbor stations, and the meteorological factors. By the statistical comparison with MLP, the SVM model obtained much better mean accuracy. The optimal prediction is 4 hours ahead. Moreover, the meteorological variables, solar radiation and temperature, that can significantly enhanced the accuracy of the accuracy of the models, are identified as important variable in the study.

$\mathrm{O}_{3}$ prediction in Beijing was conducted in the study [32]. The records of meteoro- 
logical factors, temperature, humidity, wind velocity and UV radiations was selected as inputs for forecasting. SVM was used for classifying the data into its corresponding categories,then Back Propagation ANN with genetic algorithm was utilized to forecast ozone concentration in Beijing. High performance of model fitting was achieved using those combination method.

SVM models are a close cousin to classical multi-layer perceptrons ANN. Using a kernel function, SVM is an alternative training method for polynomial, radial basis function and multi-layer perceptrons classifiers. The weights of the network are found by solving a quadratic programming problem with linear constraints, rather than by solving a non-convex, unconstrained minimization problem as in standard neural network training[194]. According the research results and considering both computational cost and accuracy, this paper prefers SVM with kernel radial basis function to predict maximum $\mathrm{O}_{3}$ level.

In the study of [195], the advantages of SVM techniques were over ANN in the aspect of generalization ability when there are relatively small numbers of learning data and large of input features.It is found that the SVM regression technique has been used for nding approximate solution of the complex nonlinear problem consists on modeling the air quality in Oviedo urban area at local scale with success.

A another comparison with ANN was also carried out. But the result of OrtizGarcas research, contrary to Salazar-Ruizs, demonstrated that SVM regression could provide a better predictor than Multilayer Perceptron. That is because in the OrtizGarcas case, when the database showed a non-linear characteristic, Multilayer Perceptron gave worse learning capability than Support Vector Machine for Regression Algorithm[175].

\subsubsection{Classification and Regression Trees(CART)}

Because of some difficulties such as the vulnerable stability of tree, CART was not widely used as much as ANN and SVM, but still got some attention from the researchers, since its faster computation and characteristics suitable for feature selection. C4.5 and random forest of which are the two main algorithms [196]. 
The introduction of random forests proper was first made in a paper by Leo Breiman [132]. This paper describes a method of building a forest of uncorrelated trees using a CART like procedure, combined with randomized node optimization and bagging. In addition, this paper combines several ingredients, some previously known and some novel, which form the basis of the modern practice of random forests, in particular: using out-of-bag error as an estimate of the generalization error, and measuring variable importance through permutation. The report also offers the first theoretical result for random forests in the form of a bound on the generalization error, which depends on the strength of the trees in the forest and their correlation.

In the study of [197], the researcher helped Texas Commission on Environmental Quality(TCEQ) to build a highly accurate ozone level alarm forecasting models for the Houston area, they chose bagging probabilistic decision trees and random forest for prediction. The results shows that 10\% higher in precision than other sophisticated artificial intelligent techniques such as SVM used in this particular case.

Combining the CART, ANN and MLR with principle component analysis, the study [123] forecast $P M_{10}$ concentration in Thessaloniki,Greek, showing that all of the techniques can capture the $P M_{10}$ concentration trends. In terms of performance measurement- index of agreement, CART performed better than others.

[64] used Single Decision Tree(SDT), RF, and decision treeboost(TB) that combines the strengths of regression tree and boosting algorithm, to predict urban air quality, and compare with SVM approach as benchmark. The result demonstrated that DTF and RF models outperform the SVM both in classification and regression which could be the attributed to the incorporation of bagging and boosting algorithm.

\subsubsection{Ensemble Models}

Ensemble models were widely applied in regional-scale ozone concentration prediction. Air Quality Model Evaluation International Initiative(AQMEII) shared the webdistributed ENSEMBLE system to all the groups participated making model intercomparsion and multi-ensemble analysis conveniently. The 10 individual regionalscale models provided by different research group including CMAQ,WRF,EMEP 
et.listed in the study of [198]. This initiative was based on the evidence that the combination of outcomes of predicted by several models as a new ensemble model has already tested to improve the model performance comparing with individual model in many studies [198, 199].

In the study of [199], the ensemble model combining EMEP, European Air Pollution Dispersion(EURAD)model, the Long-Term Ozone Simulation(LOTOS) Model and Regional Eulerian Model with three different chemistry schemes(REM3) performed a superior performance than any individual performance for ozone prediction.More than $10 \%$ of root-mean error decreases by using Least squares methods for combining 48 protential models when predict regional-scale $\mathrm{O}_{3}$ concentration. [199].The ensemble based on the mean of the seven models including CMAQ,WRF/Chem et. and the ensemble based on the median are found to have significantly more temporal correlation to the observed daily maximum 1-hour average and maximum 8-hour average $\mathrm{O}_{3}$ concentrations than any individual model[200].

According to the summary of local prediction studies in the section above,each model is not perfect. Instead of examine the causes of model bias,transfer the ensemble techniques applied on regional-scale case to the local-scale ones could be a good idea to mutual cancel the error of individual models for getting a better promising one.

Ensemble methods that improve the prediction performance that outperforms those of single models are widely used nowadays. Usually, there are two phases:1)the production of multiple predictive models; 2) their combination [201].Linear regression ensemble and greedy ensemble are two main types of ensemble methods.

Linear Regression Ensemble is the simplest ensemble method. It is like the MLR method, but the difference between them is that instead of the independent value of the variable, Linear Regression Ensemble uses the individual model members(regressors), which have been trained before. The mathematical equation can be given as following:

Greedy ensemble look for the globally best subset of regressors by making local greedy decisions for changing the current subset[201]. There are two different groups 
of greedy ensemble algorithms: forward selection and backward elimination. Figure 6 gives a picture about these two greedy ensemble algorithms. $h_{t}(\mathrm{t}=1,2, \mathrm{n})$ are the sets of individual regression model members that constitute the ensemble.

\subsubsection{Unbalance Techniques}

A problem arises when forecasting the occurrence of ozone episode days (exceedances of limitations) in many studies, leading to an imbalanced dataset classification problem. The study from [35] affirms that the standard ANN methods cannot make accurate prediction of the exceedances of daily maximum $8 \mathrm{~h} \mathrm{O}_{3}$ concentrations. Two ways of cost-sensitive algorithms, cost-penalty and moving threshold, were employed for addressing the problem.

Another relevant study [71] proposed a cost-sensitive for the SVM to predict ground-level $\mathrm{O}_{3}$. in Hong Kong. The experiments show that cost-sensitive SVM can avoid class imbalance dataset problem with lower percentage of false negative on exceedances of limitation days but with higher false positive on un-exceedances days. This research also used regression-classification mode, that is using support vector regression model to predict the numerical value, after converting its output to binary one., compared with the classification mode. Finally, the cost sensitive with SVM classification mode was recommended to forecast $\mathrm{O}_{3}$.

Few studies put efforts on the pollution forecasting with imbalance dataset issues. However, since unbalanced data issue has been found in many application areas, this problem has been obtained attraction from the scholars in the discipline of machine learning. To address it, the algorithms far more than cost-sensitive have been modified or developed, which can be categorized into three parts [150]:

- Data level: Re-balanced two class dataset before modeling. Two main ways are involved in this level: over-sampling and under-sampling. Over-sampling aims to increase the samples of minority class. In contrast, under-sampling is to reduce the instances in the majority class.

- Algorithmic level: Algorithmic level ways focus on carrying out modification on 
existing algorithm to strengthen their ability of learning from minority class. In this category, cost-sensitive is included.

- New evaluation metrics: Instead of using traditional evaluation metrics as criteria for algorithm's object function, the new performance metric adapted into imbalanced data problem such as Receiver Operation Characteristics(ROC), Gmeans, and F-values are less likely suffer from imbalanced distribution because that have less bias towards majority class.

Based on the above comments, it is fully clear that the local short-term pollution prediction can be improved by many ways. For instance, employing various feature selection algorithms is an effective way to reduce the complexity of modelling without compromising the prediction accuracy. The ensemble algorithm has replaced the single algorithms playing a crucial role in enhancing modelling performance in the data mining area. In addition, utilizing unbalanced techniques is necessary for tracking the issue of failing to predict high-level pollution values. Therefore, it is worthy to discuss the utilization of these techniques. These techniques are tested and proved their effectiveness in the application of prediction pollution in Chapter 4 and Chapter 5 .

\subsection{Health evaluation personalization}

With the increasing development of Internet of things (IoT), the integration of sensing and embedded everyday computing devices at the edge of the internet will result in the evolution of embedded internet or the internet of things. Typical IoT devices includes physical items embedded with sensors, scissors with integrated circuit (IC) tags, and smart meters to remotely monitor energy consumption. The evolution of the IoT are consumer-centric mobile sensing and computing devices, which are connected to the Internet, these include smart phones, in-vehicle sensing devices and so on. They are typically equipped with various sensing faculties and wireless capabilities that allow them to produce data and upload the data to the Internet. 
In the environment domain, some person monitoring devices have been used to study exposure from air pollution. However, the characteristics of devices including the expensive and heavy are the main factors that impede the individual carrying outside and market penetration. Hasenfratz [202] proposed a mobile measurement system for the city of Zurich to derive accurate ultra-fine particles pollution (UFPs) maps with high spatio-temporal resolution. Their system collected a very large scale dataset of over 50 million UFPs measurement using mobile sensing nodes over more than two years. The mobile measurement system consists of ten sensor nodes installed on top of public transport vehicles. However, this comes at a cost of a reduced temporal resolution at any covered location, making it a significant challenge to derive pollution maps with a high temporal resolution at daily or hourly time scales. The authors developed land hourly time scales. The authors developed land-use regression (LUR) models to produce accurate pollution maps with high spatio-temporal resolution. Their LUR model used a set of explanatory variables based on Generalized Additive Models (GAMs) to model pollution concentrations at locations not covered by the mobile sensor nodes. The authors also evaluated the dependencies between the explanatory variables and the measurements, and exploited these spatio-temporal relationships to predict the pollution levels for all location without measurements but with available land-use and traffic information.

Under this condition, some studies aims to design and provide a low-cost and portable pollution measurement for tracking the pollution levels. For instance, study [203] designed a small and portable ozone measurement device for participatory air quality monitoring. This device is characterized low-cost and low power measurement named GasMobile. It connects a small-size ozone sensor to an off-the-shelf smartphone running the Android OS. In detail, they use external receptor and relevant corollary devices (e.g., USB host mode, Power supply in host mode and Power consumption) to take the measurement. In the part of software architecture, they choose the popular Cyanogen Mod custom kernel and android-serial-api for the serial communication between ozone sensor and smart phone. Davids model provides a high data accuracy by exploiting sensor readings near static measurement stations to 
regularly keep sensor calibration up to date. Finally they show that it is possible to increase public awareness and to create spatially fine-grained air pollution maps.

Another similar study is from 204], it identified a clear trend towards GPS based real-time tracking of individual time-activity patterns for personal exposure assessment, but also the need of new technologies for the design of future studies. In this study, they use a professional air quality monitor called Dylos to get the data on air pollution. Dylos air pollution monitor is trapped onto the backpack with the inlet and fan exposed to the air. The GPS is placed in the side mesh pocket. Dylos was used to measure PNCs which subsequently transformed into $\mathrm{PM}_{2.5}$ concentration. A GPS was used in combination with the Dylos to relate observe particle concentrations to time and location. They use contextual and time-based activity data to define six micro-environments(MEs)to assess everyday exposure of individuals to short-term $\mathrm{PM}_{2.5}$ concentrations. A low-cost particle counter based on light-scattering technology was combined with a GPS receiver to track movement and exposure of individuals across the MEs. Taking into account the information gained through personal exposure measurement, this work demonstrates the added value of data generated by the application of low-cost monitors. Efforts to understand the health effects of air pollution under direct exposure measurement are often infeasible because of high costs and low compliance 205]. Instead, indirect measurements are used by estimating an individual air pollution exposure level like what I mentioned above. However, the accuracy of such methodologies has been limited by the often untrue assumptions, such as people spend $100 \%$ of their time at home and that people living within the same area or distance from air pollution sources have similar exposure 206]. From the researches mentioned above, we can know many ways to predict and assess the air condition. However, they have more or less some inconveniences.

Nevertheless, majority people are not willing to take any addition sensors with them in their daily life except their own smartphone that has been an inseparable part for people's life. Moreover, the air quality monitoring stations designed by the authorities can provide adequate pollution information, and can be used for the personal pollution exposure evaluation by combining the personal information from 
smartphone. The evaluation based on existing air quality monitoring stations requires two conditions: personal tracking data and interpolating pollution value into the place where the fixed monitoring stations are not located.

Nicholas [207] argues that in the modern life, smart phones not only serves as the crucial computing and communication mobile device of choice, but it also comes with a rich set of embedded sensors, such as accelerometer, digital compass, gyroscope, GPS, which could provide an opportunity for selecting data at individual level and can be used for the health exposure of individual.

Glasgow 205] tested the use of smart phone technology to collect personal level time-activity data to be related with air pollution exposure. Because of the spatiotemporal variability of people and air pollution within cities, it is important to account for a persons movements over time when estimating personal air pollution exposure. In Glasgows study, they aimed to examine the feasibility of using smart phones to collect personal-level time-activity data. Using Skyhook wirelesss hybrid geo-location module, they developed a smart phone application designed to track participants location in 5-min intervals for 3 months. From 42 participants, they compared application data with contemporaneous data from two self-reported diaries. This studys high compliance and feasibility for data collection demonstrates the potential for integrating smart phone-based time-activity data into long-term and large-scale air pollution exposure studies. However, Glasgow uses Skyhook to collect indoor and outdoor air pollution data, but there is not often an abundance of Wi-Fi signal during the outdoor activities. Although efforts to improve the accuracy of indirect air pollution exposure assessment have focused on incorporating air pollution monitor data with individuals location to increase the accuracy of the data, what they have done are not enough.

A relevant study from [208] proposed a pilot study that applied momentary location tracking services supplied by smart phones, to identify an individual's location in space time for three consecutive months. The traffic related pollutant, $\mathrm{NO}_{x}$, was estimated by an annual mean $\mathrm{NO}_{x}$ surface modeled using the LUR modeling technique. Both stationary (including home, work and other stationary locations) and in-transit 
(including commute and other type travel) locations are included in the individual exposures estimation. The results shows that integrating momentary location tracking services with fixed-site field monitoring, available Wi-Fi networks indoor, plus indoor-outdoor air exchange calibration, makes exposure assessment of a very large population over an extended time period feasible. Inverse distance weighting (IDW) and Kriging [209], and land use regression(LUR)[210], spatiotemporal models such as Bayesian Maximum Entroy [211, 212] and dispersion models[213], are usually the tools to drive air pollution surfaces so subject within a study region can be assigned an exposure estimate for health outcome assessment [208]. In Su's study, Land Use Regression modeling of concentrations of traffic-related air pollution was conducted through a machine learning approach called Deletion/Substitution/Addition. Although LUR is a very useful method for researchers to make the spatial interpolation, many traffic information such as land roads have to be included, which will increase the extra work. Moreover, the prediction of $\mathrm{NO}_{x}$ in annual mean unit, which is not consisted to the daily individual movement data, is limitation for the study, since the levels of pollution concentrations variations based on times of day, seasons etc.

As discussed before, the air pollution exposure assessment concentrates on a group of people. Moreover, with the increase demands of high quality live, customizing the health evaluation for the individuals with smart sensors are necessary to meet these demands and has already been implemented by many researchers as aforementioned literatures. However, the high cost and inconvenience are one of the main obstacles for recording pollution outdoor by mobile devices. Indoor pollution monitoring by the smart sensors, on the other hand, can be installed without moving them. Moreover, for the people like children, elderly and workers in the office spend most of their time in the indoor environment, which should not be negative. Therefore, Chapter ?? of this monograph will promote a pilot study focus on the individual air pollution exposure estimation by the data gathering from the indoor smart sensor and public data from air pollution monitoring stations, as well as statistic and data mining techniques for modelling. 


\subsection{Energy used awareness in production processes}

Increasing the awareness of energy used during production processes involves a core phase to achieve the goals of energy management systems [86, 219]. In this regard, Weinert et al. [55] developed the EnergyBlocks methodology to predict the energy consumption of production processes. The methodology is based on the representation of production operations as segments of specific energy consumption for each operating state of the production equipment. Moreover, Seow and Rahimifard [220] implement a modeling approach to demonstrate manufacturing system energy flows that uses a product lens and builds on energy consumption data that were collected during the plant and process stages. This approach facilitates the itemization of energy used during production. In addition, Thiede et al. [221] proposed an energy portfolio for classifying energy consumers (e.g., machines) at manufacturing companies, and the derivation of target-oriented action plans to improve energy efficiency. In addition, based on analyzing the energy consumption and operational data of machine tools and manufacturing equipment, Vijayaraghavan and Dornfeld [80] introduced a framework to promote energy awareness at the production level. This enables decision making to improve the environmental performance of manufacturing systems. Nevertheless, they have simulated energy profiles based on available data of machine tools without including a real-time implementation of an actual factory production line. In fact, the focus on the operational level and the lack of energy information at the production level constitute an obstacle to providing valuable information to customers.

\subsubsection{Enhancing a sustainable production process by increas- ing energy awareness}

One means of achieving a sustainable process is to calculate the amount of energy used during the production process. In this regard, Faulkner and Badurdeen [1] presented a simple visual representation of the estimated energy consumption of the

production processes using sustainable value stream mapping (VSM), as shown in 


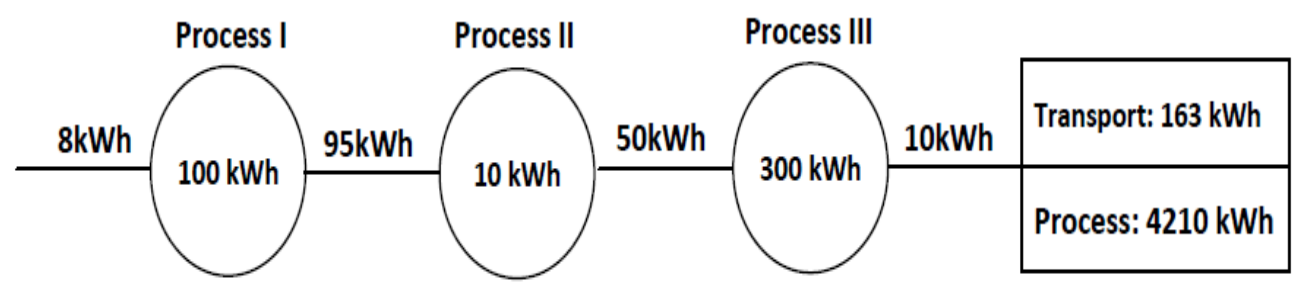

Figure 3-1: representation of energy consumption of a sustainable-VSM [1]

Figure 3-1. This VSM considers the energy used in each process and in the logistics between processes. The proposed energy awareness method that is presented in this paper enables lean manufacturing to present such a VSM based on accurate data.

Brown et al. 222] also mentioned the identification by sustainable-VSM facilities of environmental metrics using symbols that represent energy, water, and material waste in manufacturing systems. For this reason, the energy use of each process and in the transportation of the work material to and from the process is recorded. In fact, the benefits of the availability of energy data (at process or machine levels) are highly dependent on integrating the energy data with other data sources such as production data. Several studies highlight the need for such integration of various information flows [85, 76, 77]. Factory Energy Solution provides an example of such integration. It consists of a set of hardware and software solutions for energy management that is offered by Mitsubishi Electric [223]. It achieves energy savings by integrating and managing production and energy information to identify wasted energy in the production process. Moreover, Kurdve et al. 82 introduce research that considers the integration of production system models with environmental management. The researchers conclude that the lack of integration strategy and sustainability metrics, and the adaptation of improvement methods to push companies' operational performance, are major barriers to the expansion of such integration. 


\subsubsection{Technological requirements for energy awareness}

Due to the limitation of continuous measurements of energy consumption in conventional energy management, the energy consumption at process level cannot be obtained. This leads to a lack of insight on energy consumption [77], as identifying the energy consumption in the production process is the cornerstone to initial effective measurements to increase energy efficiency. Therefore, collecting real-time energy consumption data at machine level with smart meters based on the Internet of Things (IoT) paradigm is considered to be essential for increasing energy efficiency by enhancing the awareness of the energy consumption of production processes [224, 225]. It leads to the discovery of cost-saving opportunities and enhances energy-efficient production management practices and decisions at the production level.

Vikhorev et al.[77] indicated how the availability of real-time energy data at the process level helps in energy-efficient decision making at strategic and operational levels. Furthermore, they illustrated the core elements of an advanced energy management framework, including data standard requirements, data streams analysis and energy performance evaluation indicators. Nevertheless, this work is lacking at the production level. Specifically, it fails to link energy usage to production data.

Furthermore, Sequeira et al. [226] provided conceptual architecture for a cloudnative industry energy management systems solution with cloud computing capabilities. They emphasized that an energy management system for factories should have realtime, cross-site energy consumption monitoring that is integrated with energy and machine data, and energy data analysis.

In the industry 4.0 revolution, the factory of the future will symbiotically share material and energy flows with industry [227]. Here, IoT technology will be one of the key elements of smart factories in the new revolution industry [228]. IoT technology enables the exchange of information between different devices in real time. Such data could represent production status or energy consumption behavior.

In order to outline detailed energy flows, Zampou et al. [229] identified the main functionalities that are required to characterize an energy-aware information system 
in manufacturing. They grouped the functionalities into two categories: energy monitoring and energy-aware analytics. Their approach depends on integrating operational and energy information flow to accomplish the functions. The main limitation of this work is that they do not illustrate how energy and production process related data can be collected and linked automatically.

\subsection{Energy-efficiency pattern analysis}

Study from [88] explored the regional characteristics of inter-provincial $\mathrm{CO}_{2}$ emissions and the regional distribution of the reduction of emission intensity reduction in China by K-means, Hierarchical clusters, and PSO-FCM clustering algorithms which is a method using the Particle Swarm Optimization(PSO) on Fuzzy-C means (FCM). To be different from the previous traditional studies that divided China based on regions: eastern, central, and western, the research from [88] examined how the regions corresponding to the divisions by economic developing level. Moreover, five indices including total emission, per capital emissions, $\mathrm{CO}_{2}$ emission per GDP (intensity of $\mathrm{CO}_{2}$ emission), $\mathrm{CO}_{2}$ emission per unit of energy and the proportion of industrial $\mathrm{CO}_{2}$ emission are considered to characterize $\mathrm{CO}_{2}$ emissions in China.

Research from [89] analyzed China-s city level $\mathrm{CO}_{2}$ emission of energy consumptions by DMSP/OLS nighttime light imageries and explored major driving forces for proposing feasible mitigation policies. A recommendation is given to reduce the growth rate of $\mathrm{CO}_{2}$ without compromising economic development by optimizing the industrial structure of technology intensive areas and improve energy efficiency in other areas.

The study [90], one the other hand, compares the transport development and energy use with a focus on $\mathrm{CO}_{2}$ emissions in the EU15 countries. Three terms related energy and emission was conducted, that is, immaterialisation, dematerialization, and decarbonisation. Immaterialisation refers to the reduction of energy intensity and transport intensity production and can be measured by indicator energy intensity (energy/GDP); Dematerialization was defined as the reduction of carbon intensity 
of energy production and the carbon intensity of transport $\left(\mathrm{CO}_{2} /\right.$ energy $)$; Decarbonisation is the reduction in carbon of the whole economy, and it can be seen as a linkbetween immaterialisation and dematerialization, which is measured by carbon intensity of economy $\left(\mathrm{CO}_{2} / \mathrm{GDP}\right)$. This study groups EU15 countries based on each of three indicators aforementioned in order to establish different patterns. The limitation of this study is that it lacks of statistical analysis for identifying the main influential factors in each group.

A special IPAT identify case revealing the importance of distinct components or factors in the historical data of energy related $\mathrm{CO}_{2}$ emission, which is often called KAKY identity, have been widely utilized in many studies [91, 92]. Research [91] decomposes urban emissions into four components(emission from primary, secondary, tertiary industry, and residential areas) to obtain a result of the interacting among 13 macro influential factors. Thirty provinces in China was classified based on the historical data of the 13 macro factors. Shapley value decomposition was finally adopted to identify the key factors, in light of which the related policy control was promoted.

Finally, it is clear illustrated that the main limitation is either lacking of dynamic view to reveal the changes of China's energy usage and $\mathrm{CO}_{2}$ emissions, or failing use modellings to identify the main important factors for different dynamic indicators. It will be great worthy to enrich the current research via dynamically analysing the pattern of energy usage and its $\mathrm{CO}_{2}$ emission by the dynamic indicators, and statistic tools to identify the driving influencing factors. Therefore, this will be the goal of Chapter 7 . 


\section{Chapter 4}

\section{Re-configure the Existing Monitoring Stations Network}

\subsection{Framework}

The framework of reconfiguring will elaborate the general idea and the main steps for reconfiguring the existing monitoring network. Based on it, a research flow for the special case study was conducted focusing on technique parts. The criterion is considered as an important component of the whole reconfiguring process, which deserves a special description. It has to be mentioned that the findings of the result in this Chapter have been submitted to the journal Environment Modelling and Software.

\subsubsection{Framework for reconfiguring}

In order to provide useful tools for decision makers, several problem requirements need to be addressed

- To work with the full network or with a subset of it, depending on the interests or requirements of the decision makers.

- To accept a list of potential targeted places to locate the new or removed stations. 
- To define the pollutant relevant to the design of the network or, when several of them are significant, their relative weights.

When the problem has been stated, the first step is to produce a description and some statistical analysis from the existing monitoring network. This can help decision makers to recognize whether the selected network of stations is sparse or dense. The critical aspect of the methodology is to establish the relevant features and regressors, thereby making it possible to obtain suitable forecasting models. By using these forecasting models, the capability of using software sensors to predict the pollution values within the tolerance levels will be checked.

Models of highest quality need to be employed in order to obtain as much information as possible. Accurately predicting the pollution through modeling is of importance to ensure that accurate and sufficient information is obtained. Therefore, extra effort should be given to the modeling process, which is illustrated later by a case study in Figure 4-2.

Figure 4-1 illustrates the framework for reconfiguring an existing pollution network. To remove stations, the datasets are based on the target stations and its supported stations( that are to be removed or added). Various datasets are generated to obtain sufficient information from the prediction models. The best model is selected by using a statistical comparison of some methods that involve combinations of various feature selection methods and data mining methods training different prepared datasets by the modeling processes that are shown in Figure 4-2. These datasets (Dataset single-site and regional-site) not only are used for optimal models selection, but also for importance factor analysis of target pollutant and the sensitivity analysis because of sample size and temporal variations (from Dataset sampling and temporal). This is essential to assess the robustness of the predictive modeling.

By evaluating and comparing the models, the optimal method is obtained. The value of the information depends on various criteria according to the state of the art of target stations or supported stations. It can be gathered by use of the optimal method and other category datasets, namely Dataset Support in Figure 4-2. The Datasets Support is highly relevant to the network reconfiguration decision, which 
is obtained by varying inputs from different stations, but keeping the output from the same supported station. In such a case, the criteria can be either the mutual information and information gain that was calculated with the predicted values of the target pollutant, or measured by the prediction errors increasing/decreasing by the comparison of the Datasets Support.

To add a new station, the similar approach can be applied. Nevertheless, the mobile monitoring stations or the new smart sensors must be employed in order to select a short- term meteorological and pollution values at the candidate places. These new records are used to validate the quality of the software sensor, if Kriging or any other algorithm is used to interpolate the pollution level at the candidate places. In order to propose a prioritized list of places at which to establish the new stations, such a list can be ordered by the information criteria (e.g., information gain). Then, the datasets should be prepared from both the target station and the supported mobile monitoring stations at the candidate places (Step 6 in Figure 4-1).

The next step is to list the stations at which the models are better able to predict the measured pollution along with their support stations (the ones used to build the models) ranked by the selected criteria. The suggested stations in the dense network can be removed easily according to the selected criteria, such as Mean Square Error(MSE) in the following case study. Alternatively, a station that contributes the highest value of information can be filled to the place in a sparse network

The aforementioned modeling error is the weighted average of the modeling errors for the pollutants considered at the stations according to the established configuration. Therefore, this methodology can be used for one or several pollutants.

\subsubsection{Research flow for the Mexico City case}

In this section, a case study based on Mexico City is carried out to further explain how to reconfigure the existing pollution monitoring stations. The adopted strategy is to keep the focus on the algorithm side of the methodology, to emphasize the technical detail, which is crucial for its feasibility. As far as the detail is relevant, it was described neither paying attention to the qualification of the new places nor to 


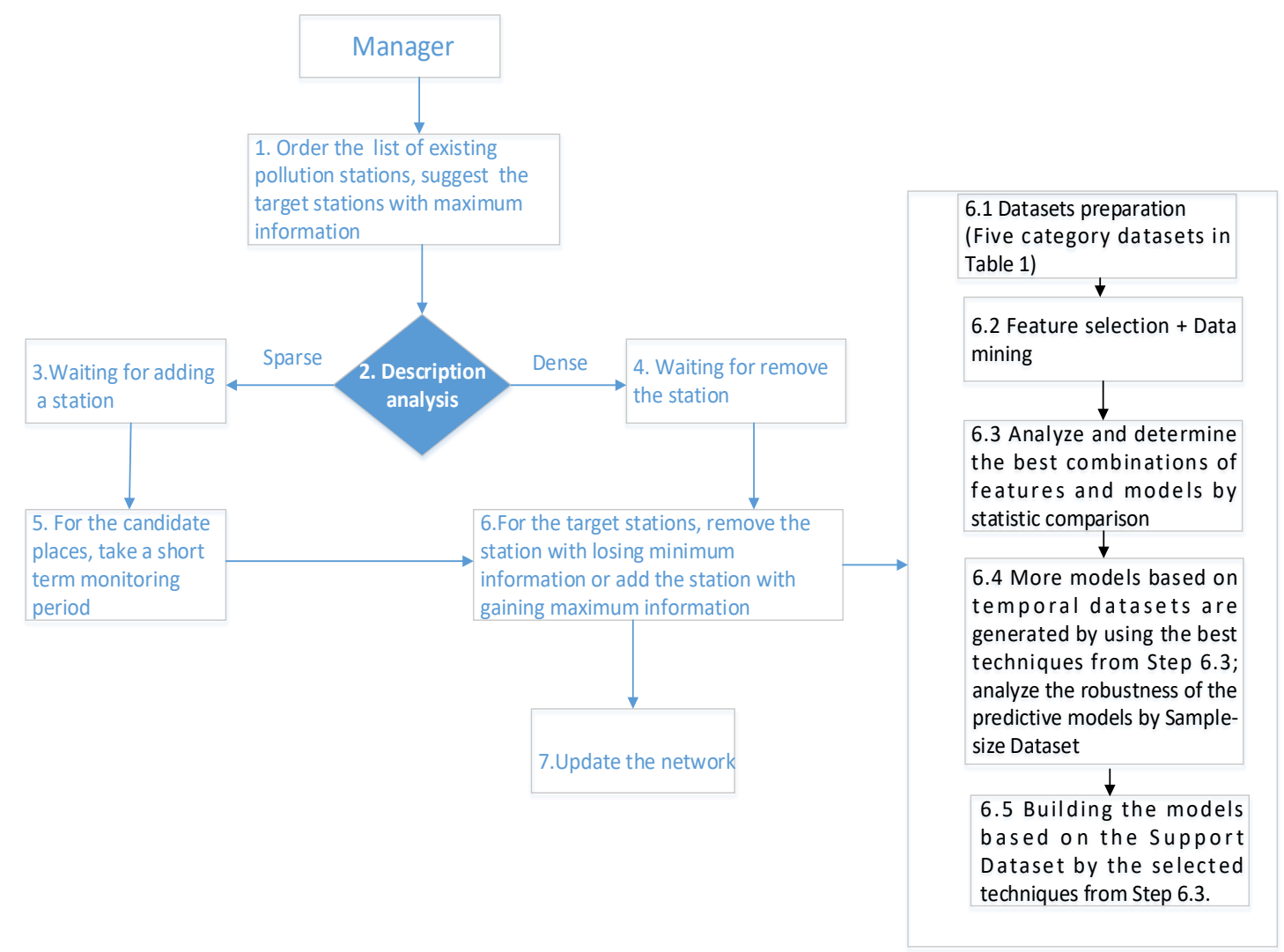

Figure 4-1: Framework for reconfiguring an existing pollution monitoring network.

the details of the interpolation techniques. Therefore, a small set of stations has been considered for reconfiguration and only one complex pollutant - ozone, was selected.

The station with highest ozone pollution level is given priority as the study target. Its nearby stations were applied for potential reduction of stations. Moreover, we have selected a set of clustered stations in a highly polluted city area, which has sufficient meteorological and pollution data can be obtained to guarantee enough samples for learning models. Finally, the target station PED, SUR, and TPN are selected and highlighted in Figure 2-2.

As the framework for reconfiguring was established, the forecasting model quality is the critical part of the whole methodology because those models are trained from the historical data looking to capture local dependence etc. Therefore, it does make sense to depict the details of the whole modeling process, starting from considering several features sets and learning sets. 
The details are presented graphically in Figure 4-2, where different logical steps expanded into different operations and along with the relationship with the proposed methodology steps in Figure 4-1, in order to emphasize the relationship between the case study and the methodology. In addition, the details of the datasets and methods used are described in Chapter 2, subsections 2.1.1, 2.2.1, 2.2.3 and 2.2.6.

\subsection{Description analysis}

Figures 4-3 - 4-6 illustrate the basic description of the characteristics of ozone formation in Mexico City. Mexico City is one of the most polluted cities in the world. It suffers a high-level ozone pollution problem. Although some programs or plans for improving the air quality have been implemented, the ozone levels in the metropolitan area of Mexico are still high. According to Figure 4-3, there are still a number of days in which that daily maximum ozone level exceeds the limitation (0.11 ppm) set up by the government, at air quality monitoring stations from 2009 to 2012. For stations PED and SUR, the number of days exceeding the threshold is higher than the numbers at other stations.

There is a strong correlation between ozone and other factors from local areas, described in Figure 4-4. For example, the positive correlation of $\mathrm{O}_{3}$ and temperature (TMP), negative correlation between $\mathrm{O}_{3}$ and $\mathrm{NO}_{x}$, relative humidity $(\mathrm{RH})$, and $\mathrm{CO}$ at the local PED station.

One of the main characteristics of ozone in Mexico City is that the variances of the ozone among the neighborhood monitoring stations are not significant, which is quite different from the ozone formation pattern in other cities, such as Beijing [59, 58]. The ozone monitoring stations in Mexico City are qualified as a dense network. Figure 4-5 and Figure 4-6 demonstrate the $\mathrm{O}_{3}$ level at PED, as well as its nearby and far away monitoring stations in the short-run period of time. There is no remarkable difference between these nearby stations in terms of hourly ozone records. Meanwhile, the ozone records follow one pattern, that is, peaking in the day time around 08:00 to 12:00 and falling to its lowest level at around 23:00 until 03:00. 


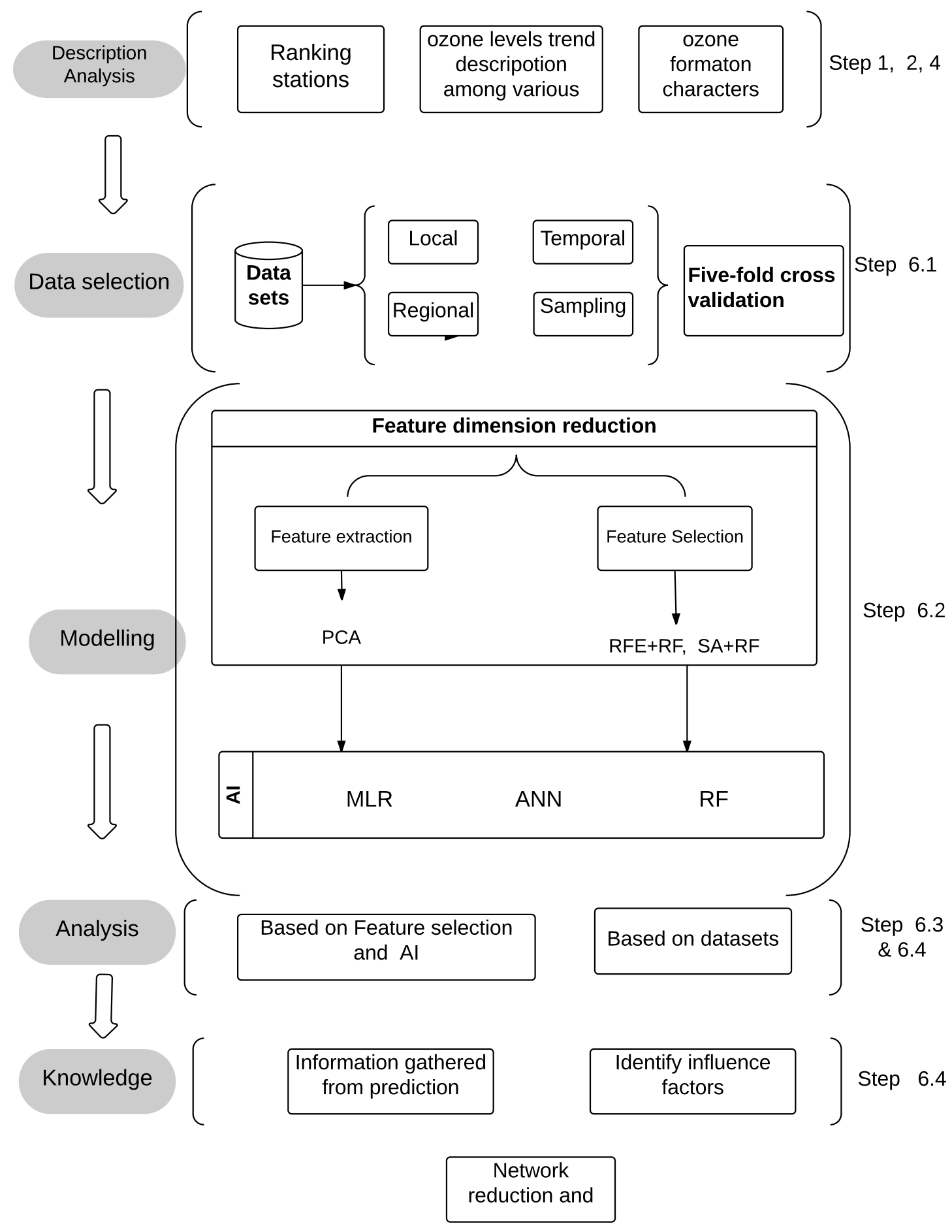

Figure 4-2: Research flow for the case study 
Consequently, the number of existing monitoring stations probably can be reduced given that their recorded ozone levels are quite similar and the ozone prediction at one station by using the data from this station can be performed as accurately as the prediction based on data from other stations. In such a case, managers are able to develop different strategies to identify the most convenient stations to be relocated.
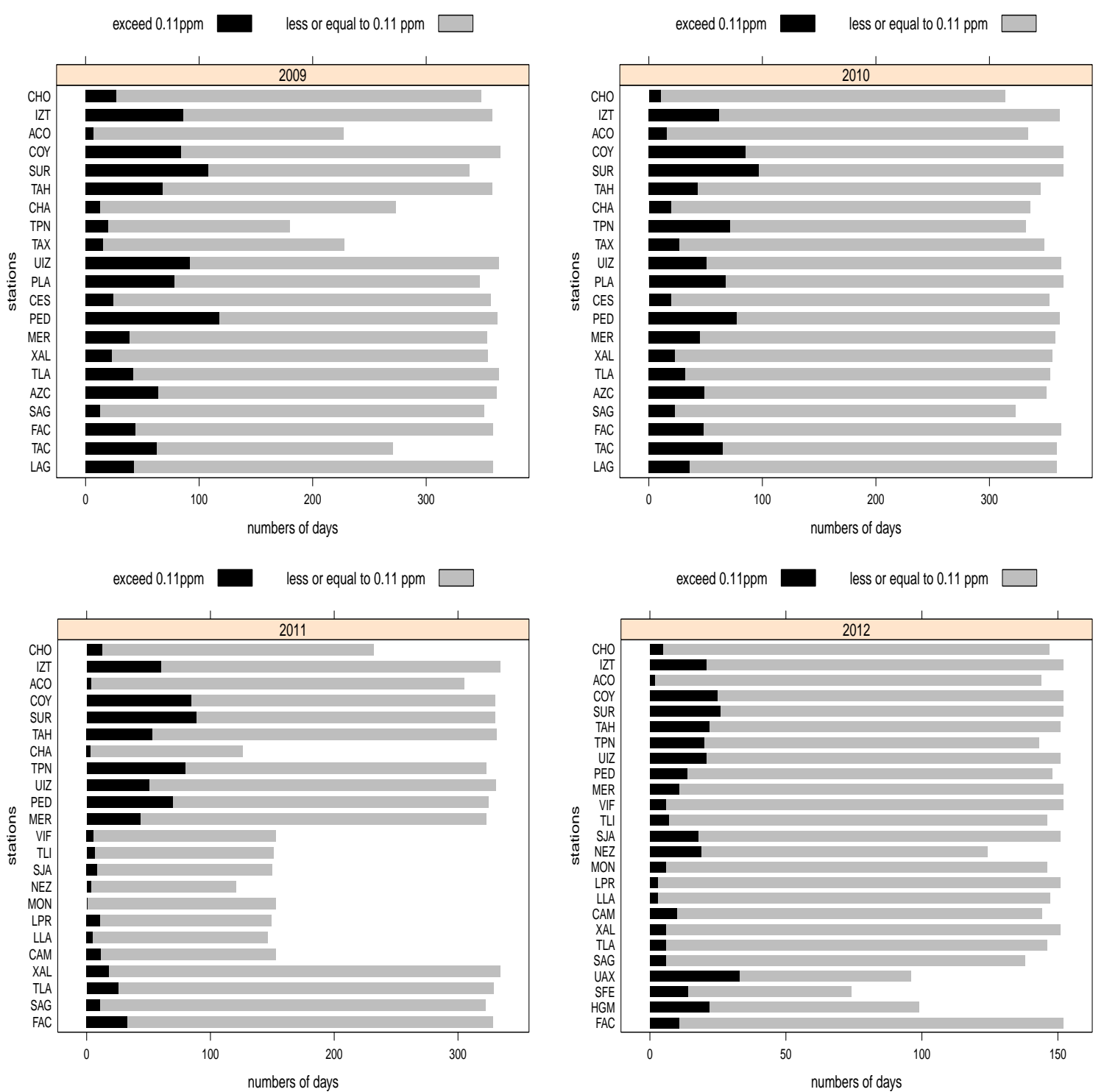

Figure 4-3: Numbers of days that the daily maximum ozone level exceeded the limitation (0.11 ppm) against numbers of days when it did not exceed or equal the limitation at all stations by year: 2009, 2010, 2011, 2012. 


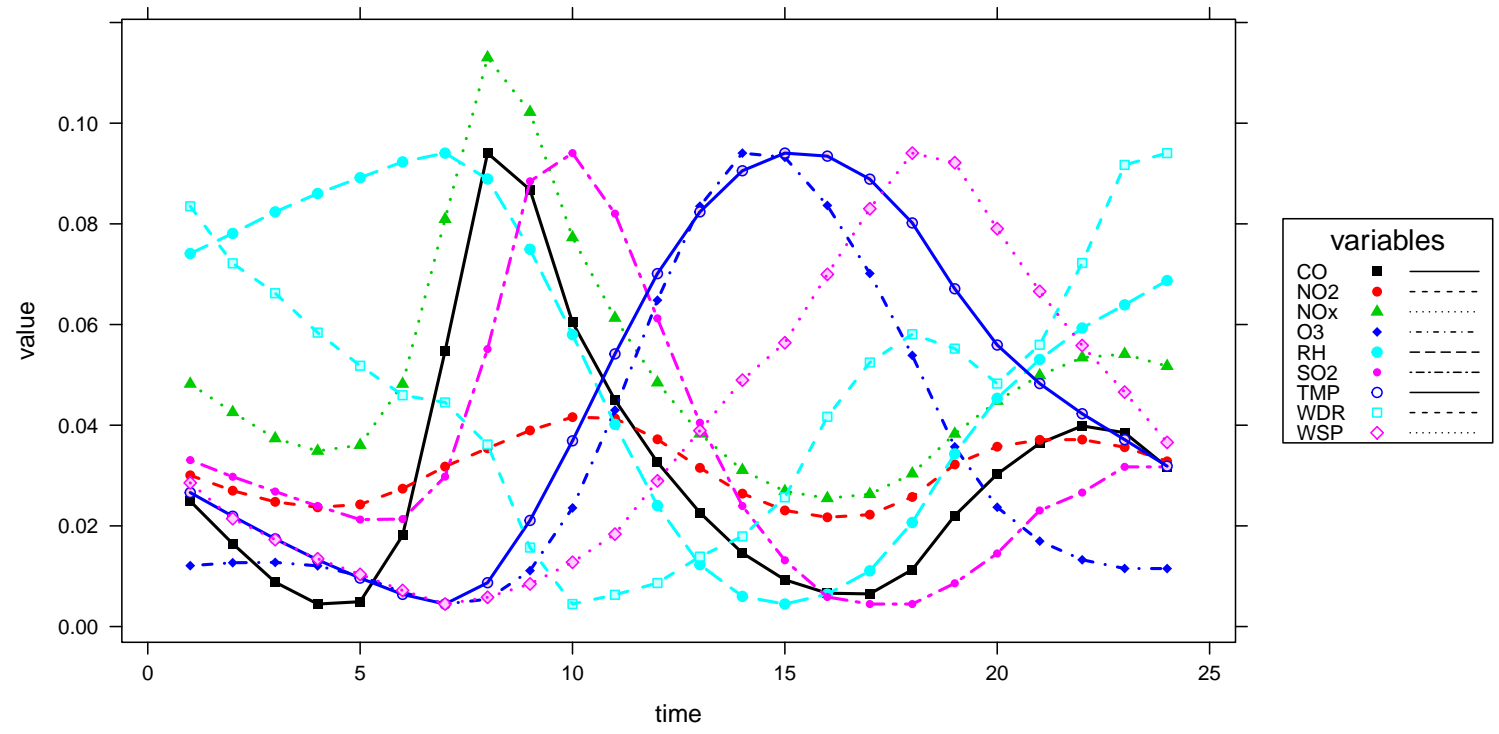

Figure 4-4: Hourly average value corresponding to different variables at station PED.

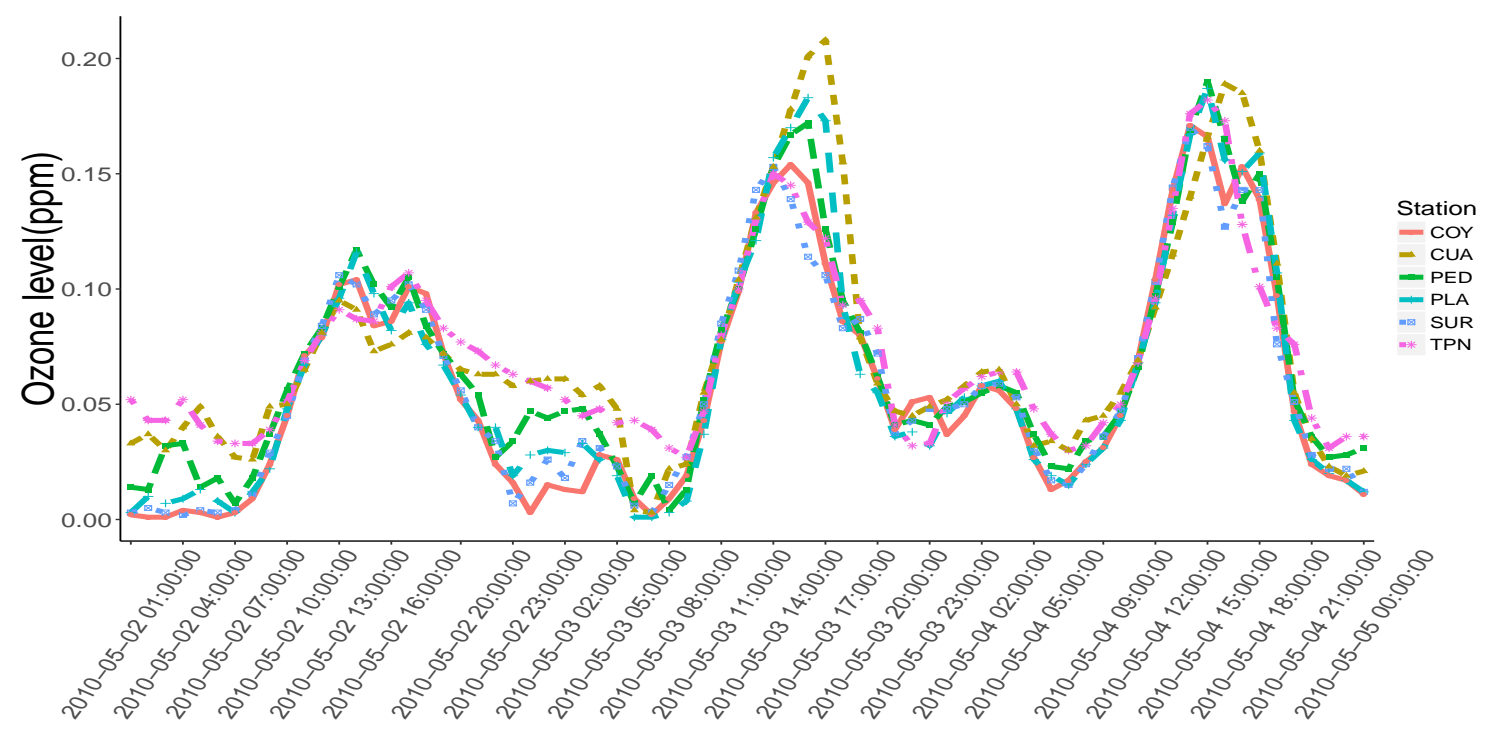

Figure 4-5: The hourly ozone levels of PED and its nearby stations at days 2010-05-02, 2010-05-03 and 2010-05-04. 


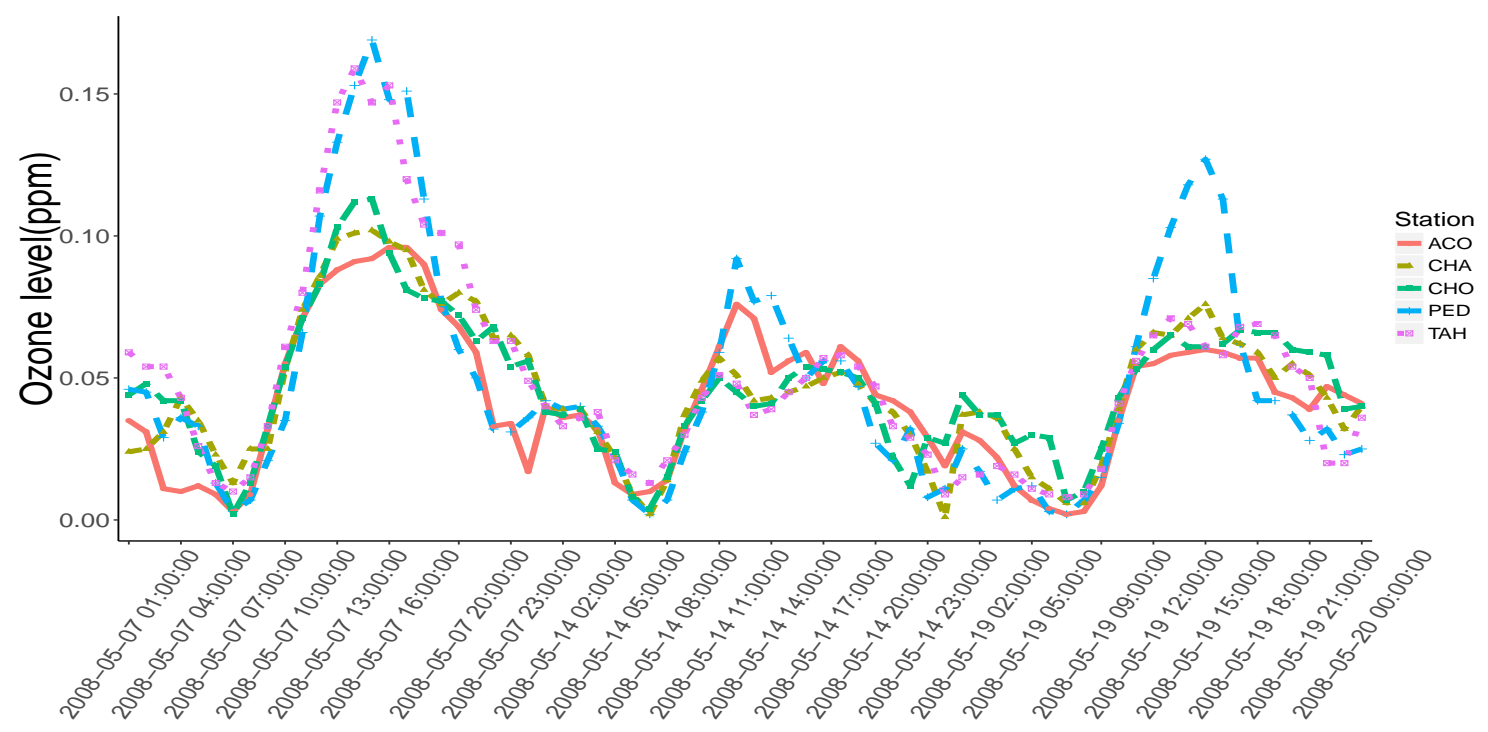

Figure 4-6: The hourly ozone levels of PED and its far stations at day 2008-05-07, 200805-14 and 2008-05-19.

\subsection{Feature extraction and selection}

Taking Dataset 3 as an example, Table 4.1 shows the Pearson correlation matrices of some of the input variables. It demonstrates that there is multicollinearity among the input variables. Figure 4-7 shows the cumulative proportion for each dataset, based on which the number of principal components of the inputs for each data set can be determined. The selected principal components should explain at least $75 \%$ of the variation. Thus, the number of the components are 5, 9, 13 and 12 for Datasets 1, 2, 3, and 4, respectively, as illustrated in Figure 4-7. The new components are used for the MLR, ANN, and RF models in the next step, training the models.

Figure 4-8 and Figure 4-9 demonstrate the model performance (RMSE) against iterations for SA and RFE with RF implementation on Dataset 3. The errors of the first time input selection are high. Then, RF performances improve significantly before the first 40 iterations and stay stable afterwards. Finally, the numbers of the selected inputs based on PCA, SA, and RFE can be obtained. No matter which techniques are used, simplified models can be implemented by a new combination of the input variables. 


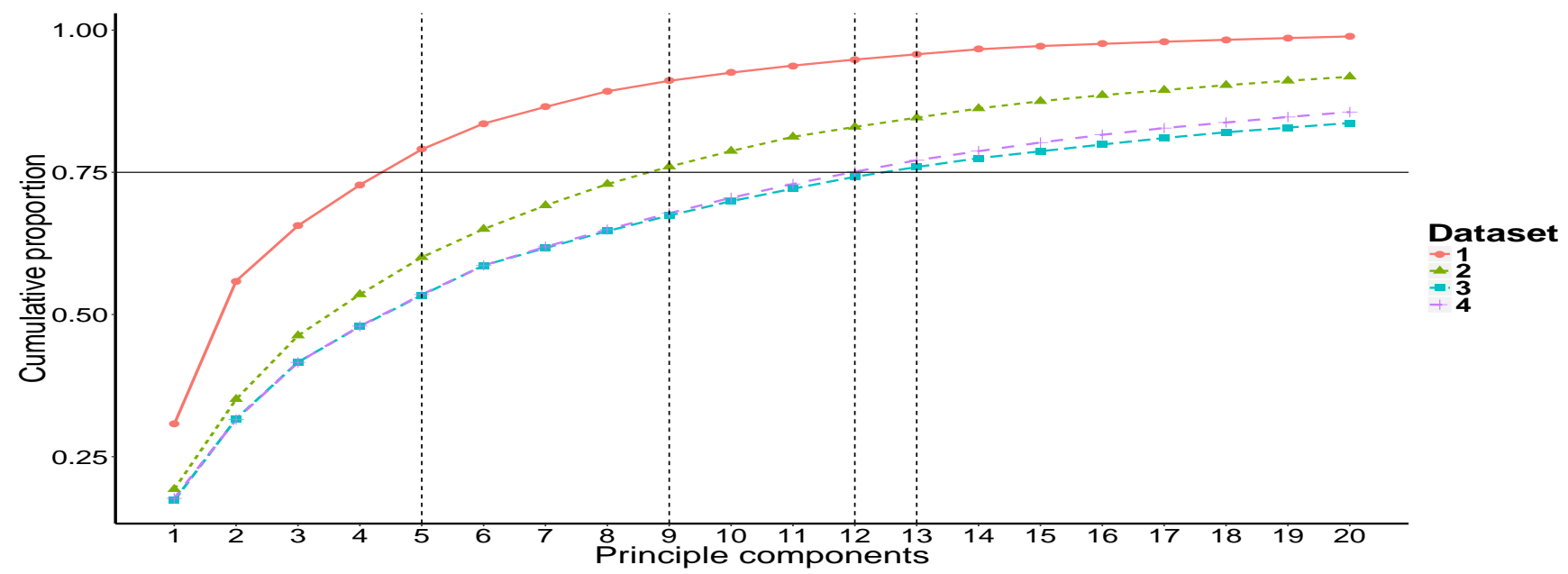

Figure 4-7: Cumulative proportion of PCA corresponding to Datasets 1 to 4.

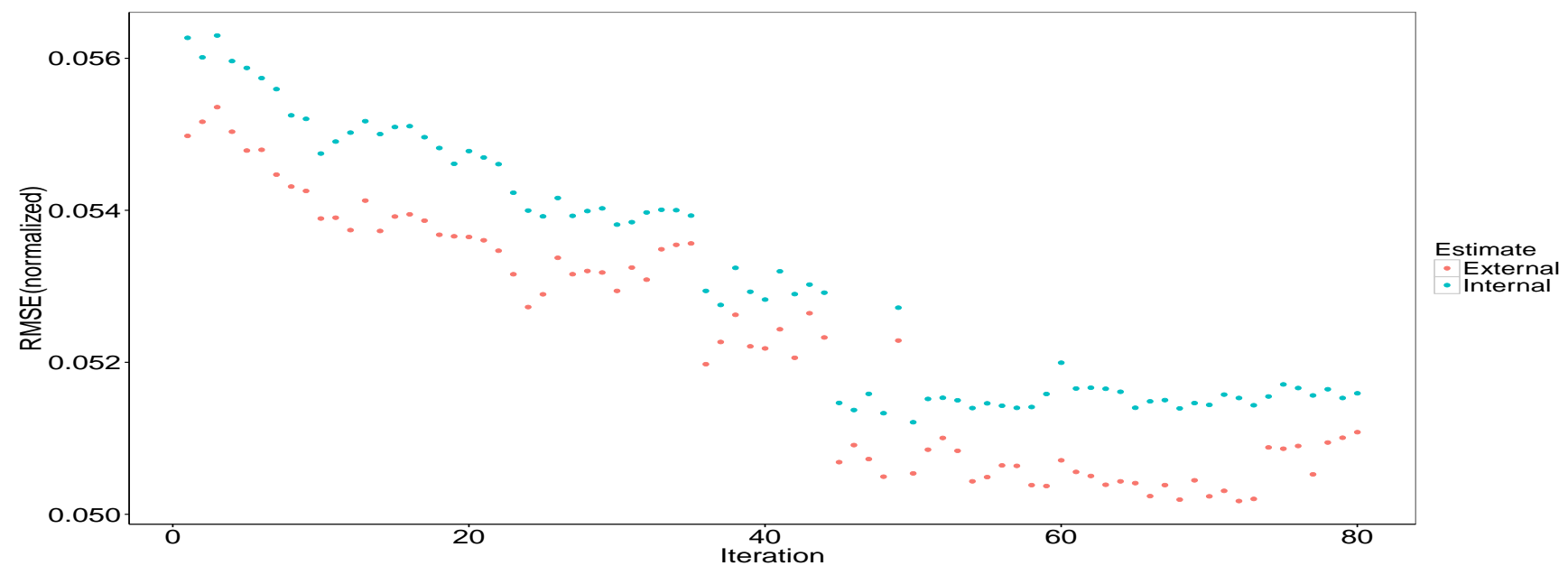

Figure 4-8: Errors of training data (internal) and validation dataset (external) (Dataset 3). 
Table 4.1: Pearson correlation matrix of the variables based on Dataset 3.

\begin{tabular}{lllllllll}
\hline & $\mathrm{M}$ & $\mathrm{D}$ & $\mathrm{H}$ & $\mathrm{S}$ & $\mathrm{W}$ & \multicolumn{2}{c}{$\max \left(\mathrm{O}_{3}\right)$} & \multicolumn{2}{c}{$\max \left(\mathrm{NO}_{x}\right) \max \left(\mathrm{NO}_{2}\right)$} \\
$\mathrm{M}$ & 1.000 & -0.033 & 0.036 & $\mathbf{0 . 9 8 3}$ & -0.077 & -0.013 & -0.008 & -0.030 \\
$\mathrm{D}$ & - & 1.000 & 0.010 & -0.027 & 0.026 & -0.025 & 0.081 & 0.091 \\
$\mathrm{H}$ & - & - & 1.000 & 0.032 & -0.005 & 0.369 & -0.060 & 0.152 \\
$\mathrm{~S}$ & - & - & - & 1.000 & -0.071 & -0.032 & 0.009 & -0.022 \\
$\mathrm{~W}$ & - & - & - & - & 1.000 & 0.068 & -0.102 & -0.011 \\
$\max \left(\mathrm{O}_{3}\right)$ & - & - & - & - & - & 1.000 & -0.224 & 0.007 \\
$\max \left(\mathrm{NO}_{x}\right)$ & - & - & - & - & - & - & 1.000 & $\mathbf{0 . 7 2 0}$ \\
$\max \left(\mathrm{NO}_{2}\right)$ & - & - & - & - & - & - & - & 1.000 \\
\hline
\end{tabular}

\subsection{Model performance}

Table 4.2, Table 4.3, and Table 4.4 demonstrate the model performances corresponding to the three evaluation matrices in Chapter 2, Subsection 2.2.6, compiled with the datasets used and models built. To investigate statistically significant differences among the types of artificial intelligence models and datasets based on which the models are built, a Wilcox Signed Rank test was conducted and the results are shown in Table 4.5 and Table 4.6 .

It should be noted that the RF models have the highest value of explained variances and lowest error compared to MLR and ANN for all datasets. Regarding the feature reduction method PCA, there is no significant decrease in forecasting accuracy for the MLR and ANN models. However, for RF models, it decreases the dimensions of the inputs at the expense of deteriorating the forecasting accuracy. PCA with RF models have around $41 \%-57 \%$ for MAE, $50 \%-86 \%$ for RMSE and $42 \%-78 \%$ for RELE higher than RF.

SA_ MLR and SA_RF, on the other hand, have a slight improvement compared to MLR and RF, respectively, based on the performance measurements MAE and RMSE. Moreover, according to the performance measurement RELE, RFE_ RF performs better than RF. 


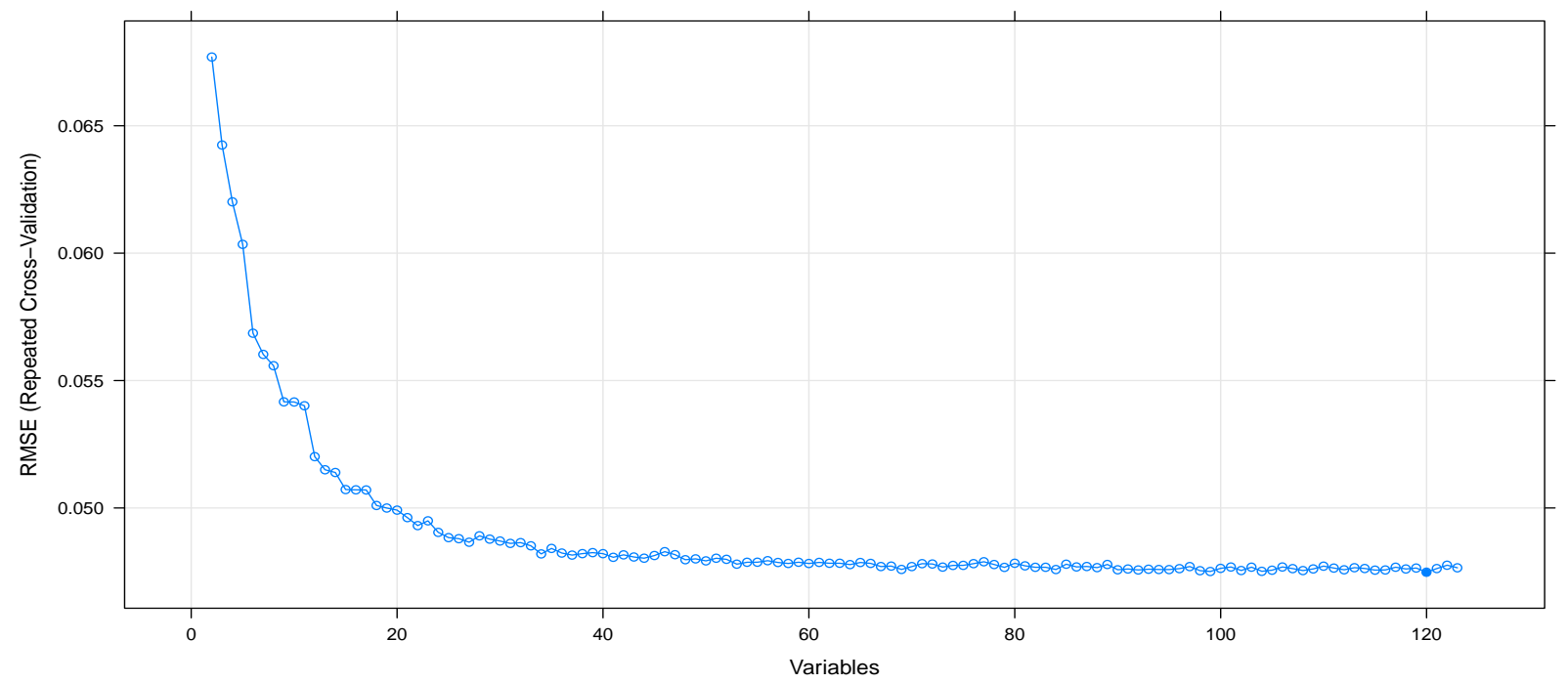

Figure 4-9: Errors (RMSE) of numbers of variables corresponding to RFE _ RF (Dataset 3).

Table 4.2: Model performance MAE of Datasets 1 to 4 .

\begin{tabular}{llllll}
\hline \hline FS & Models & 1 & 2 & 3 & 4 \\
\hline \hline - & MLR & 0.0146 & 0.0137 & 0.0558 & 0.0150 \\
PCA & MLR & 0.0155 & 0.0152 & $\mathbf{0 . 0 1 3 4}$ & 0.0148 \\
SA & MLR & 0.0144 & $\mathbf{0 . 0 1 3 5}$ & 0.0169 & $\mathbf{0 . 0 1 4 7}$ \\
RFE & MLR & $\mathbf{0 . 0 1 3 8}$ & 0.0138 & 0.0157 & 0.0154 \\
\hline - & ANN & 0.0147 & 0.0190 & 0.0219 & $\mathbf{0 . 0 1 2 6}$ \\
PCA & ANN & 0.0148 & $\mathbf{0 . 0 1 4 5}$ & 0.0151 & 0.0190 \\
SAF & ANN & 0.0167 & 0.0174 & $\mathbf{0 . 0 1 2 3}$ & 0.0167 \\
RFE & ANN & $\mathbf{0 . 0 1 3 3}$ & 0.0227 & 0.0154 & 0.0214 \\
\hline - & RF & 0.0103 & 0.0096 & $\mathbf{0 . 0 1 0 0}$ & 0.0101 \\
PCA & RF & 0.0148 & 0.0145 & 0.0147 & 0.0160 \\
SA & RF & $\mathbf{0 . 0 0 9 5}$ & $\mathbf{0 . 0 0 9 5}$ & 0.0106 & $\mathbf{0 . 0 0 9 6}$ \\
RFE & RF & 0.0103 & 0.0099 & 0.0100 & 0.0097 \\
\hline \hline
\end{tabular}


Table 4.3: Model performance RMSE of Datasets 1 to 4.

\begin{tabular}{llllll}
\hline \hline FS & Models & 1 & 2 & 3 & 4 \\
\hline \hline PCA & MLR & 0.0184 & 0.0174 & 0.0734 & 0.0186 \\
SA & MLR & 0.0183 & 0.0173 & 0.0202 & 0.0182 \\
RFE & MLR & 0.0175 & 0.0173 & 0.0202 & 0.0192 \\
\hline - & ANN & 0.0201 & 0.0256 & 0.0280 & 0.0160 \\
PCA & ANN & 0.0192 & 0.0186 & 0.0192 & 0.0210 \\
SA & ANN & 0.0226 & 0.0224 & 0.0162 & 0.0214 \\
RFE & ANN & 0.0177 & 0.0284 & 0.0218 & 0.0274 \\
\hline - & RF & 0.0134 & $\mathbf{0 . 0 1 2 3}$ & $\mathbf{0 . 0 1 3 0}$ & 0.0131 \\
PCA & RF & 0.0201 & 0.0191 & 0.0197 & 0.0245 \\
SA & RF & $\mathbf{0 . 0 1 2 3}$ & $\mathbf{0 . 0 1 2 3}$ & 0.0139 & $\mathbf{0 . 0 1 2 4}$ \\
RFE & RF & 0.0133 & 0.0129 & 0.0130 & 0.0127 \\
\hline \hline
\end{tabular}

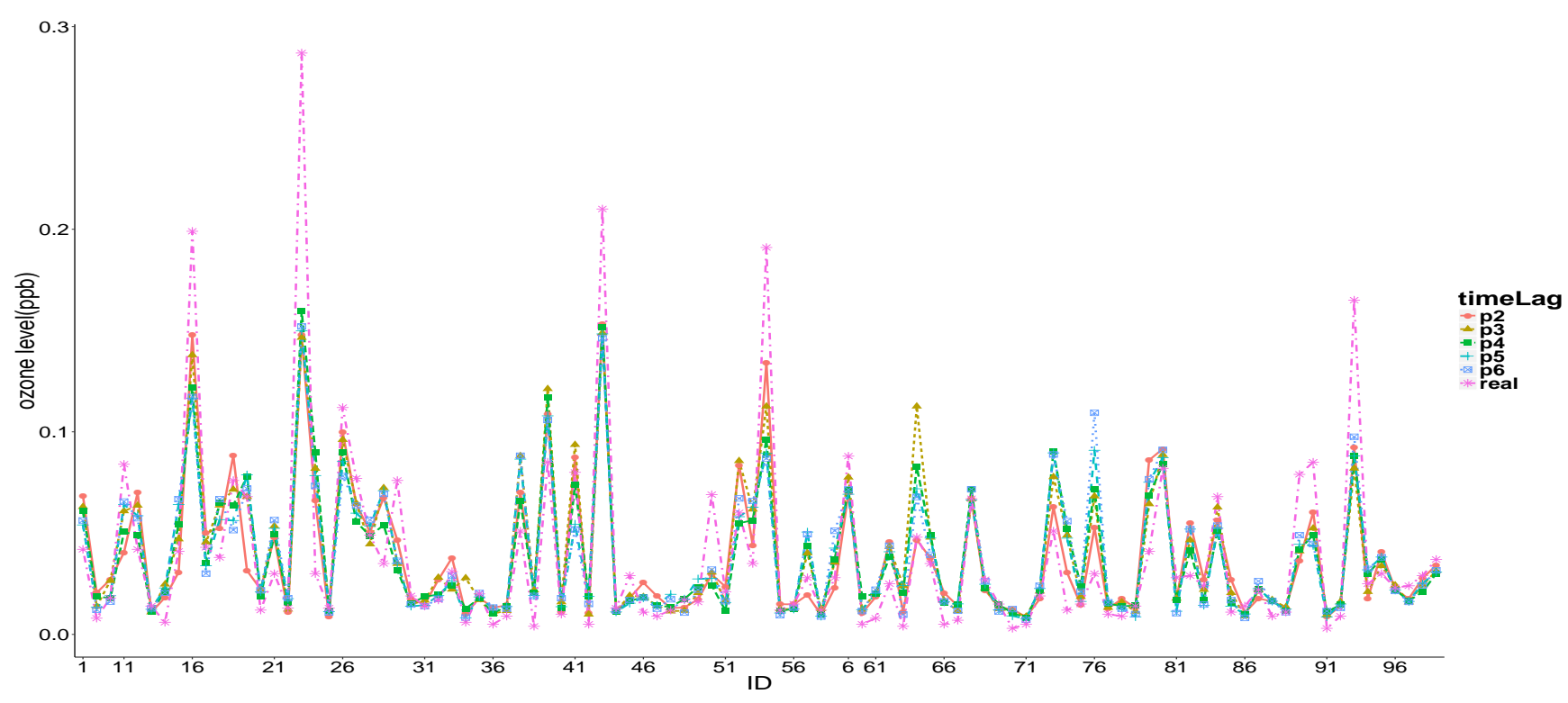

Figure 4-10: Observed and predicted values of RF model for the temporal datasets 
Table 4.4: Model performance RELE of Datasets 1 to 4.

\begin{tabular}{llllll}
\hline \hline FS & Models & 1 & 2 & 3 & 4 \\
\hline \hline PCA & MLR & 0.6420 & 0.6775 & 0.6147 & 0.6588 \\
SA & MLR & 0.5469 & 0.5897 & 0.7250 & 0.6360 \\
RFE & MLR & 0.5845 & 0.5837 & 0.6559 & 0.6783 \\
\hline- & ANN & 0.5931 & 0.6677 & 0.6781 & 0.5970 \\
PCA & ANN & 0.5228 & 0.5860 & 0.4672 & 0.6682 \\
SA & ANN & 0.6944 & 0.7471 & 0.4159 & 0.6089 \\
RFE & ANN & 0.5007 & 0.8417 & 0.6652 & 0.6948 \\
\hline- & RF & 0.3539 & 0.3835 & 0.3762 & 0.3731 \\
PCA & RF & 0.5354 & 0.6823 & 0.5488 & 0.5975 \\
SA & RF & 0.3752 & 0.3752 & 0.3831 & 0.3639 \\
RFE & RF & $\mathbf{0 . 3 5 3 1}$ & $\mathbf{0 . 3 7 1 9}$ & $\mathbf{0 . 3 6 2 6}$ & $\mathbf{0 . 3 5 2 7}$ \\
\hline \hline
\end{tabular}

Furthermore, the RF model performances based on temporal datasets and sample sized data sets are presented in Table 4.7. The results indicate that the best prediction is obtained for predicting 2 hours ahead. The accuracy of the model goes down when the prediction time gets longer. The error increases around 15\% for MAE, $19 \%$ for RMSE, and $6 \%$ for RELE from predicting 2 hours ahead to 6 hours ahead. To check the forecasting capability, Figure 4-10 illustrates 100 samples of forecasting the ozone value of RF models and observed ozone values from test subsets. These results show that the RF models can achieve promising results. Even though it is hard for the RF models to achieve an accurate forecasting estimation for extreme ozone values, they can successfully be used for judging if the ozone exceeds the threshold (0.110 ppb), since the predicted values exceed $0.110 \mathrm{ppb}$ as well.

In addition, $\mathrm{RF}$ models based on sample size datasets demonstrate that the forecasting performances can be enhanced significantly by enlarging the samples. The 

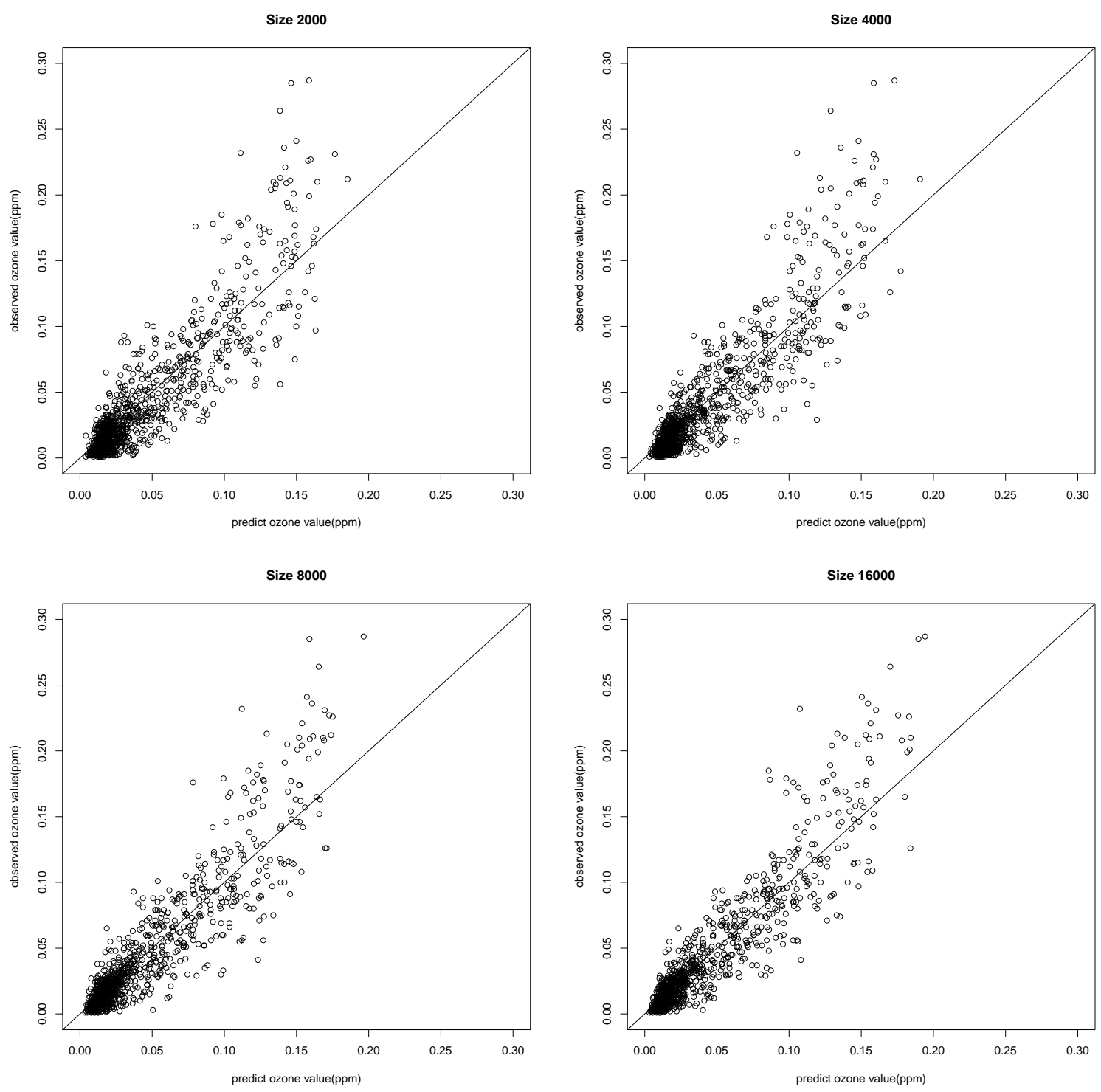

Figure 4-11: Predicted and observed values based on sampling size datasets 
Table 4.5: Results of Wilcox signed rank test to investigate statistically significant differences among the datasets

\begin{tabular}{lcccccc}
\hline \hline & \multicolumn{2}{c}{ MAE } & \multicolumn{2}{c}{ RMSE } & \multicolumn{2}{c}{ RELE } \\
\hline Dataset & Mean_ & P-value & Mean_ & P-value & Mean_ & P-value \\
& diff & & diff & & diff & \\
\hline 1 VS 2 & -0.0009 & 0.9081 & 0.0397 & 0.4188 & -0.0719 & 0.1005 \\
1 VS 3 & 0.1063 & 0.0242 & -0.0615 & 0.7987 & -0.2047 & 0.3186 \\
1 VS 4 & -0.0011 & 0.4096 & -0.0397 & 0.7987 & -0.0634 & 0.0684 \\
2 VS 3 & -0.1054 & 0.0734 & -0.1012 & 0.9539 & -0.1328 & 0.7125 \\
2 VS 4 & 0.0002 & 0.5512 & -0.0004 & 0.7987 & 0.0085 & 0.8428 \\
3 VS 4 & 0.1052 & 0.1560 & 0.1012 & 0.9774 & 0.1413 & 0.8874 \\
\hline$* p$-value significant at $\alpha=0.05$ & & & & \\
$* * p$-value significant at $\alpha=0.01$ & & & &
\end{tabular}

error MAE decreased by around $19 \%$ from 2000 to 16,000 training samples. the RMSE goes down by around 14\%, and RELE by $32 \%$.

\subsection{Feature importance}

This study conducted importance analyses of the variables in order to understand the characteristics of the ozone formation at the PED station in Mexico City. Table 4.8 illustrates the top ten important variables based on the model RF corresponding to Datasets 2, 3 and 4 .

The variable scores of WDR, $\mathrm{O}_{3}$, TMP and $\mathrm{NO}_{x} / \mathrm{NO}_{2}$ of the current day at the PED station based on RF models are higher than the other variables for Dataset 2. The variables from the previous day are all out of the top 15, which means these variables have less to contribute to a forecasting improvement than the variables of the current day. Moreover, according to the ranks of the variables on Datasets 3 and 4, the WDR variables still dominate, with the top importance. The scores of the 
Table 4.6: Results of Wilcox signed rank test based on MAE

\begin{tabular}{|c|c|c|c|c|c|c|c|c|c|c|c|c|c|}
\hline & & - & PCA & $\mathrm{SA}$ & RFE & - & PCA & SA & RFE & - & PCA & SA & RFE \\
\hline FS & Mode & elsMLR & MLR & MLR & MLR & ANN & ANN & ANN & ANN & $\mathrm{RF}$ & $\mathrm{RF}$ & $\mathrm{RF}$ & $\mathrm{RF}$ \\
\hline - & MLR & - & 1.0000 & 0.6857 & 1.0000 & 1.0000 & 0.8857 & 0.8857 & 0.8857 & 0.0286 & $* 1.0000$ & 0.028 & $6^{*} 0.0286^{*}$ \\
\hline PCA & MLR & - & - & 0.8857 & 0.8857 & 0.8857 & 1.0000 & 0.3429 & 0.4857 & 0.0286 & $* 1.0000$ & 0.028 & 60.0286 \\
\hline SA & MLR & - & - & - & 1.0000 & 0.6857 & 0.3429 & 0.6857 & 0.4857 & 0.0286 & ;*. 6857 & 0.028 & $6^{*} 0.0286^{*}$ \\
\hline RFE & MLR & - & - & - & - & 0.6857 & 0.6857 & 0.3429 & 0.4857 & 0.0286 & $* 0.6857$ & 0.0286 & 6*0.0286 \\
\hline - & ANN & - & - & - & - & - & 0.8857 & 0.6857 & 0.6857 & 0.0286 & $* 0.8857$ & 0.028 & $6 * 0.0286^{*}$ \\
\hline PCA & ANN & - & - & - & - & - & - & 0.8857 & 0.4857 & 0.0286 & ;*0.8857 & 0.028 & $6^{*} 0.0286^{*}$ \\
\hline SA & ANN & - & - & - & - & - & - & - & 0.6857 & 0.0286 & ;*0.3429 & 0.028 & $6^{*} 0.0286^{*}$ \\
\hline RFE & ANN & - & - & - & - & - & - & - & - & 0.0286 & * *. 4857 & 0.028 & $6 * 0.0286^{*}$ \\
\hline- & $\mathrm{RF}$ & - & - & - & - & - & - & - & - & - & 0.0286 & ;*0.6857 & 1.0000 \\
\hline PCA & $\mathrm{RF}$ & - & - & - & - & - & - & - & - & - & - & 0.028 & $6^{*} 0.0286^{*}$ \\
\hline SA & RF & - & - & - & - & - & - & - & - & - & - & - & 0.6857 \\
\hline
\end{tabular}

$* p$-value significant at $\alpha=0.05$

** $p$-value significant at $\alpha=0.01$

Table 4.7: RF model performance for time ahead prediction and various sizes of training data

\begin{tabular}{llllll|llll}
\hline & $2-\mathrm{p} 2$ & $2-\mathrm{p} 3$ & $2-\mathrm{p} 4$ & $2-\mathrm{p} 5$ & $2-\mathrm{p} 6$ & 2-s2000 & 2-s4000 & 2-s8000 & 2-s16000 \\
\hline MAE & 0.01496 & 0.01616 & 0.01667 & 0.016733 & 0.017047 & 0.01496 & 0.01401 & 0.01306 & 0.01220 \\
RMSE & 0.02261 & 0.026279 & 0.027022 & 0.027418 & 0.027658 & 0.02261 & 0.02231 & 0.02072 & 0.01953 \\
RELE & 0.90945 & 0.836572 & 0.881224 & 0.875163 & 0.883209 & 0.90945 & 0.76318 & 0.69077 & 0.61742 \\
\hline
\end{tabular}



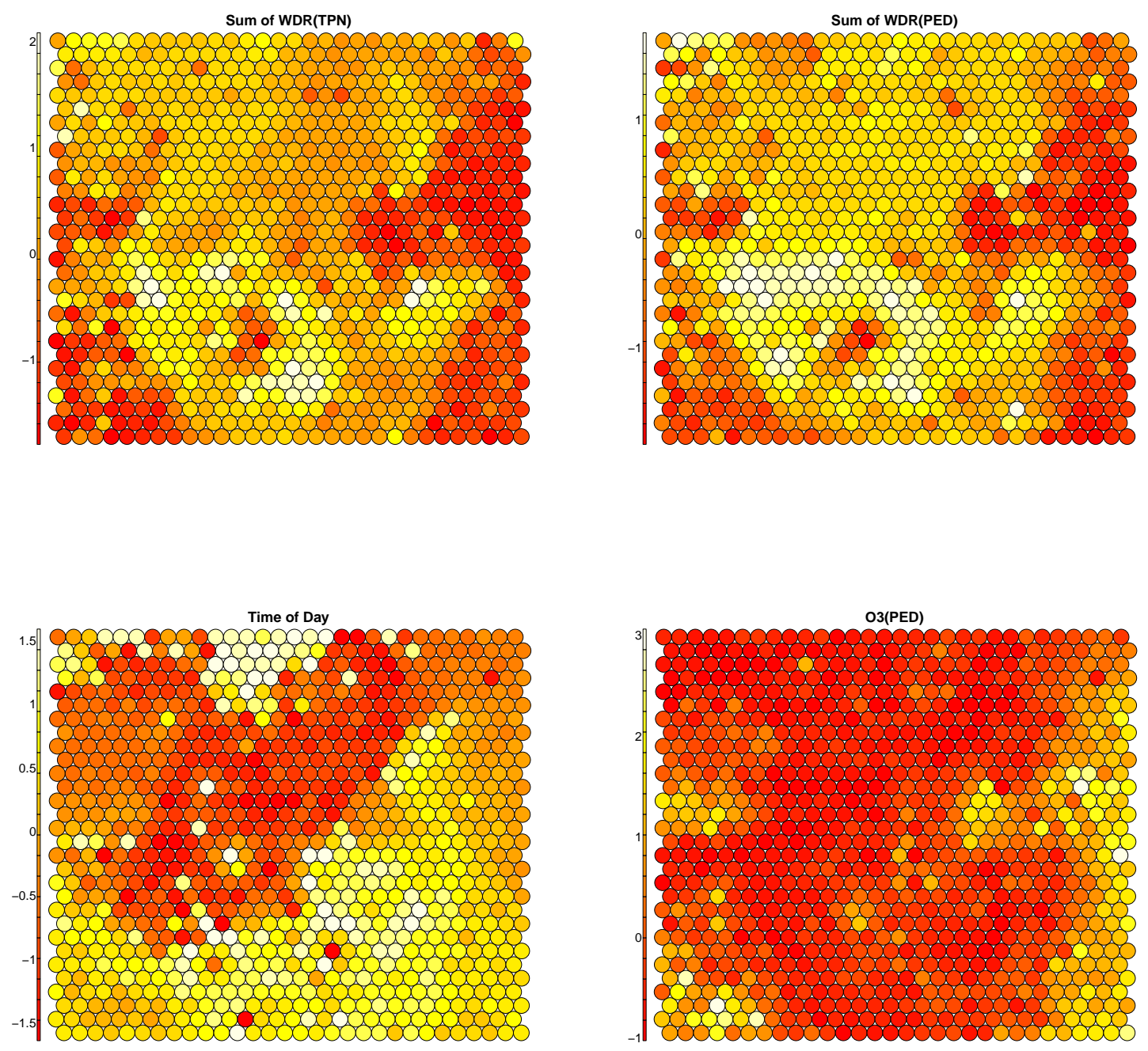

Figure 4-12: Heatmaps of Self-Organized Mapping according to variables. 
Table 4.8: The rank of the first fifteen important variables by RF corresponding to Datasets 2,3 and 4

\begin{tabular}{lll|ll|ll}
\hline \hline Rank & PED & score & SUR $+\mathrm{TPN}$ & score & PED+SUR+TPN & score \\
\hline 1 & median(WDR $)_{P E D}$ & 6.647 & $\operatorname{sum}(\mathrm{WDR})_{T P N}$ & 4.1128 & $\mathrm{sum}(\mathrm{WDR})_{T P N}$ & 2.9209 \\
2 & $\mathrm{H}$ & 4.4556 & $\operatorname{median}(\mathrm{WDR})_{T P N}$ & 3.7407 & $\operatorname{median}(\mathrm{WDR})_{T P N}$ & 2.8753 \\
3 & $\operatorname{mean}(\mathrm{WDR})_{P E D}$ & 2.9266 & $\operatorname{mean}(\mathrm{WDR})_{T P N}$ & 2.6158 & $\max (\mathrm{WDR})_{T P N}$ & 2.4736 \\
4 & $\mathrm{sum}(\mathrm{WDR})_{P E D}$ & 2.3353 & $\max (\mathrm{WDR})_{T P N}$ & 2.6037 & $\operatorname{mean}(\mathrm{WDR})_{T P N}$ & 2.398 \\
5 & $\min (\mathrm{WDR})_{P E D}$ & 1.6908 & $\min (\mathrm{WDR})_{T P N}$ & 2.495 & $\min (\mathrm{WDR})_{T P N}$ & 1.9101 \\
6 & $\max \left(\mathrm{O}_{3}\right)_{P E D}$ & 1.2479 & $\mathrm{H}$ & 2.2097 & $\mathrm{H}$ & 1.5028 \\
7 & $\max (\mathrm{TMP})_{P E D}$ & 1.2305 & $\max (\mathrm{O})_{S U R}$ & 0.9402 & $\operatorname{sum}(\mathrm{WDR})_{P E D}$ & 1.0526 \\
8 & $\operatorname{Max}\left(\mathrm{WDR}_{P E D}\right.$ & 1.0492 & $\max (\mathrm{TMP})_{T P N}$ & 0.6228 & $\operatorname{median}(\mathrm{WDR})_{P E D}$ & 0.9888 \\
9 & $\operatorname{mean}\left(\mathrm{O}_{3}\right)_{P E D}$ & 0.8364 & $\max (\mathrm{WSP})_{S U R}$ & 0.5913 & $\operatorname{mean}(\mathrm{WDR})_{P E D}$ & 0.8595 \\
10 & $\min \left(\mathrm{NO}_{x} / \mathrm{NO}_{2}\right)_{P E D}$ & 0.8252 & $\operatorname{sum}(\mathrm{TMP})_{T P N}$ & 0.557 & $\min (\mathrm{WDR})_{P E D}$ & 0.7921 \\
\hline \hline
\end{tabular}

variable WDR at TPN is higher than the one at PED and SUR. In addition, the variable $\mathrm{H}$ plays a vital role in $\mathrm{RF}$ models corresponding to Datasets 2,3 and 4 . This is mainly because the time of the day $(\mathrm{H})$ normally corresponds to solar radiation, which is an important factor for the formation of $\mathrm{O}_{3}$.

Moreover, this study constructed Self-Organising Maps for the variable sum(WDR $)_{T P N}$, sum(WDR $)_{P E D}$, variable $\mathrm{H}$, and $\mathrm{O}_{3}$ at station PED to present a visualization of the distribution of each variable across the map (Figure 4-12). It is noteworthy that the heatmaps show an inverse correlation among sum(WDR $)_{T P N}$ and $\mathrm{O}_{3}, \operatorname{sum}(\mathrm{WDR})_{P E D}$ and $\mathrm{O}_{3}$.

\subsection{Removing the stations with losing a minimum information}

Table 4.9 analyzes the target stations (SUR and TPN) and their support stations from which the input data was extracted. For the reduction of the existing monitoring 
Table 4.9: Results of reducing the number of stations.

\begin{tabular}{lllllll}
\hline Dataset & Input & Output & Target(remove) & MAE & RMSE & RELE \\
\hline 5 & PED & SUR & SUR & 0.0221 & 0.0292 & 2.1184 \\
6 & TPN & SUR & SUR & 0.0105 & 0.0143 & 0.2479 \\
7 & SUR & SUR & SUR & 0.0113 & 0.0149 & 0.8118 \\
\hline 8 & PED & TPN & TPN & 0.0189 & 0.0255 & 0.6337 \\
9 & SUR & TPN & TPN & 0.0110 & 0.0151 & 0.3297 \\
10 & TPN & TPN & TPN & 0.0095 & 0.0131 & 0.2467 \\
\hline
\end{tabular}

network, it is important to first determine how much information will be lost if the supported station are removed. The errors of the predictive models by using Datasets 6 to 10 can be used for this purpose. This will be explained later.

Taking MAE as the measurement criterion, the error MAE based on Dataset 6 is 7.1\% lower than the model based on Dataset 7. This means that, if SUR is removed, the ozone at SUR can be predicted more accurately by using the data from TPN than from SUR and PED. In another words, there is no information loss by removing the SUR station.

For the model based on Dataset 9, the performance deteriorates by around $15 \%$ (MAE) and 89.9\% compared to Dataset 10 and Dataset 8, respectively. This means that the ozone prediction accuracy decreases by $15 \%$ when using the data from SUR only, rather than from TPN and PED. In that case, if the TPN station is removed, the loss of information at TPN station measured by MAE will be at least $15 \%$.

An acceptable level of information loss depends on the requirements of the decision makers. For instance, if the information loss threshold (measured by MAE) is less than $10 \%$, it is recommended that station SUR be removed, but TPN kept in this case. Later, the existing ozone monitoring station network can be updated. The stations that have the highest importance, and their nearby stations, are selected as the new target ones for the next network reconfiguration. 


\subsection{Results and discussion}

The main findings of our study can be summarized as following:

1 A new methodology was proposed and it was applied on a case study of reconfiguration of existing pollution monitoring network.

2 Based on the case study, the state-of-the-art machine learning - RF can improve model performance significantly compared to the standard application of MRL and ANN.

$3 \mathrm{SA}$ and RFE feature selection implemented with RF models can reduce the numbers and select the optimal combination of input features, and build simpler models can without losing forecasting accuracy. PCA, one the other hand, deteriorates RF forecasting accuracy considerably by reducing the inputs dimensions and simplify the training model.

4 From the input importance analysis, the meteorological factors, especially wind direction, wind speed and ozone levels from TPN station, are the key factor to the attribution of ozone formation at PED station area. Wind Direction has adverse relation with $O_{3}$ by SOMs analysis.

5 Since increasing the size of samples, models performance can be improved, which contribute to the robustness of the system.

6 Through the feature selection and AI models' comparison, the maximum information can be gathered by using the best prediction FS an AI model. Then, monitoring and accurate pollution prediction for one monitoring station is able to be generated by the information from other stations, making it possible to move or remove the target station and update the whole network. 


\section{Chapter 5}

\section{Forecast Ozone Levels: Dealing with Unbalanced Datasets}

In this chapter, two main results will be obtained. One is the finding of identifying potential influences of social economic activities factors of various pollutants; The other one is the applications of the artificial intelligence techniques on ozone forecasting. Both are based on Hong Kong areas. It has to be mentioned that the findings regarding the second part have been published in the journal Environmental modelling and Software [230].

\subsection{Identifying the potential influences of socio- economic activity factors on pollution}

The annual records regarding five categories: manufacturing, construction, pollution, household, and traffic at district levels across years are selected across 2001 to 2007 from the HK government. Each of the categories is consisted of various variables(indicators) as shown in Appendix 1 . The records of meteorological and pollution for each monitoring station are averaged by year. Then, the average values of the corresponding monitoring station will represent the pollution and meteorological levels at the district where the monitoring station is located. After integrating with data 
from the five socio-economic activity categories, the correlation analysis and panel data analysis will be conducted in the next two subsections.

\subsubsection{Correlation analysis}

Strong correlation for some input pairs could strongly influence the regression models accuracy. Therefore, before the panel data regression analysis, the feature selection will be conducted. High correlation variables will be removed from the candidate variables corresponding to different categories. Figure 5-1to 5-4 display the input correlation for each input categories. The selected ones are marked by using blue text.

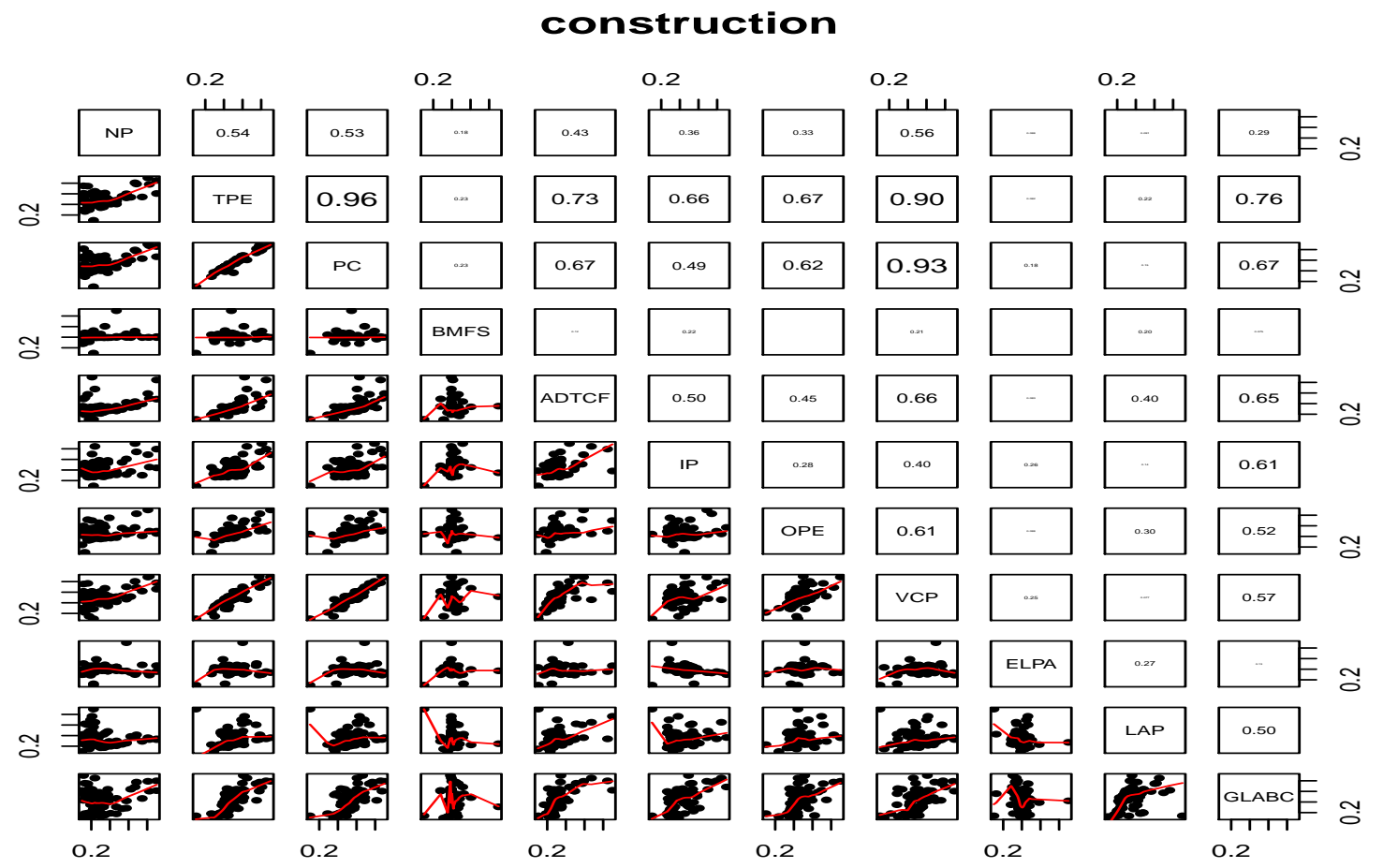

Figure 5-1: The inputs correlation for industry category.

\subsubsection{Pattern discovery}

Hausman test results are taken in Table 5.3, showing that the RE (Table 5.2) model should be chosen as the $\mathrm{P}$ value are larger than 0.05 . This illustrates that the industry 
manufaturer

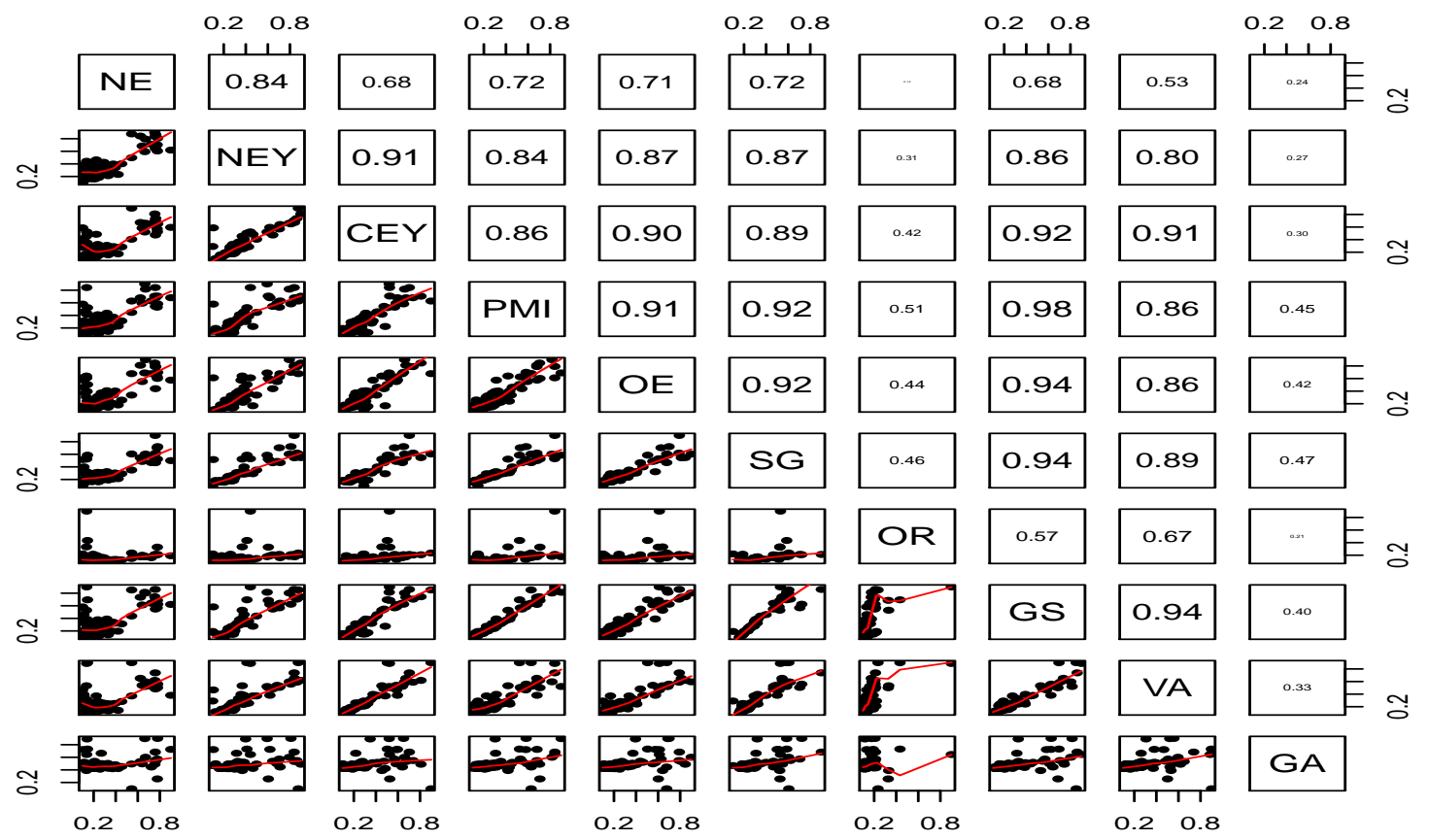

Figure 5-2: The inputs correlation for manufacturing category.

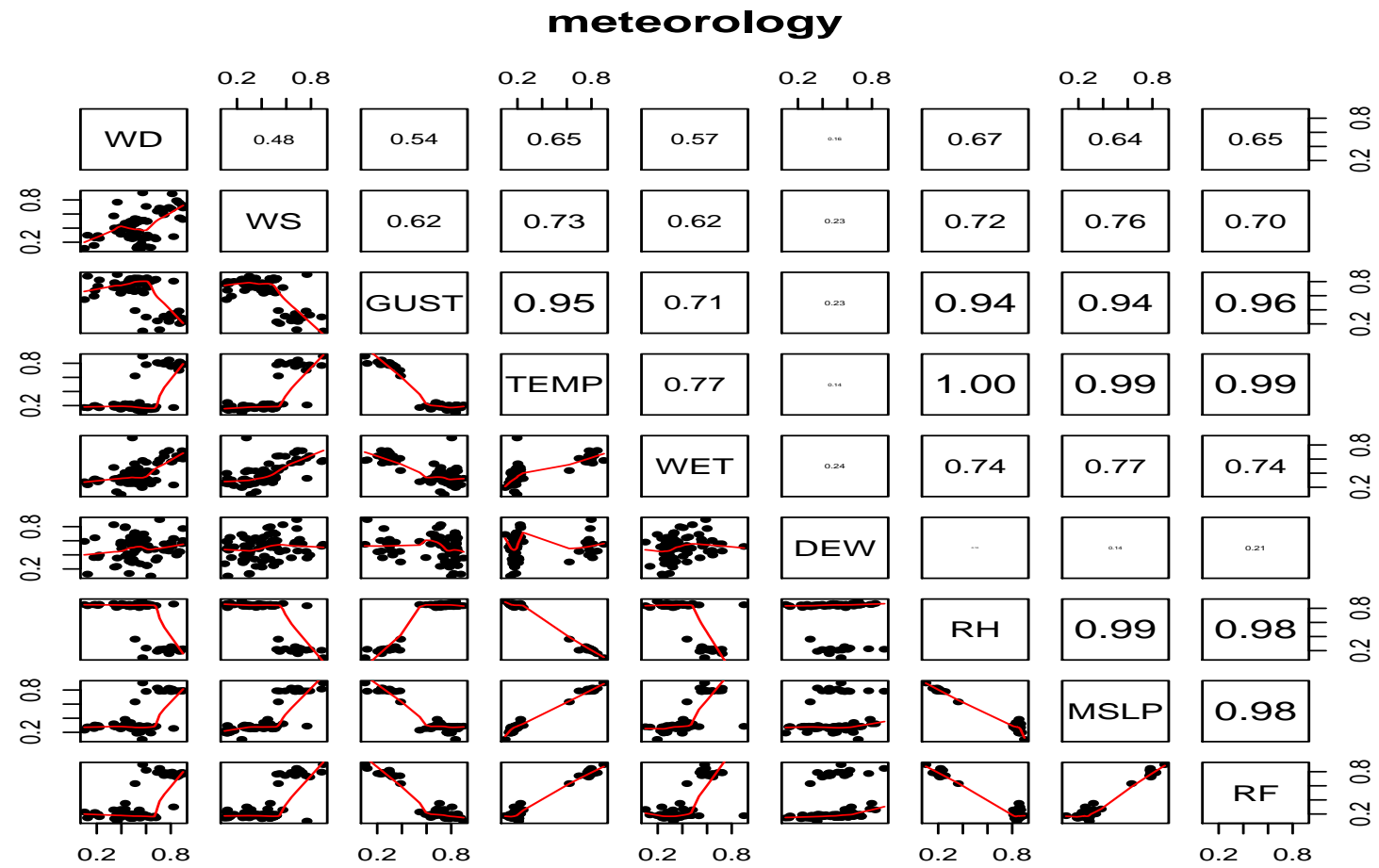

Figure 5-3: The inputs correlation for Meteorology categories. 
Table 5.1: Fixed effected model

\begin{tabular}{|c|c|c|c|c|c|}
\hline & $\mathrm{NO}_{x}$ & $\mathrm{NO}_{2}$ & $\mathrm{SO}_{2}$ & $\mathrm{RSP}$ & $\mathrm{O}_{3}$ \\
\hline \multirow[t]{2}{*}{ R square } & within $=0.8642$ & 0.8368 & 0.4889 & 0.7654 & 0.6931 \\
\hline & overall $=0.8758$ & 0.8119 & 0.5215 & 0.6488 & 0.6646 \\
\hline $\mathrm{NE}$ & $0.513^{* *}$ & $0.705 * * *$ & 0.376 & 0.274 & 0.146 \\
\hline $\mathrm{CEY}$ & -0.466 & $-0.780^{*}$ & -0.284 & -0.214 & -0.052 \\
\hline OR & -0.049 & -0.273 & -0.091 & -0.129 & 0.138 \\
\hline GA & -0.171 & -0.163 & 0.153 & -0.14 & 0.054 \\
\hline VA & 0.176 & 0.453 & -0.322 & -0.107 & 0.045 \\
\hline NP & $0.274^{* *}$ & $0.346^{* *}$ & -0.205 & 0.143 & -0.046 \\
\hline $\mathrm{PC}$ & $-0.340^{*}$ & $-0.472^{* *}$ & 0.252 & -0.318 & 0.14 \\
\hline BMFS & -0.006 & -0.03 & -0.47 & -0.148 & -0.002 \\
\hline $\mathrm{ADTCF}$ & -0.168 & -0.226 & -0.299 & -0.154 & -0.217 \\
\hline IP & -0.139 & -0.078 & -0.125 & -0.236 & 0.055 \\
\hline OPE & 0.248 & $0.335^{*}$ & 0.169 & $0.450 * *$ & -0.074 \\
\hline ELPA & $-0.545^{* * *}$ & -0.273 & 0.286 & 0.063 & -0.019 \\
\hline LAP & $-0.447 * * *$ & $-0.416 * *$ & 0.33 & -0.054 & $0.247^{*}$ \\
\hline POCG & 0.003 & -0.045 & $-0.527^{* *}$ & -0.084 & $0.340^{* *}$ \\
\hline MMHI & 0.052 & -0.086 & 0.282 & 0.047 & 0.025 \\
\hline $\mathrm{HZ}$ & $-0.508^{* * *}$ & $-0.502^{* * *}$ & -0.115 & $-0.230^{*}$ & -0.002 \\
\hline $\mathrm{TF}$ & $0.229^{*}$ & -0.06 & -0.063 & 0.031 & $-0.256^{* *}$ \\
\hline WD & $0.552^{* * *}$ & $0.513^{* * *}$ & -0.175 & $0.229^{*}$ & $0.282^{* * *}$ \\
\hline WS & 0.123 & $0.236^{*}$ & 0.277 & $0.481 * * *$ & -0.137 \\
\hline DEW & -0.092 & -0.166 & $-0.400^{* *}$ & $-0.361^{* * *}$ & -0.043 \\
\hline WET & $0.446^{* * *}$ & $0.376^{* *}$ & 0.272 & 0.165 & 0.155 \\
\hline _cons & $0.576 * * *$ & $0.683^{* * *}$ & $0.761^{* * *}$ & $0.274^{* *}$ & 0.182 \\
\hline heteroskedasticity & No & No & $N O$ & $\mathrm{NO}$ & $\mathrm{NO}$ \\
\hline
\end{tabular}

Note: t-statistic using heteroskedasticity robust estimates

${ }^{*}$ p-value significant at $\alpha=0.05$

${ }^{* *} \mathrm{p}$-value significant at $\alpha=0.01$ 
Table 5.2: Random effected model

\begin{tabular}{|c|c|c|c|c|c|}
\hline & $\mathrm{NO}_{x}$ & $\mathrm{NO}_{2}$ & $\mathrm{SO}_{2}$ & RSP & $\mathrm{O}_{3}$ \\
\hline \multirow[t]{2}{*}{ R square } & Within $=0.8633$ & 0.8282 & 0.4847 & 0.713 & 0.684 \\
\hline & Overall $=0.8783$ & 0.8315 & 0.529 & 0.702 & 0.6919 \\
\hline NE & $0.486^{* *}$ & $0.631 * * *$ & 0.334 & 0.169 & 0.188 \\
\hline CEY & -0.401 & $-0.604^{*}$ & -0.184 & 0.032 & -0.152 \\
\hline OR & -0.054 & -0.288 & -0.099 & -0.15 & 0.147 \\
\hline GA & -0.182 & -0.193 & 0.136 & -0.182 & 0.072 \\
\hline VA & 0.191 & 0.495 & -0.298 & -0.048 & 0.021 \\
\hline NP & $0.295^{* *}$ & $0.402^{* * *}$ & -0.173 & 0.221 & -0.078 \\
\hline $\mathrm{PC}$ & $-0.349^{*}$ & $-0.496^{*}$ & 0.238 & -0.352 & 0.154 \\
\hline BMFS & 0.012 & 0.017 & -0.443 & -0.082 & -0.029 \\
\hline $\mathrm{ADTCF}$ & -0.166 & -0.221 & -0.296 & -0.146 & -0.22 \\
\hline IP & -0.131 & -0.058 & -0.114 & -0.208 & 0.044 \\
\hline OPE & 0.221 & 0.261 & 0.127 & $0.347^{*}$ & -0.033 \\
\hline ELPA & $-0.529 * * *$ & -0.23 & 0.311 & 0.125 & -0.043 \\
\hline LAP & $-0.450 * * *$ & $-0.424^{* *}$ & 0.325 & -0.066 & $0.252^{*}$ \\
\hline POCG & 0.046 & 0.07 & $-0.461^{* *}$ & 0.077 & $0.274^{* *}$ \\
\hline MMHI & 0.006 & -0.212 & 0.21 & -0.129 & 0.096 \\
\hline $\mathrm{HZ}$ & $-0.480^{* * *}$ & $-0.426^{* * *}$ & -0.072 & -0.124 & -0.045 \\
\hline $\mathrm{TF}$ & $0.214^{*}$ & -0.1 & -0.086 & -0.024 & $-0.234^{*}$ \\
\hline WD & $0.552^{* * *}$ & $0.513^{* * *}$ & -0.176 & 0.228 & $0.282^{* * *}$ \\
\hline WS & 0.143 & $0.290^{* *}$ & $0.308^{*}$ & $0.557 * * *$ & $-0.168^{*}$ \\
\hline DEW & -0.083 & -0.141 & $-0.386^{* * *}$ & $-0.327 * * *$ & -0.057 \\
\hline WET & $0.417 * * *$ & $0.298^{*}$ & 0.227 & 0.056 & $0.199^{*}$ \\
\hline _cons & $0.547^{* * *}$ & $0.605^{* *}$ & $0.716^{* *}$ & $0.502^{*}$ & 0.226 \\
\hline heteroskedasticity & No & No & NO & $\mathrm{NO}$ & $\mathrm{NO}$ \\
\hline
\end{tabular}

Note: t-statistic using heteroskedasticity robust estimates

${ }^{*}$ p-value significant at $\alpha=0.05$

${ }^{* *}$ p-value significant at $\alpha=0.01$ 
Population and Household

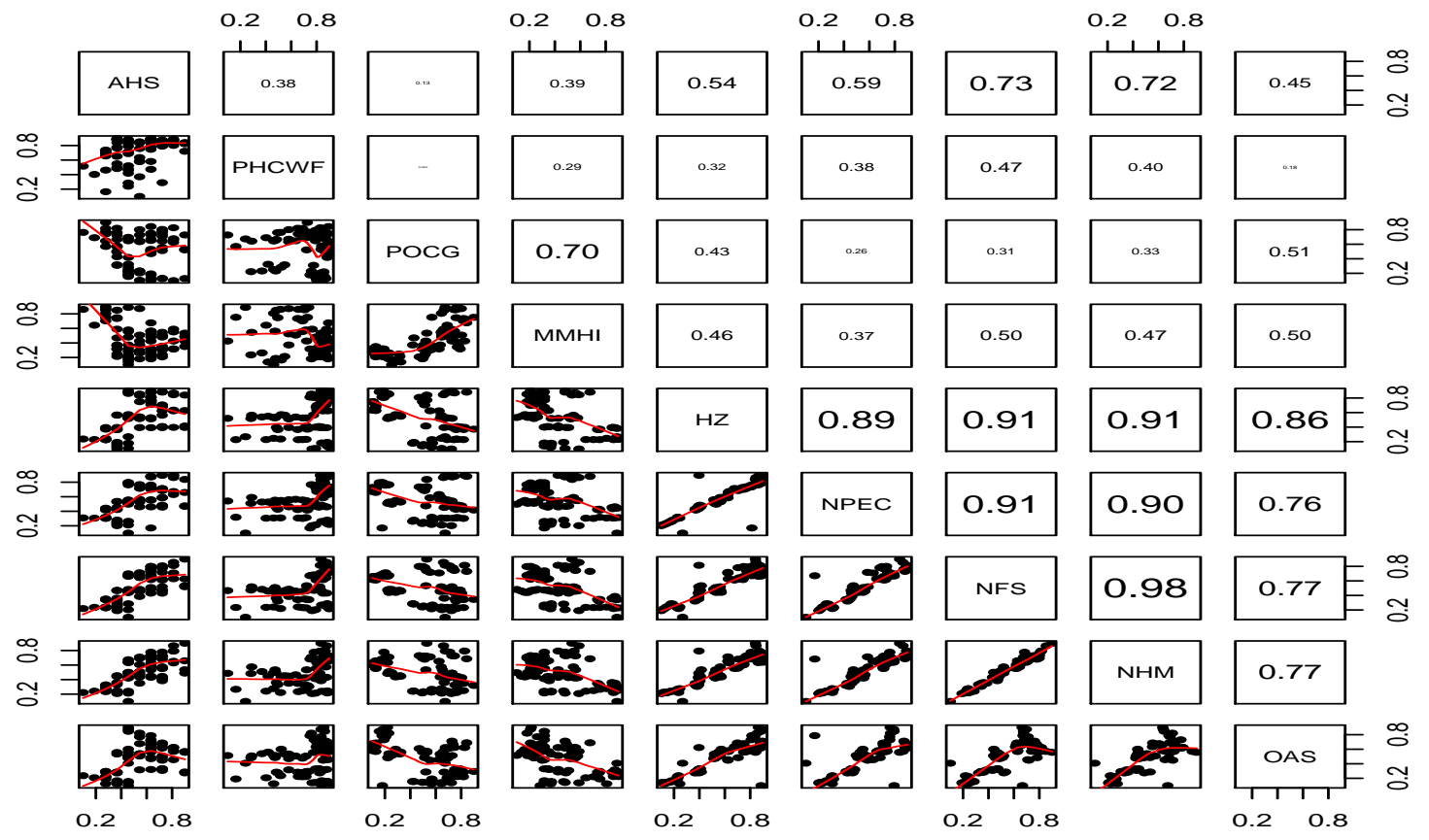

Figure 5-4: The inputs correlation for population and household categories.

Table 5.3: Hausman test

\begin{tabular}{llllll}
\hline & $\mathrm{NO}_{x}$ & $\mathrm{NO}_{2}$ & $\mathrm{SO}_{2}$ & $\mathrm{RSP}$ & $\mathrm{O}_{3}$ \\
\hline $\mathrm{NOx}$ & 1 & & & & \\
$\mathrm{NO}_{2}$ & & 0.9998 & & & \\
$\mathrm{SO}_{2}$ & & & & & \\
$\mathrm{RSP}$ & & & & 0.9484 & \\
$\mathrm{O}_{3}$ & & & & & 1 \\
\hline
\end{tabular}


Table 5.4: Fixed effects model with year dummy

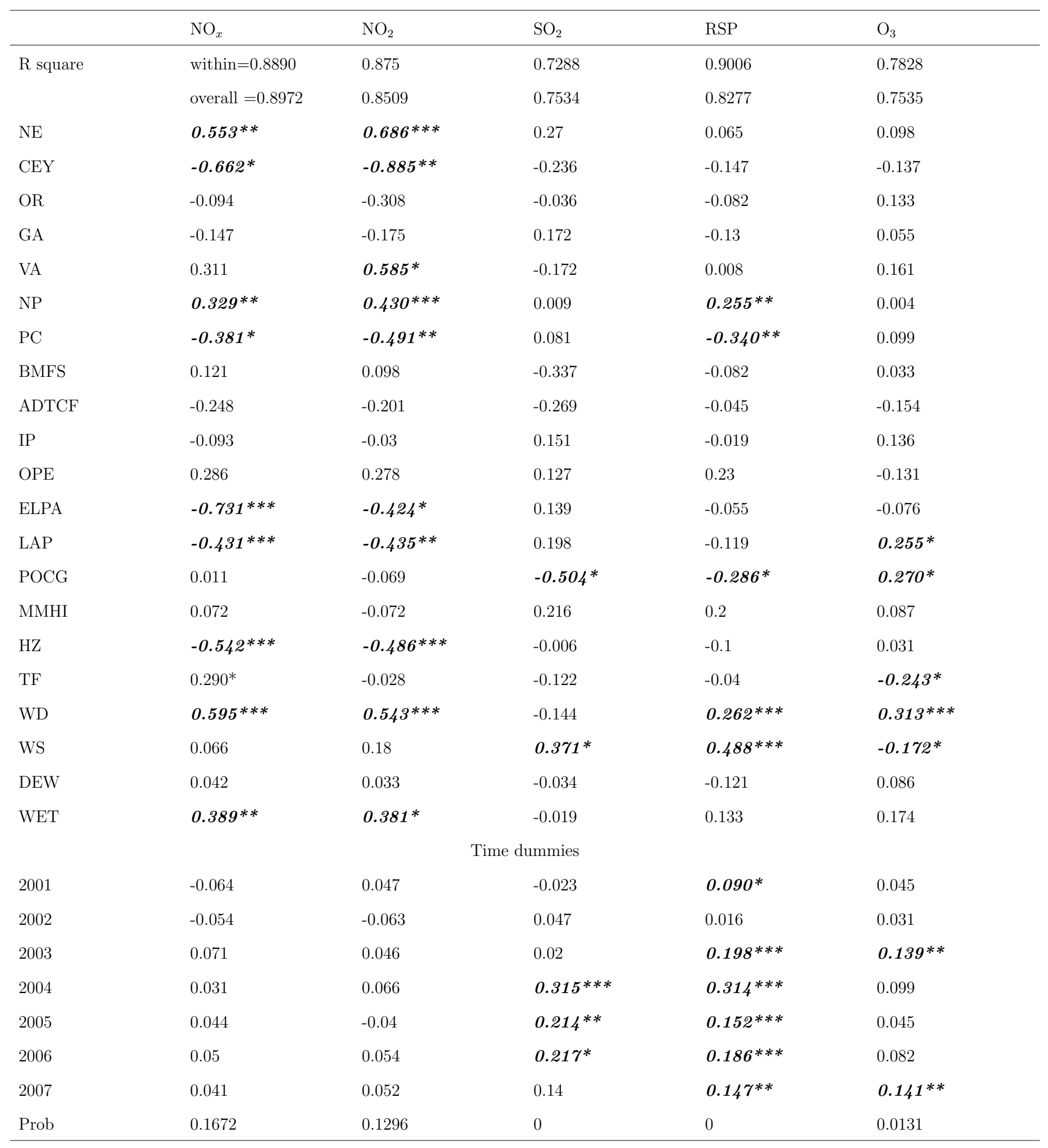

Note: t-statistic using heteroskedasticity robust estimates

${ }^{*}$ p-value significant at $\alpha=0.05$

${ }^{* *}$ p-value significant at $\alpha=0.01$ 
estate area effect could not significantly affect the pollution. However, comparing the analysis results of Table 5.2 and Table 5.1, it can be seen that the differences of estimated coefficient in FE and RE are trivial.

Broadly speaking, the models of $\mathrm{NO}_{x}, \mathrm{NO}_{2}$ can be explained larger than $80 \%$ by the selected input variables for both RE and FE, RSP is $76 \%$ for $\mathrm{EF}$ and $71 \%$ for RE, followed by $\mathrm{O}_{3}, 69 \%$ and $68 \%$ for $\mathrm{FE}$ and $\mathrm{RE}$, indicating that the pollutants $\mathrm{NO}_{x}$, $\mathrm{NO}_{2}$, RSP and $\mathrm{O}_{3}$ are mainly from the sectors manufacturing, construction, transportation, household structure and meteorology in HK. However, these inputs only count for less than $50 \%$ variances of $\mathrm{SO}_{2}$. The main contributors of $\mathrm{SO}_{2}$, therefore, could be from other sources. According to the HK government, electricity generation from power plants accounts for $47 \%$ of the $\mathrm{SO}_{2}$. Due to the limitation of data from power plants, this study, however does not include this sector.

In detail, the variance in $\mathrm{NO}_{x}$ is substantially explained by the fixed effect model ( $\mathrm{R}$ square $=86.42 \%)$ and the random effect models $(86.3 \%)$. The estimated result of $\mathrm{NO}_{2}$, as a major component of nitrogen oxides $\left(\mathrm{NO}_{x}\right)$, is similar with $\mathrm{NO}_{x}$, except for the explanatory variables TF and ELPA. The coefficients on NE, NP, TF, WD, and WET in $\mathrm{NO}_{x}$ model are positive at the $5 \%$ significant level. PC, ELPA, LAP, and HZ have negative coefficients in both random affected and fixed affected model. WD has the strongest positive effect on $\mathrm{NO}_{x}$, while ELPA has the strongest negative effect. The positive coefficients on of NE, NP, TF and WD are consistent with our expectations, which is the $\mathrm{NO}_{x}$ pollution increases as the number of manufacturer establishments, construction projects, and traffic flow grow. Wind direction is an important factor of $\mathrm{NO}_{x}$ level. This is largely due to the fact that the pollution is formatted locally but also is influenced by its nearby environmental situation. The coefficients on $\mathrm{CEY}$ of both $\mathrm{EF}$ and $\mathrm{RF}$ in $\mathrm{NO}_{2}$ models are significantly negative. For $\mathrm{NO}_{x}, \mathrm{SO}_{2}, \mathrm{RSP}$ and $\mathrm{O}_{3}$, the estimates of coefficients are negative too. This reveals the fact the higher the payment to employees, the lower the pollution, as the high salary industries normally refer to the intelligence-intensive industry rather than labor or resource-intensive industry with relatively high pollution. HZ is short for household size. According to the definition of HK government, domestic household consists of a 
group of persons who live together and make common provisions essential for living. It can be an indication of residential area. The higher value of $\mathrm{HZ}$ in one district means it likely to be a residential area rather than industrial or commercial area. Therefore, lower pollution occurred in the areas where the higher HZ is.

Regarding $\mathrm{O}_{3}$, the meteorological factors wind speed and dew are at $1 \%$ significance level. Finally, LAP, POCG, TF, WD and WS are the main factors that influence $\mathrm{O}_{3}$ level. There are two possible reasons. One is that different from the $\mathrm{NO}_{x}$ and $\mathrm{NO}_{2}$ models, traffic low has a considerably negative coefficient on the variable traffic low. Because ozone is formed by the reaction of its precursors, namely nitric oxide emission, under certain weather conditions. Thus, the heavy traffic areas with high $\mathrm{NO}_{x}$ and $\mathrm{NO}_{2}$ concentration levels, normally, have lower ozone levels compared to the areas with low traffic flow. Another reason is that since wind speed and wind direction are important factors for ozone formation, the ozone transportation probably is an other reason for the local high level formation.

The most striking finding of this study is not only the growth of variables, such as number of establishments, number of projects and traffic flow, have a positive and significant association with pollution increasing, which is consistent with our expectations, but also the coefficients of other variables, like CEY, PC, ELPA, LAP, and HZ that reflect the structure of sectors have a strong relation with some of the pollutants. Some of these variables even capture more variances of pollutants.

The results of FE model with year dummies (Table 5.1.2) indicate that there are no time-fixed effects of $\mathrm{NO}_{x}$ and $\mathrm{NO}_{2}$, but there are for RSP, $\mathrm{SO}_{2}$, and $\mathrm{O}_{3}$. These pattern reflects that no significant increase of $\mathrm{NO}_{x}$ and $\mathrm{NO}_{2}$ concentration levels can be found over the years 2001 to 2007, but a considerable rise on time dummies cross years 2004 to 2006 for $\mathrm{SO}_{2}$ model, years 2001, 2003 to 2007 for RSP model, and year 2003 and 2007 for $\mathrm{O}_{3}$ model. Particularly, in 2004, the estimate coefficients are higher on the year dummies for $\mathrm{RSP}$ and $\mathrm{SO}_{2}$ than other years. This pattern largely corresponds to the identifiable episodes with respect to weather conditions. The tropical cyclone in the Western Pacific Ocean near Taiwan is more prevalent in 2004 than any other years. This hot weather condition favors the accumulation 
of RSP and $\mathrm{O}_{3}$ in Hong Kong. However, with the constriction of $\mathrm{NO}_{x}$ and $\mathrm{NO}_{2}$ pollution, $\mathrm{O}_{3}$ does not show an upward trend in 2004, as the year dummy in 2004 is not significant.

\subsection{Research flow for forecasting}

Another objective of this chapter is to apply preprocessing and ensemble artificial intelligence classifiers to forecast daily maximum ozone threshold exceedances in the Hong Kong area. Preprocessing methods, including over-sampling, under-sampling, and the synthetic minority over-sampling technique, were employed to address the imbalance data problem. Ensemble algorithms are proposed to improve the classifier's accuracy. Moreover, a distance-based regional data set was generated to capture ozone transportation characteristics. The data sources and target station selection can be found in Chapter 2, Section 2.1.2. The details of the utilized AI techniques and proprocessing tools are described in Chapter 2, Section 2.2.3. Furthermore, this chapter will also advise on the relative importance of the different variables for ozone pollution prediction and test if the regional data facilitate better forecasting. For comparison, two ways regression are used: One is to use time series for prediction; the other is used the save AI techniques in regression mode with tunning parameters to predict exceedances. The Research flow is illustrated in Figure 5-6.

\subsection{Description analysis}

Even though these efforts have been taken, there are still some types of pollution exceeding the air quality limitation set up by HK government and showing upward trends from 1999 to 2007. Figure 5-6 demonstrate the average value of the pollutants $\mathrm{O}_{3}$ and RSP at different monitor stations across years 1999 to 2007 projecting on google map.

The diurnal variations in the average ozone level in July and October, at the TW, TC, KC, and YL monitoring stations are shown in Figure 5-7. The ozone 


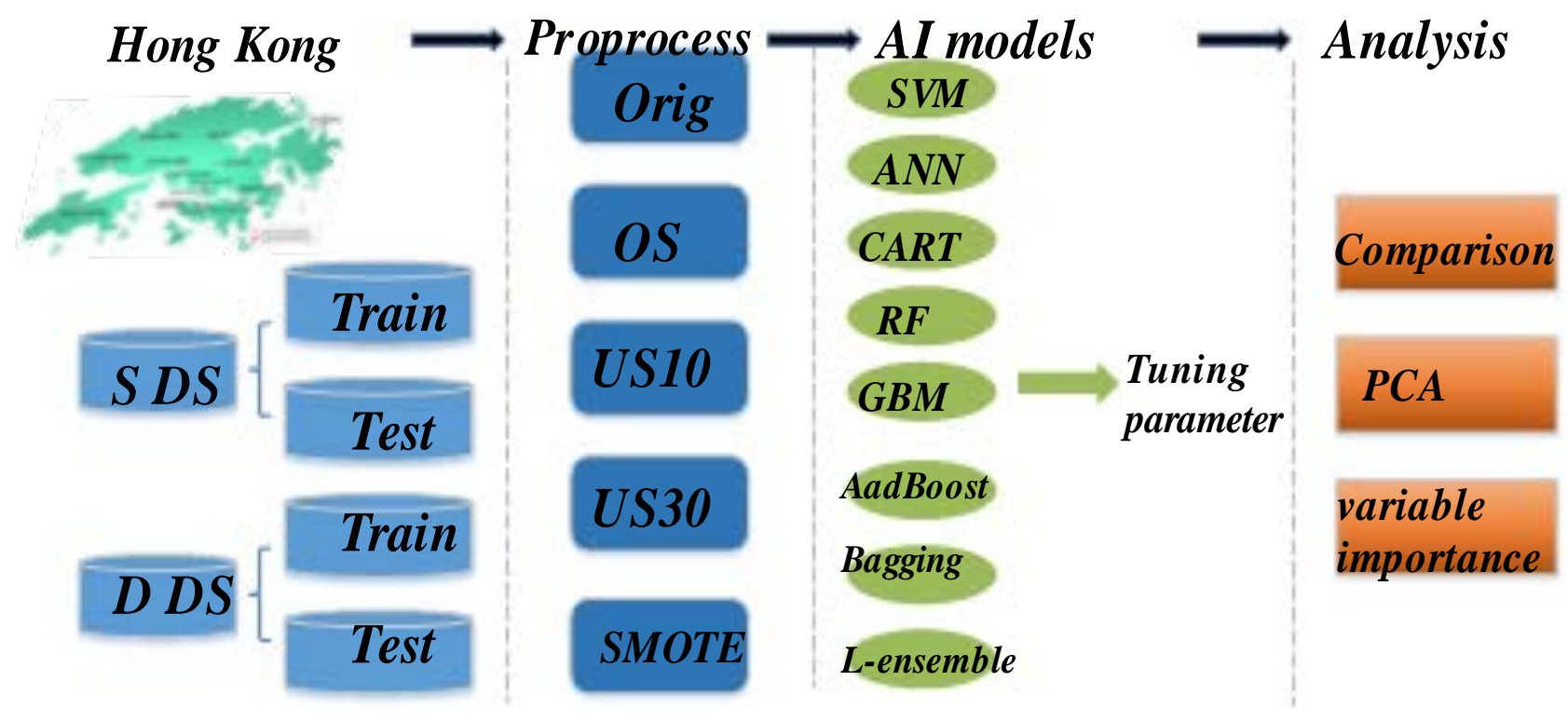

Figure 5-5: Abstract of the case study

concentration levels at these stations demonstrate a consistent pattern in July, which is characterized by one peak in mid-afternoon, between 14:00 and 16:00, and two valleys between 06:00 and 08:00 and after 20:00, remaining stable at a low level from 00:00 to 05:00. In October, the ozone levels increased significantly at these four stations. However, the pattern observed during this period was different from that observed in July, when one peak appeared during the day. In October, two peaks appeared: from 03:00 to 06:00 and from 14:00 to 17:00.

Figure 5-8 shows the frequency of the days on which the daily maximum ozone level occurred at the corresponding time of the day. The $x$ axis represents the time of day and the $y$ axis represents the frequency at which the daily maximum ozone occurred at the corresponding time. Although the average ozone level recorded during all the years was lower in the morning than in the afternoon, there were still many days on which the maximum daily ozone level could be observed between 00:00 and 05:00 at TW and KC. At YL and TC, the frequencies were as high in the morning as in the afternoon. Since previous studies have shown that a high level of ozone concentration is associated with photochemical production from local emissions under certain weather conditions, such as maximum solar radiation, high temperature, or low humidity [72, 30], the high ozone level in the morning with low temperature and 


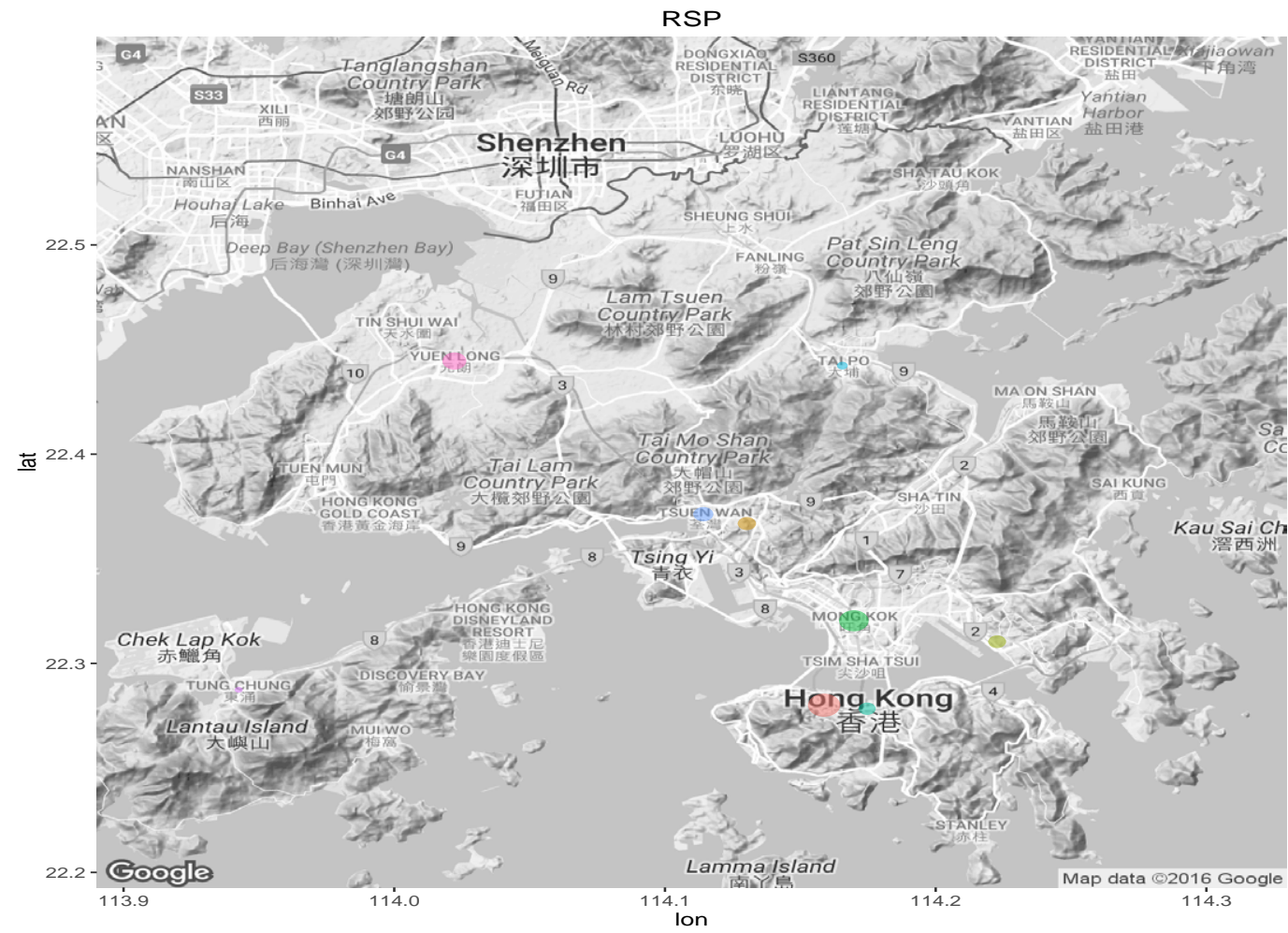

\section{RSP \\ - 56 \\ 60 \\ 64 \\ 68}

pollution.station

- Central

- Kwai Chung

- Kwun Tong

- Mong kok

- Shan Shui Po

- Tai Po

- Tsuen Wan

- Tung Chun

- Yuen Long

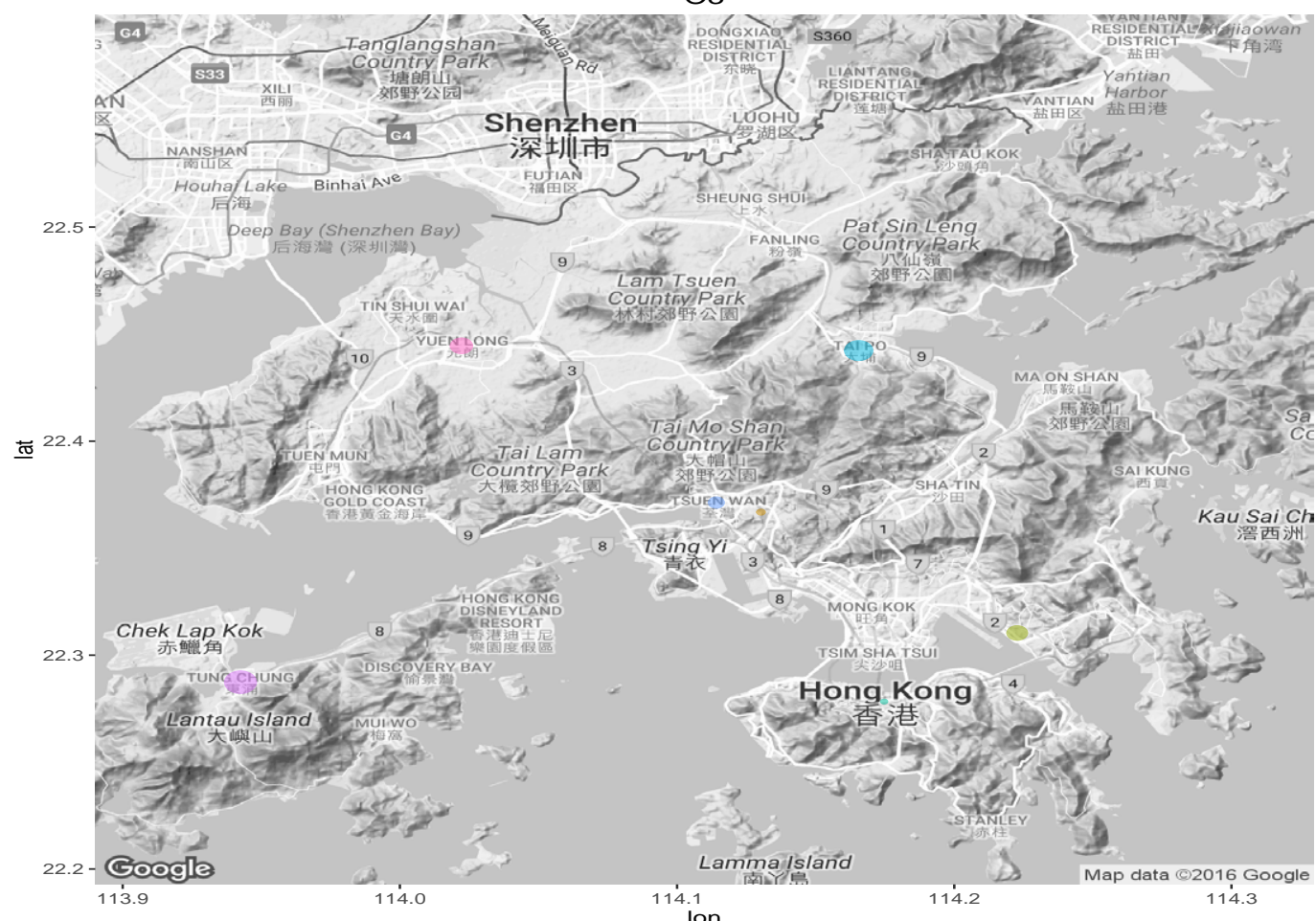

$\mathrm{O} 3$

- 25

○ 30

35
40

pollution.station

- Central

- Kwai Chung

- Kwun Tong

- Mong kok

- Shan Shui Po

- Tai Po

- Tsuen Wan

- Tung Chun

- Yuen Long

Figure 5-6: Average level of $\mathrm{O}_{3}, R S P$ from year 1999 to 2007 . 


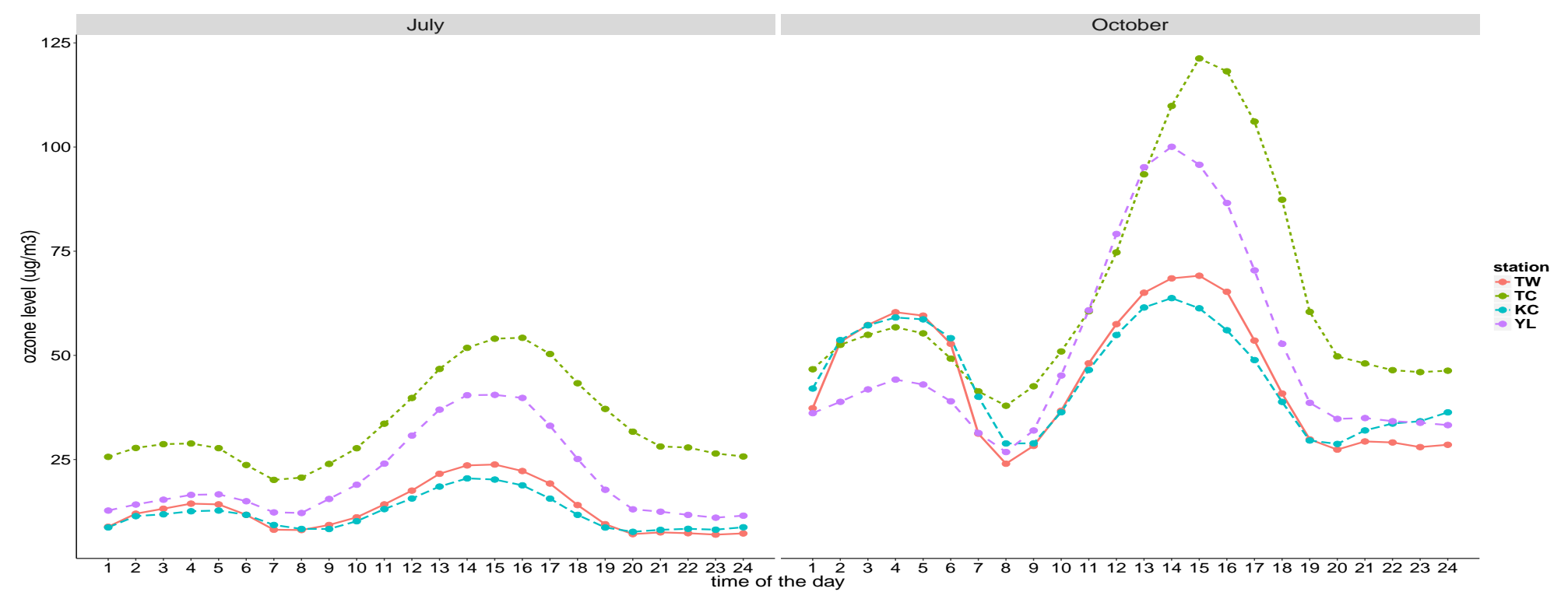

Figure 5-7: Diurnal average ozone concentrations in Hong Kong at selected stations.

high humidity at TW and KC should not be attributed to the local emissions, but rather to ozone transportation, as discussed in the introductory part.

The ubiquity of local ozone results from the reaction of its precursors, such as $N O_{x}$, $\mathrm{NO}_{2}$, and $\mathrm{VOC}$, under certain meteorological conditions. These precursors, however, are the consequences of anthropogenic emissions associated with transportation, industry activities, biomass burning, fossil fuel refinement, and distribution [231].

Figure 5-9 shows that the number of industries is not consistent with the $\mathrm{O}_{3}$ threshold exceedances at the local area (district) level in HK. For example, the number of $\mathrm{O}_{3}$ exceedances in Tusen Wan (TW) and Kwai Chung (KC), which contain the highest number of manufacturing establishments, is relatively low. Conversely, the pollution levels in areas with a small number of manufacturers, such as Yue Long (YL) and Sha Tin (ST), are high.

Furthermore, HK is near one of the most developed areas, the Pearl River Delta (PRD), in China. The pollution from this remote emission source probably contributes to the local ozone formation. Figure 5-10 presents the hourly $\mathrm{O}_{3}$ concentration levels from October $5^{\text {th }}$ to October $7^{\text {th }}, 2008$ at the TW and YL stations. YL is the nearest monitoring station to the PRD area. During this period, the wind direction at the YL station was between 270 and 315 degrees. The distance between 

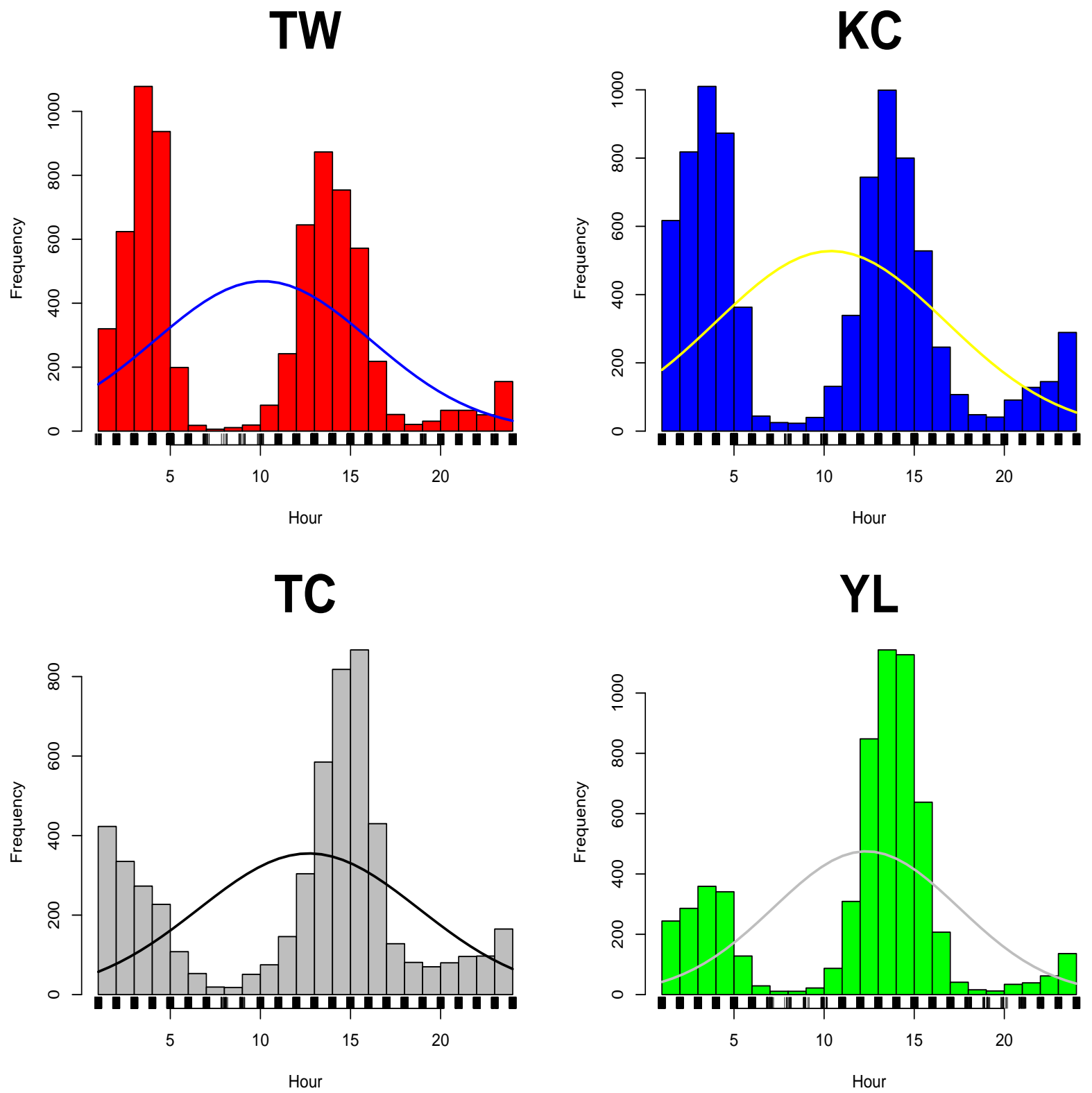

Figure 5-8: Frequency at which the daily maximum ozone appears at the corresponding time at the selected monitoring stations. 


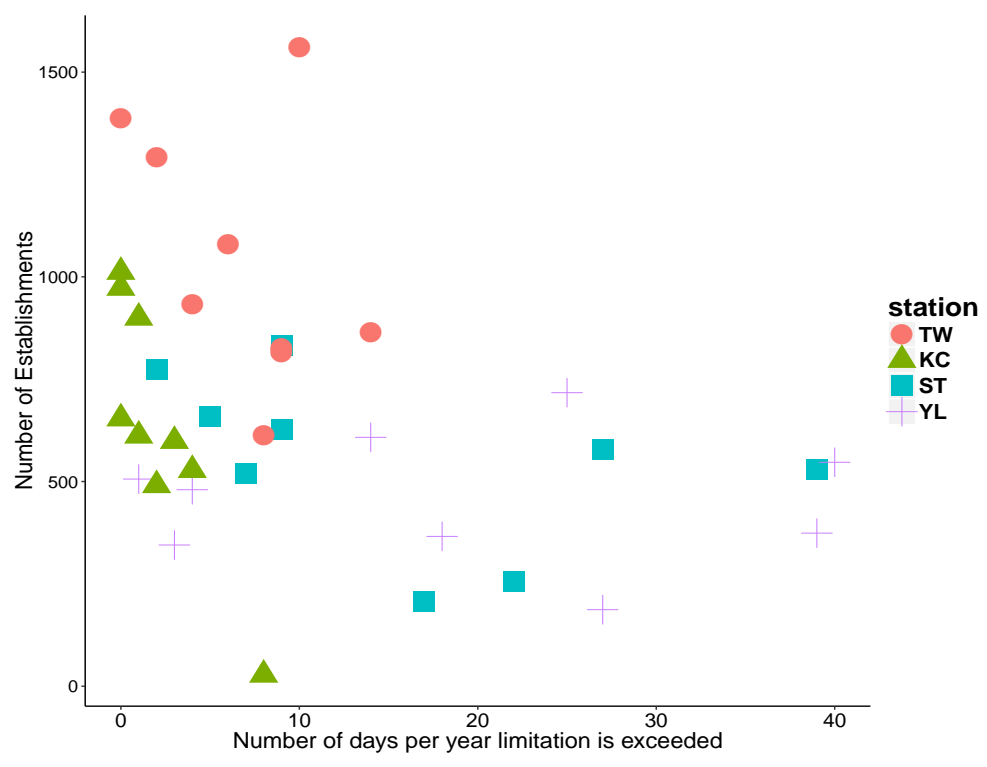

Figure 5-9: Number of days on which the limitation was exceeded versus the number of manufacturers from 1999 to 2007 (data sources: Census and Statistics Department of HK government)

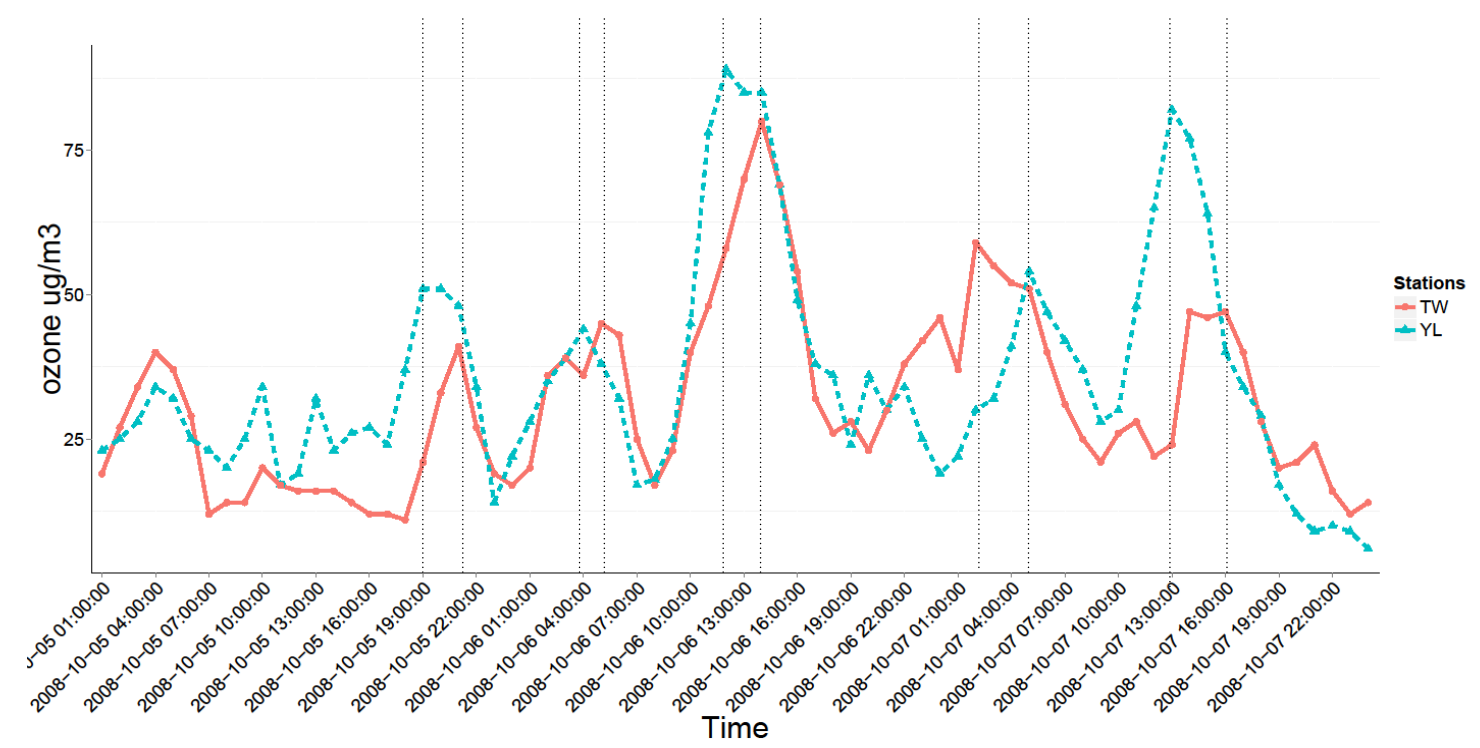

Figure 5-10: $\mathrm{O}_{3}$ levels at TW and YL stations from 2008-10-05 to 2008-10-07. 
TW and YL is $12.5 \mathrm{~km}$. The maximum wind speed was $11 \mathrm{~m} / \mathrm{s}$, the minimum was $0.7 \mathrm{~m} / \mathrm{s}$, and the average was around $5 \mathrm{~m} / \mathrm{s}$. In Figure $5-10$ it can be seen that the time of the $\mathrm{O}_{3}$ peak level at the YL station is one to two hours in advance of that at the TW station. Therefore, the main responsibility for $\mathrm{O}_{3}$ formation at TW is $\mathrm{O}_{3}$ transportation rather than the local industrial activities at TW, which means that the pollution from industry in local areas in $\mathrm{HK}$ is not the only source for local $\mathrm{O}_{3}$ formation. Thus, global factors, such as $\mathrm{O}_{3}$ transportation, could play a significant role in $\mathrm{O}_{3}$ pollution forecasting.

The variables for the daily maximum or minimum levels of ozone precursors, including $\mathrm{O}_{3}, \mathrm{NO}_{x}$, and $\mathrm{NO}_{2}$ from the previous day, the ratio of $\mathrm{NO}_{x} / \mathrm{NO}_{2}$ and meteorological factors, such as temperature $(T M P)$, relative humidity $(R H)$, wind speed $(W S P)$, and wind direction $(W D R)$, as well as description variables, such as season $(S)$, month $(M)$, and weekday $(W)$ were considered as candidate inputs.

To provide a fair comparison, in this study the distance-based data set was prepared first. After merging all the variables listed in Table 2.4, a total of 2149 samples was obtained in the distance-based data set. Then, the input features from only the TW station were extracted from the distance-based data set to generate the singlesite data set (Table 2.1.1). The single-site data set consists of 18 inputs and the distance-based set contains 22 input variables.

Eighty percent of the samples in both data sets were used for training and validation and $20 \%$ for testing. There are two types of output, one for regression mode, which is continuous values, and the second for classification mode with categorical variables. Regarding the classification, the output is labeled "H" or "L." "H" means the ozone concentration is higher than the limitation $160 \mu \mathrm{g} / \mathrm{m}_{3}$; otherwise it is labeled "L." Finally, 1719 samples were obtained for training and validation, 1681 of which were "L" and 38 were "H." Meanwhile, 430 samples were used for testing with 12 samples "H" and 418 "L."

The new variables were normalized by Equation 5.1

$$
\text { NewVariable }=\frac{\text { variable }-\min (\text { variable })}{\max (\text { variable })-\min (\text { variable })}
$$




\section{$5.4 \quad$ Forecasting}

\subsubsection{Classification forecasting performance}

The main steps of the method are shown in Figure 5-11. 1) The preparation of the two data sets, namely, single-site and distance-based, are described in Chapter2, Section 2.1.2. 2) Then, the preprocessing methods and cross validation technique are introduced in Chapter2, Section 2.2.2 and Section 2.2.5, respectively. 3) In addition, the AI techniques and their corresponding parameter tuning are described from Chapter2, Section 2.2 .3 and Section 2.2.4. 4) Finally, the performance measurements for evaluation are presented in Section 2.2.6. In this section, the model performance results

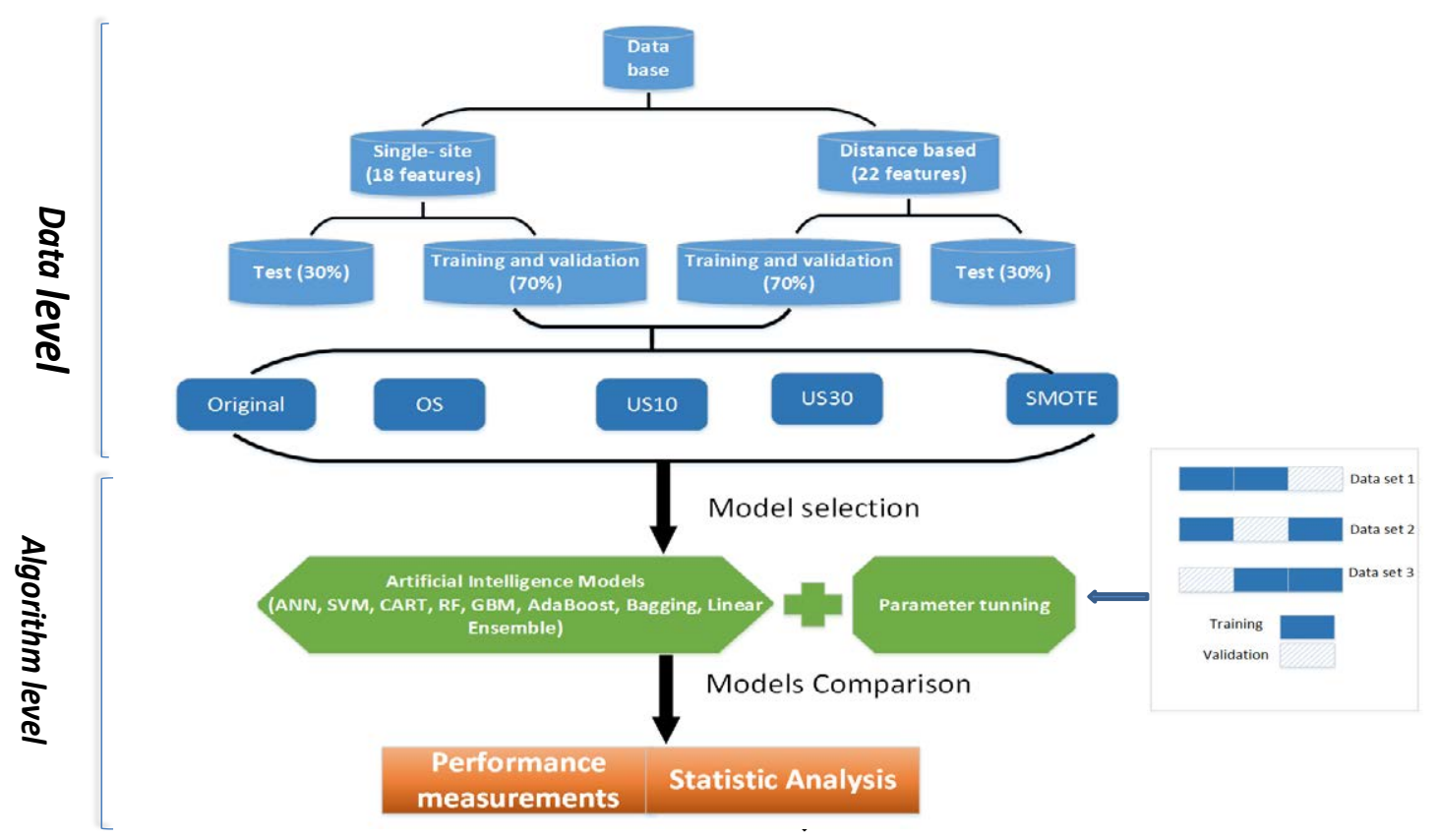

Figure 5-11: Methodology overview.

for each combination of re-sampling and AI methods are compared in terms of the three performance measurements: $G_{\text {mean }}$, weighted accuracy $W A$, and $F_{\text {measure }}$. The comparison is presented in two forms: vertical and horizontal. The vertical comparison involves fixing a particular AI technique and comparing the results obtained from 
various re-sampling methods; the horizontal comparison involves an AI comparison analysis based on a specific re-sampling method.

Table 5.5 shows the classification performance based on the single-site and distancebased data sets. The values in italic font are the best performances for each AI technique, while the values in bold font are the best for each re-sampling method.

Regarding the single-site data set, the vertical comparison reveals that SVM, ANN, CART, RF, GBM, Adaboost, and L-Ensemble with US30 yield more accurate models according to $G_{\text {mean }}$ and WA than these classifiers with the original data set and other re-sampling methods. For Bagging, the best model was achieved by US10. According to $F_{\text {measure, }}$, the AI techniques SVM, RF, and Bagging combined with US10 outperform the respective techniques with OS, US30, and SMOTE. It can be concluded that the US method are noteworthy among the preprocessing methods. According to the horizontal comparison, where the preprocessing method is fixed, the CART-based single and ensemble algorithms (CART, GBM, AdaBoost CART, and Bagging) can achieve better results than ANN and SVM, in general.

Similarly, comparisons are also presented based on the distance-based data set. Vertical comparison reveals that US30 is the best preprocessing data technique for SVM, ANN, RF, and Adaboost according to $G_{\text {mean }}$ and WA. According to $F_{\text {measure }}$, US10 is the best preprocessing method for all the AI techniques, except CART. The horizontal comparison reveals that ensemble classifiers constitute the ideal methods for enhancing model performance; in particular, the Adaboost algorithm significantly improves the performance for the original data set, OS, US10, and US30.

Finally, a comparison of the models for the single-site and distance-based data sets is presented. More accurate forecasting results can be achieved by the distance-based data set than by the single-site data set. Therefore, a preliminary conclusion can be drawn: the regional factors are of importance in ozone level prediction. A variable importance analysis is presented in the next section in order to verify the importance of the regional factors in this case.

In order to ensure that the results of the assessment were not obtained by chance, we employed the non-parametric Wilcoxon signed-ranks test to verify the hypothesis 
Table 5.5: Classification results corresponding to different sampling methods and AI techniques by adopting both single-site and distance-based data sets.

\begin{tabular}{|c|c|c|c|c|c|c|c|c|c|}
\hline & & SVM & ANN & CART & \multicolumn{2}{|c|}{ GBM } & AdaBoost & Bagging & Linear Ensemble \\
\hline \multicolumn{10}{|c|}{ Single-site data set } \\
\hline \multirow[t]{3}{*}{ Original } & $G_{\text {mean }}$ & - & 0 & 0 & 0.288 & 0.500 & 0.408 & 0.000 & 0 \\
\hline & WA & 0.6 & 0.6 & 0.6 & 0.632 & 0.700 & 0.667 & 0.597 & 0.6 \\
\hline & $F_{\text {measure }}$ & - & - & - & 0.143 & 0.400 & 0.286 & - & - \\
\hline \multirow[t]{3}{*}{ OS } & $G_{\text {mean }}$ & 0 & 0.488 & 0.565 & 0.288 & 0.285 & 0.616 & 0.287 & 0 \\
\hline & WA & 0.6 & 0.671 & 0.707 & 0.632 & 0.619 & 0.714 & 0.625 & 0.6 \\
\hline & $F_{\text {measure }}$ & - & 0.171 & 0.235 & 0.143 & 0.087 & 0.185 & 0.105 & - \\
\hline \multirow[t]{3}{*}{ US10 } & $G_{\text {mean }}$ & 0.498 & 0.493 & 0 & 0.567 & 0.565 & 0.561 & 0.745 & 0 \\
\hline & WA & 0.696 & 0.684 & 0.6 & 0.712 & 0.707 & 0.700 & 0.805 & - \\
\hline & $F_{\text {measure }}$ & 0.333 & 0.231 & - & 0.258 & 0.235 & 0.205 & 0.359 & - \\
\hline \multirow[t]{3}{*}{ US30 } & $G_{\text {mean }}$ & 0.789 & 0.844 & 0.851 & 0.770 & 0.715 & 0.807 & 0.716 & 0.803 \\
\hline & WA & 0.798 & 0.846 & 0.840 & 0.801 & 0.759 & 0.821 & 0.760 & 0.815 \\
\hline & $F_{\text {measure }}$ & 0.196 & 0.241 & 0.198 & 0.242 & 0.197 & 0.237 & 0.200 & 0.225 \\
\hline \multirow[t]{3}{*}{ SMOTE } & $G_{\text {mean }}$ & 0.000 & 0.727 & 0.746 & 0.736 & 0.554 & 0.726 & 0.479 & 0.000 \\
\hline & WA & 0.578 & 0.777 & 0.768 & 0.790 & 0.686 & 0.776 & 0.650 & 0.581 \\
\hline & $F_{\text {measure }}$ & - & 0.241 & 0.180 & 0.286 & 0.163 & 0.237 & 0.120 & - \\
\hline
\end{tabular}

\section{Distance-based data set}

\begin{tabular}{llllllllll}
\hline \hline Original & $G_{\text {mean }}$ & 0 & 0 & 0 & 0 & 0.287 & $\mathbf{0 . 4 0 8}$ & 0.000 & 0 \\
& WA & 0.6 & 0.6 & 0.6 & 0.6 & 0.628 & $\mathbf{0 . 6 6 7}$ & 0.594 & 0.6 \\
& $F_{\text {measure }}$ & - & - & - & - & 0.118 & $\mathbf{0 . 2 8 6}$ & - & - \\
OS & $G_{\text {mean }}$ & 0 & 0.285 & 0.487 & 0.288 & 0.287 & $\mathbf{0 . 6 2 4}$ & 0.286 & 0 \\
& WA & 0.6 & 0.619 & 0.670 & 0.632 & 0.625 & $\mathbf{0 . 7 2 8}$ & 0.620 & 0.6 \\
& $F_{\text {measure }}$ & - & 0.087 & 0.167 & 0.143 & 0.105 & $\mathbf{0 . 2 2 7}$ & 0.091 & - \\
US10 & $G_{\text {mean }}$ & 0.499 & 0.496 & 0.494 & 0.700 & 0.749 & $\mathbf{0 . 8 0 3}$ & 0.693 & 0.692 \\
& WA & 0.697 & 0.690 & 0.686 & 0.789 & 0.810 & $\mathbf{0 . 8 4 7}$ & 0.776 & 0.774 \\
& $F_{\text {measure }}$ & 0.353 & 0.273 & 0.240 & 0.462 & 0.400 & $\mathbf{0 . 4 7 1}$ & 0.343 & 0.333 \\
US30 & $G_{\text {mean }}$ & 0.811 & 0.809 & 0.853 & 0.822 & 0.770 & $\mathbf{0 . 8 6 0}$ & 0.714 & 0.663 \\
& WA & 0.827 & 0.824 & 0.857 & 0.841 & 0.801 & $\mathbf{0 . 8 6 6}$ & 0.757 & 0.728 \\
& $F_{\text {measure }}$ & 0.250 & 0.243 & 0.267 & 0.290 & 0.242 & $\mathbf{0 . 2 9 0}$ & 0.194 & 0.176 \\
SMOTE & $G_{\text {mean }}$ & 0.000 & 0.806 & $\mathbf{0 . 8 9 2}$ & 0.789 & 0.681 & 0.775 & 0.775 & 0.000 \\
& WA & 0.591 & 0.820 & $\mathbf{0 . 8 8 8}$ & 0.826 & 0.757 & 0.808 & 0.808 & 0.594 \\
& $F_{\text {measure }}$ & - & 0.234 & $\mathbf{0 . 2 8 2}$ & $\mathbf{0 . 3 3 3}$ & 0.250 & 0.262 & 0.262 & - \\
\hline \hline
\end{tabular}

Original: without sampling; US10: under-sampling 10;

US30: under-sampling 30; OS: over-sampling;

167

The extreme situation is that all the "H" are predicted as "L." 
of the improved performance of the classifiers. The mean value of the respective measurement for each classifier was calculated based on Table 5.5. Table 5.6 shows the statistic comparison between Models A and Models B based on $G_{m e a n}$,and their corresponding P-value of the Wilcoxon signed-ranks test in the blanket. The ensemble algorithm AdaBoost significantly outperforms all the other classifiers .

Table 5.6: Outcomes of Wilcoxon matched-pairs signed-ranks test for measurement $\mathrm{G}_{-}$mean

\begin{tabular}{|c|c|c|c|c|c|c|c|c|}
\hline \multirow{2}{*}{ Model } & & \multicolumn{7}{|c|}{ B } \\
\hline & & ANN & CART & $\mathrm{RF}$ & GBM & AdaBoost & Bagging & L-Esemble \\
\hline \multirow{8}{*}{ A } & \multirow{2}{*}{ SVM } & 0.2351 & 0.2291 & $0.2652^{*}$ & $0.2796^{*}$ & $0.3992^{* *}$ & $0.2096^{*}$ & -0.0440 \\
\hline & & $(-0.1073)$ & $(-0.1073)$ & $(-0.0174)$ & $(-0.0137)$ & $(-0.0059)$ & $(-0.0423)$ & $(-0.8551)$ \\
\hline & \multirow{2}{*}{ ANN } & & -0.0060 & 0.0301 & 0.0445 & $0.1641 *$ & -0.0255 & $-0.2791 *$ \\
\hline & & & $(-0.234)$ & $(-0.4772)$ & $(-0.7695)$ & $(-0.0322)$ & $(-0.6241)$ & $(-0.0423)$ \\
\hline & \multirow{2}{*}{ RPART } & & & 0.0361 & 0.0505 & 0.1702 & -0.0195 & -0.2730 \\
\hline & & & & $(-1)$ & $(0.625)$ & $(-0.0829)$ & $(-0.4412)$ & $(-0.0760)$ \\
\hline & \multirow{2}{*}{ RF } & & & & 0.0144 & $0.1340^{*}$ & -0.0556 & $-0.3092 *$ \\
\hline & & & & & $(-0.6953)$ & $(-0.0273)$ & $(-0.0756)$ & $(-0.0174)$ \\
\hline & \multirow{2}{*}{ GBM } & & & & & $0.1197^{*}$ & -0.0700 & $-0.3235^{* *}$ \\
\hline & & & & & & $(-0.0195)$ & $(-0.4316)$ & $(-0.0059)$ \\
\hline & \multirow{2}{*}{ AdaBoost } & & & & & & $-0.1896^{*}$ & $-0.4432^{* *}$ \\
\hline & & & & & & & $(-0.0328)$ & $(-0.0059)$ \\
\hline & \multirow{2}{*}{ Bagging } & & & & & & & $-0.2536^{*}$ \\
\hline & & & & & & & & $(-0.0423)$ \\
\hline
\end{tabular}

${ }^{*}$ p-value significant at $\alpha=0.05$

${ }^{* *} \mathrm{p}$-value significant at $\alpha=0.01$

\subsubsection{Regression performance}

\section{AI regression with tuning threshold}

This study also focused on whether the regression mode can forecast the ozone threshold exceedances as the classifiers did. The results of regression prediction based on the distance-based data set for all the algorithms can be seen in Figure 5.8. Then, 
the prediction ozone values are binarized according to a threshold. In order to optimize the classification performance, the original threshold $\left(160 \mu \mathrm{g} / \mathrm{m}^{3}\right)$ is discarded and a new empirical threshold is tuned over the training data set by three-fold cross validation.

In detail, real values on the validation folds are labeled " $\mathrm{H}$ " or " $\mathrm{L}$ " according to the original threshold, while the prediction values are labeled according to the respective tuning threshold. Then, the average classification performance $\left(G_{\text {mean }}\right)$ over the three validation folds can be calculated. The threshold that yields the highest performance is then selected. Table 5.7 demonstrates that a threshold of $0.32\left(78.525 \mu \mathrm{g} / \mathrm{m}^{3}\right.$ in actual ozone value),outperforms the others. Finally, the classification performances of each learning method for the test set can be obtained (Table 5.8). Figure 512 illustrates the threshold 160 and tuned threshold $78.525 \mu \mathrm{g} / \mathrm{m}^{3}$, as well as the prediction value produced by regressors and the actual ozone value.

Rebalancing (preprocessing) and tuning the threshold are two cost-insensitive methods that accommodate the misclassification cost directly. A comparison of the results of these two methods (Table 5.8 and Table 5.5) reveals that the classifier with preprocessing methods, in particular under sampling and SMOTE, outperforms the counterpart regressors with a tuning threshold.

Table 5.7: Averaged $G_{\text {mean }}$ over the three validation sets from distance-based data set corresponding to different thresholds

\begin{tabular}{llllllll}
\hline Threshold & SVM & ANN & CART & RF & GBM & AdaBoost & Bagging \\
\hline 0.26 & 0.6562 & 0.6781 & 0.7241 & 0.6503 & 0.6666 & 0.6564 & 0.651 \\
0.28 & 0.6779 & 0.7308 & 0.7241 & 0.7054 & 0.7164 & 0.7162 & 0.7173 \\
0.3 & 0.6753 & 0.7721 & 0.7479 & 0.7664 & 0.7637 & 0.7594 & 0.7473 \\
0.32 & $\mathbf{0 . 7 0 0 8}$ & $\mathbf{0 . 7 6 3 3}$ & $\mathbf{0 . 7 7 9 8}$ & $\mathbf{0 . 8 0 2 8}$ & $\mathbf{0 . 8 1 4 3}$ & $\mathbf{0 . 8 0 1 4}$ & $\boldsymbol{0 . 8 1 7 6}$ \\
0.34 & 0.6814 & 0.7825 & 0.7562 & 0.7642 & 0.7626 & 0.7764 & 0.8091 \\
0.36 & 0.6177 & 0.805 & 0.7383 & 0.7677 & 0.7697 & 0.7597 & 0.7382 \\
0.38 & 0.5377 & 0.7337 & 0.723 & 0.739 & 0.7402 & 0.7042 & 0.7054 \\
0.4 & 0.4407 & 0.6505 & 0.7118 & 0.6922 & 0.6834 & 0.6474 & 0.6672 \\
\hline
\end{tabular}


Table 5.8: Performances of learning methods by threshold $0.32\left(78.525 \mu \mathrm{g} / \mathrm{m}^{3}\right.$ in actual ozone)

\begin{tabular}{lllllllll}
\hline & SVM & ANN & CART & RF & GBM & AdaBoost & Bagging & L-Ensemble \\
\hline$G_{\text {mean }}$ & 0.7566 & 0.747 & 0.7753 & 0.7542 & 0.7577 & 0.7566 & 0.7589 & 0.7458 \\
WA & 0.7579 & 0.7464 & 0.7809 & 0.755 & 0.7593 & 0.7579 & 0.7608 & 0.745 \\
$F_{\text {measure }}$ & 0.15 & 0.1406 & 0.1731 & 0.1475 & 0.1513 & 0.15 & 0.1525 & 0.1395 \\
\hline
\end{tabular}

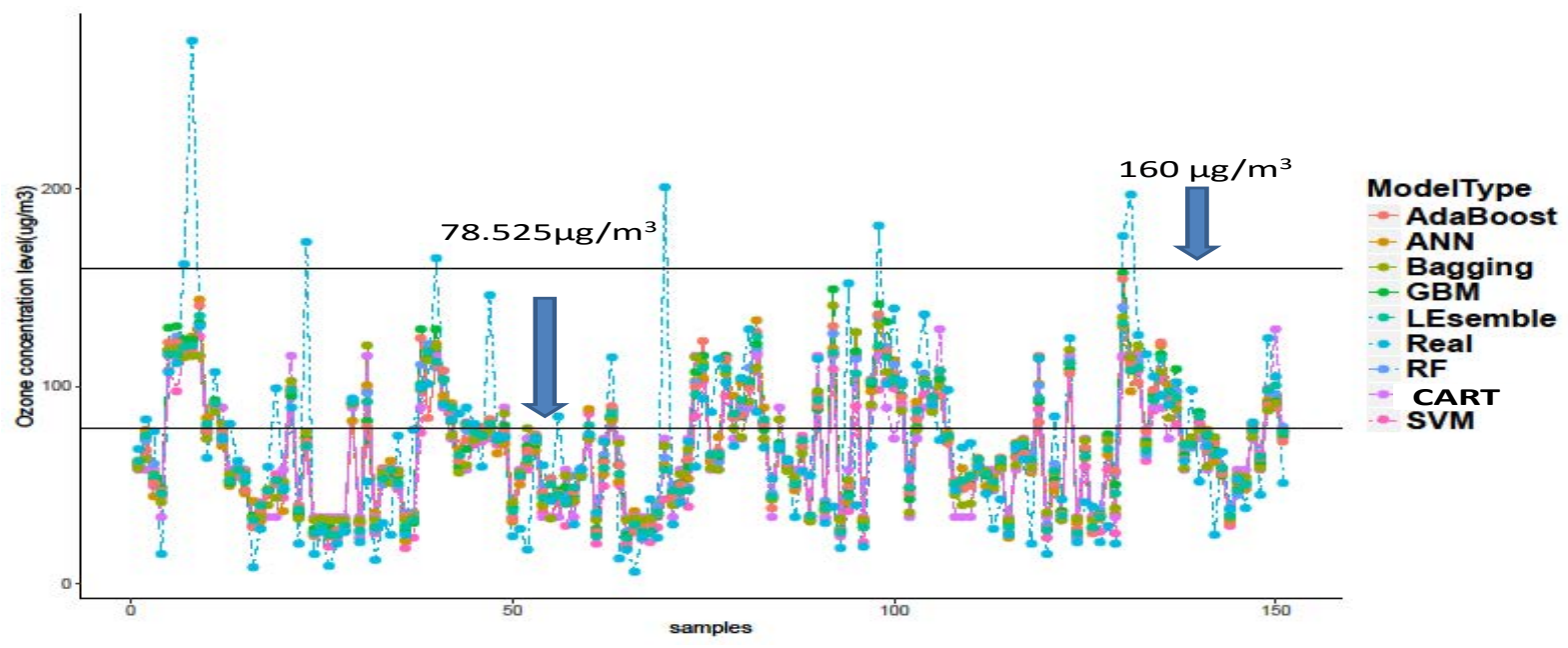

Figure 5-12: Predicted value versus observed value of distance-based data set.

\section{Auto-regressive}

Figure 5-13 shows the trend of ozone concentration levels from 2004 to 2012, which shows that this time series data are probability affected by seasonality and there is a peak an autumn. The three components (seasonal, trend and remainder) were subtracted from the original data (Figure 5-14). The seasonal component changes slowly. Consequently two consecutive years have a similar pattern. The trend component shows fluctuation during this period. The grey bar on the right side of each panel show the relative scales of the components. The large gray bar in the trend panel illustrates the variation in the trend part is much smaller than the variations in the data and other components. Meanwhile, the seasonal component with a media size scale compared to original data showing the variation of original data is partially 
explained by seasonal factors but not much. In contrast, the remainder component has the same level scale as the original part, which gives a strong evidence that the daily maximum ozone levels are explained by other factors, not itself. Therefore, the time series method may not forecast ozone accurately. In order to test it, we still use ARIMA (p,d,q), which is the commonly technique in the time series analysis, to predict ozone. The first lag difference was used for data transformation from nonstationary to stationary. To determine the parameters of ARIMA, ACF and PACF were plotted (Figure 5-16). As there is only one significant spike (at lag one) thereafter for both $\mathrm{ACF}$ and PACF respectively. The structure of $\mathrm{p}=1, \mathrm{~d}=0, \mathrm{q}=1$ will be adopted., where p refers to number so auto-regressive parameters, d stands for the differencing operations performed and $\mathrm{q}$ is moving average parameter. The next 50 days forecasting is shown on Figure 5-17. At the end, the histogram of forecasting errors can be seen in Figure 5-18. It verified the fact that the ozone concentration level cannot be predicted accurately by time series in our study case.

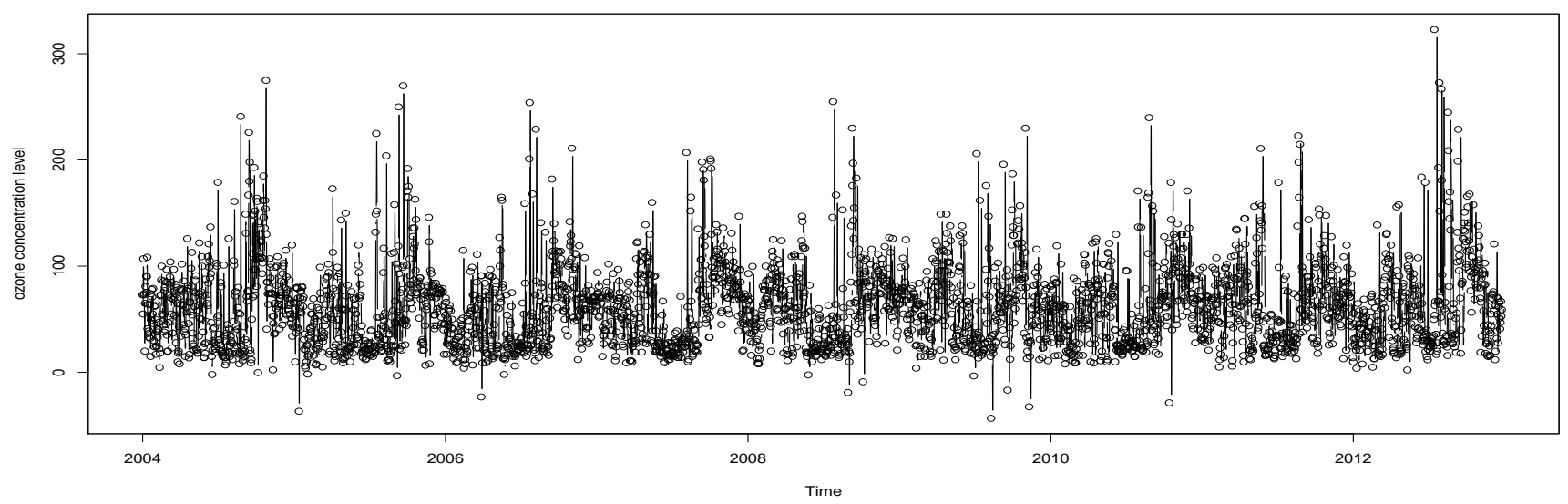

Figure 5-13: Ozone concentration levels from 2004 to 2012.

\subsection{Importance of variables}

In this study, an importance score was applied for evaluating the variables' importance based on four CART-based models. For CART, the variable importance score is 


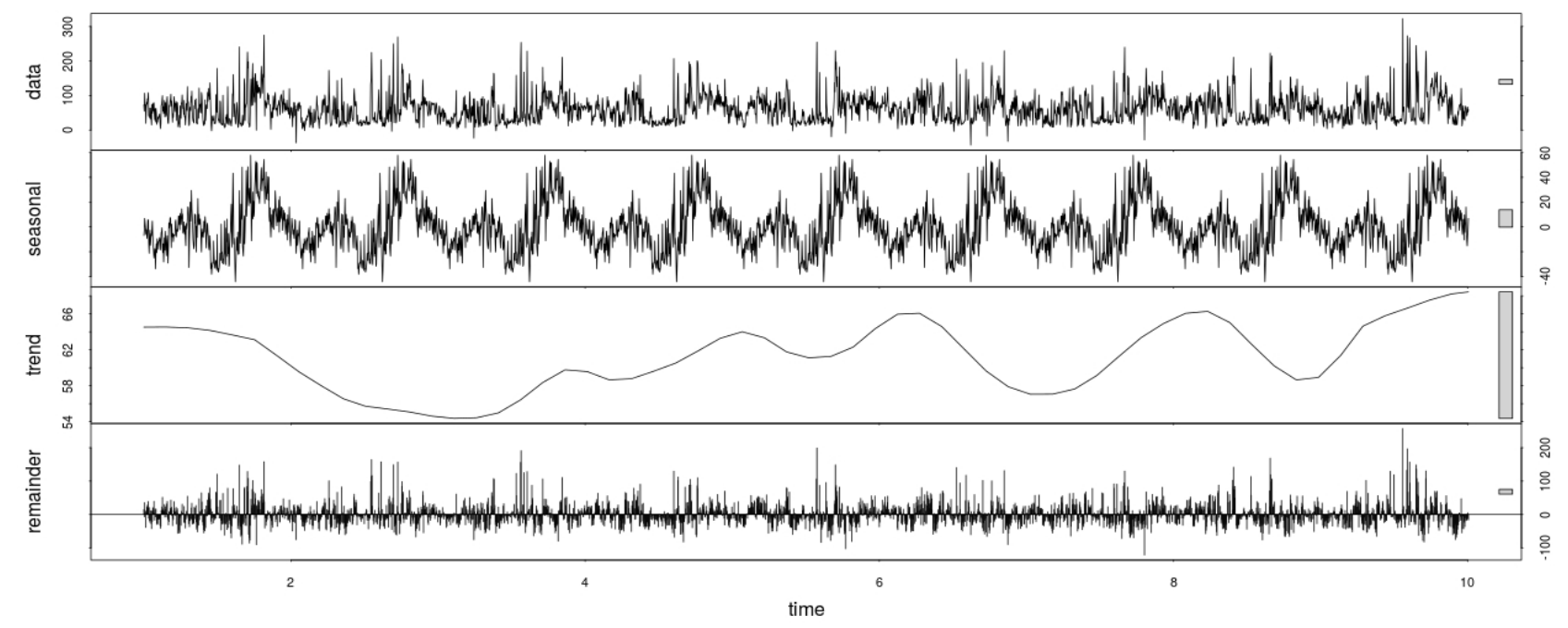

Figure 5-14: The daily maximum ozone levels and its three additive components.

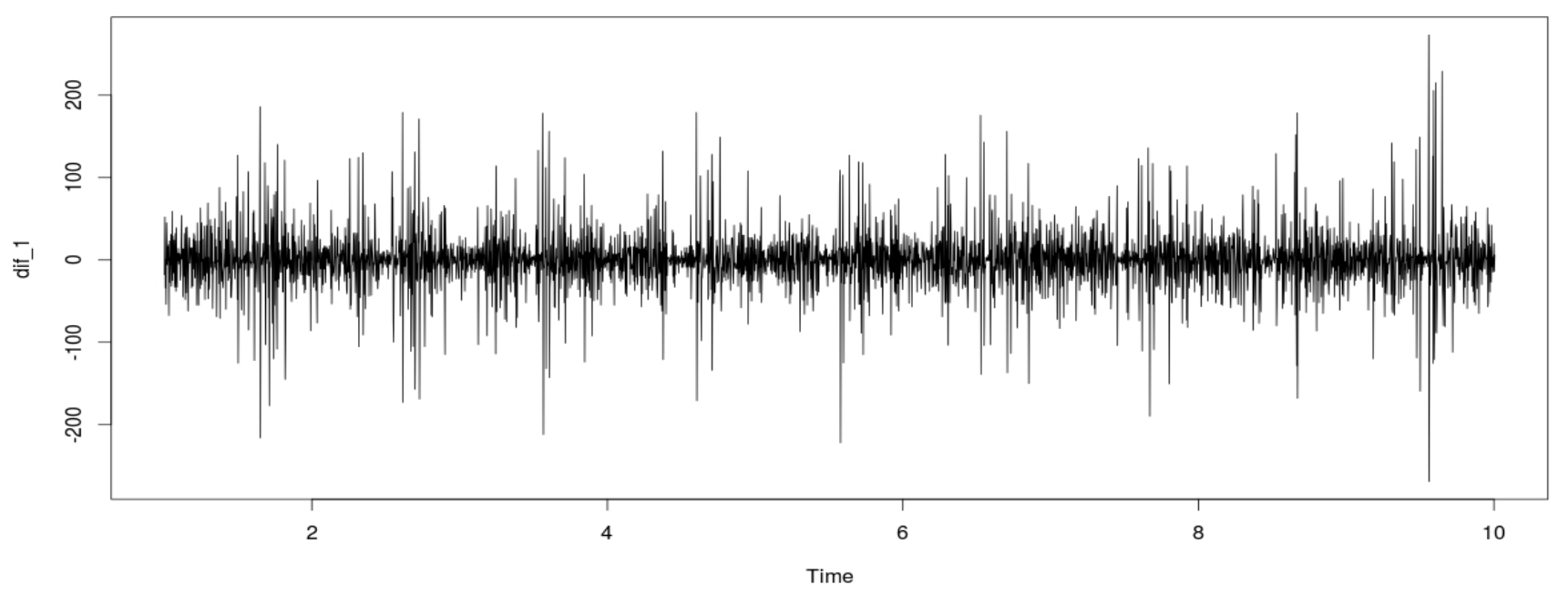

Figure 5-15: Data after one lag differencing. 

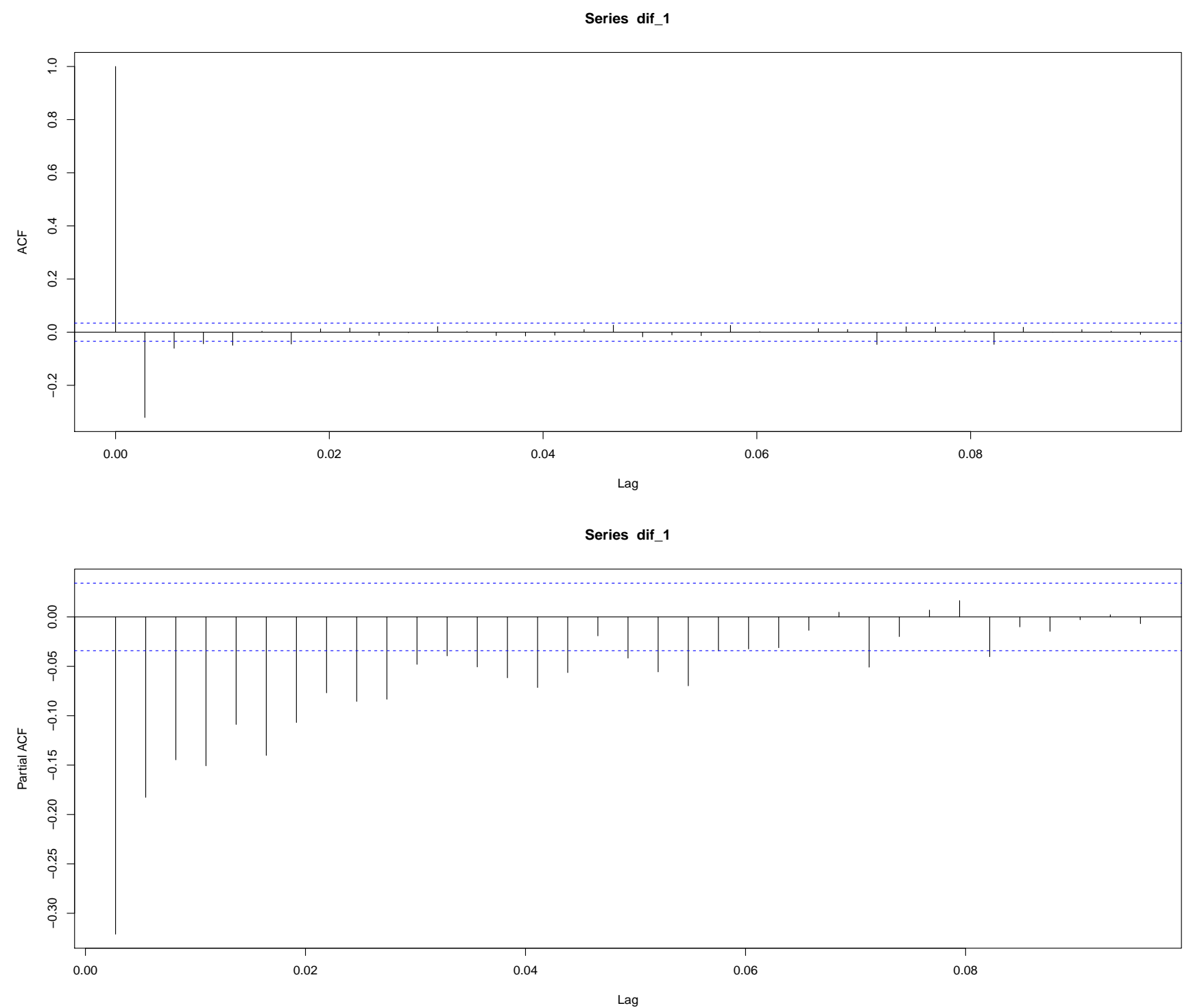

Figure 5-16: $\mathrm{ACF}$ (top) and PACF(bottom) plots for one lag differenced adjusted daily maximum ozone data. 


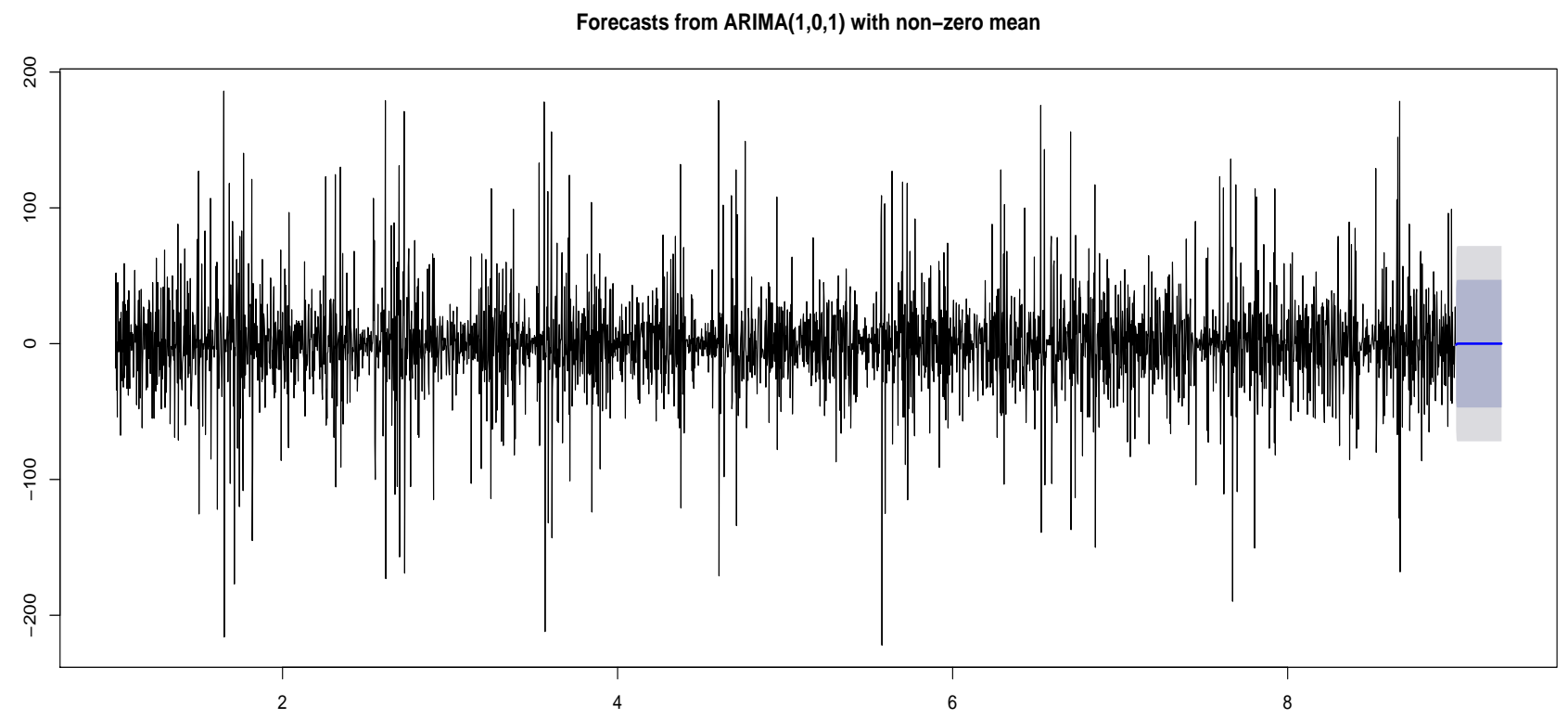

Figure 5-17: Forecasts for the one lag difference adjusted daily maximum ozone level

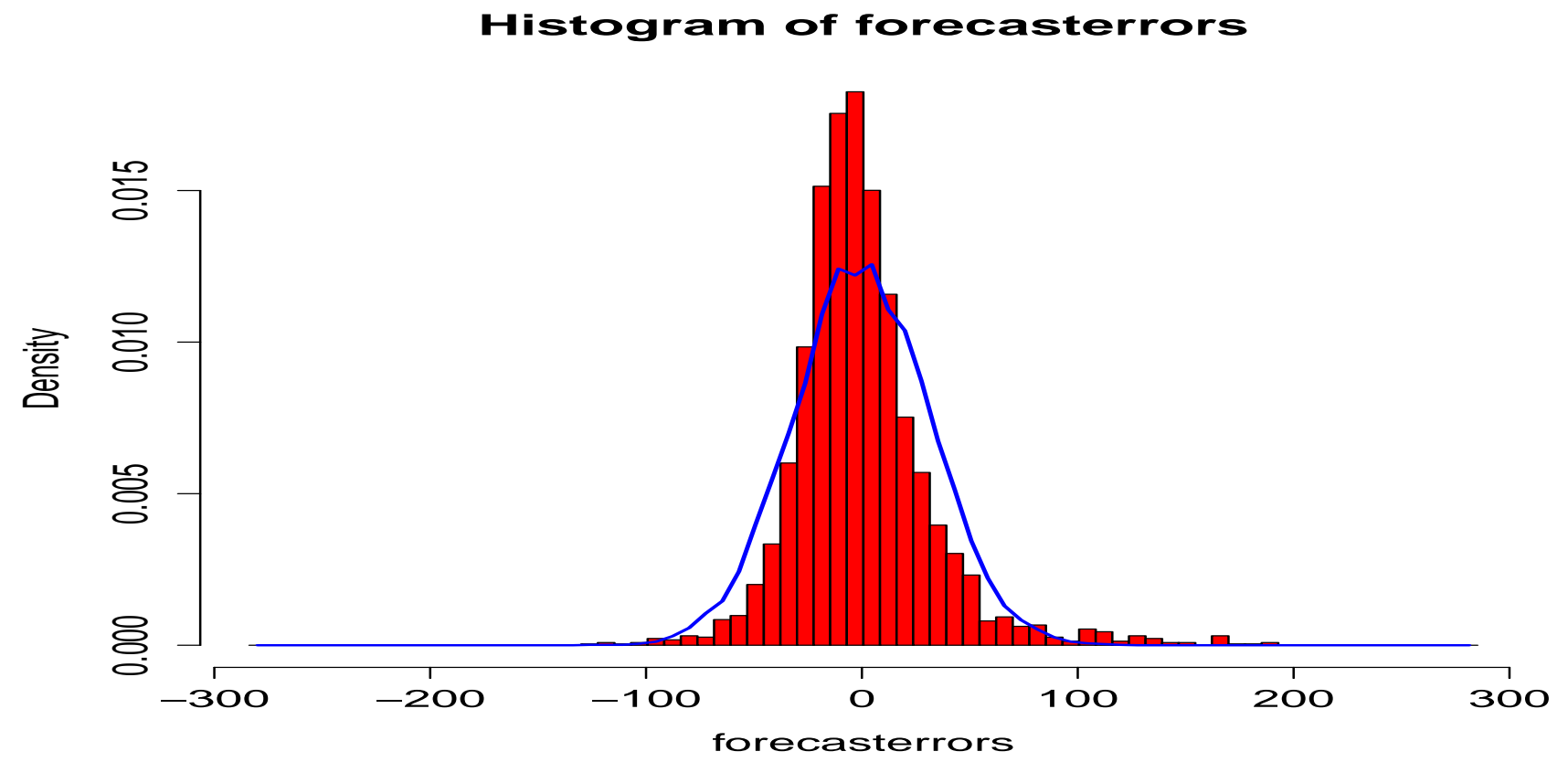

Figure 5-18: Histogram of forecast errors. 
computed based on the contribution of the predictors composing the tree. Specifically, the score is the sum across all the nodes in the tree of the improvement scores when the variable is used as primary or surrogate splitters. In our case, the improvement scores for CART were calculated by the decreasing impurity based on the Gini criterion. As CART is the base learner for the bagging and boosting algorithm in our case, the calculation of the importance scores followed the same pattern, that is, averaging all the decreasing impurity over all three.

Figure 5-19 illustrates the variable importance ranking corresponding to the distancebased data set. In addition to the meteorological and description variables, such as $\operatorname{Max}(T M P)_{p}$ and $\operatorname{Min}(T M P)_{p}, M$, and $S$, that rank as high as those in the singlesite data set, the $\mathrm{O}_{3}$ from the YL, TP, and KC stations of the previous one day domain are the most important factors for the output. The influence degree is from YL, TP, $\mathrm{KC}$ to ST. However, the distance from TW to YL is the longest while to $\mathrm{KC}$ it is the shortest, as shown in Figure 2-5. Thus, the pollution from the nearest monitoring station cannot be the most influential factor on $\mathrm{O}_{3}$ at TW. In other words, ozone concentration from more distant monitoring stations could be more important than that from the nearest ones. In this case, the TP station, located to the northeast of the TW station, is upwind of TW. Meanwhile, the YL station is the nearest station to the PRD area of mainland China, which is one of the most developed in China. Therefore, the "position" factor influences $\mathrm{O}_{3}$ more than the "distance" factor.

\subsection{Discussion}

One of this chapter's targets is to comprehensively assess the influences of socioeconomic activities factors from five categories, namely manufacturer, construction, traffic and transportation, population and household structure and meteorology, on the pollutants $\mathrm{NO}_{x}, \mathrm{NO}_{2}, \mathrm{O}_{3}$, RSP and $\mathrm{SO}_{2}$ respectively at district level in macro environmental Hong Kong. Instead of questionnaire survey a and sampling by measurement emission directly at sampling sites to obtain data, this study uses the statistic data from Hong Kong annual report in different sectors and pollution records from 


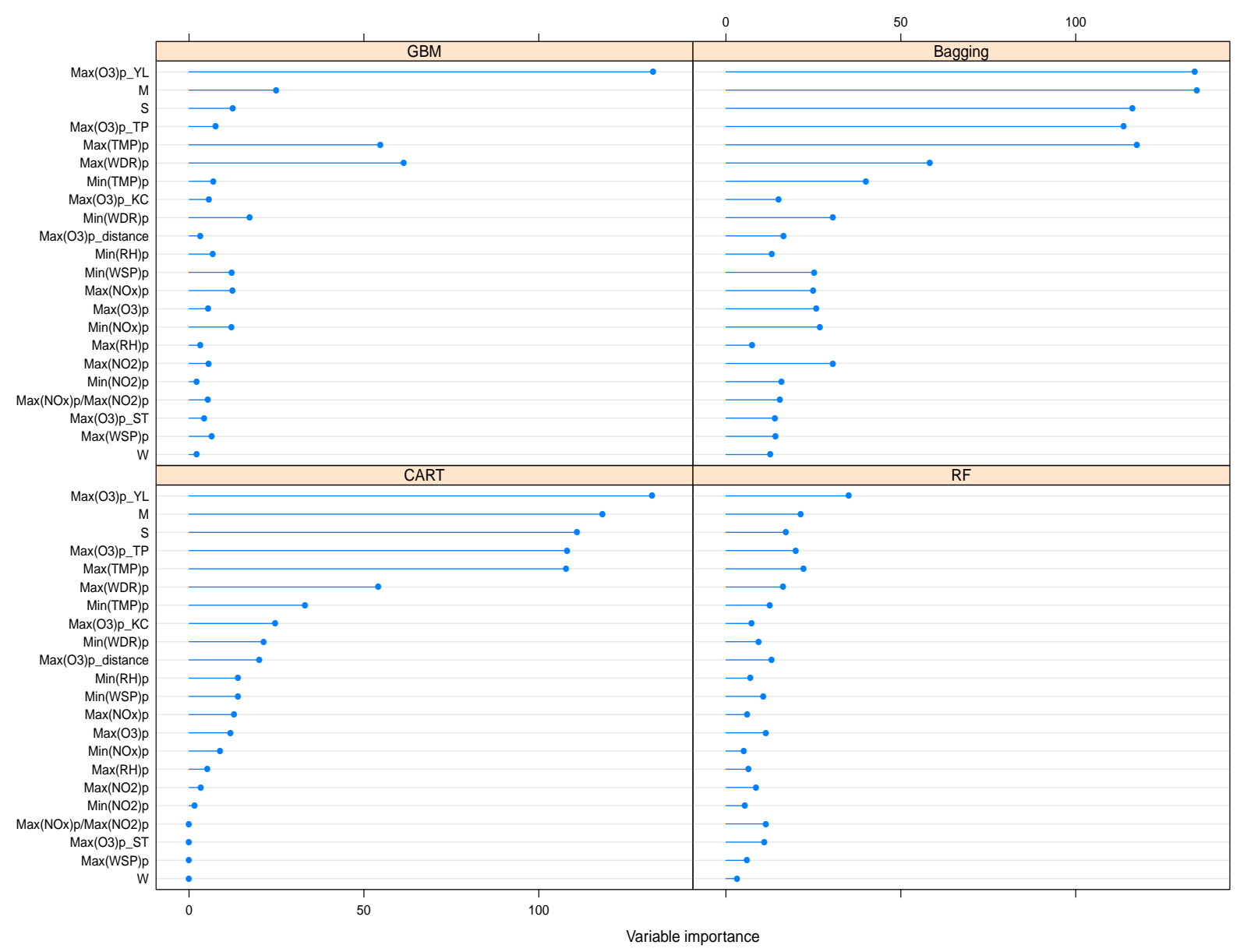

Figure 5-19: Variable importance corresponding to SMOTE combining with CART, GBM, Bagging, and RF classifiers based on distance-based data set.

monitoring stations and adopted econometrics method, fixed-effect model and random effect model, to explore each pollutant formation mechanism from socio-economic activity prospective.

However, there are still some limitations in this work. From the previous studies and the research report of HK government, the electricity generation from power stations plays an import role on pollution. One of the aims of this study is to comprehensively assess the factors on pollution. The lack of the data lead to the missing understanding some important behaviors respect to some pollutants. For example, the lack of electricity generation from the power station is the main contributor to $\mathrm{SO}_{2}$ and RSP, lead to failure of modeling in our case.

Another objective of this study was to forecast the daily maximum ozone thresh- 
old exceedances in the Hong Kong area. A single-site data set and distance-based regional data set were prepared to examine the contributors to local ozone formation. Combinations of preprocessing methods and both single and ensemble AI methods were employed to improve the forecasting accuracy. In addition, the regression mode with tuning threshold was applied for comparison to the classification mode. Finally, variable importance analysis was used to provide further empirical evidence of the main factors of local ozone formation.

The results illustrate that the models based on the regional data set improved the prediction accuracy. The ensemble classifiers yielded better results than single ones. The re-sampling methods benefit from the improvement in forecasting of a few events, which are the ozone threshold exceedances. The CART-based ensemble algorithms perform better than the SVM and ANN. The results of SMOTE combined with CART based on the distance-based data set were noteworthy. However, the ensemble algorithm Adaboost is strongly recommended based on the statistical comparison of the results in this case. In comparison with the regression mode with a tuning threshold, the classification mode with preprocessing methods performs better for ozone threshold exceedance forecasting. From the results of the variable importance analysis, it can be concluded that the most important factors affecting ozone forecasting are the $\mathrm{O}_{3}$ concentration level at a station's nearby stations, and weather (i.e., wind and temperature) and the description variables - month and season. 


\section{Chapter 6}

\section{Multi-level Awareness of Energy Used in Production \\ Processes(Microcosmic)}

This chapter will present the concept of multi-level energy awareness, and more efforts will be spent on the core of this system - process data analysis. The details of the data sources have been described in Chapter 2, Section2.1.5. The energy data from smart sensors were stored in and managed by NonSQL database-ElasticSearch(Chapter 2 , Section 2-16). Via the interface from $\mathrm{R}$, the data manipulation and data analysis are operated in $\mathrm{R}$ (Chapter 2, Section 2.3.3). This concept of multi-level energy awareness and findings of the pilot study have been published in the Journal of Cleaner Production 232].

\subsection{Concept of multi-level energy awareness}

Based on monitoring levels and factories orientation, energy awareness can be classified in two ways, as shown in Table 6.1. First, energy awareness orientation for machinery aims to increase awareness at the process, machine, production line, and production system levels. The main focus above is to monitor the energy consumption of machines and production lines by linking a particular product ID, order ID 
with energy data. The target of machinery orientation mainly refers to define energy efficiency of a machine or production line. Second, energy awareness orientation for products seeks to increase awareness regarding the operation, product and order levels. In order to achieve this, energy data should be collected in real time at the machine level, and be linked to production data to determine the energy information for specific operation IDs, specific product IDs, and specific order IDs, as explained in the following paragraphs.

Table 6.1: Awareness level based on monitoring the level and factories orientation

\begin{tabular}{|c|c|c|c|}
\hline Awareness type & $\begin{array}{l}\text { Awareness } \\
\text { orientation }\end{array}$ & Awareness level & $\begin{array}{l}\text { Main beneficiaries (level } \\
\text { of benefits) }\end{array}$ \\
\hline Energy used & Machinery & Process level & Production Management \\
\hline Cost of energy used & & Machine level & $\begin{array}{l}\text { Maintenance Manage- } \\
\text { ment }\end{array}$ \\
\hline \multirow[t]{9}{*}{$\mathrm{CO}_{2}$ emissions } & & Production line level & General Management \\
\hline & & Production level & General Management \\
\hline & & & Regulation level \\
\hline & & & Society level \\
\hline & Products & Operation ID & Schedule Management \\
\hline & & Product ID & Customer level \\
\hline & & & Factory level \\
\hline & & Order ID & Factory level \\
\hline & & & Market level \\
\hline
\end{tabular}

The energy awareness orientation for machinery includes the following: Energy awareness at the process level seeks to understand the energy pattern used to complete an operation on one or more machines. Here, the objective is to define the energy used without linking the process to a specified product ID or order ID. Energy awareness at the machine level means that a machines energy data are collected, retrieved, 
and analyzed to define its energy consumption pattern. Energy awareness at the production line seeks to define the energy consumption of a sequence of machines that process one job. Finally, the total energy consumption of several production lines provides energy awareness at the production system level (see Table 6.2).

Table 6.2: Capabilities possible with energy awareness orientation for machinery Machinery Orientation:

Process level

Evaluate specific e-KPIs.

Identify the power usage patterns in the production of parts that are responsible for rising energy costs.

Identify specific process inner variability in terms of energy demand, as well as the likelihood of issues.

Machine level

Identify the energy consumption ratio of the specific production process. Apply this to diagnose the state of the machine for specific operations.

Be further aware of the machine energy consumption and company assistance to note the machine status, helping to determine anomalous machines.

Production line level

Adjust loading parameters to rebalance the full set of factors including energy.

Better accountability based on integrated KPIs .

Production level

Order scheduling for production line, according to integrated performance.

e-KPIs

It is important to emphasize that not only is the energy usage for the specific operations collected here, but also the machinery consumption for inter-product operation (the time between processing the actual product and starting the next one) is gathered. Energy awareness orientation for products that includes specifying energy 
awareness at the operation level looks to specify the energy used for one operation for a specified part or product. This is done by calculating the energy used between the starting and finishing times of the operation.

Determining the energy that is used at the operational level makes it possible to calculate the energy used to produce a product (i.e., energy awareness at the product level). This awareness can be achieved by calculating the total energy used during all operations that are carried out to process a specific product ID. The energy used in logistics can be added to the total energy used. Here, logistics is considered to be one operation of the part/product. So, it contributes time and energy data. When operations are integrated, the energy used for the separate operations can be determined. For example, in moving a bundle of components, the energy used to move them divided by the number of components moved will yield the energy used per component. Finally, energy awareness at the order level can be defined by calculating the total energy used for producing all products in a customers order (see Table 6.3).

\subsection{Target: Energy used, its cost and $\mathrm{CO}_{2}$ emis- sion}

The cost of energy consumption is an important issue for factories. This is mainly because the cost of the energy used by a manufacturing plant commonly represents the second largest operating expense in several industries [233. Although this is not the case for all manufacturing facilities, it is a significant part of the variations occurring per product, because raw materials and human resources tend to be defined on a fixed basis. Therefore, determining the energy used at the operation level (by calculating the energy used between the starting and finishing times for the operation) is useful for factories, because this information can be used to improve energy efficiency.

Because energy prices can vary throughout the day (e.g., on an hourly basis), the cost of the energy used to produce a product can be calculated by aggregating the 
Table 6.3: Capabilities possible with energy awareness orientation for products.

Products Orientation:

Operation level

Identify the energy usage at operation level to produce the products.

Calculate the KPIs that help to integrate information at the product operation level.

Identify the energy cost in monetary value, especially the non-value-added cost in order to identify possible savings.

Product level

Measure the energy usage, energy cost and carbon footprint to provide specific environmental information to customers of products.

Order level

Intrinsic variability of energy usage, cost, and environmental impact can help customers to size their orders when additional information at this level becomes available, as different product strategies are possible depending on the order size.

Enables benchmarking, when the carbon footprint is taken into account.

costs of energy used throughout the entire operation of product production and order production. In some countries, commercial and industrial customers are billed in part by the peak electricity demand observed during a certain period. This demand charge is in addition to the charge for the electricity that they consumed and can apply to as much as $45 \%$ of their electricity bills. Regarding this dimension, transparency in energy peaks induced per operation is certainly relevant for implementing coherent operation sequences throughout the shop floor.

Energy awareness at the operational level enables factories to calculate the amount of $\mathrm{CO}_{2}$ emitted while processing a product or satisfying the total order and to provide adequate and reliable information to their stakeholders. In a general way, energy providers give $\mathrm{CO}_{2}$ data to their customers[234]. At the present, some energy providers advertise $100 \%$ renewable energy 235$]$.

In reality, manufacturing companies can buy energy from several energy providers. 
This range of choices allows their energy purchasing decisions to be made after considering energy sources and prices, and whether the sources are renewable or not. Furthermore, some factories generate a part of their energy from renewable sources on-site and buy the rest. In such cases, the factories can calculate the $\mathrm{CO}_{2}$ emissions (e.g., per product) based not only on the amount of energy used in producing the products, but also on the energy sources used to generate that energy. This enables factories to show their stockholders how much of the production processes of a product can be considered to be green and to what end. Table 2.1.5 provides equations to illustrate how to calculate the energy used, energy costs and $\mathrm{CO}_{2}$ emitted per operation, per product and per order

\subsection{Requirements to enable multi-level energy aware- ness}

In order to implement the multi-level energy awareness, three aspects are relevant and needed to standardization, including energy data, production data, and techniques for realization for building system. For the techniques part, it is consisted of data storage, data computing, and result visualization tools.

\subsubsection{Energy data}

Via the installed smart sensors on machines and equipment, energy data with two or three seconds interval time can be selected in real time. Under this situation, energy consumption data with specific starting time and finishing time can be extracted precisely. Figure 6-1 presents an example that depicts the energy consumption for a machine during production process. In this figure, it shows repetition trends from a

period of time. Each of the repetition correspond a operation along with the starting time and finishing time. 


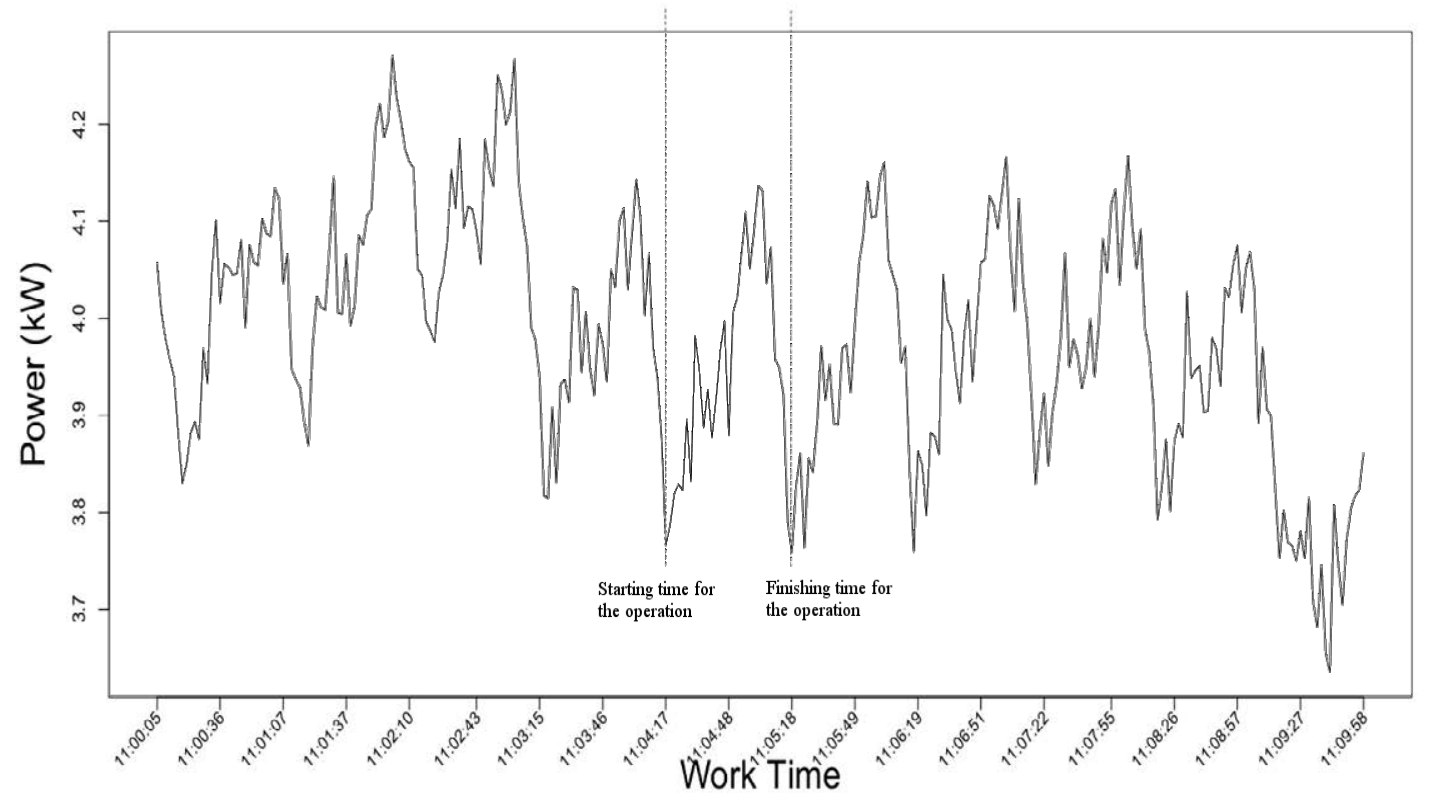

Figure 6-1: Energy consumption by a machine for the production processes

\subsubsection{Production data}

Providing the energy usage for each process and product, the production data are required for linking to energy data. Therefore, production data with several components are expected to be obtained for factories.

- The details of technical specifications of all the process must be provided properly from the factory, which should be coded and stored in a digital form, and read for machine-to-machine(M2M) queries.

- The mapping between each production resource(machine,equipment) and processes must be available.

- The parameters configuration must be valid and established. It should includes the description of performance and the expected quality levels. The set of these parameters will be related to the production process and production resources.

- Digitally establishing the production plans per order and/or production via the manufacturing systems, such as Manufacturing Execution System, Enterprise 
Resources Planning, Manufacturing Resources Planning etc. This plan must be available based on the request for both human consumers and M2M dialogs.

- The records with all the events occurring during the activities of the production on a device should be provided by a log system. The details of the records includes the product ID being processed at any resources with the starting and finishing times for each process, and the product ID for the specific process and operation.

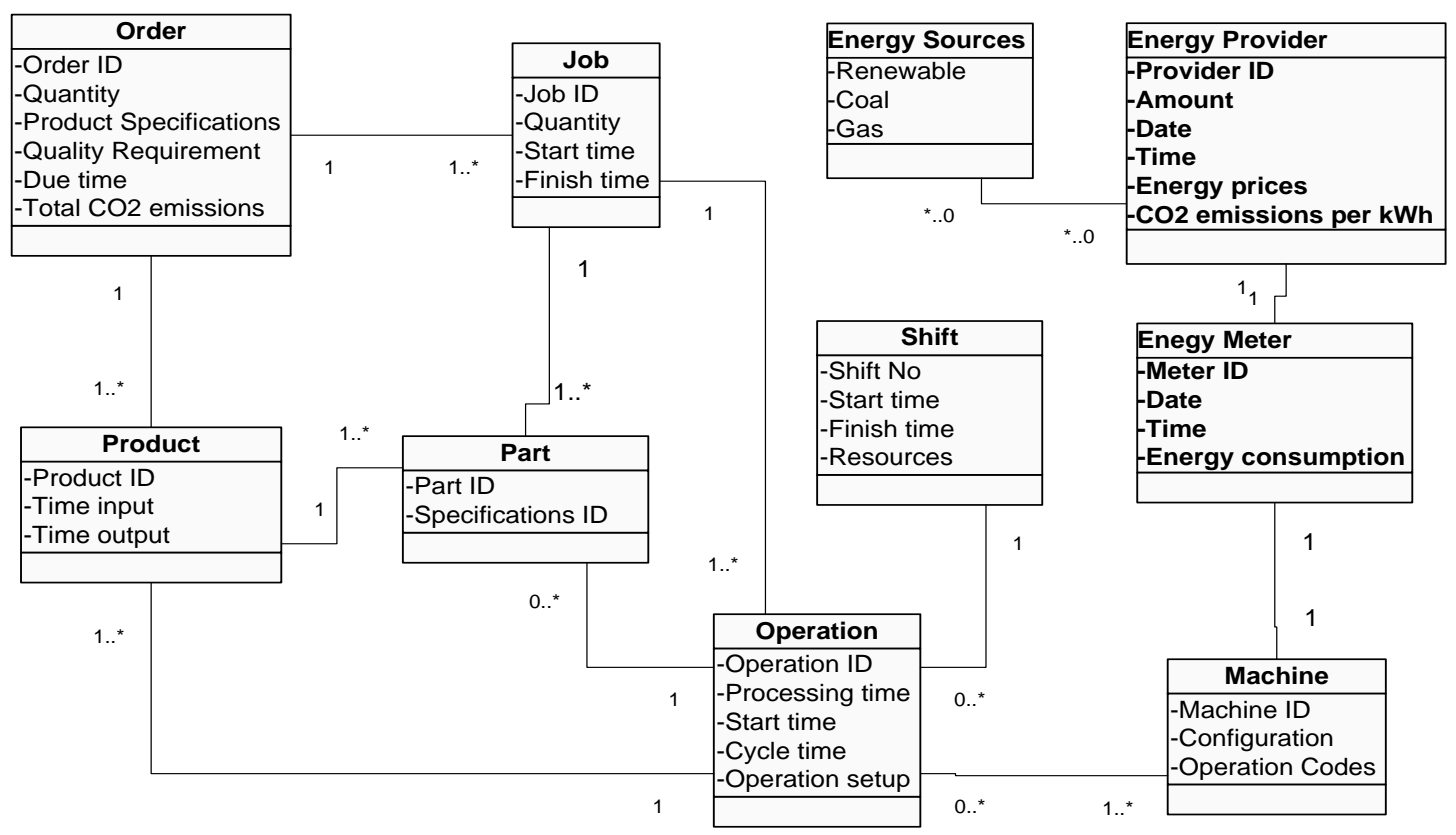

Figure 6-2: Class diagram for energy-aware production management

Figure 6-2 depicts a class diagram (i.e., data entities) aiming to show the main structure of energy-aware production management. It includes three sets with ten classes. The first set includes order, part, and product classes, which represent information about orders and products. The second set includes job, shift, process, and machine classes, which represent information about scheduling and operations logs. The third set includes energy meters, energy provider, and energy sources, which represent data about energy information that is used for processing. 


\subsubsection{Technique requirements}

For maximum utility, the above-described data must be available to those persons at the company who have authorised access and to the information processing units of the company.

Data gathered from smart metering devices and from machine controllers are uploaded into convenient data storage systems. As many different data streams will be uploaded, specific big data architecture should be established. Therefore, the lambda architecture, proposed by [236], is recommended for this purpose, as it is based on several assumptions (i.e., fault tolerance, support of ad-hoc queries, scalability, and extensibility), and is composed of three layers. The First Batch Layer is responsible for managing the master dataset and for precomputation of batch views. It can be implemented by the use of systems such as the previously mentioned MongoDB or Hadoop. Furthermore, the Map-Reduce algorithms contiguously compute views of those data that are available to the various applications. The Second Serving Layer indexes the batch views for fast ad-hoc queries. Finally, the Speed Layer serves only the new data, which have not been processed yet by the Batch Layer. The role of the Speed Layer is to compute in real time the data that have just arrived and have not yet been processed by the Batch Layer.

Based on these data layers, specific information is created by different agents, structured according to the related level of information being produced. For example, an agent would process end event messages by resource type and then look for the related starting event. Once the time window is estimated, the effective energy can be calculated, aggregating the results and preparing fixed features in specific views for further analyses. Integration between operation-related energy data can be achieved according to the followed scheduling, making comparisons between different strategies or configurations regarding time, energy, costs, quality, etc. at VSM aggregated level.

Agents are autonomous and distributed. Cooperation between agents is achieved using message passing, where all agents communicate in a similar manner. Therefore, the integration is simplified. An agent is implemented to calculate energy data per 
operation ID. The agent first calculates the energy data for each operation applied to the product, and then sums the relevant values. A second agent can be implemented to track the sequence of scheduling and select information per product ID. This is intended to clarify the energy expended per product. The information is stored at the upper level in the class model. A third agent is needed to look into the summary records database, the process log, and the process definition database in such a way that processes parameters based on data can be analyzed by the process log and summary records. Therefore, events can be analyzed and rules to improve the knowledge management can be derived. Several other agents can be defined by specific purposes to look deeply into different relationships regarding quality, operational strategies, etc.

\subsection{Pilot study}

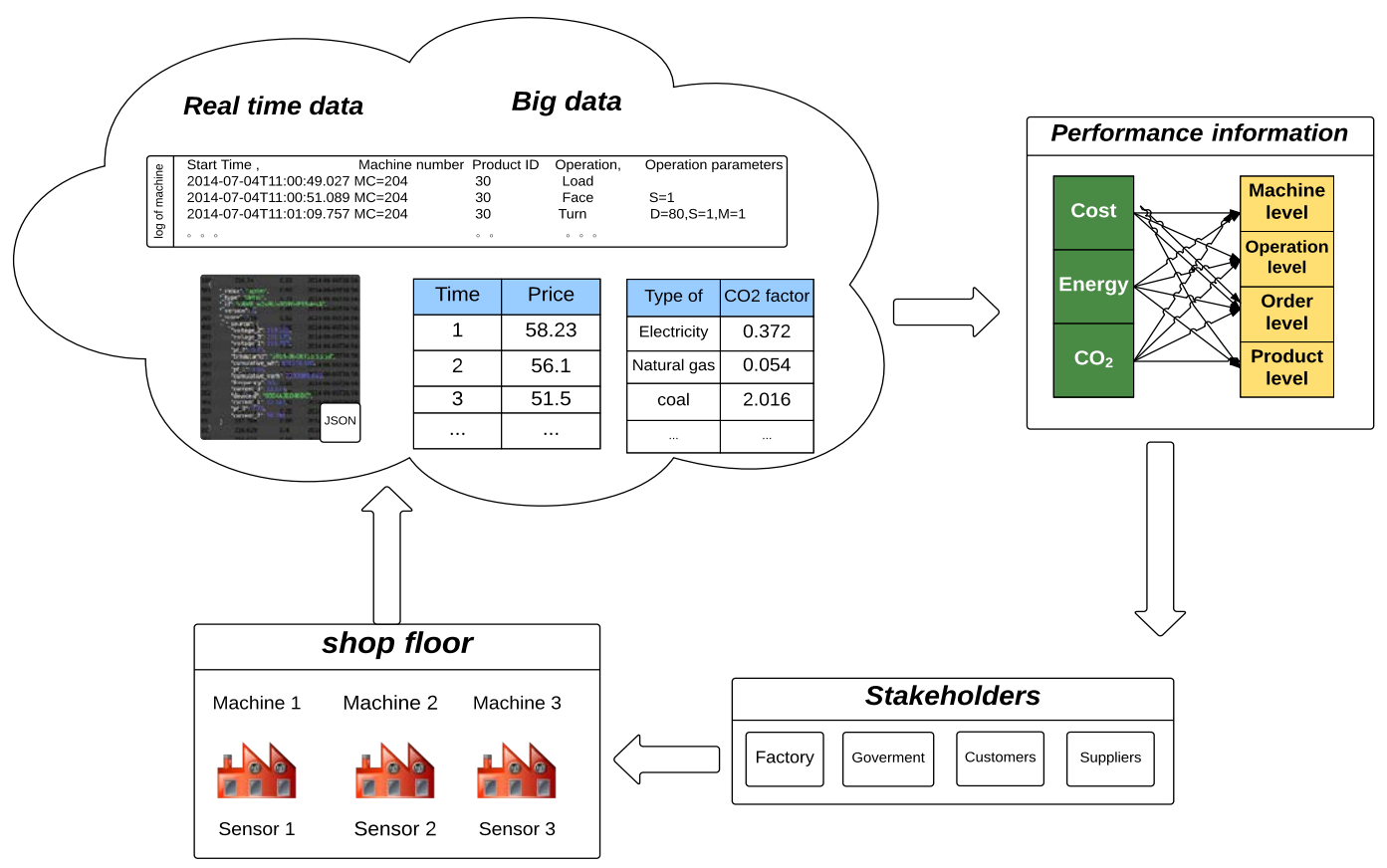

Figure 6-3: Framework 


\subsubsection{Energy awareness at operation level}

The first software agent was used to total the energy consumption, cost of energy used and $\mathrm{CO}_{2}$ emission of all products of a particular operation in order to analyze the operations consumption behaviors. Figure 6-4 shows the cost of energy per operation for the 172 products involved in this part of order number N1529. The operations Turn and Face are the most energy-consuming and have the highest energy cost. They are followed by the operations Chamfer and Thread. Operations Load, Remove and Unload consume relatively less energy and cost less than the other operations. The $\mathrm{CO}_{2}$ emissions by operation are summarised in Table 6.4.
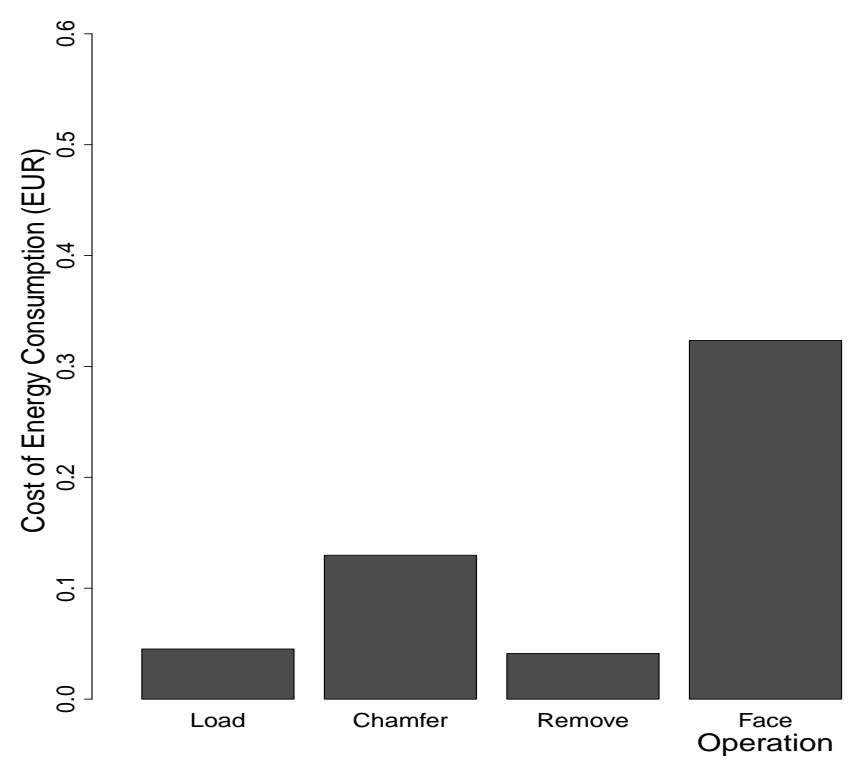

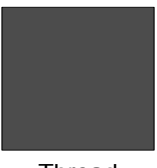

Thread

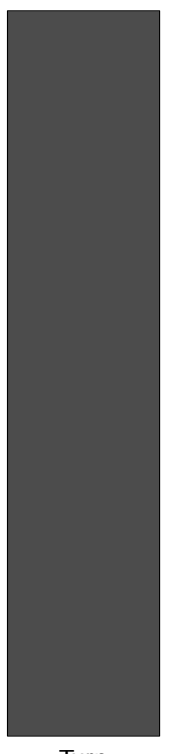

Turn

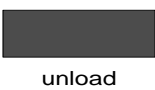

Figure 6-4: Cost of the energy used by operation (operation level)

Table 6.4: CO emission by operation (operation level)

\begin{tabular}{|c|c|c|c|c|c|c|c|}
\hline Operation & Load & Chamfer & Remove & Face & Thread & Turn & Unload \\
\hline $\mathrm{CO}_{2}(\mathrm{~g})$ & 139.5 & 400.4 & 126.8 & 998.3 & 397.6 & 2018.8 & 130.5 \\
\hline
\end{tabular}




\subsubsection{Energy awareness at product level}

The second software agent was employed in order to increase the awareness of energy at the product level. This agent can obtain the energy consumption, cost of energy used and $\mathrm{CO}_{2}$ emission per product. For example, Figure 6-5 shows $\mathrm{CO}_{2}$ emissions for the first 30 products in the part of the order N1529 related to the A-118951 item (see Table 2.7). It clearly indicates that product ID 14 and product ID 15 used much more energy and produced more $\mathrm{CO}_{2}$ than the other products.

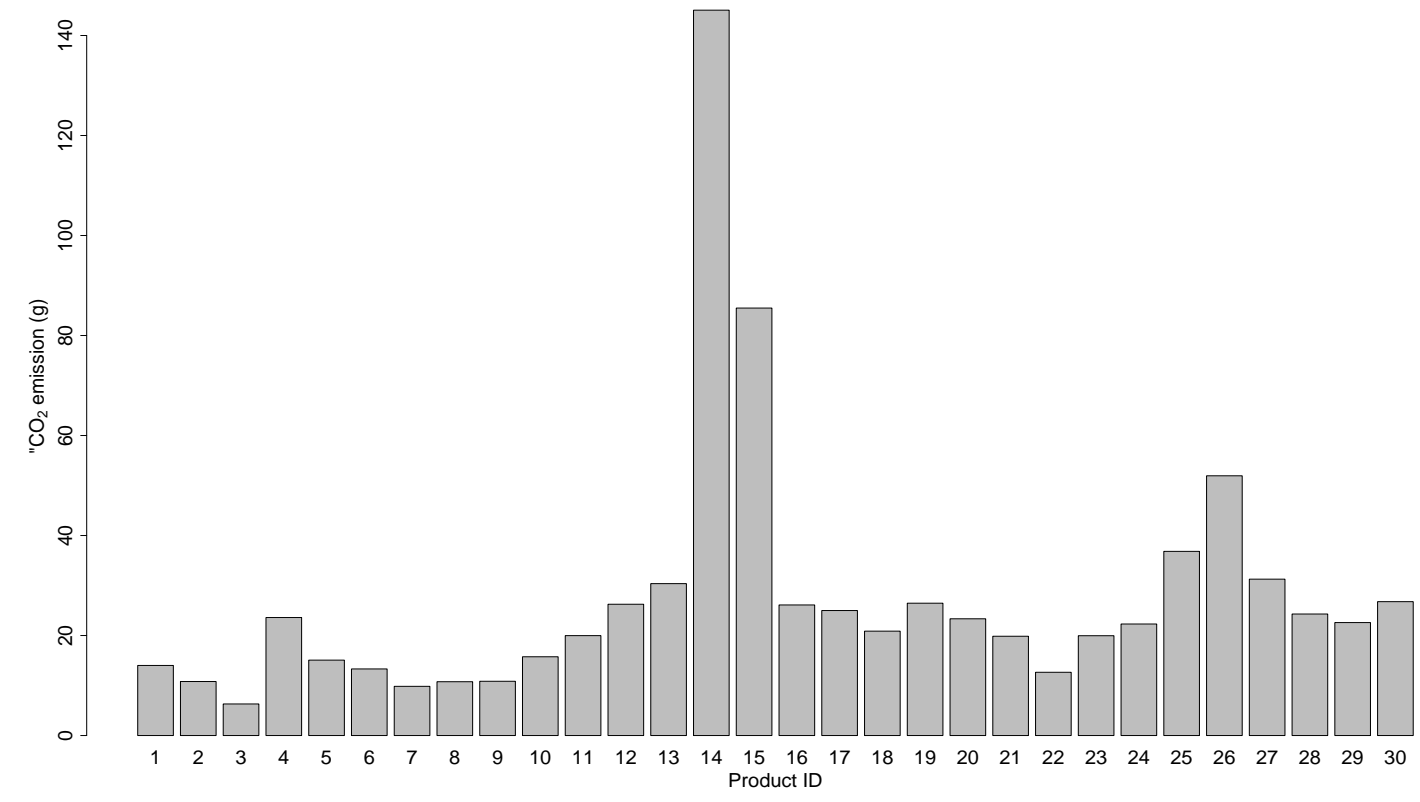

Figure 6-5: CO emission by product (product level)

To understand why product IDs 14 and 15 consume so much more energy than other products in the same family, the energy consumption records of product IDs 13, 14, 15 and 16 were extracted and are presented in Figure 6-6. From 10:42:21 to 10:43:31, the machine actively produced product ID 13 . The next three periods were used to produce IDs 14, 15 and 16 respectively. There may be several reasons for the high energy consumption of product IDs 14 and 15. First, high power was required by the machine, which can typically be explained by mechanical problems. Second, it took a much longer time to produce these two items than the others. A third scenario could be a combination of the two foregoing problems, namely that 
there was a mechanical breakdown and the two items took longer to produce than the others.

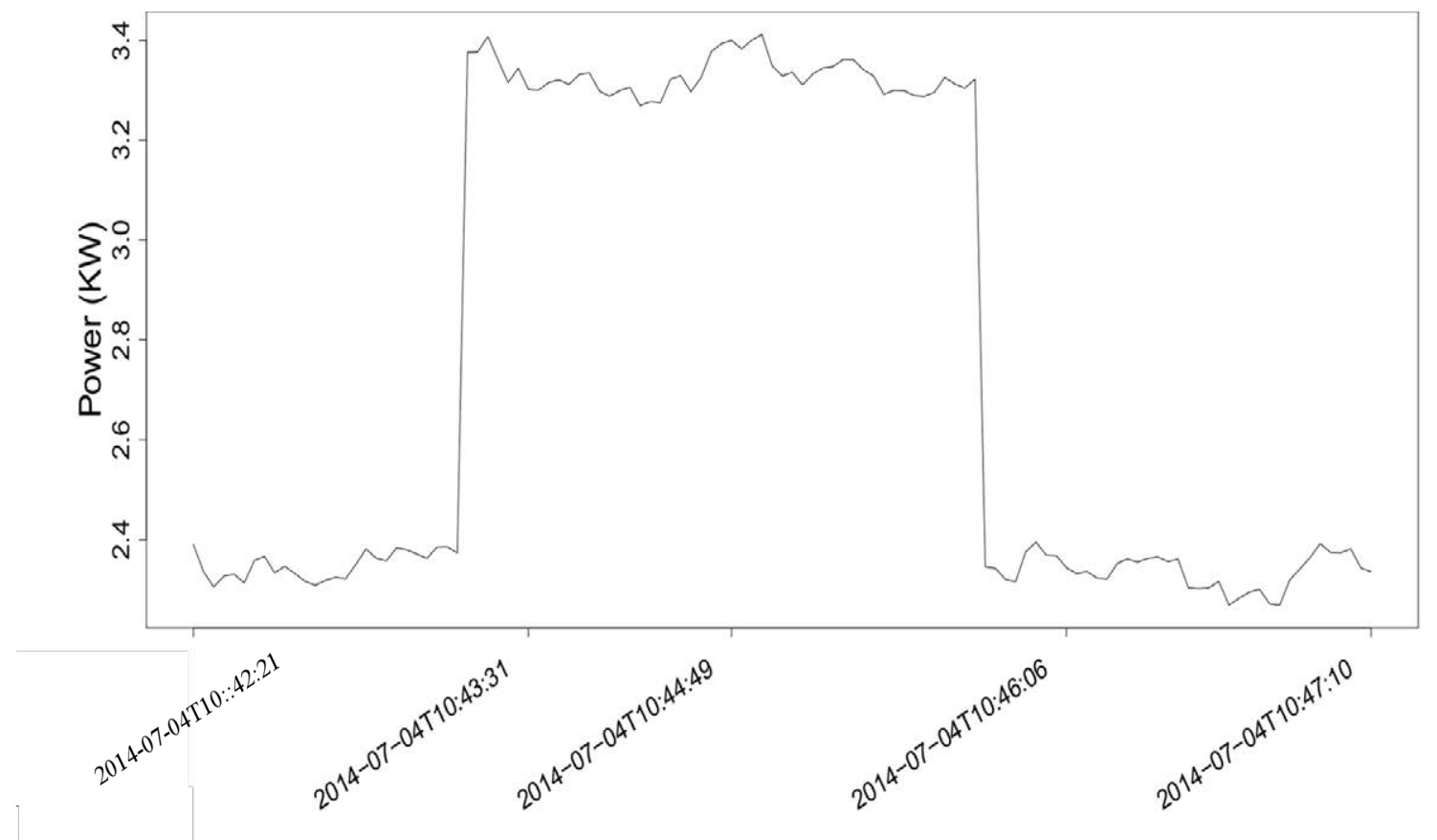

Figure 6-6: Energy consumption of product IDs 13, 14, 15 and 16

According to Figure 6-5, it can be concluded that the high energy consumption levels for IDs 14 and 15 are due to the first proposed reason, as the duration of their production looks not very dissimilar to the other items. The mechanical properties of these two items are quite different from the others. This particular effect shows the additional uses of smart production to exploit benefits in the whole value chain, as it helps to identify potential and unwanted dissimilarities that could affect customer satisfaction.

\subsubsection{Energy awareness at order level}

Finally, the awareness of the energy used in the higher order levels can be achieved by totalling the energy consumption, energy used cost and $\mathrm{CO}_{2}$ emissions of all the products and all operations in the same order as followed in Table 14.

The variations in energy consumption of products from order 1568 are shown in the Figure 6-7 sustainable value stream mapping. A more aggregated value at order 
level appears in Figure 6-8, which shows the amount of energy that was wasted (i.e., the amount that was in excess of what was used directly for production).

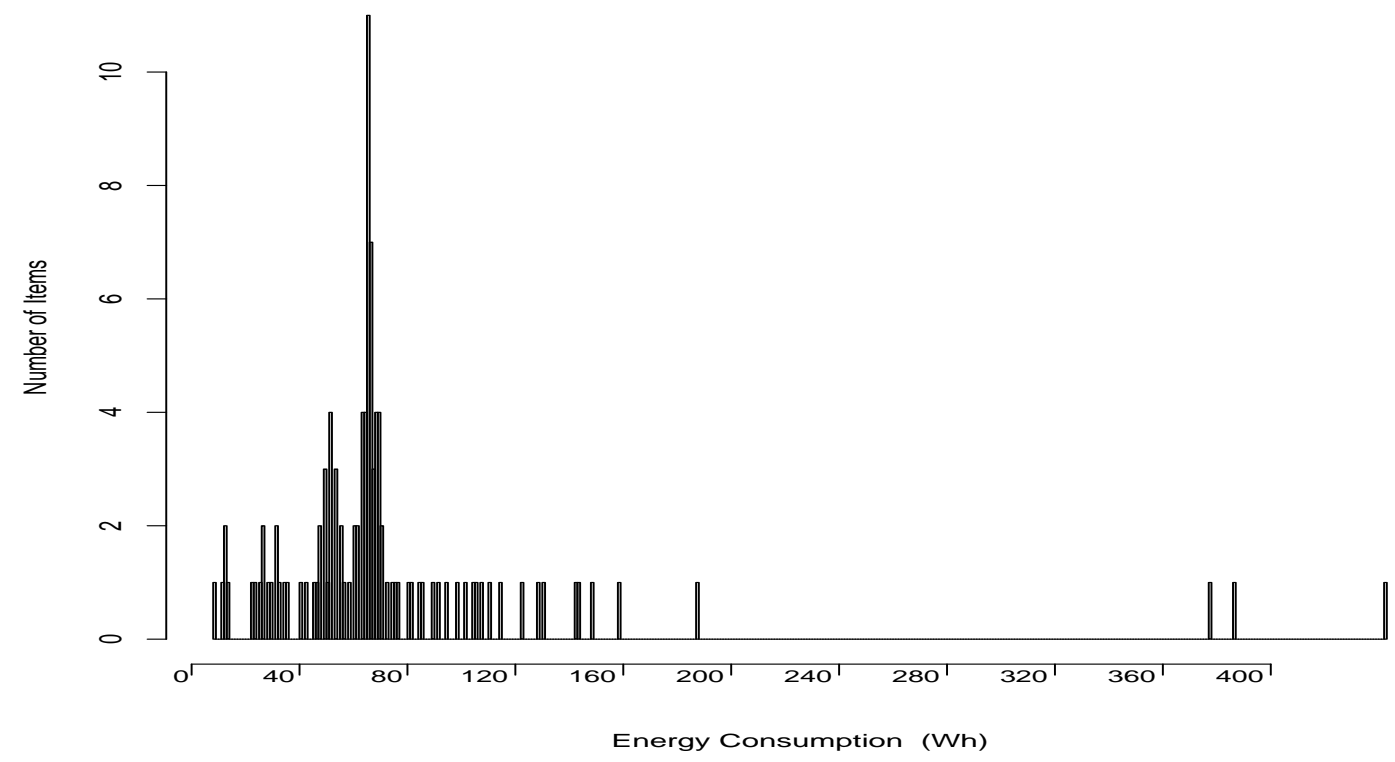

Figure 6-7: Energy distribution of products from order N1568.

\subsection{Discussion}

Thanks to smart metering with big data tools and M2M communications, energy usage data can be collected and tracked at operation and product levels. The present paper introduces a specific and detailed method to integrate real-time energy usage data at the operation level with production and scheduling data. In fact, it makes it possible to specify the energy used, energy costs, and the amount of $\mathrm{CO}_{2}$ emitted for production in a single operation, single product, and single order. It also enables factories to specify the $\mathrm{CO}_{2}$ emitted in producing a product based on the energy sources that were used in production. All these aspects increase transparency at component level and show to what extent energy awareness is increased.

Finally, this study enables the use of e-KPIs to identify their environmental impact, as estimating energy wastage becomes simpler. Additionally, a proposal for 


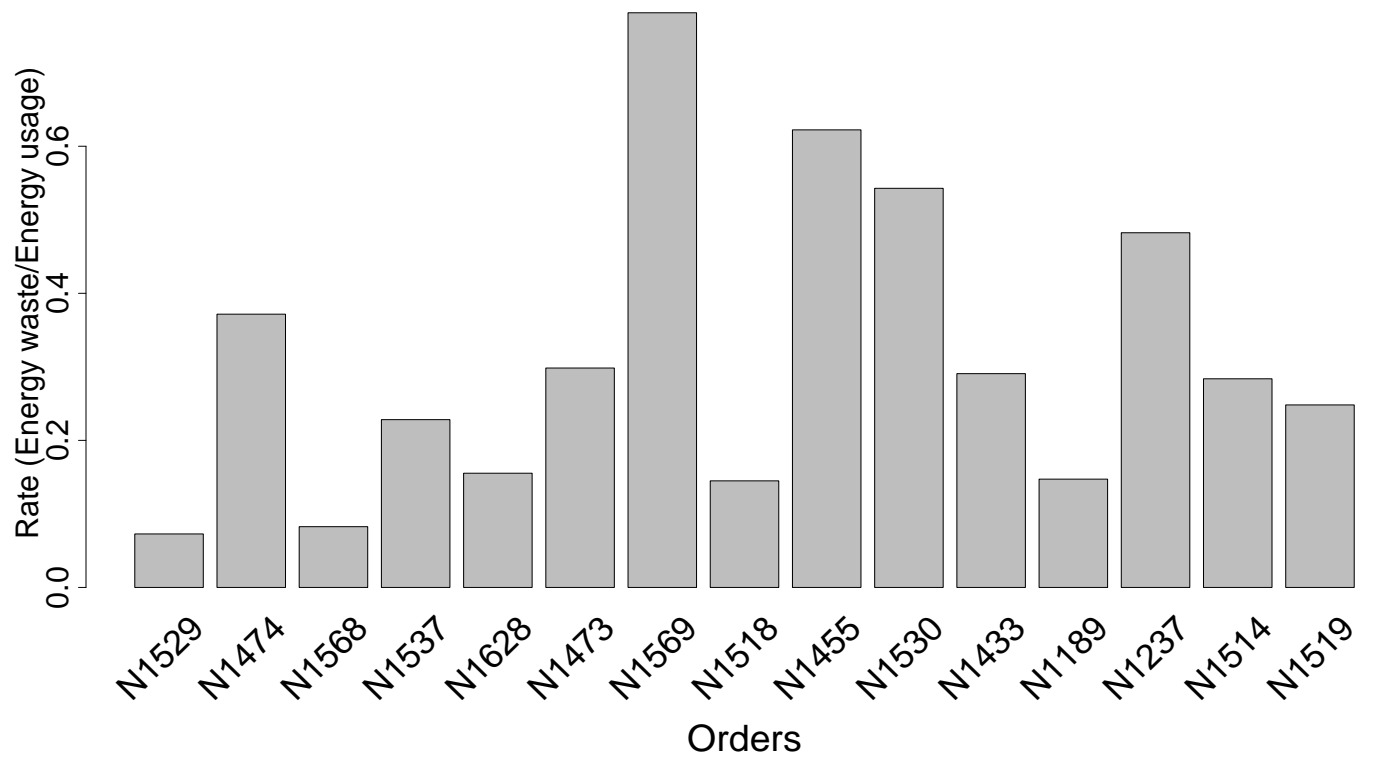

Figure 6-8: Energy distribution at order level, derived from the case study.

accountability was established in order to enable the integration of several different providers in the value chain. Such a proposal enables different levels of granularity to accommodate different legal agreements regarding privacy and data ownership.

When factories begin to label their products with the level of $\mathrm{CO}_{2}$ emitted during production and the level of energy required to use the product, studies will be able to determine the impact of adding such a label on the purchasing decisions of consumers. Another future study could examine the impact of forcing factories to increase their energy efficiency and use renewable energy sources in production processes to show how environmentally friendly their products are. 


\section{Chapter 7}

\section{Driven Forces of Energy Consumption, GDP and $\mathrm{CO}_{2}$ Emission in China}

This chapter aims to discover the characteristic patterns of energy consumption, GDP, and $\mathrm{CO}_{2}$ in China and their driven forces by using dynamic indicators and cluster algorithms in order to help formulate policy at provincial-level. Agglomerative Hierarchical Clustering(AHC) and Gaussian Mixture Mode(GMM) are employed, which are introduced in Chapter 2, Section 2.2.8 and 2.2.8 respectively. The data source and the calculation of energy consumption and $\mathrm{CO}_{2}$ emissions were described in Chapter 2. Section 2.2.9 and Section2.2.9

\subsection{Description analysis}

Figure 7-1 shows the 15 provinces with highest $\mathrm{CO}_{2}$ emission volumes at the year 1996, 2001, 2006 and 2011. In 1996, $\mathrm{CO}_{2}$ emission volumes of all the provinces were below 25000 thousand tons. HeB, JS, LN and SD are the provinces with the highest $\mathrm{CO}_{2}$ emissions. From 1996 to 2001, the raise of $\mathrm{CO}_{2}$ emission was trivial. Some provinces, like $\mathrm{HN}$, JL and $\mathrm{SC}$, showed a downtrend in $\mathrm{CO}_{2}$ emission. The most notably increasing of $\mathrm{CO}_{2}$ emission began since 2001. $\mathrm{CO}_{2}$ emission boost in some 
areas such as HeB, HuN, IM, JS and SD. In SD province, it increased twice to 61250 thousand tons in 2011, compared to 25000 thousand tons in 2006. In 2011 IM province suffered the severest $\mathrm{CO}_{2}$ pollution with more than 12500 thousand tons, followed by SD and HeB, with around 7500 thousand tones.

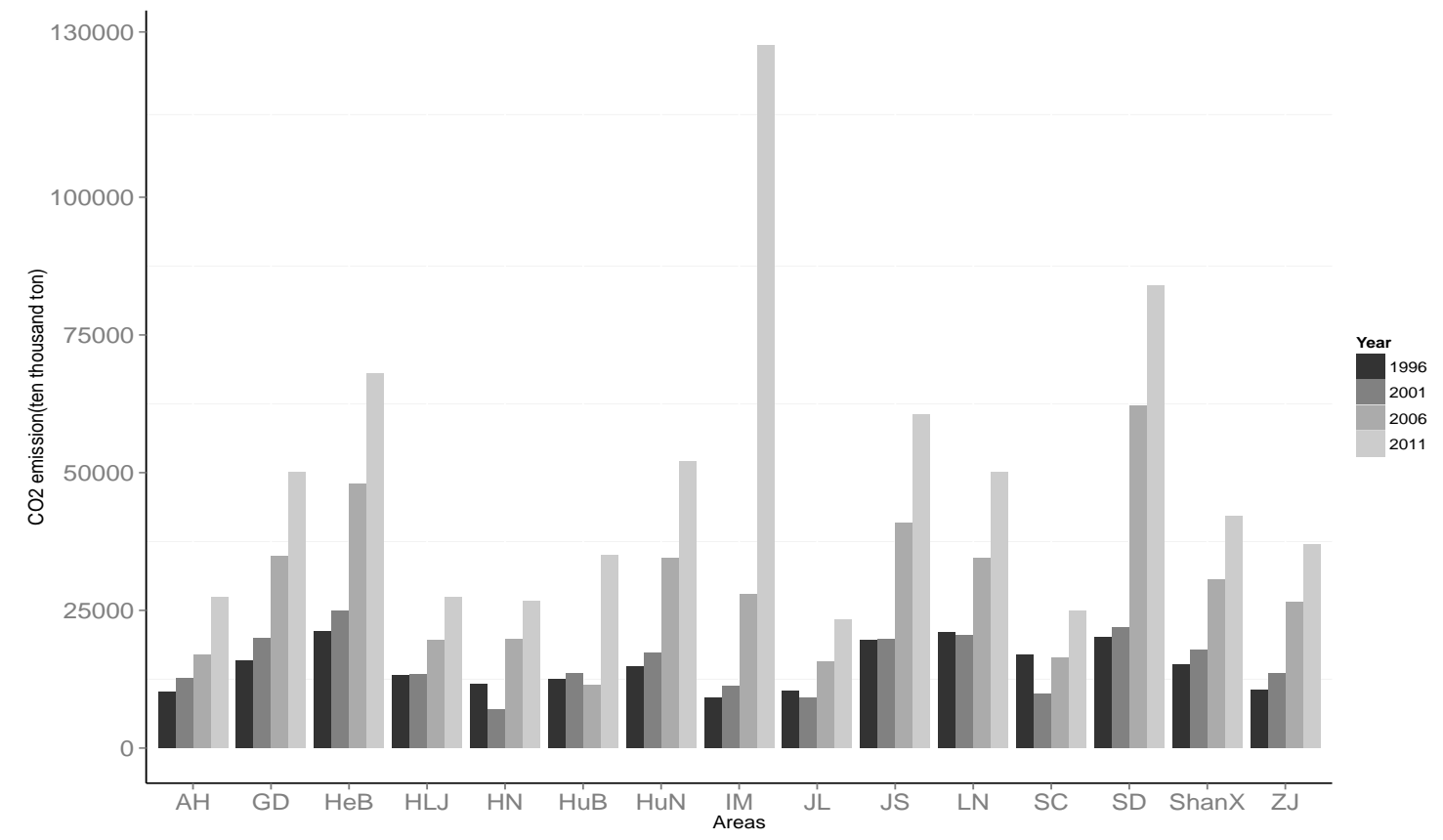

Figure 7-1: The 15 highest $\mathrm{CO}_{2}$ emission provinces in China at the year 1996, 2001, 2006 and 2011.

Figure 7-2 illustrates the GDP values corresponding to the top $\mathrm{CO}_{2}$ level provinces in China, which are GD, HeB, HuN, IM, JS, LN, SD, and ZJ. GD is the area that increased its GDP values notably during this period, from 800 billion in 1996 to more than 5000 billion in 2011. IM grows its economy more slowly than other seven areas, even with highest $\mathrm{CO}_{2}$ emission. It can be seen that beforethe year 2002, the economy of these provinces grow slowly, but considerably rise afterward. Both Figure 7-1 and Figure $7-2$ show the fact that the most pollutant province might not be the most developed one in China (for example IM). Meanwhile, the province, like ZJ, could have higher GDP, but with a relative low $\mathrm{CO}_{2}$ pollution.

Figure 7-3 shows the energy consumption structure in year 2012 in China. The raw coal (66\%) was still the main energy sources in China until 2012. It followed by cleaned 


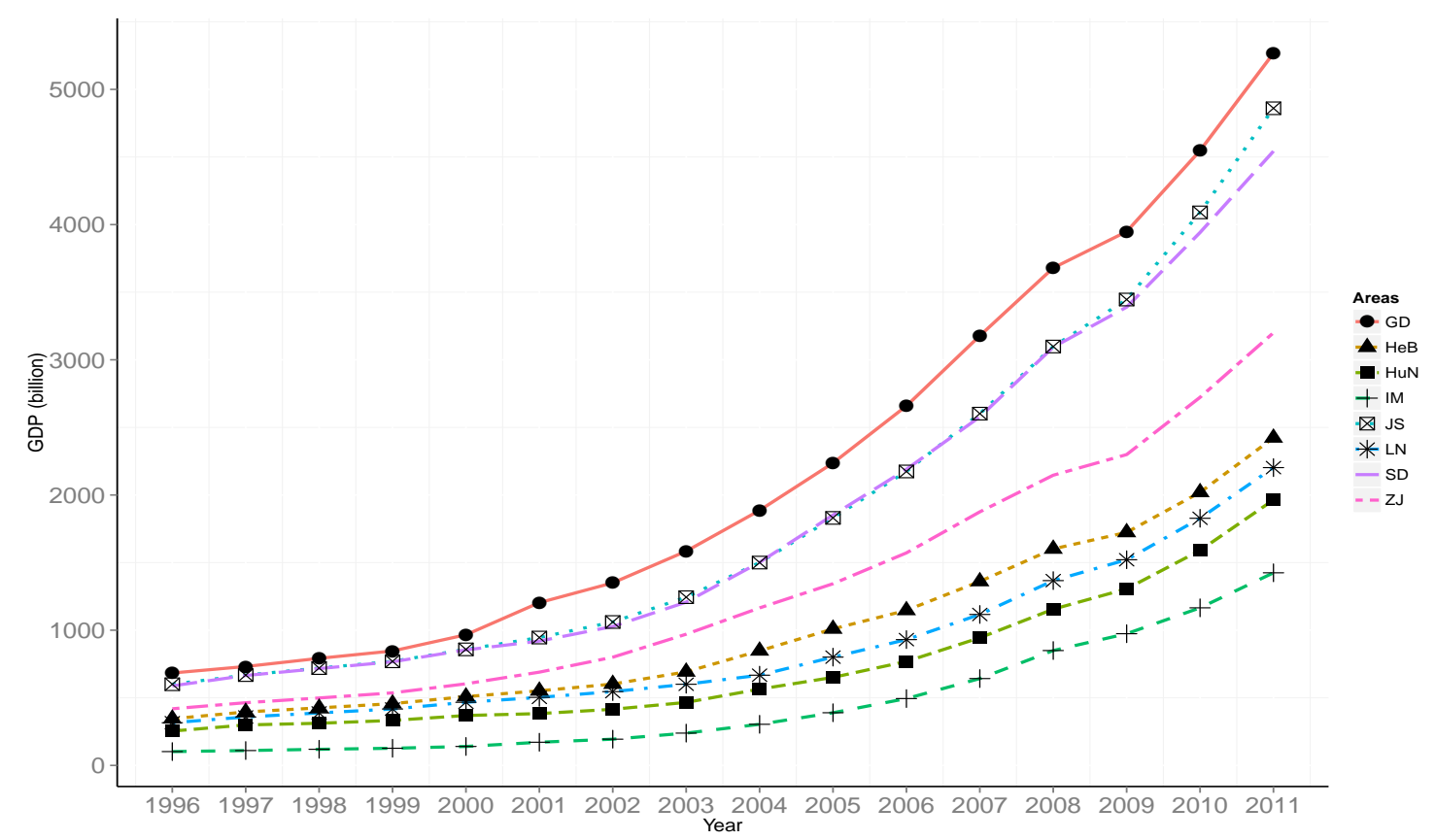

Figure 7-2: GDP corresponding to the selected most $\mathrm{CO}_{2}$ pollutant provinces in China from the year 1996 to 2011.

coal and crude oil, which accounted for $9 \%$ and $7 \%$ of total energy consumption respectively. It clearly demonstrates that the clean energy was not widely used until 2012 .

\subsection{Cluster analysis}

\section{Immaterialisation}

This part will analyze cluster results based on the three indicators: immaterialisation, dematerialization, and decarbonisation in order to discover the similar or different patterns among these thirty provinces. In China, the central government usually makes the major policies and the local government or cities governments are responsible for implementing them. Therefore, it is of importance to understand the characteristics of provinces in the same cluster based on the different indicator in order to design effective policies aiming to reduce emission.

According to BIC (Figure 7-4), the best model based cluster is in model EEV 


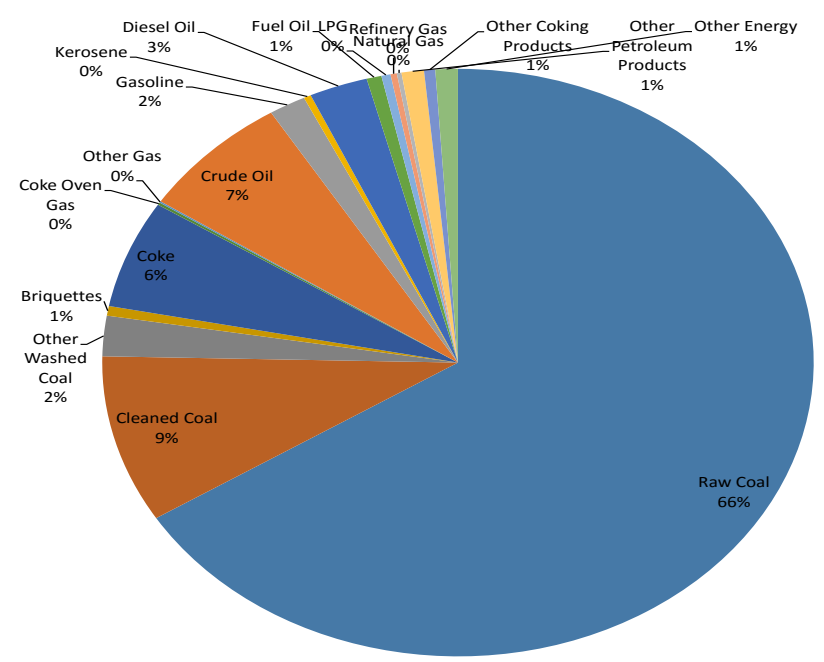

Figure 7-3: Chinas energy consumption structure in 2012.

with five components (clusters) for the changes of $\Delta$ (energy/GDP) in the three FYP. This means the component covariance matrices of this best model are of equal volume but different shape and different orientation. Figure $7-5$ demonstrates the AHC and GMM cluster results based on indicator immaterialisation $(\Delta($ energy/GDP $))$. Based on GMM, the provinces JX and CQ are grouped in the cluster one. LN, JL, HN, SC, ShannX, GS, and XJ belong to cluster two. Cluster three includes ShanX and GZ, whereas cluster four only contains IM. The rest of the provinces are clustered together.

The discrimination patterns of the energy intensity (imaterialisation) among clusters are most probably because of the industrial structures changes from low energy intensive industry to high ones or reverse. One of the measurements that discriminate the industrial structures is the investment in each type of industry, as well as the usage patterns of various types of energy [90].

Figure 7-6 indicates the bar plots of immaterialisation indicator in these three periods. The provinces of the axis are ranked by group according to results of the two cluster methods. This will help to see the pattern of $\Delta$ (energy/GDP) within the 


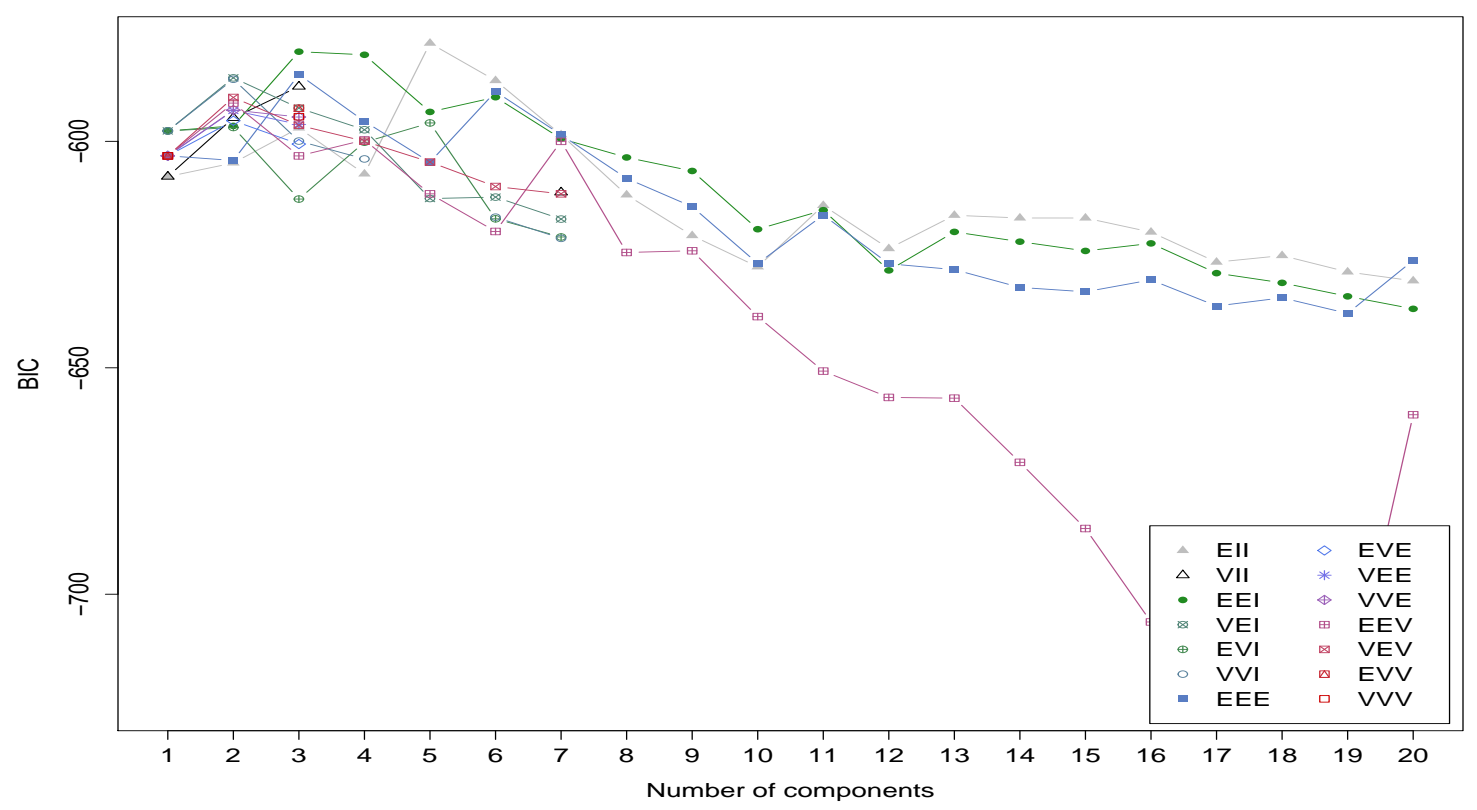

Figure 7-4: 6 BIC for differences of Energy/GDP

same group clearly. The change of energy against of GPD could reflect both material production and consumption from economic production[90]. It illustrates that the energy intensity $\Delta$ (energy/GDP) decreased during the three periods for the most provinces, except the 9th FYP for CQ and JX, the 10th FYP for HN, FJ, SD and YN, 11TH FYP for IM and HB.

As mentioned before, no matter which clustering methods are used in this study, CQ and JX are grouped together. The pattern in this cluster is characterized a considerable increasing energy intensity in the first period and a decreasing trend in the second period, slight changes in the third period. This is probably due to the energy consumption structure of these two provinces. Figure $7-13$ shows the variations in the raw coal percentage of total energy in the three periods corresponding to different provinces. It clearly demonstrates the raw coal as energy consumes accounting to the total energy grows significantly from the year 1996 to 2001. The raw coal percentage still arises since 2001. However, the degree of these shows a notably downtrend afterward. During the 11th FYP (2011-2006), the raw coal percentage decreased or did not change. Meanwhile, cluster GZ and ShanX, there is the same pattern of change 

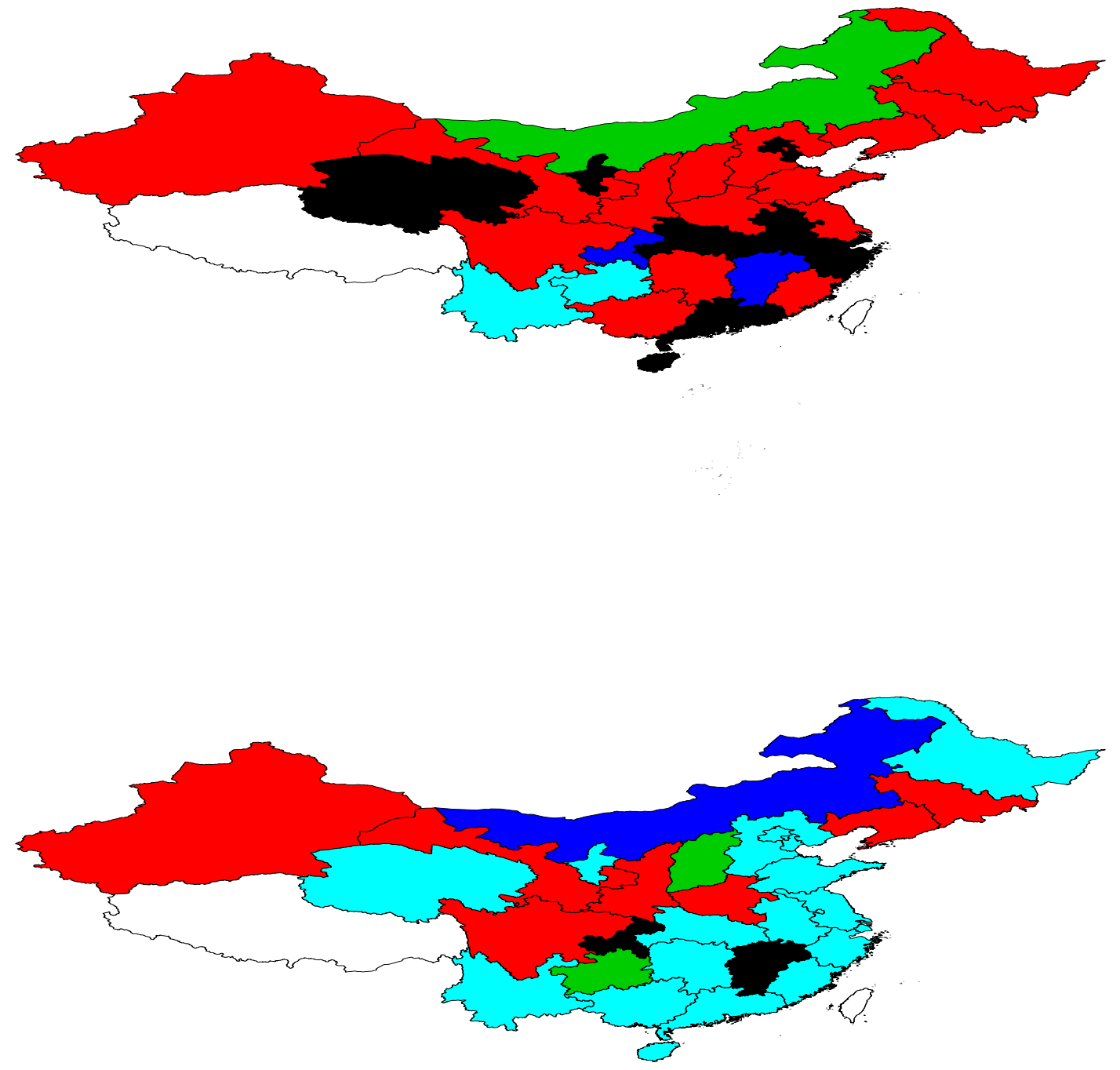

Figure 7-5: AHC results GMM results for immaterialisation indicators 

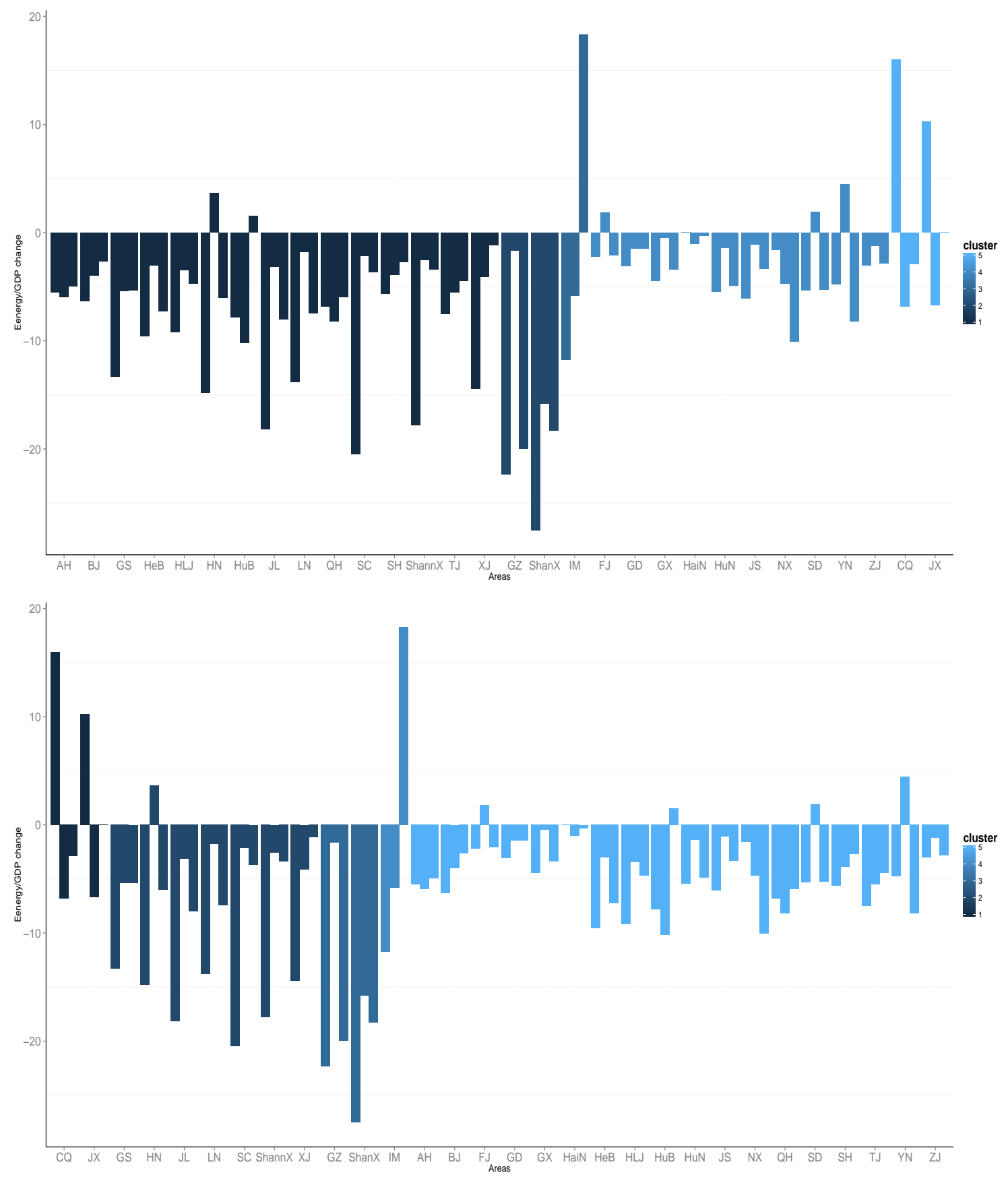

Figure 7-6: Indicator immaterialisation at provincial-level correspond to three periods based on AHC and GMM respectively 


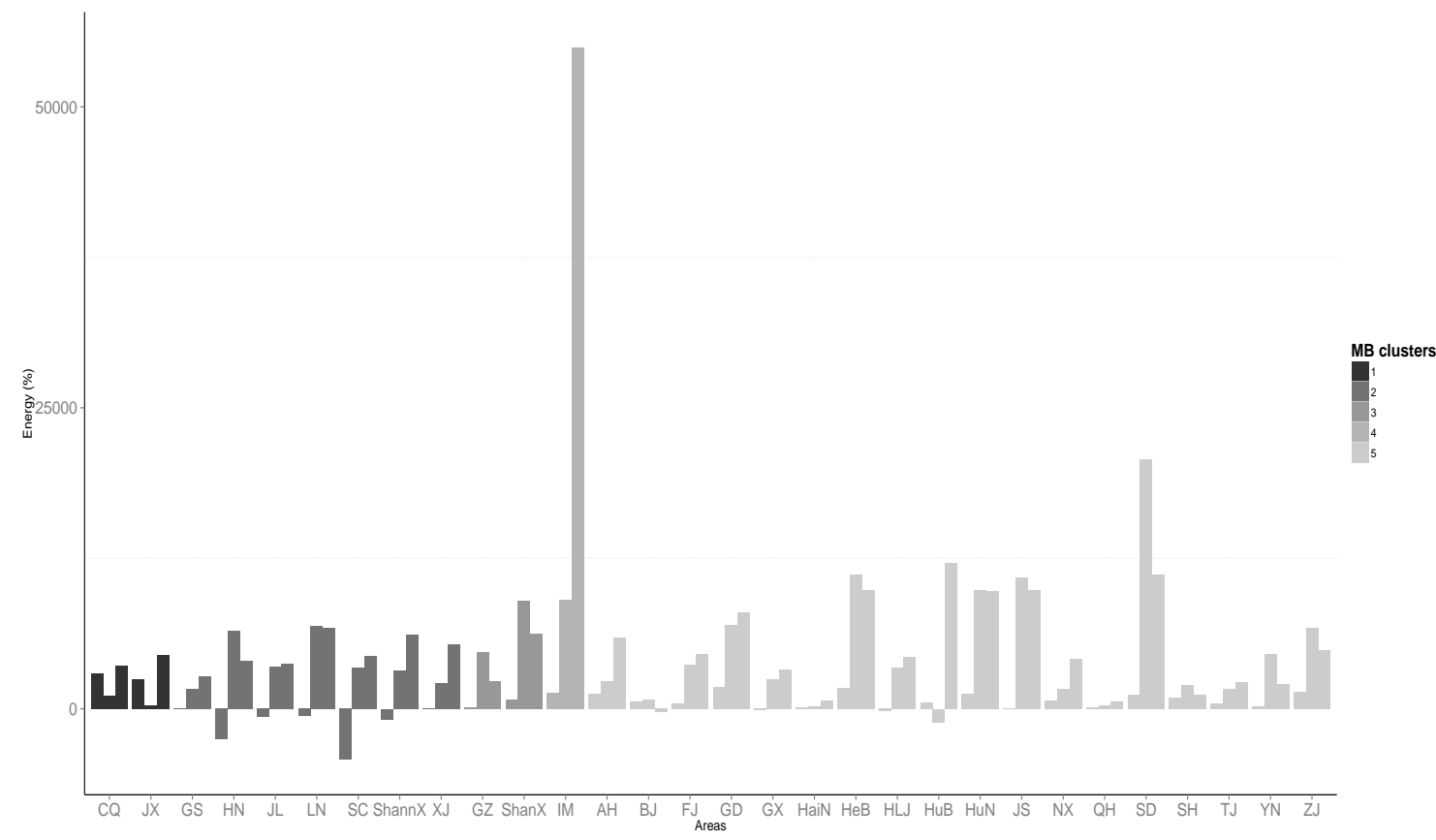

Figure 7-7: Energy changes corresponding to the provinces.

in the energy consumption structure as the change in the indicator immaterialisation.

IM is only province was grouped into a cluster. The pattern for this province is totally different from others, as the energy intensity decreased in the first period, however, arise considerably in the third period. The reason for the pattern is probably due to the change of energy consumption structure (Figure $7-13$ ), but because of the significant increasing of total energy consumption quantity and relative weak arising in GDP in IM province (Figure 7-7 and Figure 7-8).

\section{Dematerialization}

Indicator dematerialization $\Delta\left(\mathrm{CO}_{2} /\right.$ energy $)$ can reflect the environmental harm from material production. Based on this indicator, the provinces are separated into three groups according to BIC. CQ and JX belong to one cluster, while NX and ShannX are grouped together. The rest provinces are in the same cluster. From Figure 7-9, there are considerably changes in provinces CQ JX, NX and ShannX CQ and JX decreased their carbon intensity of energy production $\Delta\left(\mathrm{CO}_{2} /\right.$ energy $)$ significantly. From Figure 7-13, it can be seen that the percentage of raw coal consumption in- 


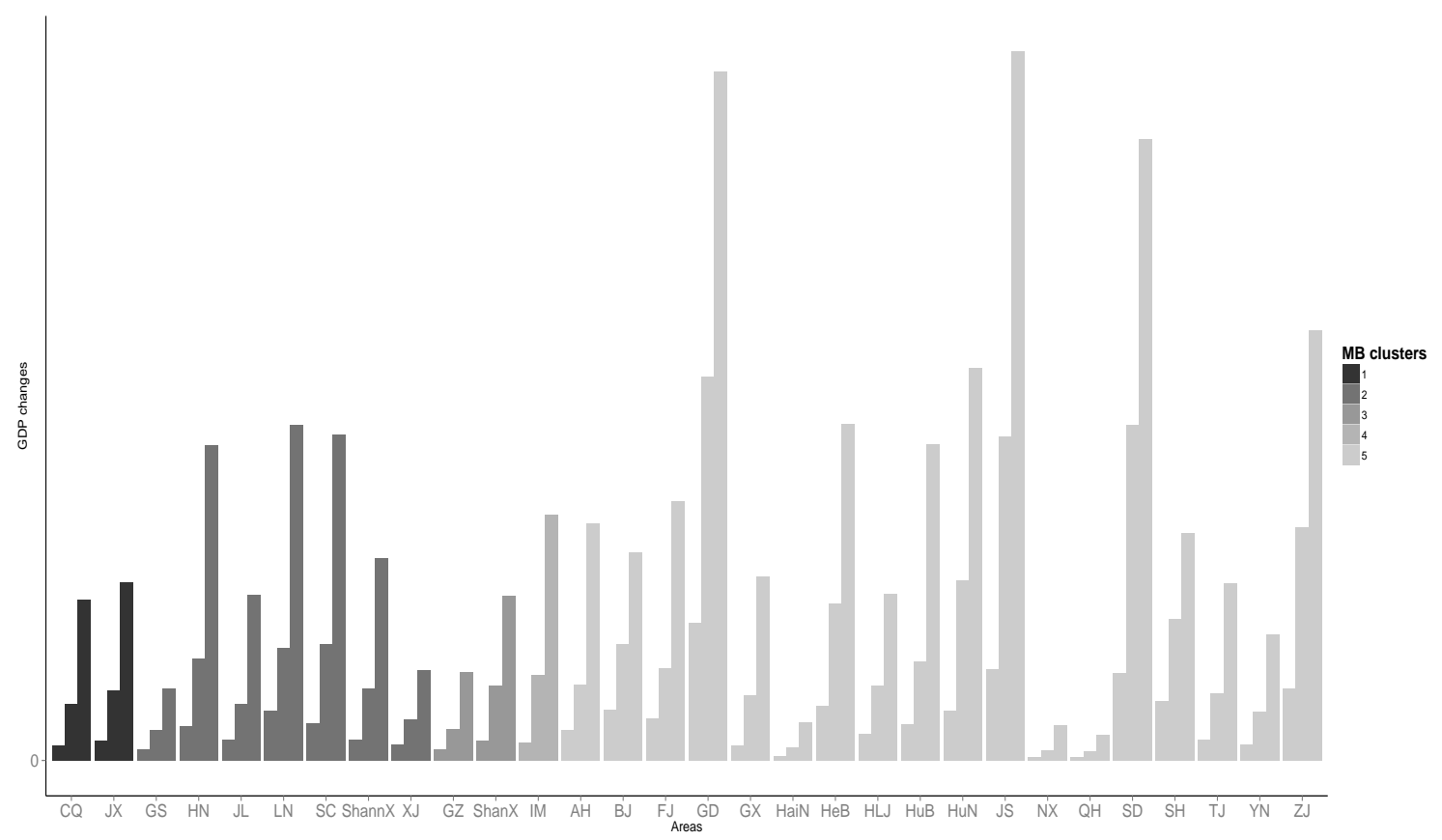

Figure 7-8: GDP changes corresponding to the provinces.

creased considerably during the 9th FYP, rise trivially in the 10th FYP, but showed down trend in the 11th FYP. The clean coal consumption decreased in the 9th FYP. According to IPCC, $\mathrm{CO}_{2}$ emission of clean coal is higher than the one of raw coal. Therefore, the notable increasing of $\Delta\left(\mathrm{CO}_{2} /\right.$ energy $)$ in the first period for the two provinces probably is due to the increasing of raw coal consumption. The reason for the pattern of $\mathrm{CO}_{2}$ /energy should be the same as CQ and JX. Conversely, these two aggregated provinces reduce the raw coal energy consumption percentage in the first period, but increase in the second period.

\section{Decarbonisation}

In light of the BIC index in Figure 7-10, model EEV with 10 components is the best model based cluster. Figure 7-12 illustrates GMM and AHC results based on the changes of $\mathrm{CO}_{2} / \mathrm{GDP}$ during the three periods. Figure $7-15$ presents the value of changes of $\mathrm{CO}_{2} / \mathrm{GDP}$. For these three periods, the $\mathrm{CO}_{2}$ intensity decreased, except $\mathrm{HuB}$ and IM in the 11 FYP, HN, FJ, SD and YN in the 10th FYP, CQ, JX and NX in the 9th FYP . 


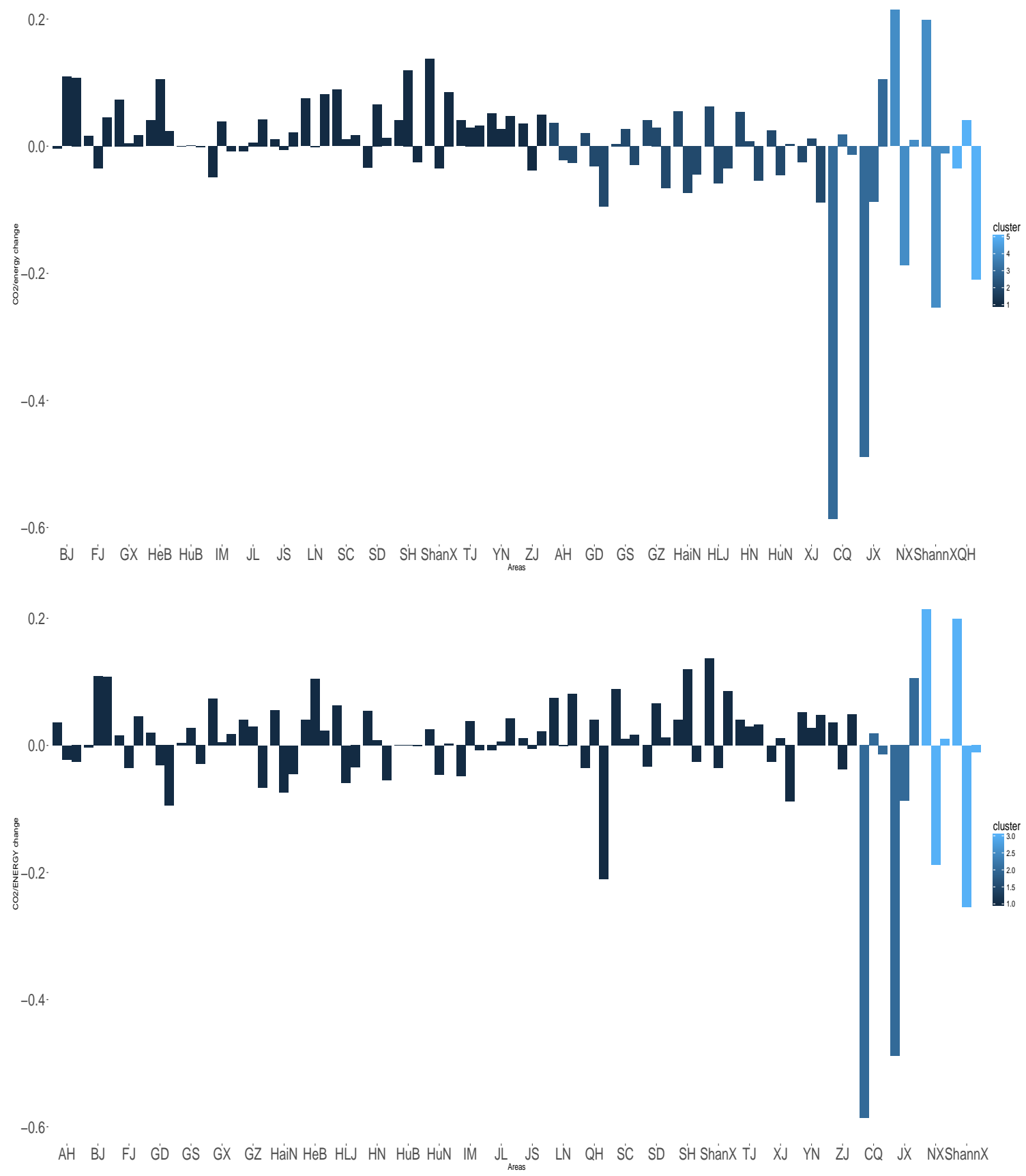

Figure 7-9: Indicator dematerialization at provincial-level during three periods based on AHC and GMM respectively 


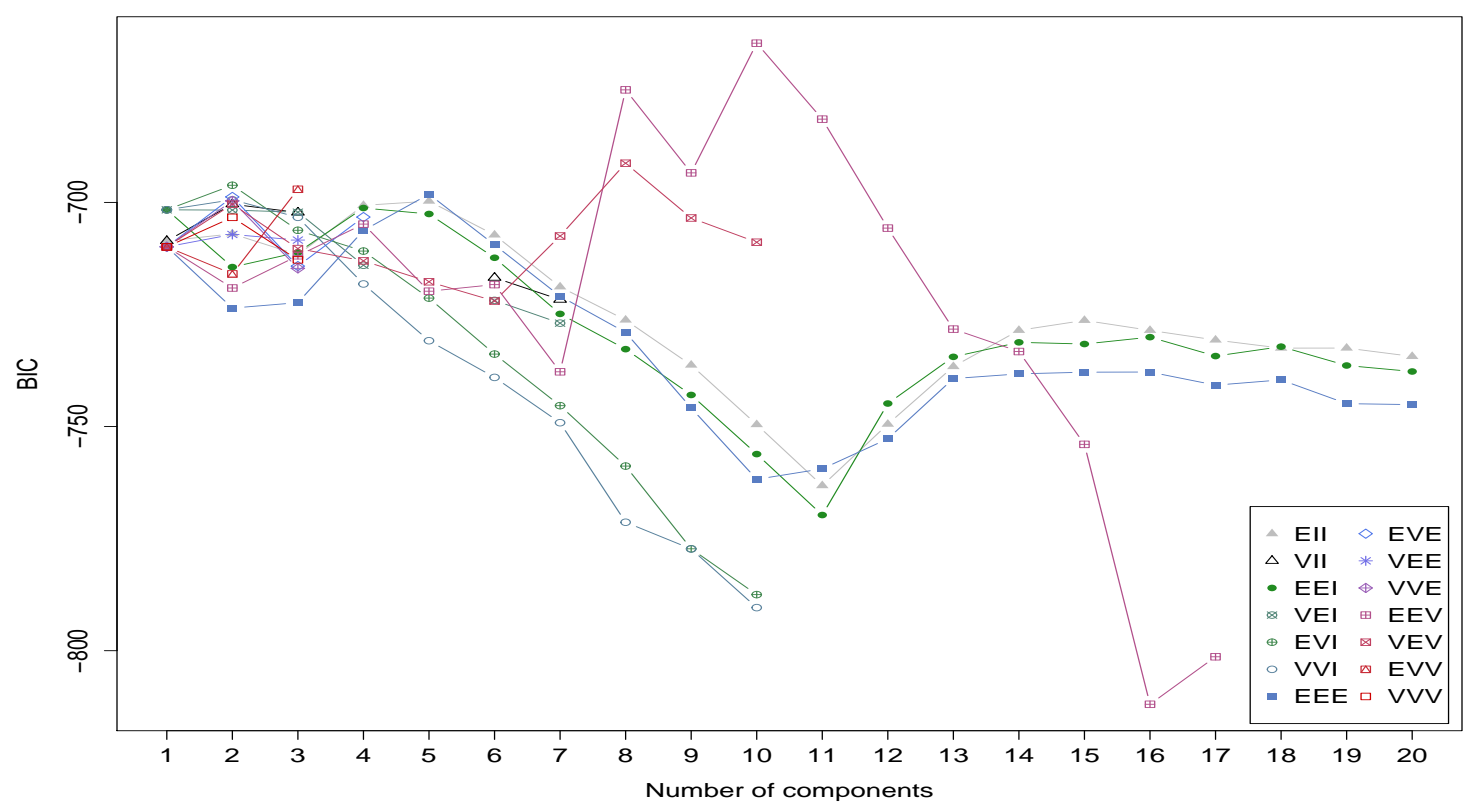

Figure 7-10: BIC for indicator dematerialization

\subsection{Driven factors analysis}

From the previous analysis, there are four provinces deserved to be discuss more, since for three indicators they shows distinct pattens from cluster analysis. They are IM, CQ, JX ad NX. IM is an outlier showing extremely different from others. For immaterialisation, IM increases its energy usage significantly without slight improve its economic in the 11 FYP. Conversely, CQ, JX and NX illustrate a considerably decreasing for energy intensity(energy/GDP). This is mainly due to the shares changes of services in the economy in these three province. During the time of $12 \mathrm{FYP}$, The changes of the added value of tertiary sector in CQ is 0.144 from 2006 to 2011, JX is 0.183 , and NX is 0.161 respectively. All are above the national average. But IM is only 0.023 as shown in Table 7.3 .

Regarding dematerialisation, the tremendous disparity is between cluster CQ JX, and cluster NX, ShannX. The indicator dematerialisation for the cluster including provinces CQ and JX is consistent with the patterns of changes in the raw coal percentage of total energy in this three provinces. This means the improvement of 

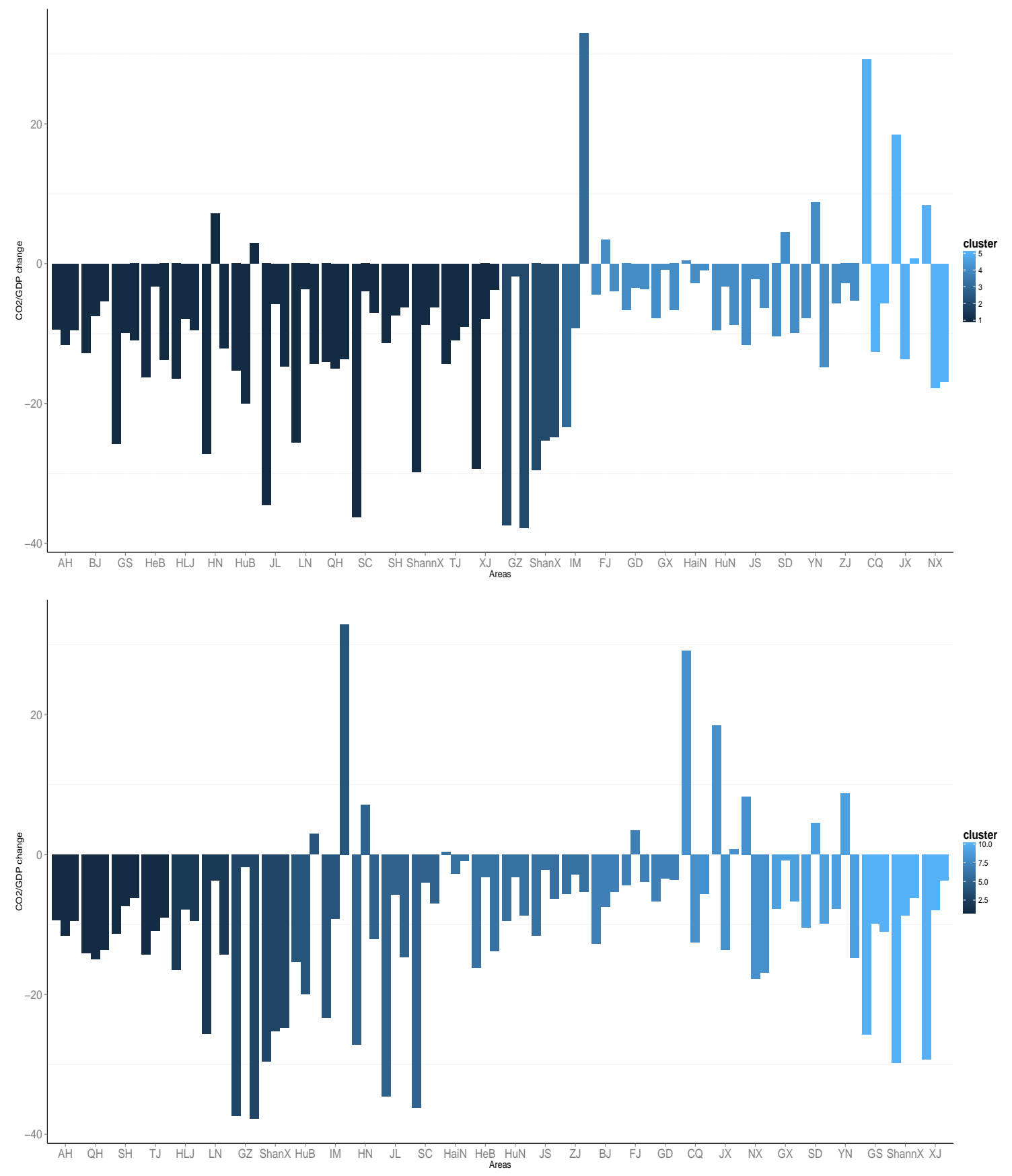

Figure 7-11: Indicator decarbonisation at provincial-level correspond to three periods based on AHC and GMM respectively 

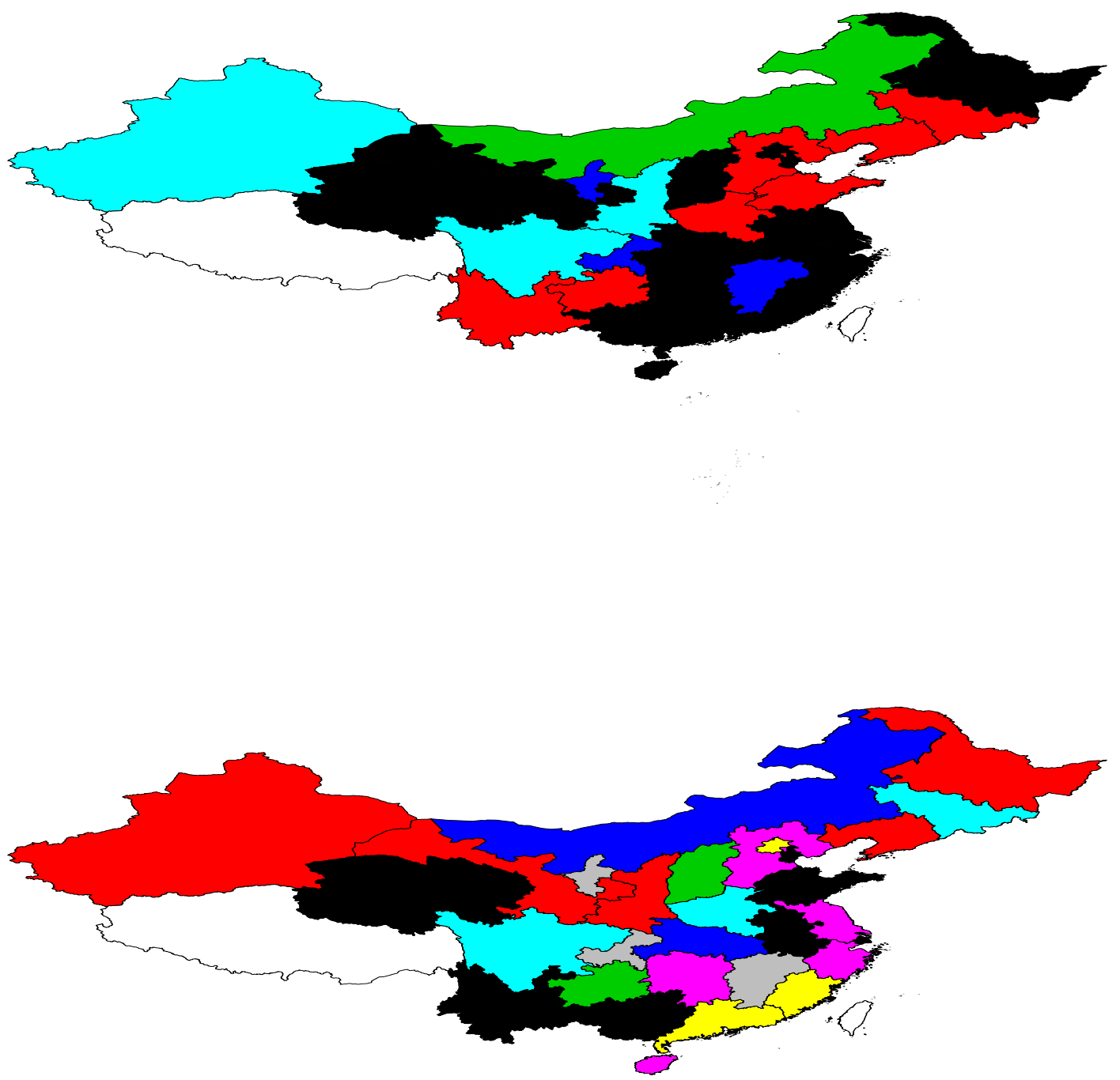

Figure 7-12: AHC results against GMM results for the dematerialization indicator 
Table 7.1: Changes in the percentage of added values of the primary, secondary, and tertiary sectors at provincial-level in China

\begin{tabular}{|c|c|c|c|c|c|c|c|c|c|}
\hline & 2001-1996 & $\begin{array}{c}2006-2001 \\
\text { Primary }\end{array}$ & 2011-2006 & 2001-1996 & $\begin{array}{l}\text { 2006-2001 } \\
\text { Secondary }\end{array}$ & $2011-2006$ & 2001-1996 & $\begin{array}{c}\text { 2006-2001 } \\
\text { Tertiary }\end{array}$ & 2011-2006 \\
\hline BJ & -0.4687 & -0.4979 & -0.3524 & -0.2287 & -0.1238 & -0.2774 & 0.1976 & 0.0731 & 0.1095 \\
\hline TJ & -0.3198 & -0.4355 & -0.4722 & -0.0795 & 0.1017 & -0.1760 & 0.1574 & -0.0718 & 0.2530 \\
\hline $\mathrm{HeB}$ & -0.1840 & -0.2304 & -0.1337 & 0.0140 & 0.0901 & -0.0637 & 0.0972 & -0.0170 & 0.1501 \\
\hline ShanX & -0.4506 & -0.3270 & -0.1018 & 0.0141 & 0.1991 & -0.0668 & 0.1639 & -0.1489 & 0.1150 \\
\hline IM & -0.3151 & -0.3868 & -0.3471 & 0.0731 & 0.2555 & 0.0740 & 0.2082 & -0.0411 & 0.0231 \\
\hline LN & -0.2795 & -0.0666 & -0.2441 & -0.0042 & 0.0122 & -0.0136 & 0.1213 & 0.0032 & 0.0768 \\
\hline JL & -0.3089 & -0.1844 & -0.3000 & 0.0083 & 0.1143 & 0.0801 & 0.2576 & -0.0256 & 0.0287 \\
\hline HLJ & -0.3143 & -0.0602 & 0.0212 & -0.0240 & 0.0357 & -0.2024 & 0.2594 & -0.0314 & 0.3173 \\
\hline SH & -0.3557 & -0.4073 & -0.3552 & -0.1456 & 0.0192 & -0.2278 & 0.1989 & -0.0052 & 0.2115 \\
\hline JS & -0.2975 & -0.3860 & -0.2224 & 0.0136 & 0.0887 & -0.1957 & 0.1302 & -0.0036 & 0.3471 \\
\hline ZJ & -0.3266 & -0.3846 & -0.2547 & -0.0281 & 0.0455 & -0.1528 & 0.1889 & 0.0342 & 0.2445 \\
\hline $\mathrm{AH}$ & -0.2662 & -0.2941 & -0.2789 & 0.0903 & 0.1476 & 0.1087 & 0.1625 & 0.0313 & -0.0054 \\
\hline FJ & -0.2610 & -0.2857 & -0.2702 & 0.0715 & 0.1003 & -0.0378 & 0.0726 & 0.0032 & 0.1237 \\
\hline JX & -0.2549 & -0.2988 & -0.3383 & 0.0583 & 0.3892 & -0.0124 & 0.1721 & -0.1752 & 0.1832 \\
\hline SD & -0.2752 & -0.3394 & -0.2055 & 0.0471 & 0.1588 & -0.1832 & 0.1048 & -0.0799 & 0.3817 \\
\hline $\mathrm{HN}$ & -0.1352 & -0.3050 & -0.2552 & -0.0170 & 0.1988 & -0.0675 & 0.1523 & -0.0686 & 0.2534 \\
\hline $\mathrm{HuB}$ & -0.3776 & -0.1607 & -0.2325 & 0.0980 & 0.0888 & -0.0063 & 0.2093 & -0.0178 & 0.0920 \\
\hline $\mathrm{HuN}$ & -0.3106 & -0.2321 & -0.2467 & 0.0179 & 0.1243 & 0.0170 & 0.2786 & 0.0101 & 0.0804 \\
\hline GD & -0.3997 & -0.2984 & -0.2242 & -0.0549 & 0.1077 & -0.1243 & 0.2143 & -0.0538 & 0.1742 \\
\hline GX & -0.1974 & -0.1397 & -0.2844 & -0.0220 & 0.1699 & 0.0903 & 0.2058 & -0.0542 & 0.0675 \\
\hline HaiN & -0.0620 & -0.1066 & -0.2804 & 0.1046 & 0.2532 & -0.1825 & 0.0013 & -0.0520 & 0.3391 \\
\hline $\mathrm{CQ}$ & -0.3178 & -0.3371 & -0.3088 & -0.0156 & 0.1247 & -0.0635 & 0.2186 & -0.0067 & 0.1444 \\
\hline $\mathrm{SC}$ & -0.1473 & -0.1970 & -0.3104 & -0.0905 & 0.1865 & 0.0774 & 0.2306 & -0.0573 & 0.0611 \\
\hline GZ & -0.3120 & -0.3254 & -0.3397 & 0.0845 & 0.0814 & -0.2121 & 0.2710 & 0.1270 & 0.3386 \\
\hline YN & -0.1250 & -0.1261 & -0.2402 & -0.0791 & 0.0536 & -0.1355 & 0.2007 & 0.0115 & 0.2601 \\
\hline ShannX & -0.3555 & -0.3511 & -0.4103 & 0.3187 & 0.1991 & 0.0507 & 0.2291 & 0.0978 & 0.1053 \\
\hline GS & -0.3638 & -0.2205 & -0.1460 & 0.0334 & 0.1828 & -0.0410 & 0.1641 & -0.1181 & 0.0949 \\
\hline $\mathrm{QH}$ & -0.2903 & -0.2063 & -0.2017 & -0.0573 & 0.1254 & -0.1046 & 0.3259 & -0.0316 & 0.1960 \\
\hline NX & -0.3206 & -0.3012 & -0.2149 & 0.0966 & 0.2280 & 0.0048 & 0.0840 & -0.1154 & 0.0519 \\
\hline XJ & -0.3099 & -0.2556 & -0.2841 & 0.0145 & 0.2027 & -0.0711 & 0.1548 & -0.0978 & 0.1614 \\
\hline Average & -0.2905 & -0.2661 & -0.2578 & 0.0134 & 0.1357 & -0.0726 & 0.1793 & -0.0341 & 0.1693 \\
\hline
\end{tabular}




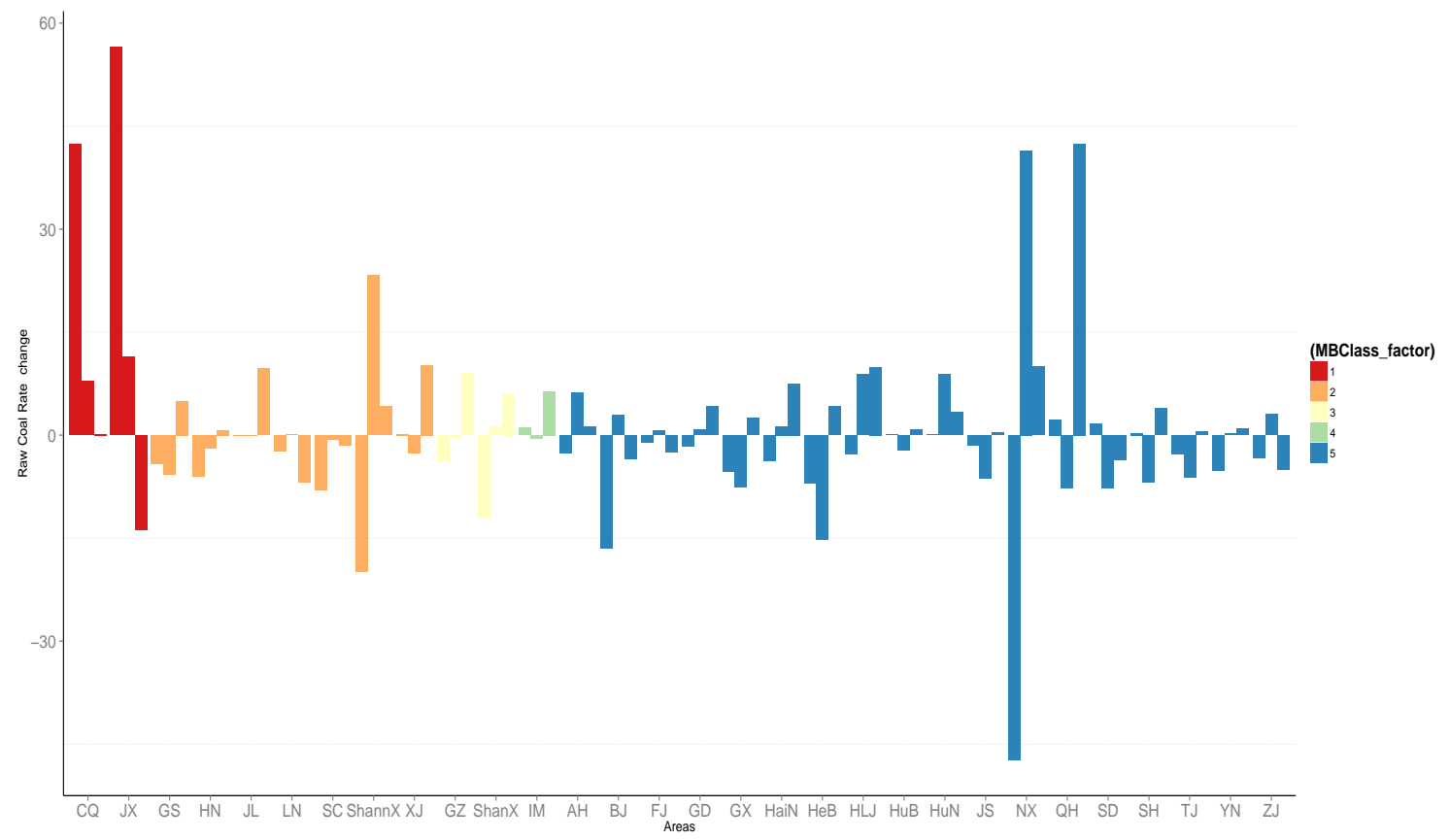

Figure 7-13: Changes in the raw coal percentage of total energy in the three periods corresponding to different provinces.

energy efficiency in CQ and JX due to the transferring of energy usage structure from using raw coal to cleaner energy sources.

Table 7.3 and Table 7.3 illustrate the increasing rate of investment in cold mining and processing industry, and energy industry. It is interesting to see that in the period of 2006 to 2001, IM speed up its investment in the secondary industry sector.

It is also interesting to see the correlation between the effects of the urbanization rate or population on energy usage and emission as mentioned in the studies [237, 238]. The same conclusion cannot be obtained in China. Figure 7-14 lists the urbanization rate increasing between 2011 and 2005. It can be seen that the urbanization rate increasing of provinces GZ, HN, and ShannX are much higher than the ones in other areas. Therefore, the urbanization probably is not primary factor that distinguishes the patterns among the clusters for neither of the three indicators.

According to the specific factors, study [90] illustrated the switching in power generation from coal to natural gas in UK and increasing in industrial and power plant efficiency of eastern Germany is the main reason to discriminate from other European 
Table 7.2: Increasing rate of investment in coal mining and processing industry

\begin{tabular}{|c|c|c|c|c|c|}
\hline 2001-1996 & & 2006-2001 & & 2011-2006 & \\
\hline IM & -87.925 & $\mathrm{SD}$ & 69.430 & $\mathrm{AH}$ & -7.478 \\
\hline BJ & -64.516 & BJ & 95.455 & $\mathrm{SD}$ & 6.037 \\
\hline $\mathrm{SC}$ & -62.952 & JS & 104.228 & JS & 25.203 \\
\hline NX & -56.425 & $\mathrm{LN}$ & 177.204 & $\mathrm{HN}$ & 116.265 \\
\hline GX & -53.846 & GS & 188.037 & $\mathrm{HeB}$ & 136.008 \\
\hline $\mathrm{HuB}$ & -53.448 & $\mathrm{HeB}$ & 252.495 & JL & 154.580 \\
\hline $\mathrm{JL}$ & -52.333 & JX & 300.752 & IM & 158.649 \\
\hline ShannX & -51.546 & HLJ & 326.774 & $\mathrm{CQ}$ & 166.716 \\
\hline XJ & -44.94 & FJ & 384.404 & $\mathrm{LN}$ & 183.326 \\
\hline $\mathrm{YN}$ & -44.293 & ShanX & 556.238 & $\mathrm{QH}$ & 191.886 \\
\hline $\mathrm{AH}$ & -34.986 & $\mathrm{AH}$ & 580.496 & NX & 209.615 \\
\hline $\mathrm{HuN}$ & -34.91 & $\mathrm{HN}$ & 778.819 & HLJ & 261.389 \\
\hline GZ & -33.534 & GX & 818.75 & ShanX & 305.824 \\
\hline $\mathrm{HN}$ & -32.787 & GZ & 906.787 & $\mathrm{YN}$ & 325.31 \\
\hline FJ & -26.351 & $\mathrm{HuN}$ & 939.091 & $\mathrm{XJ}$ & 328.259 \\
\hline HLJ & -22.113 & ShannX & 1038.771 & GX & 387.302 \\
\hline $\mathrm{QH}$ & -16.667 & YN & 1120 & $\mathrm{SC}$ & 437.95 \\
\hline JS & -11.688 & $\mathrm{HuB}$ & 1270.370 & BJ & 527.907 \\
\hline $\mathrm{HeB}$ & -5.088 & NX & 1553.419 & GS & 626.087 \\
\hline ShanX & -1.606 & XJ & 1608.108 & JX & 636.9611 \\
\hline $\mathrm{LN}$ & 30.378 & $\mathrm{SC}$ & 1807.724 & $\mathrm{HuN}$ & 696.807 \\
\hline GS & 31.450 & $\mathrm{JL}$ & 2320.280 & GZ & 708.921 \\
\hline JX & 43.011 & $\mathrm{QH}$ & 2365 & FJ & 726.894 \\
\hline SD & 106.295 & CQ & 2616.129 & ShannX & 952.751 \\
\hline CQ & 12400 & IM & 5242.488 & $\mathrm{HuB}$ & 1132.432 \\
\hline
\end{tabular}


Table 7.3: Increasing rate of investment in Energy Industry corresponding to different periods and provinces

\begin{tabular}{|c|c|c|c|c|c|}
\hline 2001-1996 & & 2006-2001 & & 2011-2006 & \\
\hline HaiN & -54.481 & $\mathrm{SH}$ & 19.068 & $\mathrm{SH}$ & 5.914 \\
\hline BJ & -27.461 & $\mathrm{HuB}$ & 49.099 & ZJ & 19.355 \\
\hline $\mathrm{AH}$ & -24.6811 & HLJ & 105.745 & $\mathrm{AH}$ & 30.044 \\
\hline IM & -10.174 & $\mathrm{SD}$ & 108.887 & GD & 30.127 \\
\hline $\mathrm{SC}$ & -5.2127 & $\mathrm{LN}$ & 119.521 & JS & 35.082 \\
\hline GD & 21.453 & ZJ & 126.248 & BJ & 35.652 \\
\hline $\mathrm{LN}$ & 23.384 & JX & 133.9269 & $\mathrm{CQ}$ & 40.918 \\
\hline HLJ & 28.221 & XJ & 144.531 & $\mathrm{HuB}$ & 42.05 \\
\hline SH & 41.005 & BJ & 146.293 & $\mathrm{HN}$ & 44.042 \\
\hline $\mathrm{HN}$ & 41.426 & FJ & 163.644 & GX & 47.934 \\
\hline TJ & 59.72 & $\mathrm{QH}$ & 166.153 & $\mathrm{SD}$ & 48.09 \\
\hline $\mathrm{HuN}$ & 65.6740 & JS & 173.111 & TJ & 59.737 \\
\hline YN & 66.496 & $\mathrm{HuN}$ & 176.839 & HaiN & 74.08 \\
\hline $\mathrm{HuB}$ & 67.832 & GS & 179.261 & YN & 81.67 \\
\hline $\mathrm{HeB}$ & 73.223 & TJ & 184.892 & $\mathrm{HeB}$ & 87.843 \\
\hline FJ & 78.513 & $\mathrm{HN}$ & 205.238 & $\mathrm{QH}$ & 97.431 \\
\hline JS & 79.683 & GZ & 205.672 & IM & 102.229 \\
\hline GS & 81.901 & $\mathrm{JL}$ & 221.288 & FJ & 110.417 \\
\hline JL & 82.369 & GD & 223.309 & JX & 113.125 \\
\hline XJ & 87.062 & $\mathrm{HeB}$ & 269.718 & $\mathrm{HuN}$ & 121.929 \\
\hline SD & 87.322 & ShannX & 306.044 & HLJ & 127.867 \\
\hline $\mathrm{QH}$ & 98.339 & ShanX & 327.785 & $\mathrm{SC}$ & 129.414 \\
\hline NX & 106.814 & GX & 376.265 & $\mathrm{LN}$ & 130.109 \\
\hline ShanX & 119.162 & NX & 389.951 & $\mathrm{JL}$ & 136.647 \\
\hline GX & 123.094 & $\mathrm{SC}$ & 392.32 & GZ & 140.646 \\
\hline ShannX & 126.029 & HaiN & 435.235 & XJ & 151.704 \\
\hline JX & 162.268 & $\mathrm{CQ}$ & 477.667 & ShanX & 181.361 \\
\hline ZJ & 180.682 & $\mathrm{AH}$ & 532.666 & ShannX & 221.865 \\
\hline GZ & 252.374 & YN & 737.447 & NX & 248.083 \\
\hline CQ & 320 & IM & 1321.096 & GS & 268.534 \\
\hline
\end{tabular}




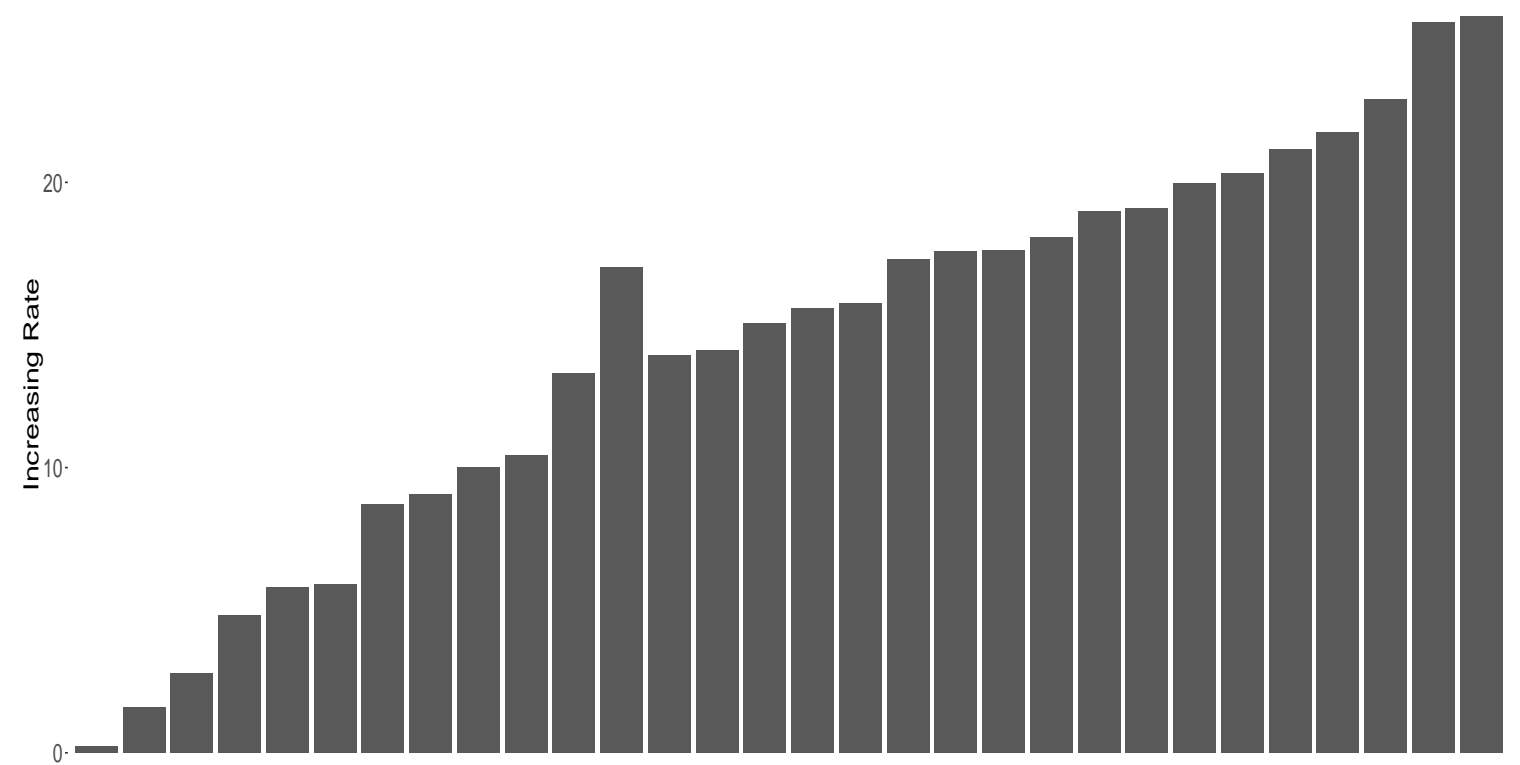

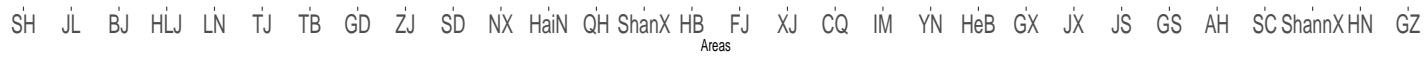

Figure 7-14: The urbanization rate corresponding to different areas from year 2005 to 2013.

countries considering immaterialisation indicator. Also, the authors mentioned the share of different industries is main factor to explain the differences among clusters. However, this study fails to explain the possible reasons of the cluster differences for decarbonisation indicator.

\subsection{Discussion}

In this study, the provinces in China have been clustered by the indicators immaterialisation, dematerialization, and decarbonisation in order to understand the similar pattern in the same cluster, and the different patterns among the clusters. For each of the indicator, two extremely disparities clusters and their possible driven factors were analyzed and discussed. Some preliminary results are summarised as follows:

- The provinces in China have been grouped by two cluster algorithms based on three indicators: immaterialisation, dematerialization, and decarbonisation that are characterized dynamically representation of the interaction among the 


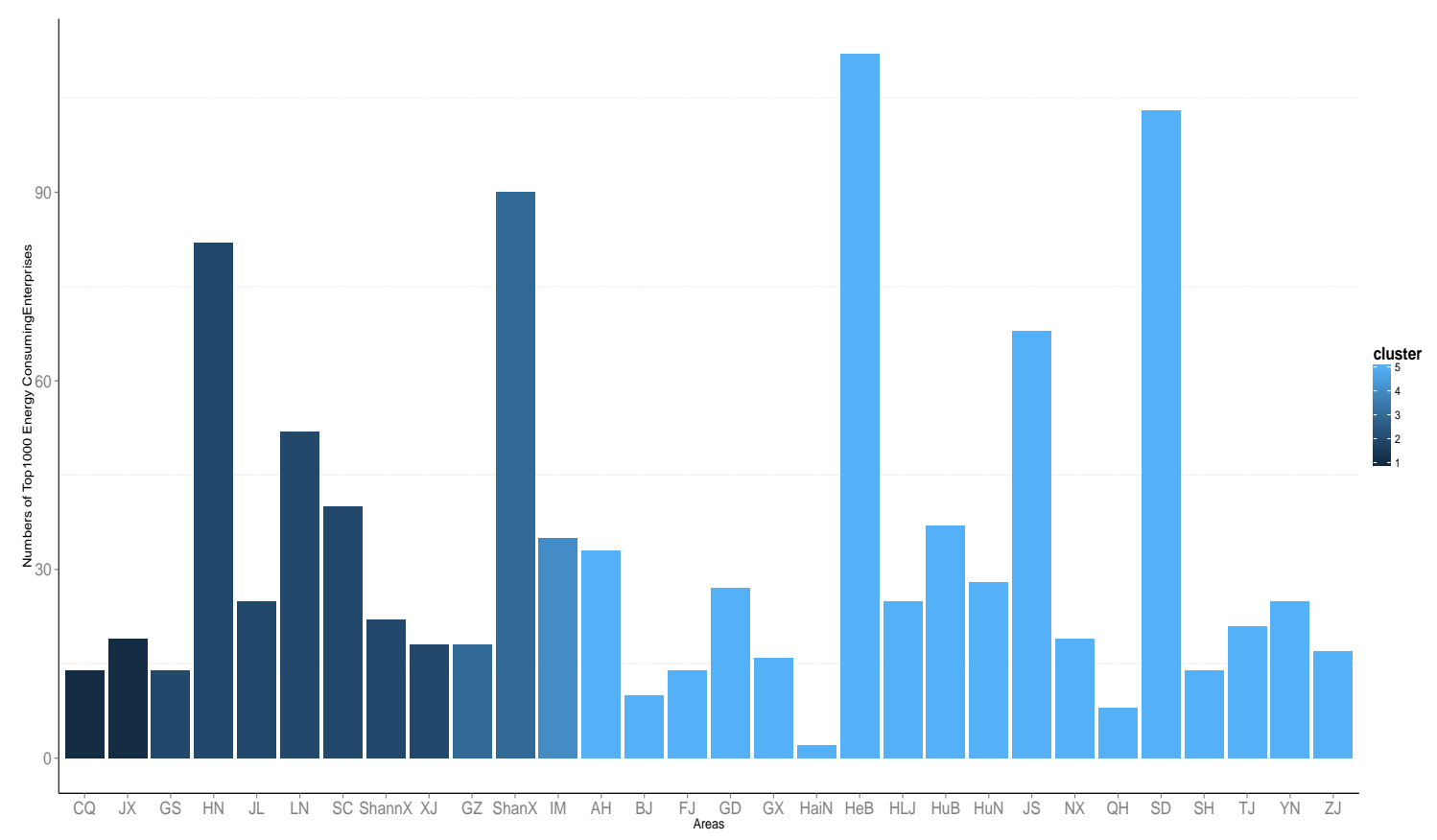

Figure 7-15: Top 1000 energy consumption enterprises corresponding to the provinces in 2004 .

energy usage, $\mathrm{CO}_{2}$ emission, and GDP.

- Results show that both two algorithms can successfully separate the provinces with biggest differences. For instance, cluster with IM and cluster CQ,JX and NX for indicator immaterialisation.

- From the description analysis, the driven factor for immaterialisation could be the changes of the added value of primary, secondary, and tertiary sectors. For dematerialization, the influencing factor could be the changes in the raw coal usage in China.

- Other factors such as the changes in urbanization rate is not responsible for the differences of the clusters.

However, all the analysis of identifying driven factors are based on the description analysis. More factors are necessary to be considered further. For instance, the 13 macro influential factors according to Kaya identity as shown in Table 7.4 were taken into account from the studies[91, 237]. Kaya identity is a special tool provided by 
Table 7.4: Potential influential factors to $\mathrm{CO}_{2}$ emission.

Index

Population (\%)

Per capita added value of the primary industry $(\%)$

Primary industry energy consumption of the per-unit added value(\%)

Primary industry carbon emission of the per-unit energy consumption(\%)

Per capita added value of the secondary industry(\%)

Secondary industry energy consumption of the per-unit added value(\%)

Secondary industry carbon emission of the per-unit energy consumption(\%)

Per capita added value of the tertiary industry(\%)

Tertiary industry energy consumption of the per-unit added value(\%)

Tertiary industry carbon emission of the per-unit energy consumption(\%)

Per capita resident income

Energy consumption of the per-unit resident income(\%)

Carbon emission of the per-unit resident energy consumption(\%)

IPCC to reveal the importance of distinct components or factors in the historical data of energy-related $\mathrm{CO}_{2}$ emission. Integrating the Kaya identity to identify the driven factors for each of the three indicators by modeling is one of the future research activities. 
Table 7.5: Values of immaterialisation, decarbonisation and dematerialization for each province.

\begin{tabular}{|c|c|c|c|c|c|c|c|c|c|}
\hline & \multicolumn{3}{|c|}{ immaterialisation } & \multicolumn{3}{|c|}{ decarbonisation } & \multicolumn{3}{|c|}{ dematerialization } \\
\hline & 2001- & 2006- & 2011- & 2001- & 2006- & 2011- & 2001- & 2006- & 2011- \\
\hline & 1996 & 2001 & 2006 & 1996 & 2001 & 2006 & 1996 & 2001 & 2006 \\
\hline BJ & -6.32 & -3.98 & -2.66 & -12.74 & -7.45 & -5.38 & -0.00 & 0.11 & 0.11 \\
\hline TJ & -7.49 & -5.51 & -4.44 & -14.31 & -10.91 & -8.99 & 0.04 & 0.03 & 0.03 \\
\hline $\mathrm{HeB}$ & -9.58 & -3.00 & -7.25 & -16.25 & -3.26 & -13.77 & 0.04 & 0.11 & 0.02 \\
\hline ShanX & -27.49 & -15.78 & -18.30 & -29.56 & -25.25 & -24.80 & 0.14 & -0.04 & 0.09 \\
\hline IM & -11.76 & -5.84 & 18.28 & -23.34 & -9.23 & 32.92 & -0.05 & 0.04 & -0.01 \\
\hline $\mathrm{LN}$ & -13.80 & -1.79 & -7.46 & -25.60 & -3.67 & -14.32 & 0.08 & -0.00 & 0.08 \\
\hline JL & -18.16 & -3.12 & -8.01 & -34.56 & -5.77 & -14.67 & -0.01 & 0.01 & 0.04 \\
\hline HLJ & -9.19 & -3.45 & -4.72 & -16.47 & -7.82 & -9.51 & 0.06 & -0.06 & -0.03 \\
\hline $\mathrm{SH}$ & -5.61 & -3.89 & -2.72 & -11.28 & -7.41 & -6.23 & 0.04 & 0.12 & -0.03 \\
\hline JS & -6.07 & -1.09 & -3.32 & -11.61 & -2.17 & -6.29 & 0.01 & -0.01 & 0.02 \\
\hline $\mathrm{ZJ}$ & -3.02 & -1.23 & -2.82 & -5.63 & -2.79 & -5.30 & 0.04 & -0.04 & 0.05 \\
\hline $\mathrm{AH}$ & -5.50 & -5.97 & -4.95 & -9.38 & -11.57 & -9.46 & 0.04 & -0.02 & -0.03 \\
\hline FJ & -2.21 & 1.84 & -2.08 & -4.36 & 3.45 & -3.88 & 0.02 & -0.04 & 0.05 \\
\hline JX & 10.26 & -6.72 & 0.03 & 18.47 & -13.64 & 0.75 & -0.49 & -0.09 & 0.11 \\
\hline $\mathrm{SD}$ & -5.32 & 1.91 & -5.26 & -10.41 & 4.49 & -9.90 & -0.03 & 0.07 & 0.01 \\
\hline $\mathrm{HuN}$ & -5.47 & -1.37 & -4.89 & -9.52 & -3.25 & -8.75 & 0.03 & -0.05 & 0.00 \\
\hline $\mathrm{HuB}$ & -7.81 & -10.21 & 1.52 & -15.32 & -20.00 & 2.97 & -0.00 & 0.00 & -0.00 \\
\hline $\mathrm{HN}$ & -14.78 & 3.64 & -6.03 & -27.20 & 7.14 & -12.07 & 0.05 & 0.01 & -0.05 \\
\hline GD & -3.11 & -1.47 & -1.46 & -6.65 & -3.43 & -3.61 & 0.02 & -0.03 & -0.09 \\
\hline GX & -4.47 & -0.46 & -3.40 & -7.75 & -0.86 & -6.65 & 0.07 & 0.00 & 0.02 \\
\hline HaiN & 0.03 & -1.00 & -0.31 & 0.41 & -2.73 & -0.92 & 0.05 & -0.07 & -0.04 \\
\hline CQ & 15.99 & -6.79 & -2.92 & 29.20 & -12.57 & -5.64 & -0.59 & 0.02 & -0.01 \\
\hline $\mathrm{SC}$ & -20.48 & -2.12 & -3.68 & -36.26 & -3.96 & -6.96 & 0.09 & 0.01 & 0.02 \\
\hline GZ & -22.34 & -1.64 & -19.97 & -37.44 & -1.76 & -37.76 & 0.04 & 0.03 & -0.07 \\
\hline YN & -4.78 & 4.48 & -8.20 & -7.78 & 8.76 & -14.77 & 0.05 & 0.03 & 0.05 \\
\hline ShannX & -17.80 & -2.55 & -3.41 & -29.79 & -8.69 & -6.25 & 0.20 & -0.25 & -0.01 \\
\hline GS & -13.33 & -5.37 & -5.36 & -25.78 & -9.90 & -10.97 & 0.00 & 0.03 & -0.03 \\
\hline $\mathrm{QH}$ & -6.82 & -8.21 & -5.92 & -14.07 & -15.01 & -13.60 & -0.03 & 0.04 & -0.21 \\
\hline NX & -1.60 & -4.73 & -10.04 & 8.33 & -17.80 & -16.92 & 0.21 & -0.19 & 0.01 \\
\hline XJ & -14.41 & -4.11 & -1.15 & -29.35 & -7.90 & -3.75 & -0.03 & 0.01 & -0.09 \\
\hline
\end{tabular}




\section{Chapter 8}

\section{Conclusion and Future work}

\subsection{Conclusion}

This study aims to use the sophisticated artificial intelligence and statistic techniques to forecast pollution and assess its social impact. Under this target, several subtopics are implemented by different cases studies on the application areas of environment and energy. Then, the conclusions can be summarized as following:

1 A framework is proposed for relocating and reconfiguring the existing pollution monitoring station networks by using feature selection and artificial intelligence techniques. This methodology enables a partial redesign based on the maximization of the available information gathered by the pollution networks, but it also considers requirements from the decision makers, like potential target place, etc. The methodology is based on the quality of forecasting by AI-based models.

A case study is presented in Chapter 4 showing that the state-of-the-art machine learning algorithm, RF, can improve model performance significantly compared to the standard application of MRL and ANN. Compare to PCA deteriorating the forecasting accuracy, SA and RFE feature selection implemented with RF models can reduce the number of features, select the optimal combination of input features, and build simpler models without losing forecasting accuracy. 
The forecasting results are sensitive to the size of the samples, that is, increasing the size of the samples can improve the performance of the models, which contributes to the robustness of the system. Through the feature selection and comparison of the AI models, the maximum information can be gathered by using the best prediction of FS and AI model. The selection feature selection and AI model can also be useful for auditing and forecasting over time. In light of the prediction results, a new way of relocating the existing monitoring station is proposed.

2 A comprehensive assessment of the influences factors of socio-economic activities on pollution at district level in the macro environment is presented. The socio-economic activity factors include five categories, namely manufacturer, construction, traffic and transportation, population and household structure, and meteorology. The pollutants involves $\mathrm{NO}_{x}, \mathrm{NO}_{2}, \mathrm{O}_{3}, \mathrm{RSP}$ and $\mathrm{SO}_{2}$. Instead of the questionnaire survey and measuring emission at sampling site directly, this study uses the statistic data from Hong Kong annual report in different sectors and pollution records from monitoring stations. Also, it adopted econometrics method, fixed-effect model, and random effect model, to explore each pollutant formation mechanism from the socio-economic activity perspective.

The results show that the manufacturer, construction activities, and the population and household structure play a more important role than the traffic flow in the formation of $\mathrm{NO}_{x}$ and $\mathrm{NO}_{2}$. However, $\mathrm{SO}_{2}$ cannot be explained by these factors. The other sector activities, such as energy generation from the power plant, could dominate the $\mathrm{SO}_{2}$ emission. RSP, on the other hand, is formatted by the construction activity and regional transportation than local vehicle emission. Meteorological factors, especially $\mathrm{NO}_{x}$ and $\mathrm{NO}_{2}$, are significant contributors to the studied pollutants. As the significant association between WD and WS on $\mathrm{NO}_{x}, \mathrm{NO}_{2}, \mathrm{RSP}$ and $\mathrm{O}_{3}$ is strong, and this study believes that pollution transportation through wind factors plays an import role for these pollutants. The results can effectively help to select the potential input vari- 
ables for the local pollution prediction in Hong Kong area.

3 An innovative method of pollution forecasting, including regional factors, has been presented in Chapter 5. This method has applied on Hong Kong area to predict daily $\mathrm{O}_{3}$ threshold exceedances. The result shows that it can effectively address the imbalanced data problem when it predicts the rare cases - threshold exceedances, and improve the classifier's accuracy. By comparing the single-based data set without regional factors to the distance-based data set with regional factors, the experiments clearly illustrate that distance-based data set can capture ozone transportation characteristics and facilitate better forecasting. The Hong Kong authorities can promote the results of this research for improving the existing forecasting tools. Moreover, the findings can facilitate researchers' selection of the appropriate techniques in their future research.

4 This research provides a way to develop a multi-level awareness system of the energy used during production processes. The core of the multi-level awareness of energy is identifying the amount of energy used, $\mathrm{CO}_{2}$ emitted, and the cost of the energy used at operation, product, and order level. This system is achieved by integrating energy usage data with production data at the operational level. Furthermore, energy sources need to be considered to define the amount of $\mathrm{CO}_{2}$ that is emitted from the production process for each product. The relevant technique requirements are proposed and introduced making this system implementing feasible.

A pilot study in Chapter 6 was carried out to explain how to integrate electrical energy data, production data and scheduling data in real time to achieve the multi-level awareness of energy used in production. The results indicate that such system can provide energy consumption information to the stakeholders at different levels.

5 The provinces in China have been grouped by two cluster algorithms based on three indicators: immaterialisation, dematerialization, and decarbonisation that are characterized dynamically representation of the interaction among the 
energy usage, $\mathrm{CO}_{2}$ emission, and GDP in Chapter 7. The aim is to find different patterns among the provinces and identify the driven factors for the differences. The results show that both two algorithms can successfully separate the provinces with biggest differences. The possible driven factors have been analyzed. The changes in the added value of the primary, secondary and tertiary industries should be responsible for the changes of energy intensity(immaterialisation). The changing in the structures of raw coal is the main factor that drives the different patterns of the changing in energy efficiency(dematerialization). Another factor such as the changes in urbanization rate is not key one to explain the differences among the clusters.

\subsection{Future work}

In this research, several findings have been identified through the work carried out in different types of problems. Despite this, several other new issues come out, but they do not fit into available time framework for this research. Therefore, the future work regarding the new proposal, new methods, and more data analysis are needed to be done, as explained in the follows:

- Deep Learning for forecasting: An additional research path to a followup study is to compare all the AI techniques used in the intensive work of feature preparation and model establishment in this research with the new deeplearning (DL) techniques. The main method of feature selection is to manually design feature descriptors, which was also adopted in Chapter 4 and Chapter 5. One merit of DL is that, instead of the manual selection of features, it can extract features automatically from the original data sets. However, there are few studies on the application of DL for pollution forecasting. Since a sufficient number of available samples is required, both cases in this research could not employ DL for further research. Nevertheless, with the advent of the Internet of Things (IoT) and the proliferation of sensors that act as mobile pollution and meteorological monitoring stations, a large volume of data will be generated 
shortly. The accuracy of pollution forecasting models will benefit from and be realized by both IoT techniques.

- Heteorogeneous Data sources: Besides IoT, with the popularization of social media, a large amount of unstructured, free-text information about the quality of air pollution, quality of health are available. The messages cannot only act as a replacement of air pollution sensors as introduced before but also can generate more valuable information by analysis techniques. Sentiment analysis, one of these techniques, is compelling to understand the messages that we are interested- air pollution and the related heath impact.

Some studies evident that social media as social sensor can explore the relationship between the terms and air pollution somehow showing a strong relation between pollution and the volume of its related messages. However, few studies investigate the relationship between pollution and health outcome forecasting based on social media, which can be an effective way to help the hospital relocate the healthcare sources, then, avoid the patient churn, achieve near real-time surveillance of the pollution-related health emergency as well as assist the government to inform health intervention.

Therefore, one of the future research activities is to use heterogeneous data sources including pollution and meteorological data from the fix monitoring stations, and the messages with related keywords from social media(e.g. Twitter) to forecast daily hospital admission records by artificial intelligence techniques. This research is based on Madrid area. The data have been selected and described in Chapter 2, Section2.1.3. The modeling is undergoing, and the forecasting results are expected to be used in the daily hospital resources management.

- Health evaluation personalization: The pollution-related health studies always used ER functions in which the efficiency of each type of hospital admission corresponding to each type of pollution is based on survey. However, this efficiency should be changed according to different conditions such as various 
regions or age groups. For example, the mortality rate for the young people should be much lower than the rate for the other people as the older people are more sensitive. Integrate various data sources for individual-specific air quality modeling and pollution-related health modeling. The individuals under different health conditions or in different age cohorts have different pollution response. For example, the children, elder people or the ones who have cardiovascular disease are more sensitive to the pollution than the young people. Then, it need merge the individual information, such as the medical records with other data sources (pollution, meteorological, traffic, trajectory) for modeling in order to provide personal specific air quality information, pollution exposure awareness and give a pollution warning in real time.

In fact, the general idea of individual pollution exposure evaluation has been generated aiming to develop a system to provide pollution exposure information for specific users, based on which a pilot study is undergoing. The system is being developed and the framework is shown in Figure 8-1. The main data sources include air pollution and meteorology from outdoor fix monitoring stations, individual tracking data, and indoor pollution level from smart sensors. All the details has been depicted in Chapter 2, Section 2.1.3. The Kriging algorithm (Chapter 2, Section 2.2.3) is going to be used for the outdoor pollution interpolation, which plays a major role in the modeling. 

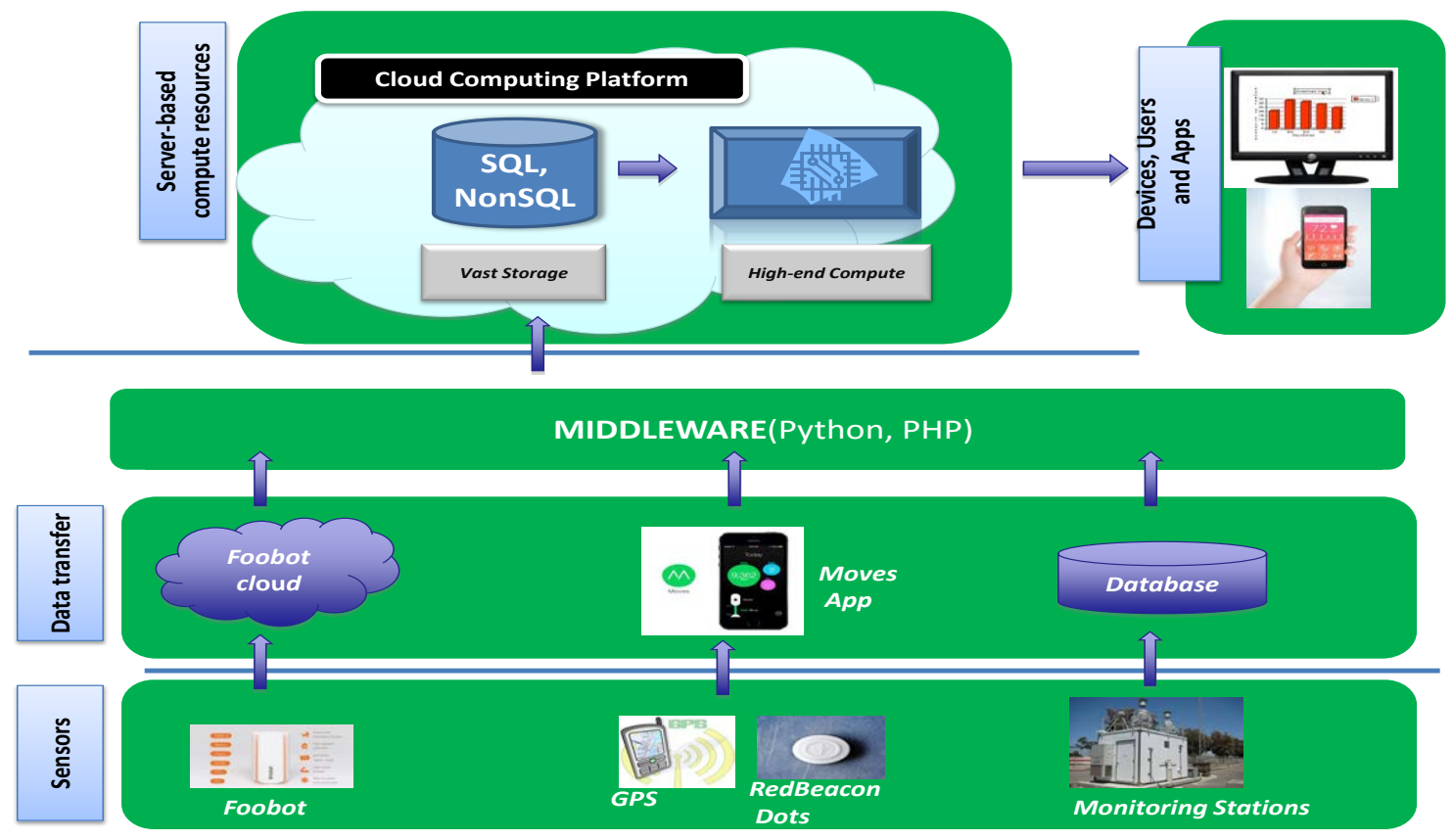

Figure 8-1: System design for individual pollution exposure evaluation. 


\section{Appendix A}

\section{Appendices}

\begin{tabular}{|c|c|c|c|}
\hline $\begin{array}{l}\text { Variable category } \\
\text { and data sources }\end{array}$ & Variables & $\begin{array}{l}\text { Abbrevi- } \\
\text { ation }\end{array}$ & Description \\
\hline \multirow[t]{2}{*}{ Description } & Industry area & IA & $\begin{array}{l}\text { 1:Located at industrial area, } 0 \text { : not } \\
\text { located at industrial area. }\end{array}$ \\
\hline & Year & Y & 2000 to 2007 \\
\hline $\begin{array}{l}\text { Manufacturer sec- } \\
\text { tor }\end{array}$ & $\begin{array}{l}\text { Numbers of estab- } \\
\text { lishment(NEM) }\end{array}$ & $\mathrm{NE}$ & $\begin{array}{l}\text { An establishment is defined as an } \\
\text { economic unit which engages, under a } \\
\text { single ownership or control, in one or } \\
\text { predominantly one kind of economic } \\
\text { activity at a single physical location. }\end{array}$ \\
\hline $\begin{array}{l}\text { Census and } \\
\text { Statistics De- } \\
\text { partment of Hong }\end{array}$ & $\begin{array}{l}\text { Numbers of employ- } \\
\text { ees }\end{array}$ & NEY & $\begin{array}{l}\text { Number of working proprietors, ac- } \\
\text { tive partners and unpaid family } \\
\text { workers + Number of direct employ- }\end{array}$ \\
\hline $\begin{array}{l}\text { Kong(Census and } \\
\text { Statistics Depart- }\end{array}$ & & & $\begin{array}{l}\text { ees, comprising operatives and other } \\
\text { employees }\end{array}$ \\
\hline
\end{tabular}

ment 2015) 


\begin{tabular}{|c|c|c|}
\hline $\begin{array}{l}\text { Compensation of } \\
\text { employees }\end{array}$ & CEY & $\begin{array}{l}\text { Wages and salaries (including share- } \\
\text { based payments) + Payments in kind } \\
\text { and employer's social security ex- } \\
\text { penditure (including contributions to } \\
\text { Mandatory Provident Fund schemes, } \\
\text { messing, medical expenses and pre- } \\
\text { miums for employees' compensation } \\
\text { insurance) }\end{array}$ \\
\hline $\begin{array}{l}\text { Purchases of ma- } \\
\text { terials supplies } \\
\text { and industrial } \\
\text { work/services }\end{array}$ & PMI & $\begin{array}{l}\text { Purchases of fuels }+ \text { Purchases of } \\
\text { electricity }+ \text { Purchases of water }+ \\
\text { Value of all other purchases of ma- } \\
\text { terials/supplies for production and } \\
\text { business operation + Payments for } \\
\text { sub-contract work + Payments for } \\
\text { repair and maintenance services }\end{array}$ \\
\hline Operation expense & $\mathrm{OE}$ & $\begin{array}{l}\text { Operating expenses = purchases of } \\
\text { materials, supplies and industrial } \\
\text { work/services + purchases of goods } \\
\text { for resale + rent, rates and govern- } \\
\text { ment rent for land and buildings + } \\
\text { rentals for machinery, equipment } \\
\text { and vehicles + interest payments + } \\
\text { miscellaneous operating expenses } \\
\text { (excluding bad debts, donations to } \\
\text { charities, fines, direct taxes, write-off, } \\
\text { depreciation, amortisation, provision, } \\
\text { obsolete stocks and loss on disposal } \\
\text { of fixed assets) - changes in stocks of } \\
\text { materials/supplies, work-in-progress, } \\
\text { finished products and goods for resale }\end{array}$ \\
\hline
\end{tabular}




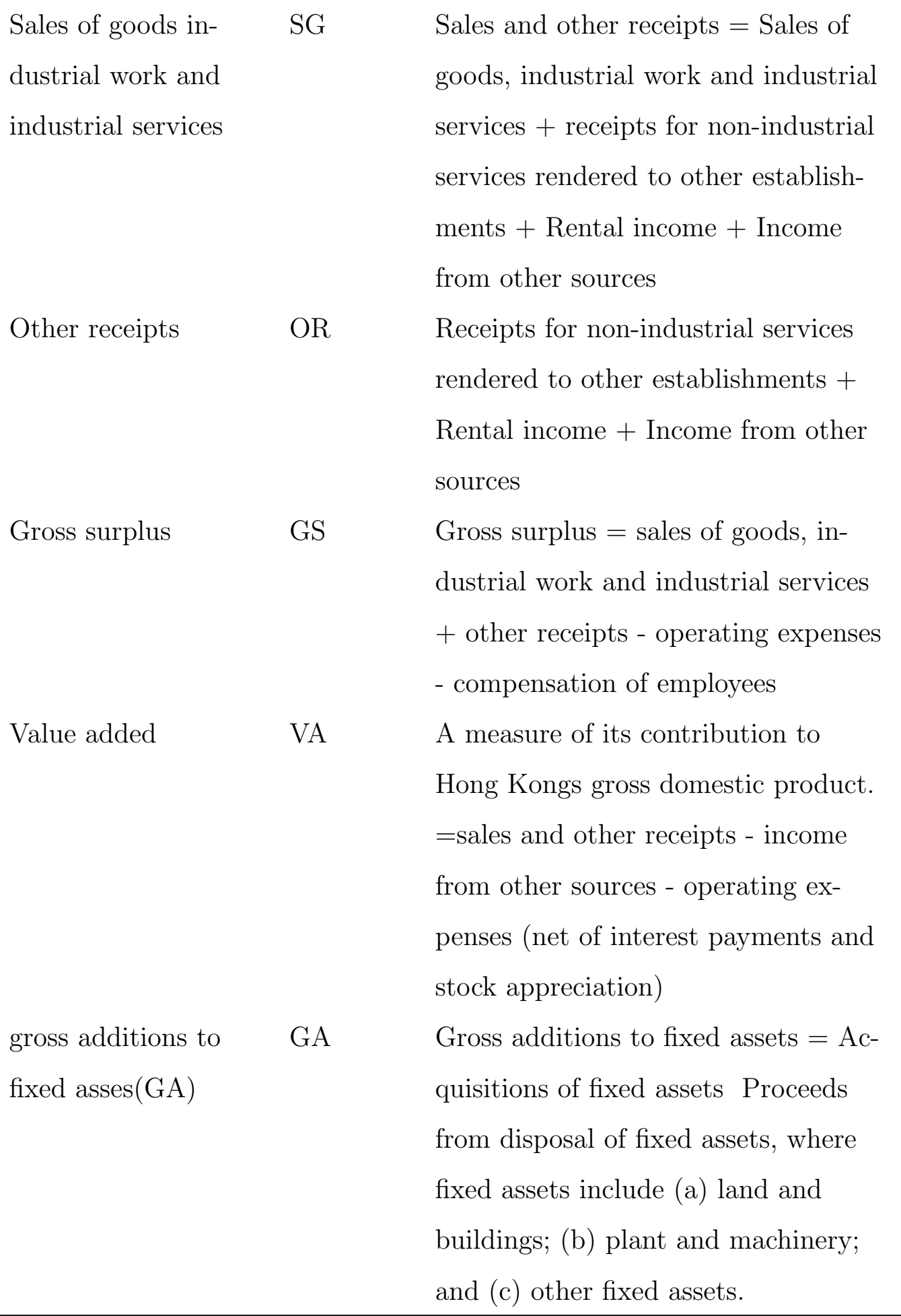

Construction sec- Number of projects NP Number of projects tor 


$\begin{array}{llll}\text { Census and } & \text { Total project ex- } & \text { TPE } & \text { Payments to contractors }+ \text { building } \\ \text { Statistics Depart- } & \text { penses } & & \text { materials and fittings supplied } \\ \text { ment } & & \text { outside the contract price }+ \text { ar- } \\ & \text { chitectural design and technical } \\ & \text { consultancy fees }+ \text { interest payments } \\ & \text { to finance the real estate develop- } \\ & \text { ment projects }+ \text { other project ex- } \\ & \text { penses }(=\mathrm{PC}+\mathrm{BMFS}+\mathrm{ADTCF}+\mathrm{IP}+\mathrm{OPE})\end{array}$

Payments to con- $\quad$ PC Payments to constructors structors

Building materials \& BMFS Building materials \& fittings supplied fittings supplied

Architectural de-

ADTCF Architectural design\& technical consign\& technical consultancy fees

sultancy fees

Interest payments IP

IP Interest payments

Other project ex- OPE Other project expenses penses

Value accrued to VCP project

P:market value of the property as if completed

L:market value of the underlying land lot

C:percentage of project completion 1: the beginning of reporting period 2:the end of reporting period Estimated land price ELPA Estimated land price appreciaappreciations tions(ELPA) 
Land area of

projects

Gross floor area of

buildings when com-

pleted
LAP Land area of projects(LAP)

GLABC

The gross floor area of a building is defined as the area contained within the external walls of the building measured at each floor level, including balconies and thickness of the external walls.

\begin{tabular}{|c|c|c|c|}
\hline $\begin{array}{l}\text { Populatio } n \text { and } \\
\text { household }\end{array}$ & $\begin{array}{l}\text { Average household } \\
\text { size }\end{array}$ & AHS & $\begin{array}{l}\text { Average number of household mem- } \\
\text { bers in a domestic household }\end{array}$ \\
\hline Census and & Percentage of house- & PHCWF & Percentage of households occupying a \\
\hline Statistics Depart- & holds occupying a & & whole flat \\
\hline ment & whole flat & & \\
\hline & Percentage of owner & POCG & Number of owner-occupier house- \\
\hline & occupier house- & & holds per 100 domestic households \\
\hline & $\operatorname{holds}(\%)$ & & \\
\hline & & & Owner-occupancy or home-ownership \\
\hline & & & is a form of housing tenure where a \\
\hline & & & person, called the owner-occupier, \\
\hline & & & owner-occupant, or home owner, \\
\hline & & & owns the home in which they live. \\
\hline & & & This home can be house, apartment, \\
\hline & & & condominium, or a housing coopera- \\
\hline & & & tive. In addition to providing hous- \\
\hline & & & ing, owner-occupancy also functions \\
\hline & & & as a real estate investment. \\
\hline
\end{tabular}




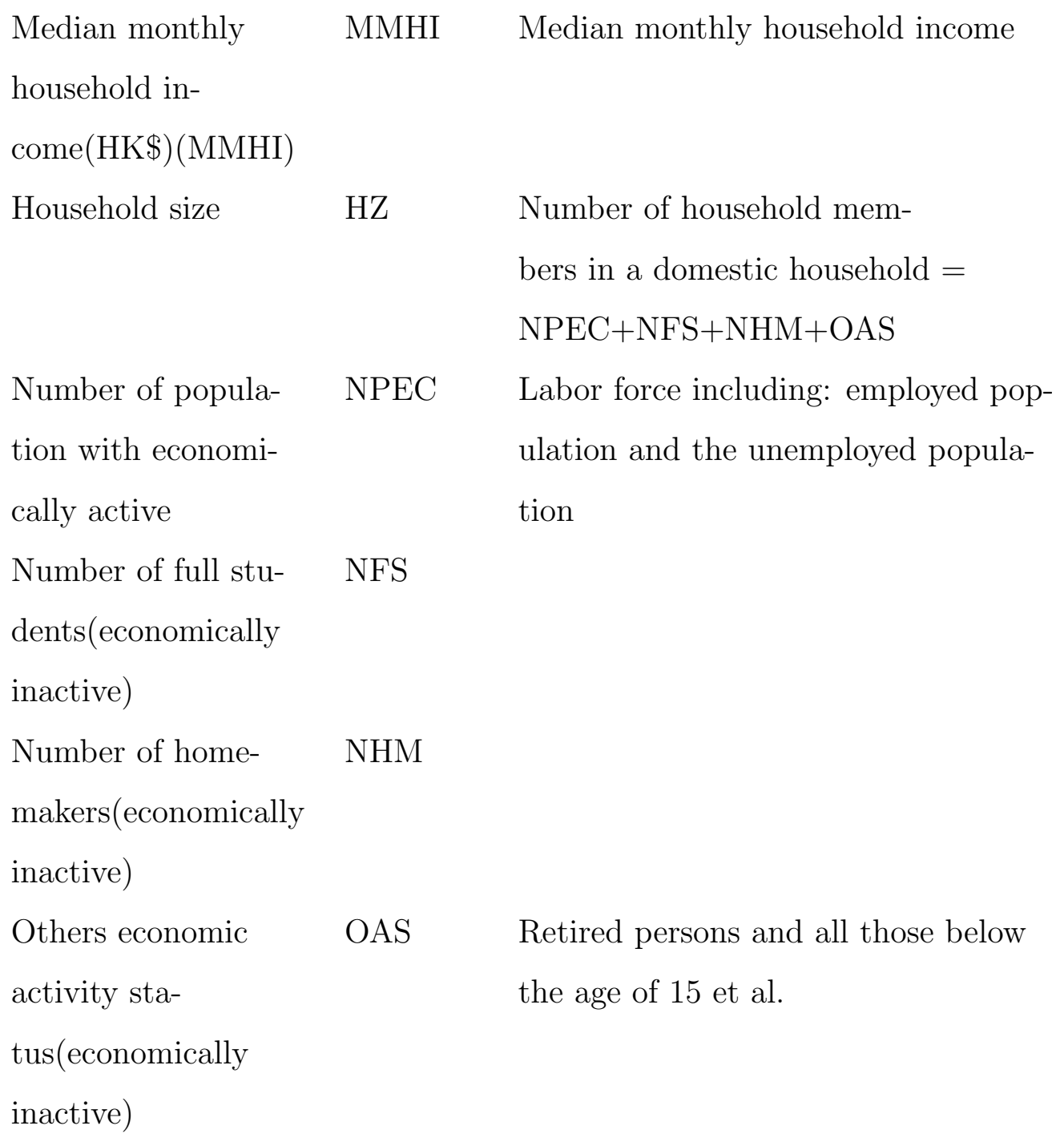

\begin{tabular}{|c|c|c|c|}
\hline $\begin{array}{l}\text { Traffic and trans- } \\
\text { portation }\end{array}$ & Traffic flow & $\mathrm{TF}$ & $\begin{array}{l}\text { Traffic flow at the street nearest to } \\
\text { air quality monitoring station }\end{array}$ \\
\hline Meteorology & wind direction & WD & $\begin{array}{l}\text { Hourly prevailing wind direction in } \\
\text { degree }\end{array}$ \\
\hline $\begin{array}{l}\text { Hong Kong Ob- } \\
\text { servatory }(\mathrm{HKO}\end{array}$ & $\begin{array}{l}\text { hourly mean wind } \\
\text { speed }\end{array}$ & WS & Hourly man wind speed in $\mathrm{m} / \mathrm{s}$ \\
\hline \multicolumn{4}{|l|}{ 2015) } \\
\hline & total cloud amount & CLD & (n okta) \\
\hline & $\begin{array}{l}\text { hourly maximum } \\
\text { gust (in } \mathrm{m} / \mathrm{s} \text { ) }\end{array}$ & GUST & $\begin{array}{l}\text { Annual average of hourly maximum } \\
\text { gust in } \mathrm{m} / \mathrm{s}\end{array}$ \\
\hline
\end{tabular}




\begin{tabular}{|c|c|c|c|}
\hline & $\begin{array}{l}\text { air temperature (in } \\
\text { degree Celsius) }\end{array}$ & TEMP & $\begin{array}{l}\text { Annual average of air temperature in } \\
\text { degree Celsius }\end{array}$ \\
\hline & $\begin{array}{l}\text { wet bulb tempera- } \\
\text { ture( in degree Cel- } \\
\text { sius) }\end{array}$ & WET & $\begin{array}{l}\text { Annual web bulb temperature in de- } \\
\text { gree Celsius }\end{array}$ \\
\hline & Dew point & DEW & (in degree Celsius) \\
\hline & Relative humidity & $\mathrm{RH}$ & $($ in $\%)$ \\
\hline & mean sea level pres- & MSLP & (in $\mathrm{hPa}$ ) \\
\hline & sure & & \\
\hline & $\begin{array}{l}\text { Average hourly rain- } \\
\text { fall }\end{array}$ & $\mathrm{RF}$ & (in $\mathrm{mm}$ ) \\
\hline Pollution & ozone & $\mathrm{O}_{3}$ & Annual average(in ppb) \\
\hline Environmental & Nitrogen dioxides & $\mathrm{NO}_{2}$ & Annual average(in ppb) \\
\hline Protection De- & & & \\
\hline partment(EPD) & & & \\
\hline of Hong & & & \\
\hline Kong(EPD,2014) & & & \\
\hline & Nitrogen oxides & $\mathrm{NO}_{x}$ & Annual average(in ppb) \\
\hline & Respirable sus- & $\mathrm{RSP} /$ & Annual average(in ppb) \\
\hline & pended particulates & $\mathrm{PM}_{10}$ & \\
\hline & sulphur dioxide & $\mathrm{SO}_{2}$ & Annual average(in ppb) \\
\hline
\end{tabular}

Table 1: Input and out variables 


\section{Bibliography}

[1] W. Faulkner, F. Badurdeen, Sustainable Value Stream Mapping (Sus-VSM): methodology to visualize and assess manufacturing sustainability performance, Journal of Cleaner Production 85 (2014) 8-18.

[2] EPA, Six Common Air Pollutants | Air \& Radiation | US EPA (2014).

[3] United States Environmental Protection Agency, What are the six common air pollutants? (Jun. 2015).

[4] S. H. M. Lam, S. M. Saunders, H. R. Cheng, H. Guo, Examination of regional ozone formation: POCPs for Western Australia and comparisons to other continents, Environmental Modelling \& Software 74 (2015) $194-200$.

[5] M. L. Bell, F. Dominici, J. M. Samet, A Meta-Analysis of Time-Series Studies of Ozone and Mortality With Comparison to the National Morbidity, Mortality, and Air Pollution Study, Epidemiology (Cambridge, Mass.) 16 (4) (2005) 436445.

[6] X. An, Q. Hou, N. Li, S. Zhai, Assessment of human exposure level to PM10 in China, Atmospheric Environment 70 (0) (2013) 376-386.

[7] M. Zhang, Y. Song, X. Cai, J. Zhou, Economic assessment of the health effects related to particulate matter pollution in 111 Chinese cities by using economic burden of disease analysis., Journal of environmental management 88 (4) (2008) 947-954.

[8] C. A. r. Pope, R. T. Burnett, M. J. Thun, E. E. Calle, D. Krewski, K. Ito, G. D. Thurston, Lung cancer, cardiopulmonary mortality, and long-term exposure to fine particulate air pollution., JAMA 287 (9) (2002) 1132-1141.

[9] X. Basagaa, B. Jacquemin, A. Karanasiou, B. Ostro, X. Querol, D. Agis, E. Alessandrini, J. Alguacil, B. Artiano, M. Catrambone, J. D. de la Rosa, J. Daz, A. Faustini, S. Ferrari, F. Forastiere, K. Katsouyanni, C. Linares, C. Perrino, A. Ranzi, I. Ricciardelli, E. Samoli, S. Zauli-Sajani, J. Sunyer, M. Stafoggia, Short-term effects of particulate matter constituents on daily hospitalizations and mortality in five South-European cities: Results from the MED-PARTICLES project, Environment International 75 (0) (2015) 151-158. 
[10] M. Pascal, G. Falq, V. Wagner, E. Chatignoux, M. Corso, M. Blanchard, S. Host, L. Pascal, S. Larrieu, Short-term impacts of particulate matter (PM10, PM102.5, PM2.5) on mortality in nine French cities, Atmospheric Environment 95 (0) (2014) 175-184.

[11] S. Araki, K. Iwahashi, H. Shimadera, K. Yamamoto, A. Kondo, Optimization of air monitoring networks using chemical transport model and search algorithm, Atmospheric Environment 122 (2015) 22 - 30.

[12] L. Wu, M. Bocquet, Optimal redistribution of the background ozone monitoring stations over France, Atmospheric Environment 45 (3) (2011) 772 - 783.

[13] A. Mofarrah, T. Husain, A holistic approach for optimal design of air quality monitoring network expansion in an urban area, Atmospheric Environment 44 (3) (2010) $432-440$.

[14] A. I. Khader, M. McKee, Use of a relevance vector machine for groundwater quality monitoring network design under uncertainty, Environmental Modelling \& Software 57 (2014) $115-126$.

[15] M. Fuentes, A. Chaudhuri, D. M. Holland, Bayesian entropy for spatial sampling design of environmental data, Environmental and Ecological Statistics 14 (3) (2007) 323-340.

[16] A. C. Comrie, Comparing Neural Networks and Regression Models for Ozone Forecasting, Journal of the Air \& Waste Management Association 47 (6) (1997) 653-663.

[17] S. Chattopadhyay, G. Bandyopadhyay, Artificial neural network with backpropagation learning to predict mean monthly total ozone in Arosa, Switzerland, International Journal of Remote Sensing 28 (20) (2007) 4471-4482.

[18] J. Jin, P. Ji, R. Gu, Identifying comparative customer requirements from product online reviews for competitor analysis, Engineering Applications of Artificial Intelligence 49 (2016) $61-73$.

[19] M. J. Shaw, C. Subramaniam, G. W. Tan, M. E. Welge, Knowledge management and data mining for marketing, Decision Support Systems 31 (1) (2001) 127 137, knowledge Management Support of Decision Making.

[20] I.-C. Yeh, C.-h. Lien, The comparisons of data mining techniques for the predictive accuracy of probability of default of credit card clients, Expert Systems with Applications 36 (2, Part 1) (2009) $2473-2480$.

[21] C.-L. Huang, M.-C. Chen, C.-J. Wang, Credit scoring with a data mining approach based on support vector machines, Expert Systems with Applications 33 (4) (2007) $847-856$. 
[22] H. C. Koh, G. Tan, et al., Data mining applications in healthcare, Journal of healthcare information management 19 (2) (2011) 65.

[23] M. Silver, T. Sakata, H.-C. Su, C. Herman, S. B. Dolins, M. J. O Shea, et al., Case study: how to apply data mining techniques in a healthcare data warehouse, Journal of healthcare information management 15 (2) (2001) 155-164.

[24] E. Rafalski, Using data mining/data repository methods to identify marketing opportunities in health care, Journal of Consumer Marketing 19 (7) (2002) 607-613.

[25] L. Hirschman, J. C. Park, J. Tsujii, L. Wong, C. H. Wu, Accomplishments and challenges in literature data mining for biology, Bioinformatics 18 (12) (2002) 1553-1561.

[26] W. Wang, W. Lu, X. Wang, A. Y. T. Leung, Prediction of maximum daily ozone level using combined neural network and statistical characteristics, Environment International 29 (5) (2003) $555-562$.

[27] S. M. Robeson, D. G. Steyn, Evaluation and comparison of statistical forecast models for daily maximum ozone concentrations, Atmospheric Environment. Part B, Urban Atmosphere, Volume 24, Issue 2, p. 303-312. 24 (1990) 303-312.

[28] G. M. McCollister, K. R. Wilson, Linear stochastic models for forecasting daily maxima and hourly concentrations of air pollutants, Atmospheric Environment (1967) 9 (4) (1975) $417-423$.

[29] P. Hájek, V. Olej, Ozone prediction on the basis of neural networks, support vector regression and methods with uncertainty, Ecological Informatics 12 (0) (2012) 31-42.

[30] E. Salazar-Ruiz, J. B. Ordieres, E. P. Vergara, S. F. Capuz-Rizo, Development and comparative analysis of tropospheric ozone prediction models using linear and artificial intelligence-based models in Mexicali, Baja California (Mexico) and Calexico, California (US), Environmental Modelling \& Software 23 (8) (2008) 1056 - 1069.

[31] W. Wang, C. Men, W. Lu, Online prediction model based on support vector machine, Neurocomputing 71 (46) (2008) 550 - 558.

[32] Y. Feng, W. Zhang, D. Sun, L. Zhang, Ozone concentration forecast method based on genetic algorithm optimized back propagation neural networks and support vector machine data classification, Atmospheric Environment 45 (11) (2011) 1979 - 1985.

[33] X. Yu, B. Yi, X. Wang, J. Chen, Predicting reaction rate constants of ozone with organic compounds from radical structures, Atmospheric Environment 51 (0) (2012) $124-130$. 
[34] U. Schlink, O. Herbarth, M. Richter, S. Dorling, G. Nunnari, G. Cawley, E. Pelikan, Statistical models to assess the health effects and to forecast ground-level ozone, Environmental Modelling \& Software 21 (4) (2006) 547 - 558.

[35] C.-h. Tsai, L.-c. Chang, H.-c. Chiang, Forecasting of ozone episode days by costsensitive neural network methods, Science of The Total Environment 407 (6) (2009) 2124-2135.

[36] K. Zhang, W. Fan, Forecasting skewed biased stochastic ozone days: analyses, solutions and beyond, Knowledge and Information Systems 14 (3) (2008) 299326.

[37] D. Birant, Comparison of decision tree algorithms for predicting potential air pollutant emissions with data mining models, Journal of Environmental Informatics 17 (1) (2011) 46-53.

[38] R. Chen, C. Chu, J. Tan, J. Cao, W. Song, X. Xu, C. Jiang, W. Ma, C. Yang, B. Chen, Y. Gui, H. Kan, Ambient air pollution and hospital admission in Shanghai, China, Journal of Hazardous Materials 181 (13) (2010) $234-240$.

[39] R. Chen, E. Samoli, C.-M. Wong, W. Huang, Z. Wang, B. Chen, H. Kan, Associations between short-term exposure to nitrogen dioxide and mortality in 17 Chinese cities: The China Air Pollution and Health Effects Study (CAPES), Environment International 45 (2012) $32-38$.

[40] M. Stafoggia, S. Zauli-Sajani, J. Pey, E. Samoli, E. Alessandrini, X. Basagaa, A. Cernigliaro, M. Chiusolo, M. Demaria, J. Daz, others, Desert Dust Outbreaks in Southern Europe: Contribution to Daily PM10 Concentrations and ShortTerm Associations with Mortality and Hospital Admissions., Environmental health perspectives.

[41] Y. Guo, Y. Jia, X. Pan, L. Liu, H.-E. Wichmann, The association between fine particulate air pollution and hospital emergency room visits for cardiovascular diseases in Beijing, China, Science of The Total Environment 407 (17) (2009) $4826-4830$.

[42] H.-Y. Liu, E. Skjetne, M. Kobernus, Mobile phone tracking: in support of modelling traffic-related air pollution contribution to individual exposure and its implications for public health impact assessment, Environmental Health 12 (1) (2013) 93.

[43] L. Fernandez-Luque, R. Karlsen, J. Bonander, Review of Extracting Information From the Social Web for Health Personalization, J Med Internet Res 13 (1) (2011) e15.

[44] A. Tumasjan, T. Sprenger, P. Sandner, I. Welpe, Predicting elections with twitter: What 140 characters reveal about political sentiment, in: Proceedings of the Fourth International AAAI Conference on Weblogs and Social Media, 2010, pp. 178-185. 
[45] H. Achrekar, A. Gandhe, R. Lazarus, S.-H. Yu, B. Liu, Predicting Flu Trends using Twitter data, in: Computer Communications Workshops (INFOCOM WKSHPS), 2011 IEEE Conference on, 2011, pp. 702-707.

[46] M. J. Paul, M. Dredze, Discovering Health Topics in Social Media Using Topic Models, PLoS ONE 9 (8) (2014) e103408.

[47] T. Sakaki, M. Okazaki, Y. Matsuo, Earthquake Shakes Twitter Users: Real-time Event Detection by Social Sensors, in: Proceedings of the 19th International Conference on World Wide Web, WWW '10, ACM, New York, NY, USA, 2010, pp. 851-860.

[48] D. Yates, S. Paquette, Emergency knowledge management and social media technologies: A case study of the 2010 Haitian earthquake, International Journal of Information Management 31 (1) (2011) 6-13.

[49] M. De Choudhury, Role of Social Media in Tackling Challenges in Mental Health, in: Proceedings of the 2Nd International Workshop on Socially-aware Multimedia, SAM '13, ACM, New York, NY, USA, 2013, pp. 49-52.

[50] W. Jiang, Y. Wang, M.-H. Tsou, X. Fu, Using Social Media to Detect Outdoor Air Pollution and Monitor Air Quality Index (AQI): A Geo-Targeted Spatiotemporal Analysis Framework with Sina Weibo (Chinese Twitter), PLoS ONE 10 (10) (2015) e0141185.

[51] M. Riga, M. Stocker, M. Rnkk, K. Karatzas, M. Kolehmainen, Atmospheric Environment and Quality of Life Information Extraction from Twitter with the Use of Self-Organizing Maps, JOURNAL OF ENVIRONMENTAL INFORMATICS 26 (1) (2015) 27-40.

[52] J. G. Olivier, J. A. Peters, G. Janssens-Maenhout, Trends in global co2 emissions 2015 report (2015).

[53] M. Garetti, M. Taisch, Sustainable manufacturing: trends and research challenges, Production Planning \& Control 23 (2-3) (2012) 83-104.

[54] M. Rudberg, M. Waldemarsson, H. Lidestam, Strategic perspectives on energy management: A case study in the process industry, Applied Energy 104 (2013) 487-496.

[55] N. Weinert, S. Chiotellis, G. Seliger, Methodology for planning and operating energy-efficient production systems, CIRP Annals-Manufacturing Technology 60 (1) (2011) 41-44.

[56] W. Wu, G. C. Dandy, H. R. Maier, Protocol for developing \{ann models and its application to the assessment of the quality of the $\{\mathrm{ann}\}$ model development process in drinking water quality modelling, Environmental Modelling \& Software 54 (0) (2014) 108 - 127. 
[57] R. Kohavi, G. H. John, Wrappers for feature subset selection, Artificial Intelligence 97 (12) (1997) $273-324$.

[58] Y. Zheng, X. Yi, M. Li, R. Li, Z. Shan, E. Chang, T. Li, Forecasting FineGrained Air Quality Based on Big Data, ACM Association for Computing Machinery, 2015.

[59] Y. Zheng, F. Liu, H.-P. Hsieh, U-Air: When Urban Air Quality Inference Meets Big Data, in: KDD 2013, ACM, 2013.

[60] A. L. P. Rojas, Simple atmospheric dispersion model to estimate hourly groundlevel nitrogen dioxide and ozone concentrations at urban scale, Environmental Modelling \& Software 59 (2014) 127 - 134.

[61] X.-H. Liu, Y. Zhang, S.-H. Cheng, J. Xing, Q. Zhang, D. G. Streets, C. Jang, W.-X. Wang, J.-M. Hao, Understanding of regional air pollution over China using CMAQ, part I performance evaluation and seasonal variation, Atmospheric Environment 44 (20) (2010) $2415-2426$.

[62] Y. Zhang, M. Bocquet, V. Mallet, C. Seigneur, A. Baklanov, Real-time air quality forecasting, part I: History, techniques, and current status, Atmospheric Environment 60 (2012) $632-655$.

[63] P. Yang, Y. Hwa Yang, B. B. Zhou, A. Y. Zomaya, A review of ensemble methods in bioinformatics, Current Bioinformatics 5 (4).

[64] K. P. Singh, S. Gupta, P. Rai, Identifying pollution sources and predicting urban air quality using ensemble learning methods, Atmospheric Environment 80 (0) (2013) $426-437$.

[65] K. Siwek, S. Osowski, Improving the accuracy of prediction of PM10 pollution by the wavelet transformation and an ensemble of neural predictors, Engineering Applications of Artificial Intelligence 25 (6) (2012) 1246-1258.

[66] E. Debry, V. Mallet, Ensemble forecasting with machine learning algorithms for ozone, nitrogen dioxide and PM10 on the Prev'Air platform, Atmospheric Environment 91 (2014) 71-84.

[67] C. Drummond, R. C. Holte, C4.5, Class Imbalance, and Cost Sensitivity: Why Under-sampling beats Over-sampling, 2003, pp. 1-8.

[68] N. V. Chawla, N. Japkowicz, A. Kotcz, Editorial: Special issue on learning from imbalanced data sets, SIGKDD Explor. Newsl. 6 (1) (2004) 1-6.

[69] Y. Sun, A. K. Wong, M. S. Kamel, Classification of imbalanced data: A review, International Journal of Pattern Recognition and Artificial Intelligence 23 (04) (2009) 687-719. 
[70] Y. Zhao, A. K. Shrivastava, K. L. Tsui, Imbalanced classification by learning hidden data structure, IIE Transactions 0 (0) (2014) 1-15.

[71] W.-Z. Lu, D. Wang, Ground-level ozone prediction by support vector machine approach with a cost-sensitive classification scheme, Science of The Total Environment 395 (23) (2008) 109-116.

[72] T. Fontes, L. M. Silva, M. P. Silva, N. Barros, A. C. Carvalho, Can artificial neural networks be used to predict the origin of ozone episodes?, Science of The Total Environment 488-489 (2014) $197-207$.

[73] V. López, A. Fernández, J. G. Moreno-Torres, F. Herrera, Analysis of preprocessing vs. cost-sensitive learning for imbalanced classification. Open problems on intrinsic data characteristics, Expert Systems with Applications 39 (7) (2012) 6585-6608.

[74] M. Galar, A. Fernandez, E. Barrenechea, H. Bustince, F. Herrera, A review on ensembles for the class imbalance problem: Bagging-, boosting-, and hybridbased approaches, Systems, Man, and Cybernetics, Part C: Applications and Reviews, IEEE Transactions on 42 (4) (2012) 463-484.

[75] L. Wu, M. Bocquet, M. Chevallier, Optimal Reduction of the Ozone Monitoring Network over France, Atmospheric environment (44).

[76] F. Shrouf, G. Miragliotta, Energy management based on Internet of Things: practices and framework for adoption in production management, Journal of Cleaner Production 100 (2015) 235-246.

[77] K. Vikhorev, R. Greenough, N. Brown, An advanced energy management framework to promote energy awareness, Journal of Cleaner Production 43 (2013) $103-112$.

[78] P. Maniatis, Investigating factors influencing consumer decision-making while choosing green products, Journal of Cleaner Production.

[79] Á. M. Ritter, M. Borchardt, G. L. Vaccaro, G. M. Pereira, F. Almeida, Motivations for promoting the consumption of green products in an emerging country: exploring attitudes of Brazilian consumers, Journal of Cleaner Production 106 (2015) 507-520.

[80] A. Vijayaraghavan, D. Dornfeld, Automated energy monitoring of machine tools, \{CIRP\} Annals - Manufacturing Technology 59 (1) (2010) 21 - 24.

[81] R. Kannan, W. Boie, Energy management practices in SME-case study of a bakery in Germany, Energy Conversion and Management 44 (6) (2003) 945 959. 
[82] M. Kurdve, M. Zackrisson, M. Wiktorsson, U. Harlin, Lean and green integration into production system models-experiences from swedish industry, Journal of Cleaner Production 85 (2014) 180-190.

[83] G. May, I. Barletta, B. Stahl, M. Taisch, Energy management in production: A novel method to develop key performance indicators for improving energy efficiency, Applied Energy 149 (2015) 46 - 61.

[84] V. Introna, V. Cesarotti, M. Benedetti, S. Biagiotti, R. Rotunno, Energy Management Maturity Model: an organizational tool to foster the continuous reduction of energy consumption in companies, Journal of Cleaner Production 83 (2014) 108-117.

[85] K. Bunse, M. Vodicka, P. Schönsleben, M. Brülhart, F. O. Ernst, Integrating energy efficiency performance in production management - gap analysis between industrial needs and scientific literature, Journal of Cleaner Production 19 (6-7) (2011) $667-679$.

[86] I. 50001, Energy management systems - Requirements with guidance for use, International Organization for Standardization (ISO)., Tech. rep. (2011).

[87] P. Ahi, C. Searcy, A comparative literature analysis of definitions for green and sustainable supply chain management, Journal of Cleaner Production 52 (2013) 329-341.

[88] S. Yu, Y.-M. Wei, J. Fan, X. Zhang, K. Wang, Exploring the regional characteristics of inter-provincial CO 2 emissions in China: an improved fuzzy clustering analysis based on particle swarm optimization, Applied energy 92 (2012) 552562.

[89] Y. Su, X. Chen, Y. Li, J. Liao, Y. Ye, H. Zhang, N. Huang, Y. Kuang, China?s 19-year city-level carbon emissions of energy consumptions, driving forces and regionalized mitigation guidelines, Renewable and Sustainable Energy Reviews 35 (2014) $231-243$.

[90] P. Tapio, D. Banister, J. Luukkanen, J. Vehmas, R. Willamo, Energy and transport in comparison: Immaterialisation, dematerialisation and decarbonisation in the $\{$ EU15\} between 1970 and 2000, Energy Policy 35 (1) (2007) 433 - 451.

[91] S. Yu, Y.-M. Wei, K. Wang, Provincial allocation of carbon emission reduction targets in China: An approach based on improved fuzzy cluster and Shapley value decomposition, Energy Policy 66 (2014) 630 - 644.

[92] P. Ekins, Economic growth and environmental sustainability: the prospects for green growth, Routledge, 2002.

[93] R. Khatibi, L. Naghipour, M. A. Ghorbani, M. S. Smith, V. Karimi, R. Farhoudi, H. Delafrouz, H. Arvanaghi, Developing a predictive tropospheric ozone model for tabriz, Atmospheric Environment 68 (0) (2013) 286-294. 
[94] C. Ambroise, Y. Grandvalet, Prediction of ozone peaks by mixture models, Ecological Modelling 145 (23) (2001) 275 - 289.

[95] J. Yi, V. R. Prybutok, A neural network model forecasting for prediction of daily maximum ozone concentration in an industrialized urban area, Environmental Pollution 92 (3) (1996) 349-357.

[96] J. Pires, A. Souza, H. Pavo, F. Martins, Variation of surface ozone in Campo Grande, Brazil: meteorological effect analysis and prediction, Environmental Science and Pollution Research 21 (17) (2014) 10550-10559.

[97] S. A. Abdul-Wahab, C. S. Bakheit, S. M. Al-Alawi, Principal component and multiple regression analysis in modelling of ground-level ozone and factors affecting its concentrations, Environmental Modelling \& Software 20 (10) (2005) $1263-1271$.

[98] J. Ruiz-Surez, O. Mayora-Ibarra, J. Torres-Jimnez, L. Ruiz-Surez, Short-term ozone forecasting by artificial neural networks, Advances in Engineering Software 23 (3) (1995) 143-149.

[99] N. Loya, I. Olmos Pineda, D. Pinto, H. Gmez-Adorno, Y. Alemn, Forecast of Air Quality Based on Ozone by Decision Trees and Neural Networks, in: I. Batyrshin, M. Gonzlez Mendoza (Eds.), Advances in Artificial Intelligence, Vol. 7629 of Lecture Notes in Computer Science, Springer Berlin Heidelberg, 2013, pp. 97-106.

[100] T. Wang, Y. Y. Wu, T. F. Cheung, K. S. Lam, A study of surface ozone and the relation to complex wind flow in hong kong, Atmospheric Environment 35 (18) (2001) $3203-3215$.

[101] EPD, Environmental protection interactive centre: Inquire and download air quality monitoring data (2014).

[102] H. K. O. HKO, Information of weather station (2014).

[103] Iberian Market Operator (2014).

[104] IDAE, Proposal for recognized document: CO2 emission factors and conversion factors from different sources of energy to energy being used in Spanish building sector (in Spanish), Tech. rep. (2014).

[105] A. Luna, M. Paredes, G. de Oliveira, S. Corra, Prediction of ozone concentration in tropospheric levels using artificial neural networks and support vector machine at Rio de Janeiro, Brazil, Atmospheric Environment 98 (0) (2014) 98-104.

[106] J. M. Rajab, M. Z. MatJafri, H. S. Lim, Combining multiple regression and principal component analysis for accurate predictions for column ozone in peninsular malaysia, Atmospheric Environment 71 (0) (2013) $36-43$. 
[107] S. M. Al-Alawi, S. A. Abdul-Wahab, C. S. Bakheit, Combining principal component regression and artificial neural networks for more accurate predictions of ground-level ozone, Environmental Modelling \& Software 23 (4) (2008) 396 -403 .

[108] S. I. V. Sousa, F. G. Martins, M. C. M. Alvim-Ferraz, M. C. Pereira, Multiple linear regression and artificial neural networks based on principal components to predict ozone concentrations, Environmental Modelling \& Software 22 (1) (2007) $97-103$.

[109] Y. Zhang, S. Wang, P. Phillips, G. Ji, Binary pso with mutation operator for feature selection using decision tree applied to spam detection, KnowledgeBased Systems 64 (2014) $22-31$.

[110] M. J. Brusco, A comparison of simulated annealing algorithms for variable selection in principal component analysis and discriminant analysis, Computational Statistics \& Data Analysis 77 (0) (2014) 38-53.

[111] I. Guyon, A. Elisseeff, An Introduction to Variable and Feature Selection, J. Mach. Learn. Res. 3 (2003) 1157-1182.

[112] Y. Saeys, I. Inza, P. Larraaga, A review of feature selection techniques in bioinformatics, Bioinformatics 23 (19) (2007) 2507-2517. arXiv:http://bioinformatics.oxfordjournals.org/content/23/19/ 2507.full.pdf+html.

[113] R. Meiri, J. Zahavi, Using simulated annealing to optimize the feature selection problem in marketing applications, European Journal of Operational Research 171 (3) (2006) 842 - 858, feature Cluster: Heuristic and Stochastic Methods in OptimizationFeature Cluster: New Opportunities for Operations Research.

[114] P. Bertolazzi, G. Felici, P. Festa, G. Fiscon, E. Weitschek, Integer programming models for feature selection: New extensions and a randomized solution algorithm, European Journal of Operational Research 250 (2) (2016) 389 - 399.

[115] S. Kirkpatrick, C. D. Gelatt, M. P. Vecchi, Optimization by simulated annealing, Science 220 (4598) (1983) 671-680. arXiv:http://www.sciencemag.org/ content/220/4598/671.full.pdf.

[116] M. Galar, A. Fernandez, E. Barrenechea, H. Bustince, F. Herrera, A review on ensembles for the class imbalance problem: Bagging-, boosting-, and hybridbased approaches, Systems, Man, and Cybernetics, Part C: Applications and Reviews, IEEE Transactions on 42 (4) (2012) 463-484.

[117] H. He, E. A. Garcia, Learning from Imbalanced Data, IEEE Transactions on Knowledge and Data Engineering 21 (9) (2009) 1263-1284. 
[118] N. V. Chawla, K. W. Bowyer, L. O. Hall, W. P. Kegelmeyer, Smote: Synthetic minority over-sampling technique, Journal of Artificial Intelligence Research 16 (2002) 321-357.

[119] R. Akbani, S. Kwek, N. Japkowicz, Applying support vector machines to imbalanced datasets, in: Machine learning: ECML 2004, Springer, 2004, pp. 39-50.

[120] H. Hettige, P. Martin, M. Singh, D. Wheeler, The industrial pollution projection system Policy, Research working paper (1431).

[121] T. H. Oiamo, M. Johnson, K. Tang, I. N. Luginaah, Assessing traffic and industrial contributions to ambient nitrogen dioxide and volatile organic compounds in a low pollution urban environment, Science of The Total Environment 529 (2015) $149-157$.

[122] C. Y. Chan, L. Y. Chan, X. M. Wang, Y. M. Liu, S. C. Lee, S. C. Zou, G. Y. Sheng, J. M. Fu, Volatile organic compounds in roadside microenvironments of metropolitan Hong Kong, Atmospheric Environment 36 (12) (2002) 2039 2047.

[123] T. Slini, A. Kaprara, K. Karatzas, N. Moussiopoulos, \{PM10\} forecasting for thessaloniki, greece, Environmental Modelling \& Software 21 (4) (2006) 559 565.

[124] Ca, rlos G, ersh, ens, Ca, rlos G, ersh, ens, Carlos Gershenson, Artificial neural networks for beginners.

[125] W. McCulloch, W. Pitts, A logical calculus of the ideas immanent in nervous activity, The bulletin of mathematical biophysics 5 (4) (1943) 115-133.

[126] J. Ordieres, E. Vergara, R. Capuz, R. Salazar, Neural network prediction model for fine particulate matter (PM2.5) on the US-Mexico border in El Paso (Texas) and Ciudad Juárez (Chihuahua), Environmental Modelling \& Software 20 (5) (2005) 547-559.

[127] L. Breiman, Classification and regression trees, Wadsworth International Group, Belmont, Calif, 1984.

[128] D. Gacquer, V. Delcroix, F. Delmotte, S. Piechowiak, Comparative study of supervised classification algorithms for the detection of atmospheric pollution, Engineering Applications of Artificial Intelligence 24 (6) (2011) 1070-1083.

[129] S. Osowski, K. Garanty, Forecasting of the daily meteorological pollution using wavelets and support vector machine, Engineering Applications of Artificial Intelligence 20 (6) (2007) $745-755$.

[130] B. Evgeny, F. Uli, S. Jens, S. Gisbert, Comparison of Support Vector Machine and Artificial Neural Network Systems for Drug/Nondrug Classification, Journal of Chemical Information and Computer Sciences 43 (6) (2003) 1882-1889. 
[131] J. Li, An empirical comparison between SVMs and ANNs for speech recognition, in: The First Instructional Conf. on Machine Learning, Vol. 951, 2003, p. 2003.

[132] L. Breiman, Random forests, Machine Learning 45 (1) (2001) 532.

[133] R. Caruana, A. Niculescu-Mizil, G. Crew, A. Ksikes, Ensemble selection from libraries of models, in: Proceedings of the Twenty-first International Conference on Machine Learning, ICML '04, ACM, New York, NY, USA, 2004, p. 18.

[134] M. H. Zhang, Q. S. Xu, F. Daeyaert, P. J. Lewi, D. L. Massart, Application of boosting to classification problems in chemometrics, Analytica Chimica Acta 544 (12) (2005) 167 - 176, papers Presented at the 9th International Conference on Chemometrics in Analytical ChemistryPapers Presented at the 9th International Conference on Chemometrics in Analytical chemistry.

[135] D.-S. Cao, Q.-S. Xu, Y.-Z. Liang, L.-X. Zhang, H.-D. Li, The boosting: A new idea of building models, Chemometrics and Intelligent Laboratory Systems 100 (1) (2010) $1-11$.

[136] R. A. Roy, J. P. Nair, E. Sherly, Decision tree based data classification for marine wireless communication, in: 2015 International Conference on Computing and Network Communications (CoCoNet), 2015, pp. 633-638.

[137] C.-F. Tsai, Y.-F. Hsu, D. C. Yen, A comparative study of classifier ensembles for bankruptcy prediction, Applied Soft Computing 24 (2014) 977 - 984.

[138] L. G. Valiant, A Theory of the Learnable, Commun. ACM 27 (11) (1984) 1134-1142.

[139] M. Kearns, L. Valiant, Cryptographic Limitations on Learning Boolean Formulae and Finite Automata, J. ACM 41 (1) (1994) 67-95.

[140] Y. Freund, R. E. Schapire, A Decision-Theoretic Generalization of On-Line Learning and an Application to Boosting, J. Comput. Syst. Sci. 55 (1) (1997) 119-139.

[141] J. Zheng, Cost-sensitive boosting neural networks for software defect prediction, Expert Systems with Applications 37 (6) (2010) 4537 - 4543.

[142] R. E. Schapire, A Brief Introduction to Boosting, in: Proceedings of the 16th International Joint Conference on Artificial Intelligence - Volume 2, IJCAI'99, Morgan Kaufmann Publishers Inc., San Francisco, CA, USA, 1999, pp. 14011406.

[143] G. Wang, J. Hao, J. Ma, H. Jiang, A comparative assessment of ensemble learning for credit scoring, Expert Systems with Applications 38 (1) (2011) 223 -230 .

[144] G. Ridgeway, Generalized boosted models: A guide to the gbm package. 
[145] L. Breiman, Stacked Regressions, Machine Learning 24 (1) (1996) 49-64.

[146] S. Ali, K. A. Smith, On learning algorithm selection for classification, Applied Soft Computing 6 (2) (2006) 119-138.

[147] Q. Gu, L. Zhu, Z. Cai, Evaluation measures of the classification performance of imbalanced data sets, in: Z. Cai, Z. Li, Z. Kang, Y. Liu (Eds.), Computational Intelligence and Intelligent Systems, Vol. 51 of Communications in Computer and Information Science, Springer Berlin Heidelberg, 2009, pp. 461-471.

[148] N. D. Bennett, B. F. W. Croke, G. Guariso, J. H. A. Guillaume, S. H. Hamilton, A. J. Jakeman, S. Marsili-Libelli, L. T. H. Newham, J. P. Norton, C. Perrin, S. A. Pierce, B. Robson, R. Seppelt, A. A. Voinov, B. D. Fath, V. Andreassian, Characterising performance of environmental models, Environmental Modelling \& Software 40 (0) (2013) $1-20$.

[149] A. J. Torija, D. P. Ruiz, A general procedure to generate models for urban environmental-noise pollution using feature selection and machine learning methods, Science of The Total Environment 505 (2015) 680 - 693.

[150] G. Haixiang, L. Yijing, L. Yanan, L. Xiao, L. Jinling, BPSO-Adaboost-KNN ensemble learning algorithm for multi-class imbalanced data classification, Engineering Applications of Artificial Intelligence 49 (2016) 176 - 193.

[151] P. D. Bridge, S. S. Sawilowsky, Increasing Physicians Awareness of the Impact of Statistics on Research Outcomes: Comparative Power of the t-test and Wilcoxon Rank-Sum Test in Small Samples Applied Research, Journal of Clinical Epidemiology 52 (3) (1999) 229 - 235.

[152] IPCC, Intergovernmental Panel on Climate Change (2006).

[153] R. Mitchell, Web scraping with Python: collecting data from the modern web, " O’Reilly Media, Inc.", 2015.

[154] R. Ruiz-Cárdenas, M. A. Ferreira, A. M. Schmidt, Stochastic search algorithms for optimal design of monitoring networks, Environmetrics 21 (1) (2010) 102112.

[155] N. D. Le, J. V. Zidek, Statistical analysis of environmental space-time processes, Springer Science \& Business Media, 2006.

[156] W. G. Müller, Collecting spatial data: optimum design of experiments for random fields, Springer Science \& Business Media, 2007.

[157] O. road particulate matter (PM2.5), H. K. gaseous emissions in the Shing Mun Tunnel, S. C. Lee, Y. Gao, L. Cui, W. Deng, J. Cao, Z. Shen, J. Sun, Real-time measurements of PM2.5, PM102.5, and $\{\mathrm{BC}\}$ in an urban street canyon, Particuology 20 (0) (2015) 134 - 140, origin, evolution, and distribution of atmospheric aerosol particles in Asia. 
[158] Y. Cheng, S. C. Lee, K. F. Ho, P. K. K. Louie, On-road particulate matter (PM2.5) and gaseous emissions in the Shing Mun Tunnel, Hong Kong, Atmospheric Environment 40 (23) (2006) 4235 - 4245.

[159] P. H. Ryan, G. K. LeMasters, A Review of Land-use Regression Models for Characterizing Intraurban Air Pollution Exposure, Inhalation toxicology 19 (Suppl 1) (2007) 127-133.

[160] M. Brauer, G. Hoek, P. v. Vliet, K. Meliefste, P. Fischer, U. Gehring, J. Heinrich, J. Cyrys, T. Bellander, M. Lewne, B. Brunekreef, Estimating Long-Term Average Particulate Air Pollution Concentrations: Application of Traffic Indicators and Geographic Information Systems, Epidemiology 14 (2) (2003) $228-239$.

[161] J. Zhang, K. R. Smith, Y. Ma, S. Ye, F. Jiang, W. Qi, P. Liu, M. A. K. Khalil, R. A. Rasmussen, S. A. Thorneloe, Greenhouse gases and other airborne pollutants from household stoves in China: a database for emission factors, Atmospheric Environment 34 (26) (2000) 4537 - 4549.

[162] J. Meng, J. Liu, Y. Xu, S. Tao, Tracing Primary PM 2.5 emissions via Chinese supply chains, Environmental Research Letters 10 (5) (2015) 054005.

[163] D. Guan, X. Su, Q. Zhang, G. P. Peters, Z. Liu, Y. Lei, K. He, The socioeconomic drivers of Chinas primary PM 2.5 emissions, Environmental Research Letters 9 (2) (2014) 024010.

[164] H. Zhang, D. Zhai, Y. N. Yang, Simulation-based estimation of environmental pollutions from construction processes, Journal of Cleaner Production 76 (2014) $85-94$.

[165] G. Qi, L. Shen, S. Zeng, O. J. Jorge, The drivers for contractors green innovation: an industry perspective, Journal of Cleaner Production 18 (14) (2010) 1358-1365.

[166] Y. H. Dong, S. T. Ng, A life cycle assessment model for evaluating the environmental impacts of building construction in Hong Kong, Building and Environment 89 (2015) $183-191$.

[167] K. W. Appel, R. C. Gilliam, N. Davis, A. Zubrow, S. C. Howard, Overview of the atmospheric model evaluation tool (AMET) v1.1 for evaluating meteorological and air quality models, Environmental Modelling \& Software 26 (4) (2011) 434 $-443$.

[168] H.J.S. Fernando, Forecasting PM10 in metropolitan areas: Efficacy of neural networks, Environmental pollution 163 (2012) (2011) 62-67.

[169] K. Chrysostomou, S. Y. Chen, X. Liu, Combining multiple classifiers for wrapper feature selection, International Journal of Data Mining, Modelling and Management 1 (1) (2008) 91-102. 
[170] D. K. Chandra, V. Ravi, P. Ravisankar, Support vector machine and wavelet neural network hybrid: application to bankruptcy prediction in banks, International Journal of Data Mining, Modelling and Management 2 (1) (2010) $1-21$.

[171] C.-F. Tsai, Feature selection in bankruptcy prediction, Knowledge-Based Systems 22 (2) (2009) $120-127$.

[172] L. Rokach, Genetic algorithm-based feature set partitioning for classification problems, Pattern Recognition 41 (5) (2008) 1676 - 1700.

[173] H. R. Maier, A. Jain, G. C. Dandy, K. P. Sudheer, Methods used for the development of neural networks for the prediction of water resource variables in river systems: Current status and future directions, Environmental Modelling \& Software 25 (8) (2010) $891-909$.

[174] J. C. M. Pires, F. G. Martins, Correction methods for statistical models in tropospheric ozone forecasting, Atmospheric Environment 45 (14) (2011) 2413 $-2417$.

[175] B. Özbay, G. A. Keskin, Ş. Ç. Doğruparmak, S. Ayberk, Predicting tropospheric ozone concentrations in different temporal scales by using multilayer perceptron models, Ecological Informatics 6 (34) (2011) $242-247$.

[176] M. L. Thompson, J. Reynolds, L. H. Cox, P. Guttorp, P. D. Sampson, A review of statistical methods for the meteorological adjustment of tropospheric ozone, Atmospheric Environment 35 (3) (2001) 617 - 630.

[177] H. Çamdevýren, N. Demýr, A. Kanik, S. Keskýn, Use of principal component scores in multiple linear regression models for prediction of Chlorophyll-a in reservoirs, Ecological Modelling 181 (4) (2005) 581 - 589.

[178] J. C. M. Pires, F. G. Martins, S. I. V. Sousa, M. C. M. Alvim-Ferraz, M. C. Pereira, Selection and validation of parameters in multiple linear and principal component regressions, Environmental Modelling \& Software 23 (1) (2008) 50 -55 .

[179] V. R. Prybutok, J. Yi, D. Mitchell, Comparison of neural network models with \{arima\} and regression models for prediction of houston's daily maximum ozone concentrations, European Journal of Operational Research 122 (1) (2000) 31 40 .

[180] L. A. Díaz-Robles, J. C. Ortega, J. S. Fu, G. D. Reed, J. C. Chow, J. G. Watson, J. A. Moncada-Herrera, A hybrid \{ARIMA\} and artificial neural networks model to forecast particulate matter in urban areas: The case of Temuco, Chile, Atmospheric Environment 42 (35) (2008) 8331 - 8340. 
[181] P. Goyal, A. T. Chan, N. Jaiswal, Statistical models for the prediction of respirable suspended particulate matter in urban cities, Atmospheric Environment 40 (11) (2006) $2068-2077$.

[182] G. Ibarra-Berastegi, A. Elias, A. Barona, J. Saenz, A. Ezcurra, J. D. d. Argandoa, From diagnosis to prognosis for forecasting air pollution using neural networks: Air pollution monitoring in Bilbao, Environmental Modelling \& Software 23 (5) (2008) $622-637$.

[183] S. A. Abdul-Wahab, S. M. Al-Alawi, Assessment and prediction of tropospheric ozone concentration levels using artificial neural networks, Environmental Modelling \& Software 17 (3) (2002) $219-228$.

[184] S. I. V. Sousa, F. G. Martins, M. C. Pereira, M. C. M. Alvim-Ferraz, Prediction of ozone concentrations in oporto city with statistical approaches, Chemosphere 64 (7) (2006) $1141-1149$.

[185] E. Agirre-Basurko, G. Ibarra-Berastegi, I. Madariaga, Regression and multilayer perceptron-based models to forecast hourly O3 and NO2 levels in the Bilbao area, Urban Air Quality Modelling Urban Air Quality Modelling 21 (4) (2006) 430-446.

[186] H.-C. Lu, J.-C. Hsieh, T.-S. Chang, Prediction of daily maximum ozone concentrations from meteorological conditions using a two-stage neural network, Atmospheric Research 81 (2) (2006) 124 - 139.

[187] B. Scholkopf, A. J. Smola, Learning with Kernels: Support Vector Machines, Regularization, Optimization, and Beyond, MIT Press, Cambridge, MA, USA, 2001.

[188] P. Hjek, V. Olej, Ozone prediction on the basis of neural networks, support vector regression and methods with uncertainty, Ecological Informatics 12 (0) (2012) 31-42.

[189] W.-Z. Lu, W.-J. Wang, Potential assessment of the support vector machine method in forecasting ambient air pollutant trends, Chemosphere 59 (5) (2005) $693-701$.

[190] F. Luan, C. Xue, R. Zhang, C. Zhao, M. Liu, Z. Hu, B. Fan, Prediction of retention time of a variety of volatile organic compounds based on the heuristic method and support vector machine, Analytica Chimica Acta 537 (12) (2005) $101-110$.

[191] C. Cortes, V. Vapnik, Support-vector networks, Machine Learning 20 (3) (1995) 273-297.

[192] M. Arhami, N. Kamali, M. Rajabi, Predicting hourly air pollutant levels using artificial neural networks coupled with uncertainty analysis by monte carlo simulations, Environmental Science and Pollution Research (2013) 1-13. 
[193] E. G. Ortiz-Garca, S. Salcedo-Sanz, . M. Prez-Bellido, J. A. Portilla-Figueras, L. Prieto, Prediction of hourly $\{03\}$ concentrations using support vector regression algorithms, Atmospheric Environment 44 (35) (2010) 4481 - 4488.

[194] S. Pöyhönen, A. Arkkio, P. Jover, H. Hyötyniemi, Coupling pairwise support vector machines for fault classification, Control Engineering Practice 13 (6) (2005) 759-769.

[195] P. J. G. Nieto, E. F. Combarro, J. J. d. C. Díaz, E. Montañés, A SVM-based regression model to study the air quality at local scale in oviedo urban area (northern spain): A case study, Applied Mathematics and Computation 219 (17) (2013) $8923-8937$.

[196] H. Sug, Ozone day prediction with radial basis function networks, in: Proceedings of the 14th WSEAS International Conference on Systems: Part of the 14th WSEAS CSCC Multiconference - Volume II, ICS'10, World Scientific and Engineering Academy and Society (WSEAS), Stevens Point, Wisconsin, USA, 2010, p. 608611 .

[197] K. Zhang, W. Fan, X. Yuan, I. Davidson, X. Li, W.: Forecasting skewed biased stochastic ozone days: analyses, solutions and beyond, Knowl. Inf. Syst.

[198] E. Solazzo, R. Bianconi, R. Vautard, K. W. Appel, M. D. Moran, C. Hogrefe, B. Bessagnet, J. Brandt, J. H. Christensen, C. Chemel, I. Coll, H. D. v. d. Gon, J. Ferreira, R. Forkel, X. V. Francis, G. Grell, P. Grossi, A. B. Hansen, A. Jerievi, L. Kraljevi, A. I. Miranda, U. Nopmongcol, G. Pirovano, M. Prank, A. Riccio, K. N. Sartelet, M. Schaap, J. D. Silver, R. S. Sokhi, J. Vira, J. Werhahn, R. Wolke, G. Yarwood, J. Zhang, S. T. Rao, S. Galmarini, Model evaluation and ensemble modelling of surface-level ozone in europe and north america in the context of \{aqmeii\}, Atmospheric Environment 53 (0) (2012) 60 - 74, $<$ ce:title>AQMEII: An International Initiative for the Evaluation of RegionalScale Air Quality Models - Phase $1</$ ce:title $>$.

[199] L. D. Monache, R. B. Stull, An ensemble air-quality forecast over western europe during an ozone episode, Atmospheric Environment 37 (25) (2003) 3469 - 3474.

[200] S. McKeen, J. Wilczak, G. Grell, I. Djalalova, S. Peckham, E.-Y. Hsie, W. Gong, V. Bouchet, S. Menard, R. Moffet, J. McHenry, J. McQueen, Y. Tang, G. R. Carmichael, M. Pagowski, A. Chan, T. Dye, G. Frost, P. Lee, R. Mathur, Assessment of an ensemble of seven real-time ozone forecasts over eastern north america during the summer of 2004, Journal of Geophysical Research: Atmospheres 110 (D21) (2005) n/an/a.

[201] I. Partalas, G. Tsoumakas, E. V. Hatzikos, I. Vlahavas, Greedy regression ensemble selection: Theory and an application to water quality prediction, Information Sciences 178 (20) (2008) 3867 - 3879. 
[202] D. Hasenfratz, O. Saukh, C. Walser, C. Hueglin, M. Fierz, T. Arn, J. Beutel, L. Thiele, Deriving high-resolution urban air pollution maps using mobile sensor nodes, Pervasive and Mobile Computing 16 (2015) 268-285.

[203] D. Hasenfratz, O. Saukh, S. Sturzenegger, L. Thiele, Participatory air pollution monitoring using smartphones, Mobile Sensing (2012) 1-5.

[204] S. Steinle, S. Reis, C. E. Sabel, Quantifying human exposure to air pollution-Moving from static monitoring to spatio-temporally resolved personal exposure assessment, Science of the Total Environment 443 (2013) 184-193.

[205] M. L. Glasgow, C. B. Rudra, E.-H. Yoo, M. Demirbas, J. Merriman, P. Nayak, C. Crabtree-Ide, A. A. Szpiro, A. Rudra, J. Wactawski-Wende, others, Using smartphones to collect time-activity data for long-term personal-level air pollution exposure assessment, Journal of Exposure Science and Environmental Epidemiology.

[206] Y. U. I. HUANG, S. Batterman, Residence location as a measure of environmental exposure: a review of air pollution epidemiology studies., Journal of Exposure Analysis \& Environmental Epidemiology 10 (1).

[207] N. D. Lane, E. Miluzzo, H. Lu, D. Peebles, T. Choudhury, A. T. Campbell, A survey of mobile phone sensing, IEEE Communications magazine 48 (9) (2010) $140-150$.

[208] J. G. Su, M. Jerrett, Y.-Y. Meng, M. Pickett, B. Ritz, Integrating smart-phone based momentary location tracking with fixed site air quality monitoring for personal exposure assessment, Science of The Total Environment 506 (2015) $518-526$.

[209] L. D. Mercer, A. A. Szpiro, L. Sheppard, J. Lindström, S. D. Adar, R. W. Allen, E. L. Avol, A. P. Oron, T. Larson, L.-J. S. Liu, et al., Comparing universal kriging and land-use regression for predicting concentrations of gaseous oxides of nitrogen (no x) for the multi-ethnic study of atherosclerosis and air pollution (mesa air), Atmospheric Environment 45 (26) (2011) 4412-4420.

[210] J. G. Su, M. Jerrett, B. Beckerman, D. Verma, M. A. Arain, P. Kanaroglou, D. Stieb, M. Finkelstein, J. Brook, A land use regression model for predicting ambient volatile organic compound concentrations in toronto, canada, Atmospheric Environment 44 (29) (2010) 3529-3537.

[211] A. d. Nazelle, S. Arunachalam, M. L. Serre, Bayesian maximum entropy integration of ozone observations and model predictions: an application for attainment demonstration in north carolina, Environmental science \& technology 44 (15) (2010) 5707-5713.

[212] A. De Nazelle, M. L. Serre, Ozone exposure assessment in north carolina using bayesian maximum entropy data integration of space time observations and air quality model prediction, Epidemiology 17 (6) (2006) S189. 
[213] S. D. Beevers, N. Kitwiroon, M. L. Williams, D. C. Carslaw, One way coupling of cmaq and a road source dispersion model for fine scale air pollution predictions, Atmospheric environment 59 (2012) 47-58.

[214] T. Maté, R. Guaita, M. Pichiule, C. Linares, J. Díaz, Short-term effect of fine particulate matter (PM2.5) on daily mortality due to diseases of the circulatory system in Madrid (Spain), Science of The Total Environment 408 (23) (2010) 5750 - 5757, special Section: Integrating Water and Agricultural Management Under Climate Change.

[215] N. A. H. Janssen, P. Fischer, M. Marra, C. Ameling, F. R. Cassee, Short-term effects of PM2.5, \{PM10\} and PM2.5-10 on daily mortality in the Netherlands, Science of The Total Environment 463-464 (2013) $20-26$.

[216] P. Garrett, E. Casimiro, Short-term effect of fine particulate matter (PM2.5) and ozone on daily mortality in Lisbon, Portugal, Environmental Science and Pollution Research 18 (9) (2011) 1585-1592.

[217] J. I. Halonen, T. Lanki, T. Yli-Tuomi, P. Tiittanen, M. Kulmala, J. Pekkanen, Particulate air pollution and acute cardiorespiratory hospital admissions and mortality among the elderly, Epidemiology 20 (1) (2009) 143-153.

[218] S. Wang, M. J. Paul, M. Dredze, Social Media as a Sensor of Air Quality and Public Response in China, Journal of Medical Internet Research 17 (3) (2015) $\mathrm{e} 22$.

[219] C. Herrmann, S. Thiede, Process chain simulation to foster energy efficiency in manufacturing, CIRP journal of manufacturing science and technology 1 (4) (2009) 221-229.

[220] Y. Seow, S. Rahimifard, A framework for modelling energy consumption within manufacturing systems, CIRP Journal of Manufacturing Science and Technology 4 (3) (2011) 258-264.

[221] S. Thiede, G. Bogdanski, C. Herrmann, A systematic method for increasing the energy and resource efficiency in manufacturing companies, Procedia CIRP 2 (2012) 28-33.

[222] A. Brown, J. Amundson, F. Badurdeen, Sustainable value stream mapping (Sus-VSM) in different manufacturing system configurations: application case studies, Journal of Cleaner Production 85 (2014) 164-179.

[223] H. Makita, Y. Shida, N. Nozue, Factory energy management system using production information, Mitsubishi Electric Advance 140 (2012) 7-11.

[224] S. Haller, S. Karnouskos, C. Schroth, The internet of things in an enterprise context, in: Future Internet Symposium, Springer, 2009, pp. 14-28. 
[225] G. Miragliotta, F. Shrouf, Using Internet of Things to improve eco-efficiency in manufacturing: a review on available knowledge and a framework for IoT adoption, in: IFIP International Conference on Advances in Production Management Systems, Springer, 2013, pp. 96-102.

[226] H. Sequeira, P. Carreira, T. Goldschmidt, P. Vorst, Energy Cloud: Real-Time Cloud-Native Energy Management System to Monitor and Analyze Energy Consumption in Multiple Industrial Sites, in: Utility and Cloud Computing (UCC), 2014 IEEE/ACM 7th International Conference on, 2014, pp. 529-534.

[227] C. Herrmann, C. Schmidt, D. Kurle, S. Blume, S. Thiede, Sustainability in Manufacturing and Factories of the Future, International Journal of Precision Engineering and Manufacturing-Green Technology 1 (4) (2014) 283-292.

[228] F. Shrouf, J. Ordieres, G. Miragliotta, Smart factories in industry 4.0: a review of the concept and of energy management approached in production based on the Internet of things paradigm, in: 2014 IEEE International Conference on Industrial Engineering and Engineering Management, IEEE, 2014, pp. 697-701.

[229] E. Zampou, S. Plitsos, A. Karagiannaki, I. Mourtos, Towards a framework for energy-aware information systems in manufacturing, Computers in Industry 65 (3) (2014) 419 - 433, \{ICT $\}$ for Sustainability in Industry.

[230] B. Gong, J. Ordieres-Meré, Prediction of daily maximum ozone threshold exceedances by preprocessing and ensemble artificial intelligence techniques: Case study of Hong Kong, Environmental Modelling \& Software 84 (2016) 290 - 303.

[231] A. Stojić, D. Maletić, S. S. Stojić, Z. Mijić, A. Šoštarić, Forecasting of VOC emissions from traffic and industry using classification and regression multivariate methods, Science of The Total Environment 521-522 (2015) $19-26$.

[232] F. Shrouf, B. Gong, J. Ordieres-Meré, Multi-level awareness of energy used in production processes, Journal of Cleaner Production.

[233] J. Davis, T. Edgar, J. Porter, J. Bernaden, M. Sarli, Smart manufacturing, manufacturing intelligence and demand-dynamic performance, Computers \& Chemical Engineering 47 (2012) 145-156.

[234] Iberdrola (2015).

[235] Gesternova (2015).

[236] N. Marz, J. Warren, Big Data: Principles and best practices of scalable realtime data systems, Manning Publications Co., 2015.

[237] T. O. Mahony, Decomposition of Ireland's carbon emissions from 1990 to 2010: An extended Kaya identity, Energy Policy 59 (2013) $573-581$. 
[238] W. Zhou, B. Zhu, D. Chen, C. Griffy-Brown, Y. Ma, W. Fei, Energy consumption patterns in the process of chinas urbanization, Population and Environment $33(2-3)(2012)$ 202-220. 